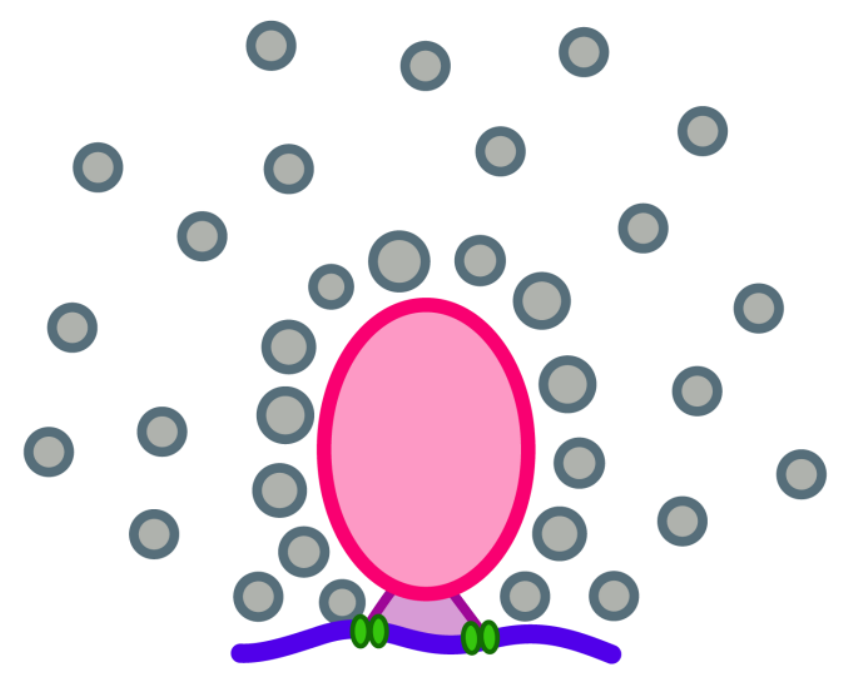

\title{
MORPHOLOGICAL STUDIES OF SYNAPTIC VESICLE RECYCLING AT THE INNER HAIR CELL RIBBON SYNAPSE
}

\section{DISSERTATION}

\author{
for the award of the degree \\ "Doctor rerum naturalium" \\ of the Georg-August-U niversität Göttingen
}

within the doctoral program "Sensory and M otor N euroscience"

of the Göttingen Graduate School for N eurosciences, Biophysics, and M olecular Biosciences

submitted by

Jana Kroll

from Neubrandenburg (Germany)

Göttingen 2018 


\section{Thesis Committee}

Dr. Ira M ilosevic ( $1^{\text {st }}$ reviewer, supervisor)

Synaptic V esicle Dynamics Group, European N euroscience Institute Göttingen

Prof. Dr. Tobias M oser ( $2^{\text {nd }}$ reviewer)

Institute for Auditory N euroscience and InnerEarLab, U niversity M edical Center Göttingen

Prof. Dr. Nils Brose

Department of M olecular Neurobiology, M ax-Planck-Institute for Experimental M edicine Göttingen

\section{FURTHER MEMBERS OF THE EXAMINATION BOARD}

Prof. Dr. Thomas Dresbach

Department of A natomy and Embryology, Center of A natomy, U niversity M edical Center Göttingen

PD Dr. Ellen Reisinger

Molecular Biology of Cochlear Neurotransmission Group, Otorhinolaryngology and InnerEarLab, University M edical Center Göttingen

Prof. Dr. Ralf H einrich

Deptartment of Cellular Neurobiology, Johann-Friedrich-Blumenbach-Institutefor Zoology and A nthropology, Georg-A ugust U niversity Göttingen 


\section{TABLE OF CONTENTS}

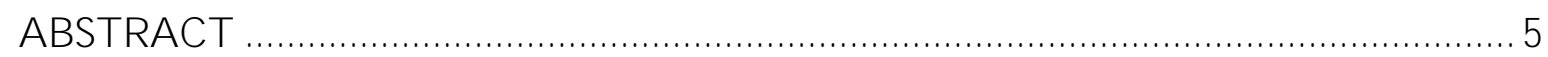

INTRODUCTION

1.1 Inner Hair Cells - an Intriguing Model System

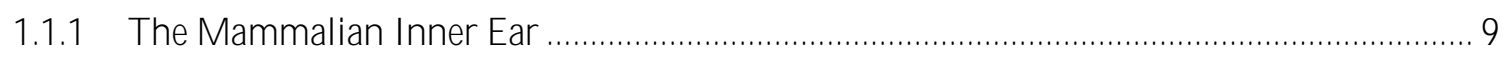

1.1.2 Inner Hair Cells Show a Polarized Cellular Organization .................................................... 11

1.2 What Makes Ribbon Synapses so Special? ............................................................... 14

1.2.1 The General Architecture of Synapses ......................................................................... 14

1.2.2 Peculiarities of Inner H air Cell Ribbon Synapses.............................................................. 14

1.2.2.1 Adjusted Vesicle Pool Organization in Inner Hair Cells ............................................. 15

1.2.2.2 The M olecular Composition of Inner H air Cell Ribbon Synapses............................. 17

1.3 IH C Synapses Show A daptive Neurotransmission ...................................................... 21

1.3.1 A Comparison of Exocytosis at CNS and IHC Synapses.............................................. 21

1.3.1.1 SN ARE-mediated Exocytosis Is Prevalent in N eurons but not in IHCs....................21

1.3.1.2 Release M echanisms at Central Synapses and Ribbon Synapses ............................... 22

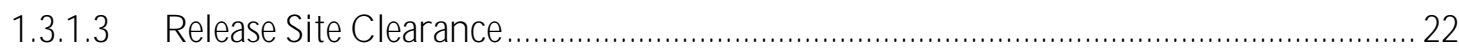

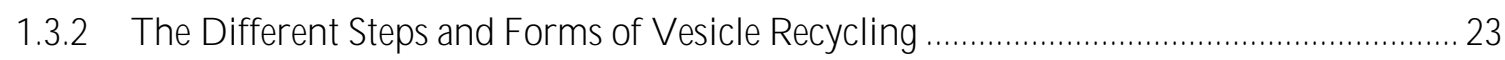

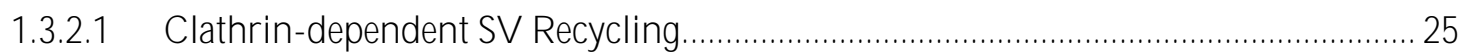

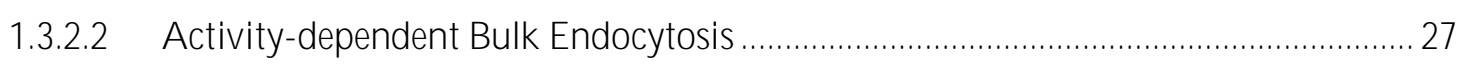

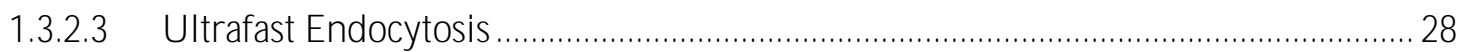

1.3.3 Endophilin-A and AP180 - Key Players in Synaptic Transmission ....................................2 29

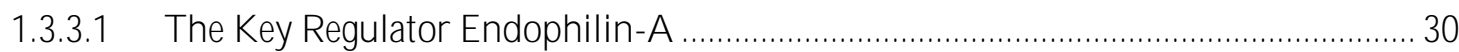

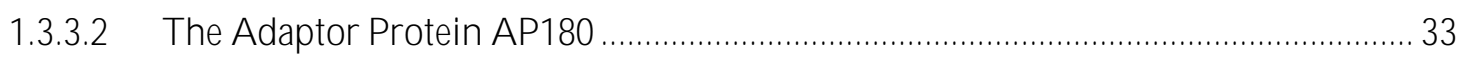

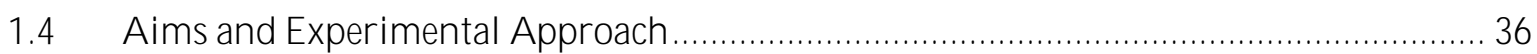

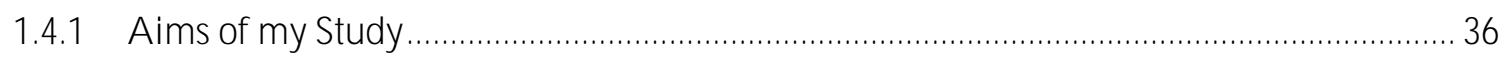

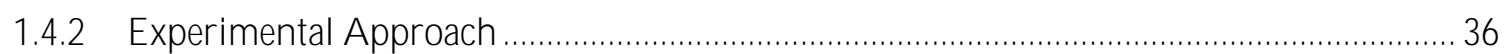


CHAPTER ONE

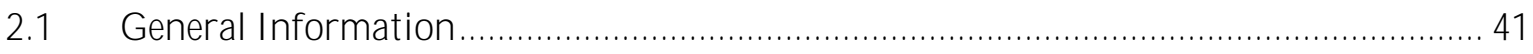

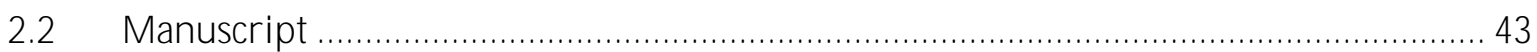

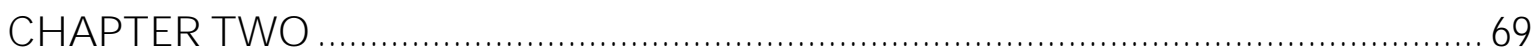

3.1 General Information ........................................................................................... 71

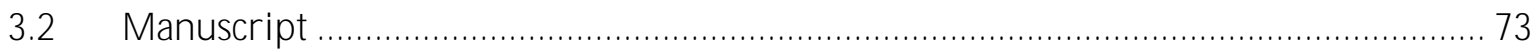

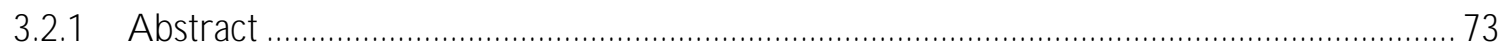

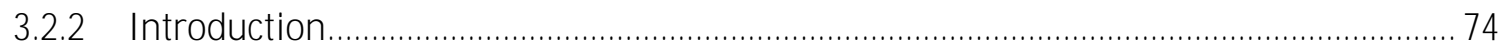

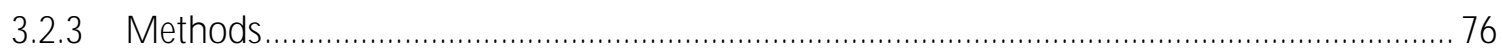

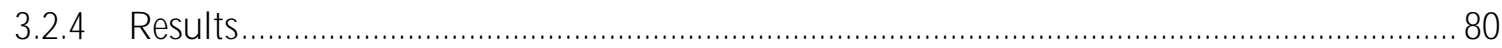

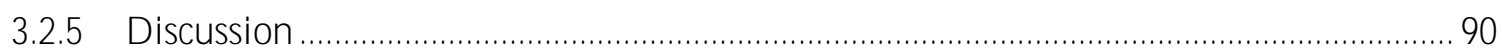

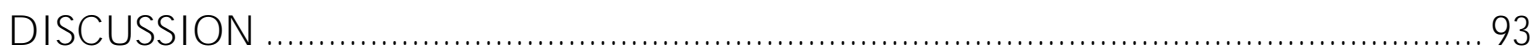

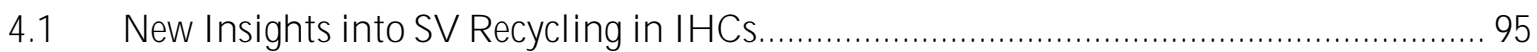

4.1.1 Endophilin and AP180 are Involved in Clathrin-mediated Endocytosis in IH Cs.............96

4.1.1.1 AP180 has a Role in Clathrin Recruitment in IHCs..................................................97

4.1.1.2 Endophilin is Required for Fission and Uncoating in IHCS ..................................... 98

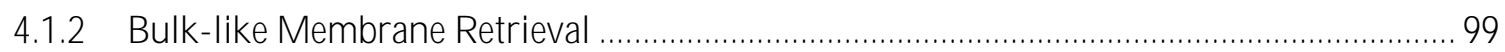

4.1.2.1 Bulk Retrieval M ay Sufficiently Recover M embranes in A bsence of AP180............ 100

4.1.2.2 Endophilin M ay Have a Role in Clathrin-independent Endocytosis in IH Cs......... 100

4.1.3 AP180 and Endophilin are Involved in SV Reformation ............................................. 102

4.1.4 Do IHCs N eed Clathrin-mediated Endocytosis? ............................................................ 103

4.2 AP180 and Endophilin Act Beyond SV Recycling

4.2.1 AP180 M ay Play a Role D ownstream of Docking in IH CS .............................................. 107

4.2.2 Endophilin Regulates the Size of Cav 1.3 Clusters and Interacts with Otoferlin............... 109

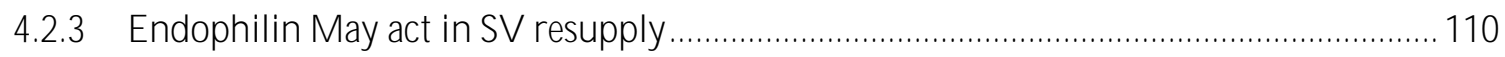

4.3 A Putative M odel for Synaptic T ransmission in IH C S........................................ 112

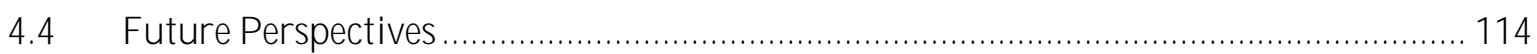




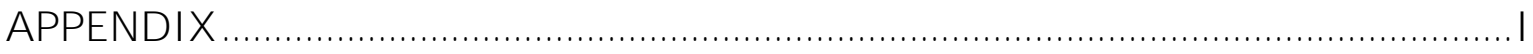

a) Additional M aterial for the Endophilin-A Study.......................................................... i

b) Additional M aterial for the AP180 Study .....................................................................

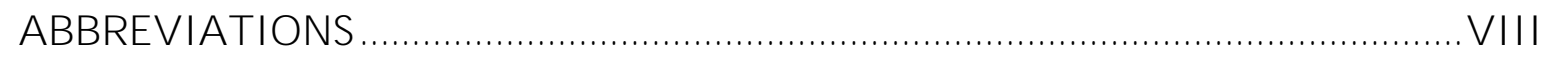

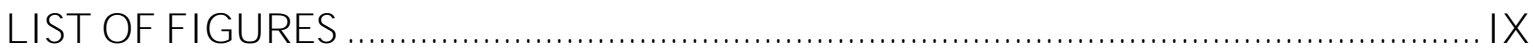

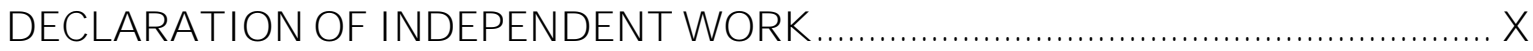

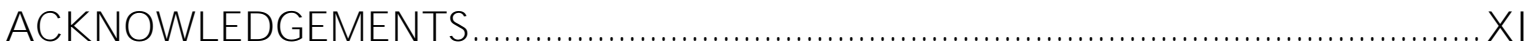




\section{ABSTRACT}

Within the mammalian inner ear, sensory inner hair cells (IHCS) of the organ of Corti are required to transduce sound waves into electrical signals. Specialized synapses at the IHC basal pole, so-called ribbon synapses, facilitate the vivid release of the neurotransmitter glutamate over long time periods. Not only the exocytic release machinery but also the organization of the synaptic vesicle (SV) pools are adapted in these cells. SV release in IHCs is further balanced by robust membrane retrieval and SV reformation. Even though fast and slow forms of endocytosis have been described, to date, little is known about the molecular entities regulating SV recycling in IHCS.

In the here presented two studies, I examined the roles of two key players in neuronal endocytosis, namely endophilin-A1-3 and AP180, in IHC synaptic transmission. Combining various methodologies, I could show together with my collaborators, that both proteins seem to have several functions along the SV cycle. Both, endophilin-A and AP180, are involved in clathrin-dependent processes in IHCs: while AP180 recruits clathrin, endophilin-A regulates the fission and uncoating of clathrin-coated pits and vesicles. Especially for the clathrin-dependent reformation of SV s from endosome-like vacuoles (ELVs), the actions of those two proteins appear rate-limiting. The two proteins endophilin-A and AP180 seem further required for the tight coupling of exo- and endocytosis. We could show that absence of endophilin-A results in impaired sustained exocytosis, which points towards a deficit in SV replenishment or SV recruitment to the ribbon, or to impaired release site clearance. In either of these processes, endophilin-A may interact with otoferlin. Absence of AP180 resulted in a defect downstream of docking, which we attribute to a function of AP180 in release site clearance. Finally, we could for the first time show that endophilin-A functionally modulates presynaptic $\mathrm{Ca}^{2+}$-channels and promotes $\mathrm{Ca}^{2+}$-channel clustering at IHC active zones.

Taken together, these two studies on the endocytic proteins endophilin-A and AP180 demonstrate that the different processes mediating the SV cycle in IHCS are tightly coupled and balanced. H owever, they also illustrate the robustness of the SV recycling and transmitter release of the first auditory synapse towards molecular disruption of genes coding for endocytic proteins, as none of the examined mouse mutants exhibited severe hearing deficits. 


\subsection{InNer HAIR CELLS - AN INTRIGUING MOdel SYSTEM}

A key characteristic of every living individuum is the ability to interact with its environment. Particularly in animals, this interaction does not only include the sensation of stimuli, but also communication with each other. For this purpose, environmental information is detected through sensory cells, e.g. retinal or inner ear cells, transduced into chemical or electrical signals, and transferred into the respective brain areas. Synapses formed between sensory cells and neurons, or between different neurons, form the basis for signal transmission within the sensory system. At these synapses, neurotransmitter-filled synaptic vesicles (SV s) undergo a cycle of release and recycling: Exocytosis at the presynaptic active zone (AZ) is induced by cell depolarization and results in the integration of the SV membrane into the cellular plasma membrane. This process goes along with the release of neurotransmitters into the synaptic cleft formed between pre and postsynapse. Exocytosis is accompanied by SV recycling, which includes the internalization of membrane (endocytosis) and the reformation of new SV s. Endocytosis is not only required to balance the increase in the cell surface following exocytosis, but also to clear release sites at the AZ from vesicular proteolipids. M oreover, the endocytosed membrane material builds the basis for newly formed SV s. These general steps within the SV cycle are conserved between different cell types and within different species. Themolecular composition and architecture of synapses, though, are heterogenous within the animal body and individually adapted to the specific demands of each respective synapse. Oneexamplefor such a specialization aretheso-called ribbon synapses within inner hair cells (IHCs) of the inner ear. In the first part of my introduction, I will summarize anatomical features of IHCS, followed by an extensive morphological and functional characterization of their ribbon synapses. In the third part of my introduction, I will compare synaptic transmission in neurons and in IHCS. Especially in comparison to neurons, IHCs serve as an interesting model system for exo- and endocytic processes: here, synaptic transmission is adapted to the edge of synaptic capabilities. Beyond that, understanding molecular processes in IHCs provides a basis for the development of therapeutic strategies for the treatment of hearing impairments associated with the inner ear.

\subsubsection{THE MAMMALIAN INNER EAR}

The mammalian ear consists of the outer ear, the middle ear, and the inner ear (Fig. 1-1A). While the outer ear is required for the bundling and filtering of incoming sound pressure waves, the middle 
ear, harboring the ear drum and the ossicles, matches the different impedance of sound conduction in the air and the fluid-filled inner ear. Within the inner ear, the mechanical information is transduced into electrical signals, which are transmitted via auditory nerves formed by the bipolar spiral ganglion neurons (SGNs) and perceived by the respective brain areas.

A

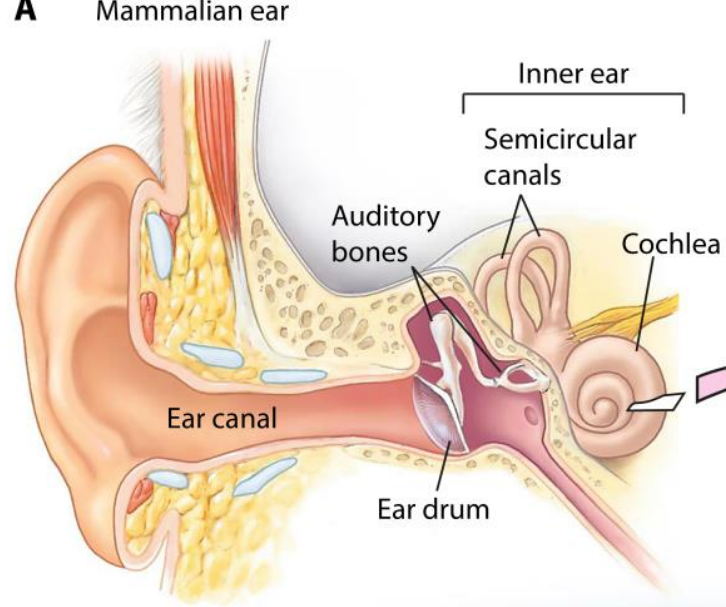

B Cochlea cross section

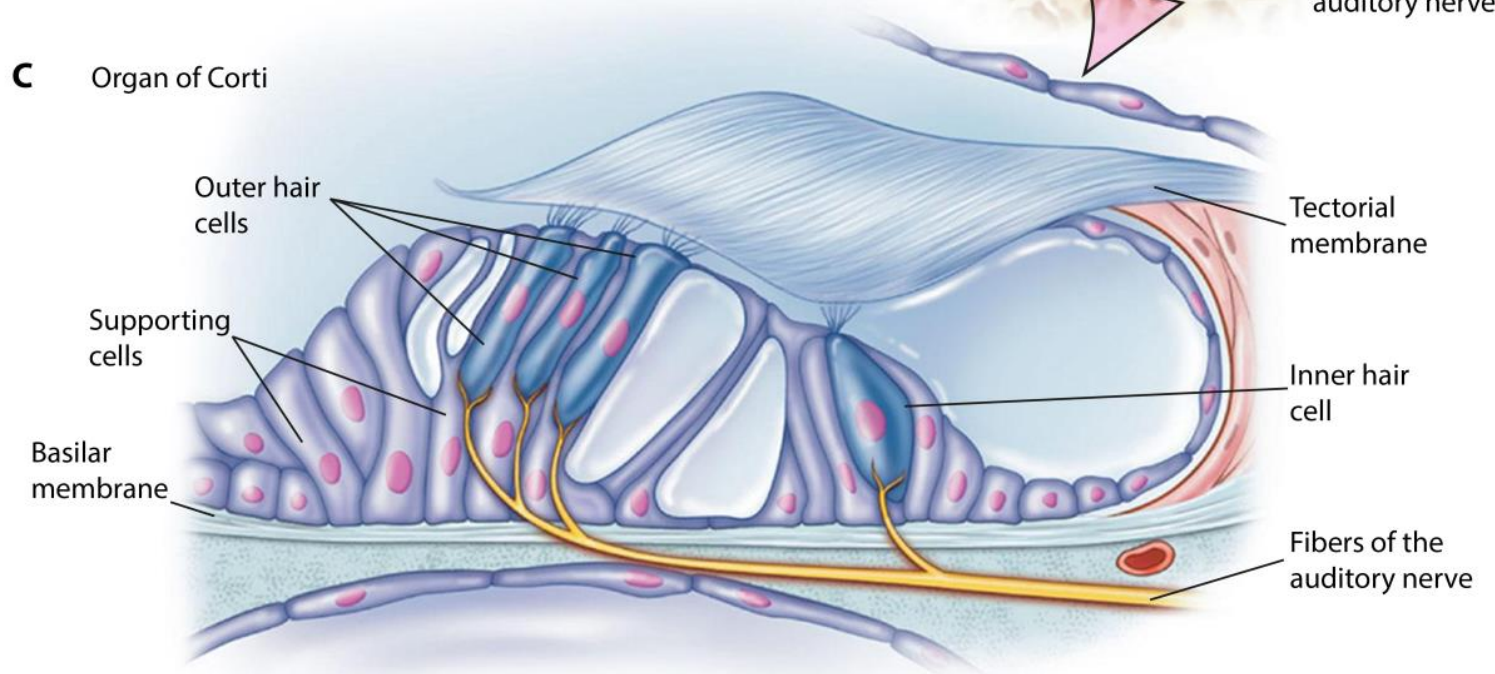

Fig. 1-1: The anatomy of the mammalian inner ear. (A) Overview of outer ear, middle ear and inner ear. (B) Cross-section through the cochlea with the three fluid-filled cavities, the organ of Corti on top of the basilar membrane, and the auditory nerve. (C) The organ of Corti contains three rows of OHCs with stereocilia embedded in the tectorial membrane, and one row of IHCS, as well as different types of supporting cells. Images modified from Pearson Education 2012.

The inner ear is formed by the vestibular apparatus with its three semicircular canals and two macula organs, which are required for balance, and by the cochlea, which is a bony structure resembling a snail's shell and displays theend-organ for hearing (Fig. 1-1A). The cochlea contains three fluid-filled compartments, which wind up along the cochlea: the scala media, scala vestibuli and scala tympani. (Fig. 1-1B). These cavities contain solutions of different composition. The scala vestibuli and scala 
tympani contain low- $\mathrm{K}^{+}$perilymph with a composition closely matching typical extracellular saline, whereas the scala media contains a high- $\mathrm{K}^{+}$fluid called endolymph. When sound enters the inner ear, the basilar membrane, which borders the scala media, vibrates in form of a travelling wave. This wave reaches its maximal amplitude at a specific region along the longitudinal axis of the cochlea depending on the frequency of the sound. This frequency-place- relationship is called tonotopy and leads to low-frequency signals having their maxima at the apex of the cochlea and high-frequency signals reaching their maxima at the base according to the varying micromechanical properties of the basilar membrane along the tonotopic axis. At this specific position, the organ of Corti, which is the sensory epithelium of the cochlea and located on top of the basilar membrane, processes the given sound stimulus (Fettiplace, 2017). The organ of Corti is formed by three rows of outer hair cells (OHCs) and one row of IHCs, as well as by different types of supporting cells (Fig. 1-1C). OH Cs are studded with stereocilia that project not only into the scala media but are, at least partially, also embedded into the tectorial membrane that covers theorgan of Corti. Sound-induced movements of basilar membraneand tectorial membrane relative to each other induce deflections of thestereociliar hair bundles of the OHCs. The OHCs now contract and expand in response to the sound stimuli, which further amplifies the relative movements of the basilar membraneand tectorial membraneand also the resulting radial flux of the endolymph between the tectorial membrane and the surface of the organ of Corti (Fettiplace, 2017). The flux now causes displacements of hair bundles of theIHCs and eventually results in the opening of mechano-electrical transduction channels located within the membrane of the stereocilia (Assad et al., 1991; Howard and Hudspeth, 1988). These nonselective cation channels show ultrafast activation and closing kinetics and provide graded cell depolarization or hyperpolarization through $\mathrm{K}^{+}$influx dependent on intensity and direction of stereociliar deflections (Beurg et al., 2006; Corey and Hudspeth, 1979; Fettiplace, 2017).

\subsubsection{InNer Hair Cells Show a POLARIZEd Cellular Organization}

Like in OHCS, hair bundles of IHCs are located at the top of the cells, whereas voltage-gated Cav 1.3 $\mathrm{Ca}^{2+}$-channels, which open upon depolarization of the cell, are exclusively found in the basal half of IHCs (see Fig. 1-2; Brandt et al., 2003; Platzer et al., 2000; Roberts et al., 1990). Generally, IHCs show a strong polarization along the apicobasal axis: Stereocilia at the apex of IHCs are anchored in the cuticular plate, which is an amorphous network of cytoskeletal proteins like actin (Slepecky and Chamberlain, 1985). Golgi complexes as well as LAMP1-positive structures likely displaying Iysosomes have exclusively been detected in the apical half of IHCs (Revelo et al., 2014; Siegel and 
Brownell, 1986). Even though endosome-like vacuoles (ELVs) can be observed in all parts of IHCs, markers for early endosomes (EEA 1) and late endosomes (syntaxin 16) have only been visible in the apical and nuclear region, but not beneath (Revelo et al., 2014).

Contrarily, synaptic transmission from IHCs to SGN s is restricted to the basal region of IHCs. N ot only neurotransmitter release, but also stimulus-evoked endocytic processes and SV reformation were detected in the IHC base (Kamin et al., 2014; Kantardzhieva et al., 2013; Revelo et al., 2014). $\mathrm{H}$ ere, the specialized ribbon synapses are located to facilitate the $\mathrm{Ca}^{2+}$-induced release of glutamate (see Fig. 1-2C). D epending on the tonotopic position along the basilar membrane, individual cochlear IH Cs harbor between 5 and 20 ribbon synapses (M eyer et al., 2009). Each of these synapses is thought to be innervated by a single afferent SGN (Liberman, 1978). After the onset of hearing at P12 in mice (M ikaelian and Ruben, 1965), usually one electron-dense structure named synaptic ribbon (see 1.2.2 Peculiarities of Inner Hair Cell Ribbon Synapses) can be detected per AZ, rarely two or even three (Sobkowicz et al., 1986; W ong et al., 2014). Interestingly, the size of synaptic ribbons and active zones as well as the $\mathrm{Ca}^{2+}$-influx and rates of spontaneous neurotransmitter release are heterogenous between individual AZs within the same IHC (Frank et al., 2009; M eyer et al., 2009; O hn et al., 2016). Likewise, SGN s that innervate the IHCs differ in their diameter (Liberman, 1982a; M erchan-Perez and Liberman, 1996; Ohn et al., 2016). Ribbon synapses containing a larger synaptic ribbon, more $\mathrm{Ca}^{2+}$-channels, and a high number of SV s are predominantly found at the modiolar side (facing the spiral ganglion), whereas smaller ribbons connected to a higher rate of spontaneous release are preferentially located at the pillar side (facing the OHCs) of IHCs (Frank et al., 2009; M eyer et al., 2009; O hn et al., 2016). It has been suggested that the heterogeneity of ribbon synapses and SG Ns is required for the encoding of different sound pressure levels (Liberman, 1982a; Liberman, 1982b; M erchan-Perez and Liberman, 1996; M oser and V ogl, 2016). 

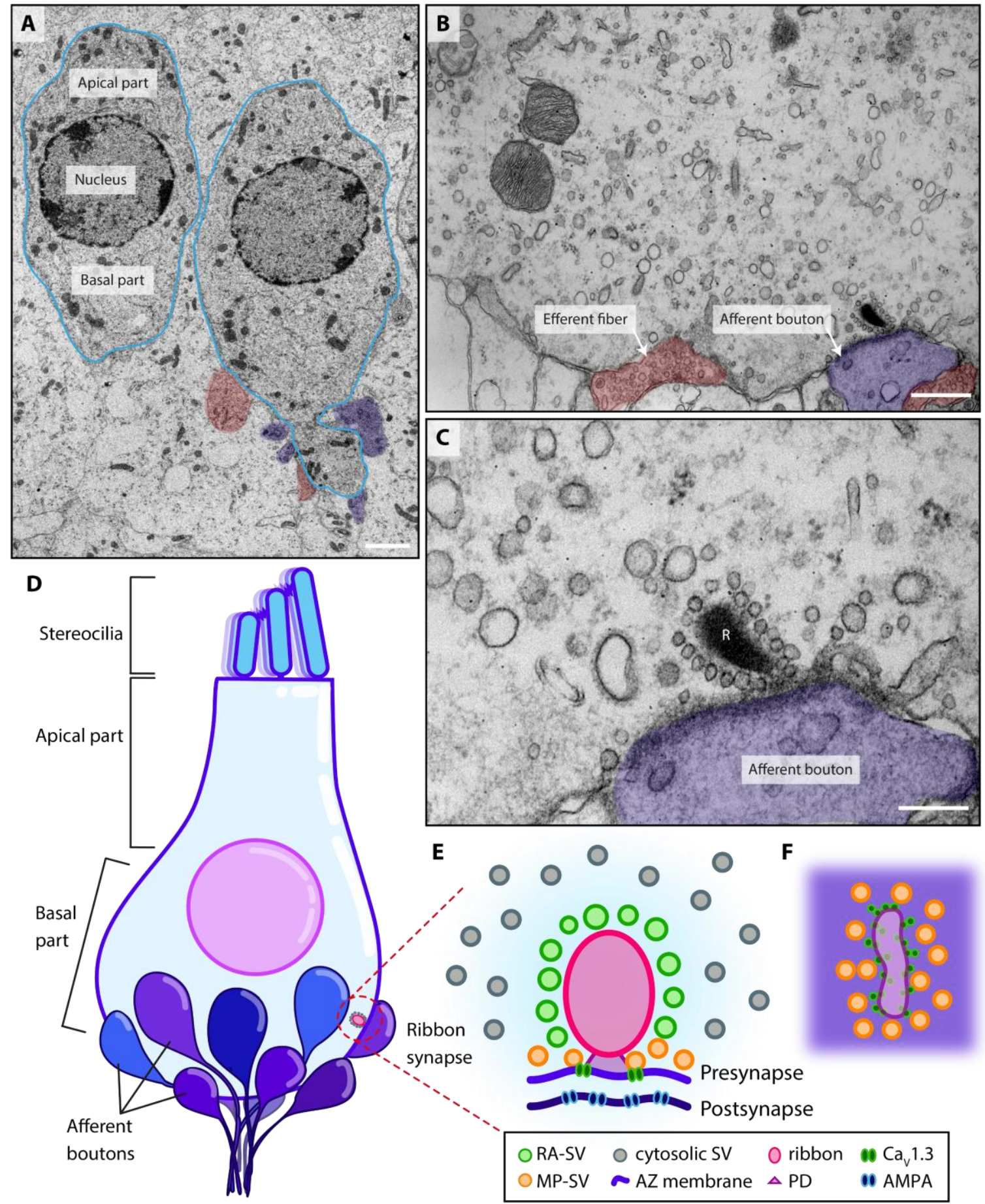

Fig. 1-2: Morphological characteristics of IHCs and ribbon synapses. (A) Overview of two IHCs. Cell borders are highlighted in blue, afferent (violet) and efferent (red) nerve terminals are shaded. Scale bar $2 \mu \mathrm{m}$. (B) Higher magnification of an IHC base containing a ribbon synapse. Scale bar $500 \mathrm{~nm}$. (C) Crosssection of a synaptic ribbon (R) surrounded by SVs opposed to an afferent bouton. Scale bar $200 \mathrm{~nm}$. (D-F) Schematic drawings of an IHC (D), a cross-section of a ribbon synapse (E) and the top view of an active zone without the ribbon (F). RA-SV ribbon-associated SV; MP-SV membrane-proximal SV; PD presysnaptic density; AMPA $\alpha$-amino-3-hydroxy-5-methyl-4-isoxazolepropionic acid receptor. 


\subsection{WHAT MAKES RIBBON SYNAPSES SO SPECIAL?}

\subsubsection{THE GENERAL ARCHITECTURE OF SYNAPSES}

Examining peculiarities of $\mathrm{IHC}$ ribbon synapses does not work without understanding the fundamental characteristics of synapses: A presynapse containing SV s to be released at the active zone, a postsynapse containing receptors for neurotransmitter perception, and a synaptic cleft. These features are conserved not only between different species from invertebrates to mammals, but also between different cell types. AZs of neuronal synapses, which are probably the best-understood type of synapses, are covered by small dense projections, which are in close proximity to $\mathrm{Ca}^{2+}$-channels and SV s (O heim et al., 2006; Zhai and Bellen, 2004). A meshwork of multi-domain proteins forms the cytomatrix at the active zone (CAZ) that is crucial for the maintenance of synaptic transmission (Südhof, 2012). Depending on the specific demands, architecture and molecular composition of synapses may differ enormously between different cell types and in different species, though. In invertebrates, a specialized, well-studied synapse can be found at neuromuscular junctions of Drosophila melanogaster. Instead of small dense projections, these synapses contain an electrondense three-dimensional structure extending from the AZ membrane called T-bar, which is formed by the protein bruchpilot and supports fast SV release (Kittel et al., 2006; W agh et al., 2006). In hair cells and retinal cells of vertebrates, e.g. of fishes, frogs, guinea pigs and mice, electron-dense presynaptic structures have likewise been detected that are named synaptic ribbons (D owling, 1968; Flock and Duvall, 1965; Gray and Pease, 1971; Sjostrand, 1958; Smith and Sjöstrand, 1961).

\subsubsection{PeCuliarities of InNer Hair Cell RibBon SynapSeS}

Synaptic ribbons are the hallmark of ribbon synapses and are mainly composed of the protein RIBEYE (see 1.2.2.2 The M olecular Composition of Inner Hair Cell Ribbon Synapses). A halo of SVs is tethered to the ribbon as well as to the AZ membrane in close proximity to the ribbon (see Fig. 1$2 \mathrm{E}$ and F; Frank et al., 2010; M atthews and Fuchs, 2010; Zhai and Bellen, 2004). In saccular hair cells, ribbons arespherical while they are reminiscent of a horse shoe in murine retinal photoreceptor cells (Dick et al., 2003; Lenzi and von Gersdorff, 2001; Zhai and Bellen, 2004). In cochlear IHCs, ribbons are oval- or droplet-shaped in cross-section and elongated in the longitudinal direction (Sobkowicz et al., 1982; W ong et al., 2014). 
A number of putative functions for ribbon synapses have been postulated not only in cochlear $\mathrm{IHCS}$, but also in retinal photoreceptor or bipolar cells, as well as in zebrafish hair cells: (i) Tethering of a large SV pool in close vicinity to the AZ, the ribbon could play an essential role in the replenishment of SV s in the course of continuous exocytosis (Becker et al., 2018; Frank et al., 2010; Jean et al., 2018; M axeiner et al., 2016; Snellman et al., 2011). Whether SV s are passively delivered to the release sites via diffusion, also termed "crowd surfing" (Graydon et al., 2014), or if the ribbon actively regulates SV resupply, is not fully understood yet (Lenzi and von Gersdorff, 2001; M axeiner et al., 2016). (ii) At photoreceptor ribbon synapses, a direct involvement of the ribbon in priming of SV s, or at least in the recruitment of priming factors, has been suggested (Snellman et al., 2011). Even though functions of the ribbon in SV priming in IHCS still lack verification, it could be shown that (iii) ribbons likely facilitate exocytosis through the provision of multiple release sites and co-localizing of SV s to $\mathrm{Ca}^{2+}$-channels (Edmonds, 2004; Fuchs, 2005; Glowatzki and Fuchs, 2002; Khimich et al., 2005; Li et al., 2009). (iv) A bsence of the ribbon resulted in impaired clustering of $\mathrm{Ca}^{2+}$-channels (Frank et al., 2010; Jean et al., 2018; Khimich et al., 2005; Sheets et al., 2011). Hence, the ribbon seems to also be involved in the organization and/or functional modulation of $\mathrm{Ca}^{2+}$-channels at the AZ. (v) Last but not least, the ribbon may form a $\mathrm{Ca}^{2+}$ diffusion barrier, thus generating very high local $\mathrm{Ca}^{2+}$ concentrations (Graydon et al., 2011).

\subsubsection{Adjusted Vesicle Pool Organization in InNer Hair Cells}

Neurotransmitter release in IHCs is supposed to be highly organized, meaning that different stimuli can induce exocytosis of distinct SV pools. In neuronal synapses, three main SV pools have been characterized morphologically and physiologically (Elmqvist and Quastel, 1965; Liley and North, 1953; N eher, 1998; Rizzoli and Betz, 2005): (i) The readily-releasable pool (RRP) is located just above the AZ plasma membrane and can be released as soon as a stimulus reaches the nerve terminal (Rosenmund and Stevens, 1996; Schikorski and Stevens, 2001; Schneggenburger et al., 1999). (ii) Continuous stimulation leads to the depletion of the RRP, which is subsequently refilled by SV s from the recycling pool (also termed slowly-releasable pool; SRP) located in the vicinity of the AZ membrane (Harata et al., 2001; Neher, 1998; Rizzoli and Betz, 2005). (iii) Strong stimulation ultimately induces the mobilization of the cytosolic reserve pool of SV s for release (H arata et al., 2001; Neher, 1998; Rizzoli and Betz, 2005). W hile the reserve pool is sparsely utilized in neuronal synaptic 
transmission under physiological conditions, other synapses may more frequently make use of this SV pool (Denker and Rizzoli, 2010; Truckenbrodt et al., 2018).

Electrophysiological recordings in IHCs revealed two phases of SV exocytosis: a short phase with exponential release and a later phase with a continuous, linear rate of release (M oser and Beutner, 2000; Rutherford and Roberts, 2006). The initial phase that can already be induced by brief depolarizations (upto $20 \mathrm{~ms}$ ) likely reflects the RRP of SV s and comprises one to two dozens of SV s per AZ (M oser and Beutner, 2000; Pangršič et al., 2010). Several studies, not only in cochlear IHCS but also in saccular hair cells, have suggested that the RRP is formed by SV s lining up directly below the ribbon base (Frank et al., 2010; Lenzi et al., 1999; Moser and Beutner, 2000; Rutherford and Roberts, 2006; Sobkowicz et al., 1982). The SV s forming the physiological RRP have been suggested to be part of the morphological membrane-proximal SV pool (MP-SV) at IHC ribbon synapses (Chakrabarti et al., 2018; Frank et al., 2010; Jung et al., 2015a). Due to the length of tethers, i.e. filamentous connectors (also see below), between SV s and the AZ membrane examined via highpressure freezing (H PF), a max. distance of M P-SV s to the AZ membrane of roughly $50 \mathrm{~nm}$ has been established (Chakrabarti et al., 2018; Jung et al., 2015a). U pon stimulation, the M P-SV pool was shown to be more strongly depleted at different ribbon synapses than the SV s around the ribbon (Frank et al., 2010; Lenzi et al., 2002; Pangršič et al., 2010). Stronger depolarizations (Ionger than $50 \mathrm{~ms}$ ) were shown to induce the sustained phase of release, which is required for processing continued sound stimuli (Pangršič et al., 2010). SV s required for the tonic release of neurotransmitters have been suggested to be recruited to the release sites from potential SV pools around the ribbon, in direct vicinity to the ribbon, or even within the cytosol in ribbon proximity at different ribbon synapses (Frank et al., 2010; Glowatzki and Fuchs, 2002; Lenzi et al., 1999; M oser and Beutner, 2000; Schnee et al., 2005). Previous studies further indicated that the SV s around the ribbon are released prior to cytosolic SV s (Frank et al., 2010; Jean et al., 2018; Lenzi et al., 1999; Lenzi et al., 2002; Schnee et al., 2005). Although an allocation of SV s within the IHC base to SV pools reflecting the recycling pool and the reserve pool in neurons has not been possible, a morphological pool of ribbon-associated SV s (RA-SV s) in cochlear IH Cs has been defined (Chakrabarti et al., 2018; Frank et al., 2010; Jung et al., 2015a; W ong et al., 2014). This RA-SV pool harbors SV s in the first row around the ribbon, usually with a tethering filament formed between SV and ribbon.

Not only at ribbon synapses, but also at central synapses, SV s are linked to each other as well as to scaffolding proteins and the AZ membrane via filamentous tethers. Filaments connecting SV s with 
each other (interconnectors) support the mobilization of SV s as well as their stay within defined SV pools (Cole et al., 2016; Fernández-Busnadiego et al., 2010; Hallermann and Silver, 2013; Siksou et al., 2007). Likewise, tethers formed between SV s and the ribbon in IHCs could be required for the organized, continuous release of neurotransmitters (Chakrabarti et al., 2018). To date, the protein composition of filaments between SVs and the ribbon in IHCs, as well as of interconnectors, is elusive. Tether formation between SV s and the AZ membrane is supposed to be an essential step in the initiation of SV exocytosis (Chakrabarti et al., 2018; I mig et al., 2014). Thesetethers vary in length and morphology, whereby more multi-tethered SV s have been observed in IHCs after stimulation (Chakrabarti et al., 2018). It has therefore been proposed that the formation of multiple short tethers between SV s and AZ precedes docking and fusion, as previously described in neurons (Chakrabarti et al., 2018; Cole et al., 2016; Fernández-Busnadiego et al., 2013). In neurons and at IHC ribbon synapses, RIM s have been suggested to play a role in the tethering of SV s to the AZ (Betz et al., 2001; Fernández-Busnadiego et al., 2013; I mig et al., 2014; J ung et al., 2015a). Whether also SN ARE (soluble $\mathrm{N}$-ethylmaleimidesensitive factor (NSF) attachment protein receptors) protein interaction is part of the tethering process at least at central synapses, is still under debate (Fernández-Busnadiego et al., 2010; I mig et al., 2014). A Ithough IH C s seem to operate without neuronal SN ARE proteins (see 1.3.1 A Comparison of Exocytosis at CNS and IHC Synapses; Nouvian et al., 2011), a partial requirement of the IHC exocytic protein otoferlin for the formation of short tethers has been described (Vogl et al., 2015).

\subsubsection{The Molecular Composition of InNer Hair Cell RibBon Synapses}

Ribbon synapses, as well as synapses at neuronal nerve terminals, are designed for $\mathrm{Ca}^{2+}$-induced neurotransmitter release. Developmentally, IHCs and neurons are formed from different embryonic compartments, though. IHCs derivefrom placodes formed within the epithelial part of the ectoderm, whereas neurons of the central nervous system originate from theneural tube (Graham and Shimeld, 2013). Therefore, it is plausible that the molecular composition of the $A Z$ and of the release machinery partially differ between IHC ribbon synapses and neuronal synapses.

\section{SCAFFOLDING PROTEINS}

The cytomatrix protein RIBEYE represents the main organizational compound of synaptic ribbons not only at IHC ribbon synapses but also in the retina. RIBEYE consists of an A domain involved in 
the assembly of the ribbon and a B domain with enzymatic activity (Khimich et al., 2005; Schmitz et al., 2000; Schwarz et al., 2011). The B domain is structurally almost identical to the transcription repressor C-terminal binding protein 2 (CtBP2) and may facilitate the tethering of SV s to the ribbon (Schmitz et al., 2000). Knockout of the RIBEYE A domain was found to severely impair synaptic function in the retina, whereas IHC synaptic transmission was shown to be rather mildly affected (Becker et al., 2018; Jean et al., 2018; M axeiner et al., 2016). I mpressive developmental modifications - including the re-shaping of the AZ with several small presynaptic densities that were shown to tether SV s in RIBEYE-knockout (KO) IHCs - may here compensate in part for the absence of the synaptic ribbons (Jean et al., 2018).

The scaffolding protein bassoon has originally been described as one major component of the CAZ at central synapses (Südhof, 2012; tom D ieck et al., 1998). I mmunogold electron microscopy revealed that bassoon is also present at IHC and retinal AZs and here forms a component of the presynaptic density anchoring the synaptic ribbon (Dick et al., 2003; tom Dieck et al., 2005; W ong et al., 2014). Consequently, ribbons are not attached to the $\mathrm{Z} Z$ in bassoon-KO mice but free-floating in the cytosol (Dick et al., 2003; Frank et al., 2010; Khimich et al., 2005). In addition, bassoon was shown to be required for the stabilization of the RRP and for the clustering of $\mathrm{Ca}^{2+}$-channels (Frank et al., 2010; Jean et al., 2018; Jing et al., 2013; Khimich et al., 2005; N eef et al., 2018). A t central synapses, bassoon and piccolo mostly act together in the assembly of the AZ and in scaffolding, as well as in SV replenishment (Butola et al., 2017; Fenster et al., 2000; M endoza Schulz et al., 2014; Südhof, 2012; tom Dieck et al., 1998), but seem to have distinct functions at ribbon synapses. At retinal and IHC ribbon synapses, only a truncated form of piccolo called piccolino could be substantiated, which is missing the C-terminal binding sites for bassoon and RIM (Regus-Leidig et al., 2013). Immunogold labeling revealed the localization of piccolino exclusively around the ribbon (reminiscent of RIBEYE staining); and knockdown of piccolo/ piccolino in the retina was shown to result in impaired ribbon formation (Limbach et al., 2011; Regus-Leidig et al., 2014). It remains to be investigated if piccolino is involved in ribbon assembly, if it has a function in organizing CAZ or fusion proteins, or if piccolino contributes to the formation of tethers. Based on the localization, piccolino could be involved in tethering SV s to the ribbon. In contrast, RIM s were shown to play a role in the tethering of SV s to the AZ (Jung et al., 2015a). M oreover, RIM s can interact with Cav $1.3 \mathrm{Ca}^{2+}$-channels and, accompanied by RIM - binding protein (RIM -BP), regulate the clustering of these channels (Jung et al., 2015a; Krinner et al., 2017, 201; Picher et al., 2017). 


\section{CALCIUM ChannelS}

In IHCs, Cav1.3 is the almost exclusively present subtype of $\mathrm{Ca}^{2+}$-channels (Brandt et al., 2003; Platzer et al., 2000). A pprox. $50-80 \%$ of these channels in a cell form dense clusters at the $A Z$, with each cluster consisting of 80-120 individual channels on average (Brandt et al., 2005; N eef et al., 2018; Roberts et al., 1990; W ong et al., 2014; Zampini et al., 2013). Still, numbers of channels and cluster lengths can vary greatly depending on the localization of the AZ within the IHC (Frank et al., 2010; Neef et al., 2018; Ohn et al., 2016; Wong et al., 2014). In general, $\mathrm{Ca}^{2+}$-channels are organized in a comparably higher density within the clusters at IHC ribbon synapses than at central synapses, and provide ultrafast activation and very slow inactivation kinetics (Neef et al., 2018; Zampini et al., 2013). M ostly, these clusters form a stripe- or double stripe-like pattern that closely conforms the distribution of bassoon (Frank et al., 2010; N eef et al., 2018; W ong et al., 2014). Ca ${ }^{2+}$-channels are tightly coupled to SV s, and it seems as if a single channel controls the release of one SV (Brandt et al., 2005; W ong et al., 2014).

\section{The Multi-C 2 DOMain Protein OtOferlin}

In IHCS after the onset of hearing, $\mathrm{Ca}^{2+}$-sensing for exocytosis apparently does not require the neuronal $\mathrm{Ca}^{2+}$ sensor synaptotagmin-1 (Beurg et al., 2010; Reisinger et al., 2011; Safieddine and Wenthold, 1999). However, the IHC-specific protein otoferlin harbors 6-7 $C_{2}$ domains, which are structurally related to those of synaptotagmin-1 (Helfmann et al., 2011; Pangršič et al., 2012; Reisinger et al., 2011; Roux et al., 2006). A part from the $C_{2}$ domains, most of which can bind $\mathrm{Ca}^{2+}$, otoferlin possesses a C-terminal trans-membrane domain, as well as a Fer domain and a coiled-coil domain (Johnson and Chapman, 2010; Pangršič et al., 2012; Roux et al., 2006). Even though otoferlin has been suggested to act as a Ca+2+-sensor for exocytosis in IHCs (Johnson and Chapman, 2010; M ichalski et al., 2017; Roux et al., 2006), transgenic expression of synaptotagmin-1 in otoferlin-KO mice could not restore exocytosis (Reisinger et al., 2011). These and further studies on otoferlin-KO mice led to the assumption that otoferlin is moreover crucial for subsequent steps of exocytosis like SV tethering and priming (Pangršič et al., 2010; Roux et al., 2006; Strenzke et al., 2016; Vogl et al., 2015). Even though otoferlin seems not to be a main component of short tethers, it may aid their formation - potentially as a priming factor (Vogl et al., 2015). In this regard, multiple binding sites for $\mathrm{Ca}^{2+}$-channels, phospholipids and adaptor proteins may allow otoferlin to link fusion-competent SV s and exocytic proteins in close proximity to the release sites (H ams et al., 2017; Padmanarayana 
et al., 2014; Ramakrishnan et al., 2009). A lternatively or in addition, functions of otoferlin in SV replenishment and/ or release site clearance have been suggested (Chakrabarti et al., 2018; J ung et al., 2015b; Pangršič et al., 2010). Direct interactions of otoferlin and the adaptor protein AP-2 have been interpreted as indicators for a role of otoferlin in SV reformation, in endocytosis and/ or in the coupling of exocytosis and endocytosis (D uncker et al., 2013; Jung et al., 2015b; Strenzke et al., 2016). 


\subsection{IHC SyNAPSES ShOW AdAPTIVE NEUROTRANSMISSION}

To sustain transmission rates of up to hundreds of $\mathrm{Hz}$ as long as a sound stimulus continues, in combination with high temporal precision, ultrafast exocytosis needs to be perpetuated by extremely efficient SV replenishment and tightly balanced by compensatory membrane retrieval and SV reformation (Beutner et al., 2001; Jung et al., 2015b; Lenzi et al., 2002; M oser and Beutner, 2000; N eef et al., 2014; Parsons et al., 1994; Siegel and Brownell, 1986; Trapani et al., 2009). W hile mechanisms and molecular entities of endocytosis seem to be at least partially preserved between IHCs and neurons, the exocytic release machinery in IHCs is unique in many aspects.

\subsubsection{A COMPARISON OF EXOCYTOSIS AT CNS AND IHC SYNAPSES}

\subsubsection{SNARE-Mediated EXocytosis Is PreVALENT In NeURONS BUt nOT IN IHCS}

A key feature of central synapses is the SNARE-mediated release of neurotransmitters. SNARE proteins are subdivided into two sub-groups: v-SNA REs like vesicle-associated protein 2 (VAM P2) are located at the vesicular membrane whereas t-SNARES like synaptosomal-associated protein 25 (SNAP-25) and syntaxin-1 are connected to the target membrane/ the AZ (reviewed in Südhof and Rothman, 2009). VAM P2, syntaxin-1 and SNAP-25 together form the trans-SN A RE complex, which brings the membranes of the AZ and of the SV tightly together (Jahn and Fasshauer, 2012; Söllner et al., 1993; Sutton et al., 1998). Once those membranes are in direct contact, they are defined as morphologically docked (Verhage and Sørensen, 2008). Whether SV priming is a distinct step following docking or whether docking and priming are morphologically and physiologically indistinguishable processes is currently under debate(Imig et al., 2014; V erhage and Sørensen, 2008).

Electron tomography has revealed that SV s are brought into close proximity to the AZ via tethers in neurons as well as in IHCs (Chakrabarti et al., 2018; Fernández-Busnadiego et al., 2013). H owever, the docking process in IHCS is apparently orchestrated by a different set of proteins, as synaptic transmission in IHCs was shown to be insensitive towards the treatment with neurotoxins which cleave the neuronal SN A RE proteins SN AP-25, syntaxin-1 and VAM P1-3 (N ouvian et al., 2011). In the same study, immunohistochemical staining revealed the presence of all these SN ARE proteins in efferent synapses near the IHCs but not at the IHC synapse. Even though mRNA of SNAP-25, syntaxins and VAMP1-3 could be detected in IHCS, the authors questioned their physiological 
relevance (Nouvian et al., 2011; Safieddine and Wenthold, 1999). Consequently, IHC exocytosis either works completely independent of SNARE complex formation, or it is mediated by nonneuronal SNARE proteins. M oreover, different studies were showing that complexins, M unc13, and CAPS, which regulate docking, priming, and fusion in neurons (Brose, 2008; Chen et al., 2002; $\mathrm{H}$ ata et al., 1993), do not operate at IH C ribbon synapses (Strenzke et al., 2009; V ogl et al., 2015).

\subsubsection{Release MeChanisms at Central Synapses and RibBon SynapSeS}

In neurons, the $\mathrm{Ca}^{2+}$-dependent trans-SNARE complex formation goes along with the release of energy that is thought to finally initiate the fusion of SV membrane and AZ membrane via formation of a fusion pore (W iederhold and Fasshauer, 2009). The subsequent full integration of SV membrane into the AZ has been the favored model in neurons as well as at IHC ribbon synapses. Particularly in IHCS, the simultaneous fusion of multiple SV s facilitated through the ribbon could serve as an explanation for fast and strong neurotransmission (Glowatzki and Fuchs, 2002). An alternative mechanism is the univesicular release with a strong postsynaptic AM PA receptor complement, whereby the repetitive opening and closing of the fusion pore, called fusion pore flickering, might explain the occurrence of multiphasic excitatory postsynaptic currents (EPSCs; Chapochnikov et al., 2014). During fusion pore flickering, only parts of the neurotransmitter are released during every opening, before the SV is finally fully integrated into the AZ membrane (Alés et al., 1999). Fusion pore flickering was initially examined in chromaffin cells (Chow et al., 1992; Zhou et al., 1996), but has also been proposed to occur in IHCs (Chapochnikov et al., 2014). Here, the univesicular release has been proposed as an alternative explanation for differences between individual release events at an AZ (Chapochnikov et al., 2014). Whether the repetitive opening and closing of a fusion pore plays a physiological rolein neurotransmission at central synapses is still under debate(Fernández-Alfonso and Ryan, 2004; Klingauf et al., 1998; Rizzoli and Jahn, 2007).

\subsubsection{ReleAse Site Clearance}

A fter the full integration of SV membraneinto the AZ plasma membrane, the release machinery, and in particular the trans-SN ARE complex in neurons, has to be disassembled (Clary et al., 1990; Söllner et al., 1993). While SN AP-25 and syntaxin- 1 seem to stay in or at the plasma membrane, VAM P2 is supposed to be retrieved and re-used in future rounds of SV exocytosis. Not only SNAREs but also other proteins involved in exocytosis have to be retrieved from the AZ membrane. In this process 
called release site clearance, exocytic key proteins and adaptor proteins, which have originally been characterized as endocytic proteins, act together in the tight coupling of exo- and endocytosis (H aucke et al., 2011; N eher, 2010). For instance the neuronal assembly protein 180 (AP180) and its ubiquitously expressed homolog CALM (clathrin assembly lymphoid myeloid leukemia), were shown to specifically target VAMP2 and other members of the VAM P family, as more extensively recapitulated in chapter 1.3.3.2 The Adaptor Protein AP180 (Koo et al., 2011; Koo et al., 2015). A nother adaptor protein, AP-2, was shown to play a role in release site clearance in neurons via interaction with synaptotagmin-1 (Haucke et al., 2000; Kononenko et al., 2013) and in IHCs via interaction with otoferlin (Duncker et al., 2013; Jung et al., 2015b). In AP-2 $\mu$ KO mice, otoferlin levels were shown to be more than $70 \%$ reduced and the remaining otoferlin staining was found relatively more prominent at the plasma membrane (Jung et al., 2015b).

\subsubsection{THE DIFFERENT STEPS AND FORMS OF VESICLE RECYCLING}

N ot only proteins and proteolipids at the AZ surface finally induce endocytosis. Another factor that serves as an initiator for membrane internalization is the increase in membranetension following SV fusion events (Kononenko and Haucke, 2015). Furthermore, $\mathrm{Ca}^{2+}$-binding proteins like calmodulin as well as phospholipids binding to endocytic proteins have been discussed to induce endocytosis in neurons and potentially also at non-neuronal synapses (Dittman and Ryan, 2009; Maritzen and H aucke, 2018; Puchkov and H aucke, 2013; Wu et al., 2009).

To date, it is not clear if the different modes of endocytosis - in neuronal cells, these are clathrinmediated endocytosis (CME), activity-dependent bulk endocytosis (ADBE), ultrafast endocytosis (UFE) and potentially "kiss-and-run" (seeFig. 1-3) - are initiated via different triggers. There is some evidence that particularly changes of membrane tension induce UFE (see 1.3.2.3 Ultrafast Endocytosis; W atanabe et al., 2013; W atanabe et al., 2018). It is likely that also the prompt clearance of release sites, which seems to be a prerequisite for sustained SV release, serves as an initiator for UFE (Gan and W atanabe, 2018; M aritzen and H aucke, 2018; N eher, 2010; W atanabe et al., 2013). However, the full retrieval of SV cargo proteins does not appear to be obligatory for sustained exocytosis due to the availability of cytosolic SV pools. Thus, the sorting of membrane-stranded SV cargo is rather a trigger for the slower CME (Denker et al., 2011; Gan and W atanabe, 2018; Rizzoli and Betz, 2005). 


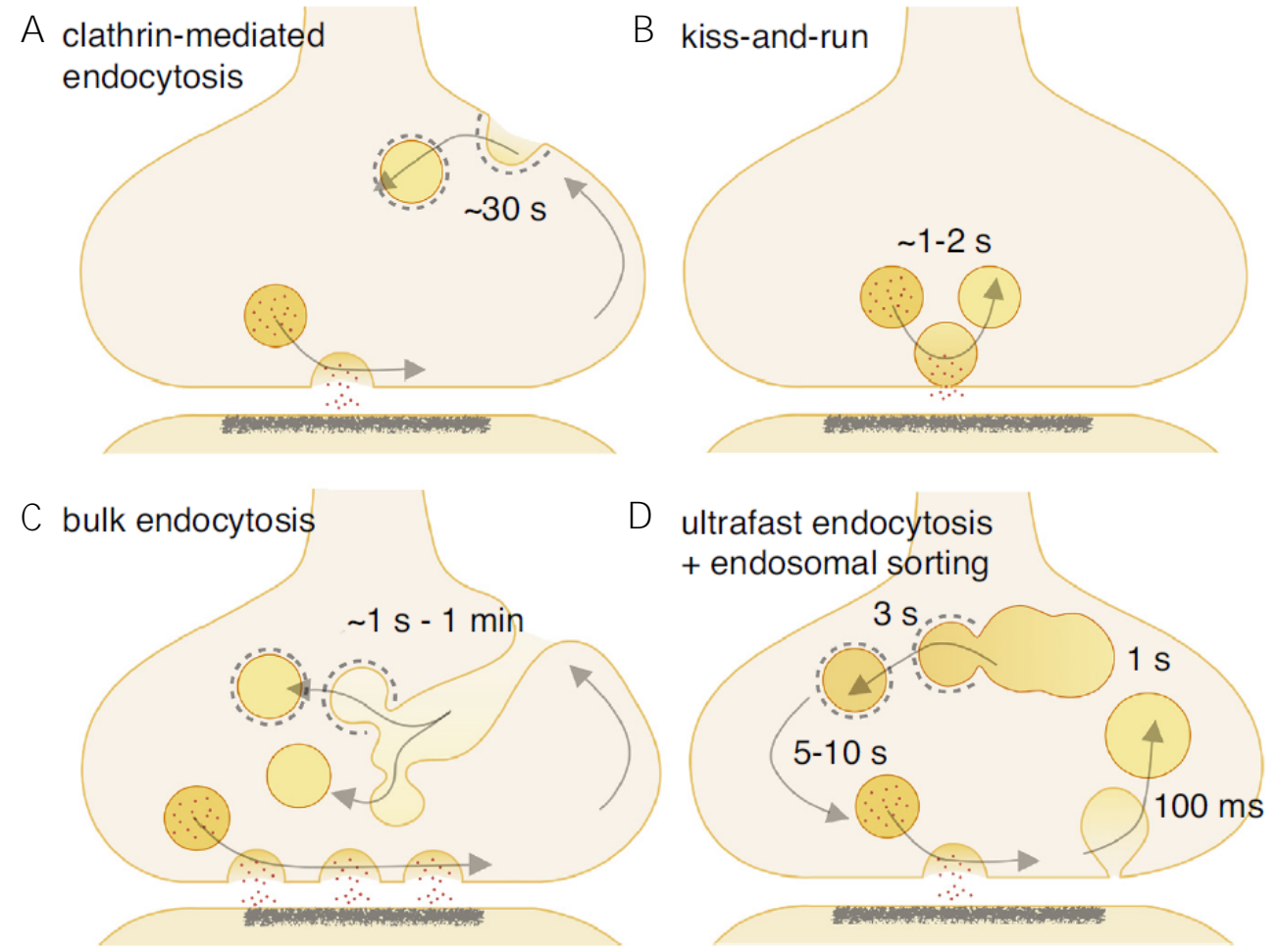

Fig. 1-3: Endocytic mechanisms at synapses. (A) CME is the most-studied form of endocytosis and is defined by the formation of clathrin-coated pits in the peri-active zone (in neurons), which are subsequently fissioned and uncoated. (B) "Kiss-and-run" is characterized by the opening and closing of a fusion pore without full integration into the AZ membrane. (C) ADBE is induced by strong stimulation. Large membrane compartments are internalized, of which SVs are reformed via clathrin-dependent and/or independent mechanisms. (D) UFE has been observed less than $100 \mathrm{~ms}$ after stimulation. In direct vicinity of the $A Z$, endocytic vesicles are internalized. These fuse with endosomes, followed by clathrin-dependent SV reformation. Illustration from (Watanabe and Boucrot, 2017).

It has further been shown that intensity and duration of a stimulus as well as temperature play a role in the induction of different endocytic mechanisms. Few action potentials at physiological temperature were shown to be sufficient to trigger UFE in neurons (W atanabe et al., 2013). Already at room temperature and upon low to moderate stimulation, CME has been ascertained in neurons (Granseth et al., 2006; Kononenko et al., 2014; Saheki and Camilli, 2012), as well as in IHCs (N eef et al., 2014; Siegel and Brownell, 1986). Additionally, stimulation with a high frequency or with high$\mathrm{K}^{+}$was shown to induce the clathrin-independent internalization of bulk membrane, from which SV s are subsequently reformed (Clayton and Cousin, 2009; Clayton et al., 2008; Kantardzhieva et al., 2013; N eef et al., 2014; Revelo et al., 2014). Particularly at synapses with very fast and continuous SV turnover - not only in IHC and retinal ribbon synapses (M oser and Beutner, 2000; N eef et al., 2014; 
Paillart et al., 2003) but also at central synapses like the calyx of Held (Clayton et al., 2008; Lange et al., 2003; W u and Wu, 2007) - bulk-like membrane retrieval (e.g. ADBE) has been observed.

For more than three decades, CME has broadly been accepted as the predominant mode of endocytosis in neurons and sensory cells (Granseth et al., 2006; Saheki and Camilli, 2012). Recent studies propose that clathrin-independent pathways have a major role in endocytosis whereas CME is more important for cargo sorting than for membrane retrieval (Gimber et al., 2015; Kononenko et al., 2014; Soykan et al., 2017; W atanabeet al., 2013). In these studies, theauthors argue that knockouts of clathrin adaptor proteins like AP-2 (Gu et al., 2008; Jung et al., 2015b; Kononenko et al., 2014), stonin2 (Kononenko et al., 2013), and AP180 (Koo et al., 2015) as well as absence or inhibition of clathrin heavy chain ( $N$ eef et al., 2014; Sato et al., 2009) only mildly impair the kinetics of membrane retrieval but result in a greater diffusional spread of proteins that were undergoing exocytic processes (Gimber et al., 2015; Soykan et al., 2017). H owever, absence of clathrin or its adaptors also resulted in accumulations of ELVs, which are interpreted as endocytic intermediates. Therefore, it was proposed that the reformation of SV s from ELVs following ADBE or UFE in neurons as well as in IHCS is at least in parts dependent on clathrin and on AP-2 (Jung et al., 2015b; Kononenko et al., 2014; W atanabe et al., 2014). Interestingly, mechanisms and protein machinery involved in "classical CME" and in clathrin-dependent SV reformation seem to be at least in part identical (Gan and W atanabe, 2018). It has even been hypothesized that CME and clathrin-dependent SV-reformation are in principle the same mechanism, only that strong stimulation induces bulk endocytosis and concomitantly results in an increase in accessible surface area, so that adaptors can more easily recruit clathrin (W atanabe and Boucrot, 2017).

\subsubsection{CLATHRIN-DEPENDENT SV RECYCLING}

Even though future studies will be required to exhibit the physiological relevance of $\mathrm{CME}$, molecular mechanisms of clathrin-coated pit (CCP) formation, fission and uncoating have extensively been examined in neurons. Whether CME is executed in the same molecular manner in IHCs is not clear yet. Neuronal CME and clathrin-dependent SV reformation are initiated by the binding of adaptor proteins to SV cargo (Edeling et al., 2006; Kononenko et al., 2014). Binding sites for clathrin, phospholipids like $\mathrm{PI}(4,5) \mathrm{P}_{2}$, and SV cargo facilitate the recruitment of clathrin light chain and clathrin heavy chain triskelia to the periactive zone, where CME in neurons is supposed to take place, as well as to ELVs (Ford et al., 2001; Koo et al., 2011). Self-polymerization of clathrin around the 
nucleation site results in the assembly of a distinctive lattice, the clathrin coat (Kirchhausen, 2000). The clathrin coat-induced membrane curvature is stabilized and intensified by curvature-sensing proteins like endophilin-A, resulting in a deeply-invaginated CCP connected to the membrane via a neck (Farsad et al., 2001; Gallop et al., 2006, 2006). Endophilin-A , henceforth endophilin, coordinates further steps of CME via recruitment of the GTPase dynamin and the phosphatase synaptojanin-1 (Anggono and Robinson, 2007; Verstreken et al., 2003). When located at the neck of the CCP, dynamin polymerizes and thus induces fission of the pit (Ferguson et al., 2007). The now cytosolic, unbound clathrin-coated vesicle (CCV) has to be uncoated. Synaptojanin-1 hydrolyzes $\mathrm{PI}(4,5) \mathrm{P}_{2}$ and thus causes dissociation of clathrin adaptors bound to the phospholipid (Cremona et al., 1999; Pechstein et al., 2015; Schuske et al., 2003; V erstreken et al., 2003). TheATPase H sc70 and its cofactor auxilin further support the disassembly of the clathrin coat (Guan et al., 2010; Xing et al., 2010).

M embrane capacitance $\left(C_{m}\right)$ recordings in IHCs revealed that brief depolarizations activate a slow form of endocytosis with a linear return to baseline (Neef et al., 2014). Both, the clathrin-inhibitor pitstop- 2 and the pharmacological and genetic disruption of the GTPase dynamin 1 were shown to further slow this already slow mode of endocytosis, which has thus been associated with CME (N eef et al., 2014). A bsence of theadaptor protein A P-2 did not result in significantly impaired endocytosis but in impaired clathrin-dependent SV reformation (Jung et al., 2015b). Therefore, it is not clear yet if CME plays a minor role in IHCs or if the absence of AP-2 could e.g. be partially compensated by other clathrin-recruiting adaptor proteins like AP-1, AP-3, or AP180 at least for the formation of CCPs at the plasma membrane. A part from clathrin, dynamin, AP-2, and amphiphysin (as well as synaptojanin-1, which was shown to play a role in IHC endocytosis in zebrafish), knowledge on the molecular entities mediating SV recycling in IHCs remains sparse (Boumil et al., 2010; Duncker et al., 2013; Jung et al., 2015b; N eef et al., 2014; Siegel and Brownell, 1986; Trapani et al., 2009).

Controversial hypotheses have been postulated regarding the further steps an uncoated vesicle has to undergo in order to become fusion-ready. Observations of endosomal structures with budding CCPS in the cytosol led to the original hypothesis that all CCV s fuse with endosomes, which serve as sorting stations for SV proteins (Heuser and Reese, 1973). However, it could be shown that CCV s are first uncoated (Rothman and Schmid, 1986), which clearly speaks against theinitial hypothesis of Heuser and Reese. Instead, it was suggested that uncoated vesicles directly undergo new rounds of exo- and endocytosis (Takei, 1996). M ore recent studies showed that endosomes are indeed involved in the sorting of SV cargo and potentially of SV membranes, as e.g. endosomal SNARE proteins have been 
identified on the surface of SV s (Jähne et al., 2015, 201; Rizzoli et al., 2006). H owever, it is not clear if fusion of uncoated vesicles to endosomes is a common process following $\mathrm{CM}$ E/ clathrin-dependent SV reformation, or if this pathway is predominantly involved in the degradation of vesicles that contain wrong, old, or damaged proteins (Jähne et al., 2015; Truckenbrodt et al., 2018). In any case, the uncoated vesicles following CME seem to fuse with bona fide endosomes rather than with endocytic intermediates observed during ADBE and during UFE (Jähne et al., 2015). Whether SV maturation in neurons and sensory hair cells follows similar pathways, is not known.

\subsubsection{ACTIVITY-DEPENDENT BULK ENDOCYTOSIS}

Few seconds up to minutes after intense stimulation, ELVs, probably originating from bulk endocytosis, have been observed in the cytosol of neurons as well as of IHCs. These ELVs are heterogeneous in size, probably correlating with the amount of exocytosed membrane material (Clayton and Cousin, 2009; Kamin et al., 2014; N eef et al., 2014; Revelo et al., 2014; W atanabe and Boucrot, 2017). Experiments using the membrane marker mCLING or FM-dyes in combination with photo-oxidation and subsequent electron microscopy imaging, both after high- $\mathrm{K}^{+}$stimulation, indicated that SV s are subsequently reformed from the internalized ELV s (Kamin et al., 2014; Revelo et al., 2014). Electrophysiological recordings revealed that harsh stimulation, exceeding three to four RRP equivalents, induces a brief, exponential $C_{m}$ decline in IH Cs that likely reflects ADBE (Beutner et al., 2001; Moser and Beutner, 2000; Neef et al., 2014). Even though bulk-like membrane internalization was shown to be almost exclusively performed at the IHC base and in particular in close proximity to the ribbon synapses, a distinct area of endocytosis could not be identified yet in those cells (Kantardzhieva et al., 2013; Revelo et al., 2014; Siegel and Brownell, 1986; W ichmann and M oser, 2015). In contrast, photoreceptor ribbon synapses clearly exhibit a periactive zone, as seen by a the high prevalence of endocytic proteins in proximity to the AZ (W ahl et al., 2013).

Not only increased membrane tension but also $\mathrm{Ca}^{2+}$-influx seem to induce ADBE at room temperature as well as at physiological conditions (Wu et al., 2009). Even though the mechanisms involved in ADBE are not fully understood yet, recent studies have propagated a model in which assembly of actin organized by formins is crucial for activity-driven membrane invaginations in neurons (Soykan et al., 2017). It is not clear if membrane fission is mediated by dynamins (Soykan et al., 2017), works independent of dynamin (W u et al., 2014), or if different dynamin-dependent and -independent pathways are present e.g. at different synapses (Gan and W atanabe, 2018). 
At this point, it has to be mentioned that the physiological relevance of ADBE in hippocampal neurons has been under discussion (Gan and W atanabe, 2018; Kononenko and Haucke, 2015) for several reasons: (i) freshly recycled SV s are preferentially used for exocytosis, SV s in the reserve pool are significantly older (Truckenbrodt et al., 2018), going along with the observation that (ii) the reserve pool is not mobilized under physiological conditions but activated during very intense stimulation due to depletion of recycling pools (Rizzoli and Betz, 2005), and (iii) that ELVs formed during ADBE are randomly sized and retrieved in a rather uncoordinated manner ( $G$ an and Watanabe, 2018). Therefore, ADBE may display an emergency pathway in which the cell tries to counteract the drastic increase in cell membrane accompanied by accumulations of exocytosisrelated proteins (Gan and W atanabe, 2018). However, particularly synapses with extremely fast and continuous SV turnover, e.g. IHC ribbon synapses as well as synapses at the Calyx of Held, may require bulk-like endocytosis mechanisms rather than UFE to keep SV pool sizes stable and thus synapse function viable.

\subsubsection{ULTRAFAST ENDOCYTOSIS}

In contrast to ADBE, UFE seems not suitable for compensation of high endocytosis rates over longer time periods, as the capacity of this process is limited to few action potentials (Soykan et al., 2017; W atanabe et al., 2013). UFE was originally described by Watanabe et al., 2013 in a novel approach combining flash and freeze(light stimulation of channelrhodopsin-expressing hippocampal neurons at physiological temperatures followed by HPF) with high-resolution electron tomography. The authors could show that endocytic vesicles with a diameter of $60-80 \mathrm{~nm}$ are formed within 30-50 ms in hippocampal neurons (W atanabe et al., 2013; W atanabe et al., 2018). This process seems to carry on for roughly one second and takes place in direct vicinity of the active zone, whereas neuronal CM E and ADBE aretypically detected in the periactivezone (W atanabeet al., 2013). W hether limited diffusion of exocytic proteins due to the very short time span or a specialized protein/ lipid composition of the AZ borders are responsible for the untypical localization of this endocytic event is not clear yet (Gimber et al., 2015; W atanabe and Boucrot, 2017). The volume of one endocytic vesicle matches approximately four SVs, the total volume of the UFE-mediated membrane internalization is equal to the surface extension following few action potentials, which indicates a direct compensatory effect of UFE (W atanabe et al., 2013). This hypothesis is supported by the fact 
that $\mathrm{Ca}^{2+}$-influx without fusion events cannot trigger UFE, as seen in exocytosis-deficient $\mathrm{M}$ unc13 mutants (W atanabe et al., 2013).

The formation of membrane invagination requires filamentous actin; either for the maintenance of surface tension while other factors initiate membrane bending, or via active polymerization (W atanabe and Boucrot, 2017; W atanabe et al., 2013). Once the endocytic pit is formed, endophilin and synaptojanin-1 facilitate the constriction of the neck, which is eventually cleaved by dynamin (Watanabe et al., 2013; Watanabe et al., 2018). However, absence of endophilin and/ or synaptojanin-1 does not block scission of the endocytic pit but rather slows down this process (W atanabe et al., 2018). The endocytic vesicles fuse with endosomes, which may be required for protein sorting, followed by subsequent clathrin-dependent SV reformation (Kononenko et al., 2014; W atanabe et al., 2014). The whole process from invagination to SV reformation takes roughly 10 20 seconds and is suggested to display the predominant form of endocytosis in neurons under physiological conditions (W atanabe and Boucrot, 2017; W atanabe et al., 2014). In IHCs, UFE has not been observed yet.

\subsubsection{ENDOPHILIN-A ANd AP180 - Key PLAYERS In SyNAPTIC TRANSMISSION}

As al ready described in the previous chapters, dynamin plays a key role in fission of CCPs from the plasma membrane and from ELVs, as well as in the cleavage of endocytic vesicles during ultrafast endocytosis. Dynamin contains a pleckstrin homology domain that is involved in phospholipid binding. Oligomerization of the GTPase dynamin at the neck of an endocytic or coated pit results in energy-releasing GTP hydrolysis und ultimately in membrane cleavage (H inshaw, 2000). Through its SRC homology 3 (SH3) domain, dynamin is recruited by e.g. endophilin, whereby endophilin absence does not result in a complete block of dynamin function but rather in slowed kinetics (M ilosevic et al., 2011; W atanabe et al., 2018). Recent studies suggest that endophilin, when present in excess, can inhibit dynamin action, pointing towards a regulating function of endophilin in endocytic fission (Hohendahl et al., 2017). 


\subsubsection{THE KEY REgULATOR ENDOPHILIN-A}

ENDOPHILINS BAR AND SH3 DOMAINS ARE REQUIRED DIVERSE Functions

Through interactions with a large number of proteins predominantly involved in endocytic processes, endophilin is seen as a hub that orchestrates the induction and stabilization of membrane curvature, bud constriction, fission, and uncoating in neurons (see Fig. 1-4B; Saheki and Camilli, 2012). In vertebrates, three members of the endophilin-A family have been identified, of which endophilin-A1 is brain specific, endophilin-A2 is ubiquitously expressed, and endophilin-A 3 is enriched in brain and testis (Giachino et al., 1997; Ringstad et al., 1997). The three endophilin genes are highly similar, which allows for partial compensation if one or more endophilin alleles are absent ( see Fig. 1-4A; M ilosevic et al., 2011; M urdoch et al., 2016).

In general, endophilins contain an N-terminal BAR (Bin-Amphiphysin-Rvs) domain and a Cterminal SH 3 domain and form crescent-shaped homodimers (Gallop et al., 2006; Ringstad et al., 1997). The BAR domain of endophilin was shown to induce membrane curvature, to stabilize existing membrane invaginations, and to sense membrane curvature in order to recruit further proteins to the neck of CCPs (Farsad et al., 2001; Gallop et al., 2006; M asuda et al., 2006). Recent studies suggest that the organized recruitment of endophilin to the nucleation sites in neurons is facilitated by the multi-domain scaffolding protein intersectin- 1 via interaction of their SH 3 domains (Pechstein et al., 2015). The SH 3 domain of endophilin further interacts with the proline-rich domains of dynamin (Ferguson et al., 2009; Ringstad et al., 1997) and of synaptojanin-1 (Perera et al., 2006; Schuske et al., 2003; V erstreken et al., 2003). 
A

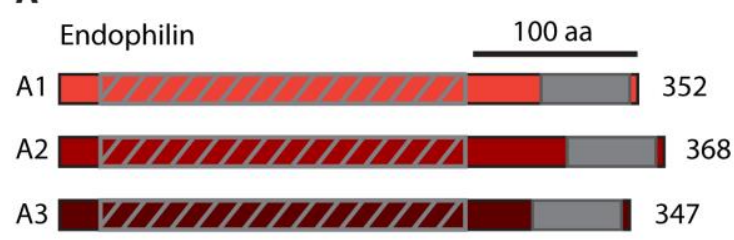

$\square \mathrm{N}-\mathrm{BAR}$ domain

$\square$ SH-3 domain
B

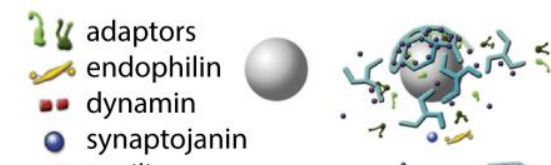

- synaptojanin

$\therefore$ auxilin

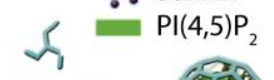

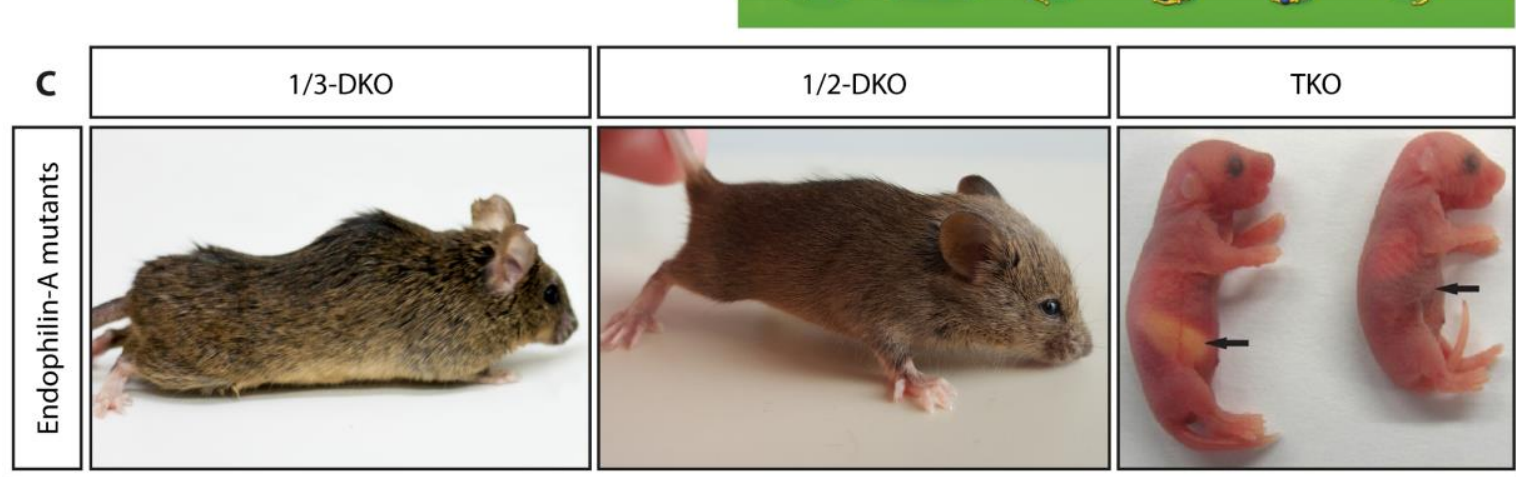

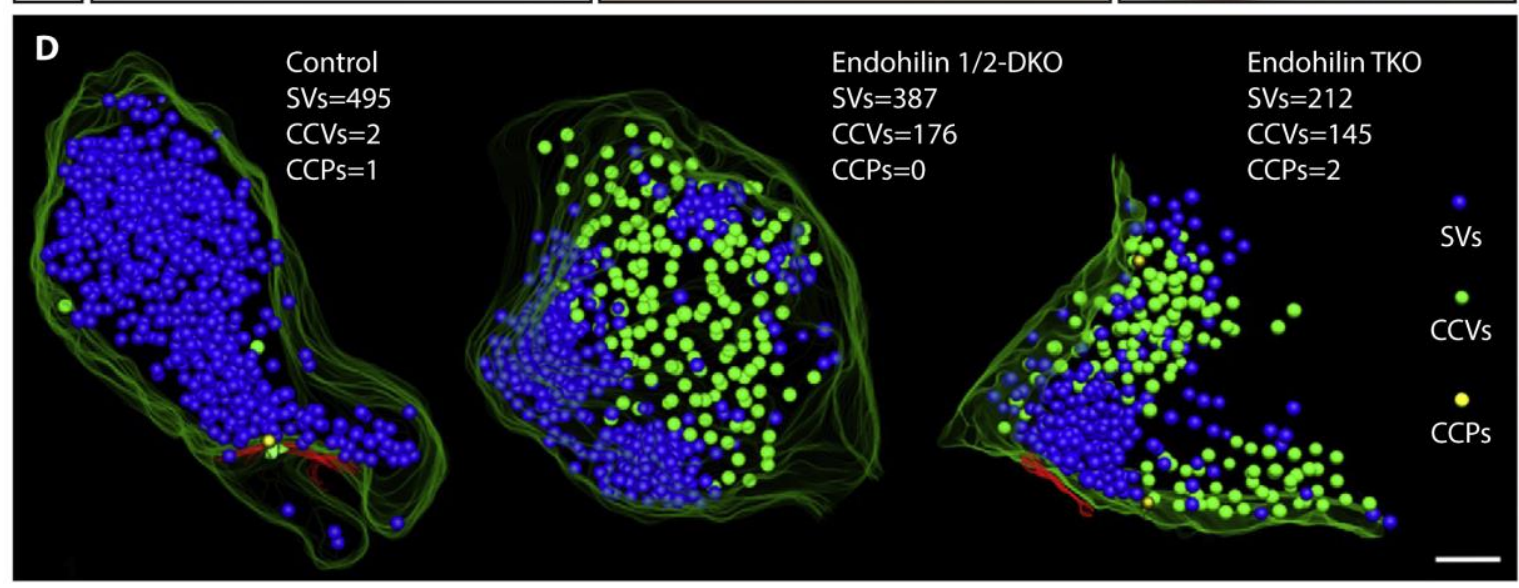

Fig. 1-4: Absence of endophilin-A results in disturbed SV recycling. (A) The three mammalian endophilin-As show a high similarity. All three contain a BAR domain and an SH3 domain. (B) Endophilin-A is involved in different steps of CME via regulating the fission and uncoating process. (C) Endophilin 1/3DKOs show increasing motor defects with age, 1/2-DKOs have a truncated life expectancy paired with major neurological and motor defects. TKOs survive few hours after birth. (D) Absence of endophilins leads to accumulations of CCVs and reduced numbers in SVs in 1/2-DKOs and even more prominently in TKOs. Images modified from (Milosevic et al., 2011; Murdoch et al., 2016).

\section{KNOCKOUT MODELS FOR ENDOPHILIN}

Even though endophilin is active in different steps of CME/ clathrin-dependent SV reformation, particularly the uncoating process seems to be of physiological relevance, as studied in diverseanimal models: Genetic studies in Drosophilia, C. elegans and mice revealed accumulations of CCVs accompanied by impaired synaptic transmission in absence of endophilins (see Fig. 1-4D; Dickman 
et al., 2005; M ilosevic et al., 2011; Schuske et al., 2003; Verstreken et al., 2003). A similar phenotype has been described for synaptojanin-1 KO models (Cremona et al., 1999; M ilosevic et al., 2011).

A bsence of all three endophilins in mice (see Fig. 1-4C; triple-knockouts; TKOs) was shown result in a life expectancy of only few hours after birth, likely induced by impaired synaptic transmission, breathing problems, and the inability to drink milk (M ilosevic et al., 2011). Hippocampal neurons of TKOs exhibit accumulations of CCV s, whereas numbers of uncoated SV s are significantly decreased (M ilosevic et al., 2011). Electrophysiological recordings in those TKO cells further indicated reduced rates of sustained exocytosis, which may be a result of impaired SV recycling or indicate a potential involvement of endophilin in SV fusion and/ or SV replenishment (Milosevic et al., 2011). Double knockouts lacking endophilin A1 and A2 (1/2-DKOs) have a drastically truncated life expectancy of only 2-3 weeks, a strong growth delay, as well as major neurological and motor impairments (Milosevic et al., 2011). Mutants missing endophilin A1 and A3 (1/3-DKOs) display motor impairments and epileptic seizures with age-dependent increasing severity (M ilosevic et al., 2011; Murdoch et al., 2016). Additional heterozygous deletion of endophilin A2 further increases these symptoms. Eventually, these observations have been linked to increased apoptosis rates in neurons (M ilosevic et al., 2011; M urdoch et al., 2016). Whether absence of endophilins results in hearing defects and impaired endocytosis in IHCs has been investigated in the first chapter of my studies.

\section{FUnCTIONS OF ENDOPHILIN BEYOND ENDOCYTOSIS}

It has been ascertained that not only defective endocytosis, but also disturbed protein homeostasis via upregulation of the cellular protein degradation system (autophagy) are responsible for neurodegenerative processes in endophilin mutants (M urdoch et al., 2016). This finding goes along with previous studies, in which interactions of endophilin with proteins involved in Parkinson's disease, namely parkin and LRRK2, have been identified (Cao et al., 2014; Soukup et al., 2016).

Endophilin has furthermore been shown to interact with voltage-gated $\mathrm{Ca}^{2+}$-channels ( $\mathrm{Chen}$ et al., 2003). Performing co-immunoprecipitations and pull-downs, the authors detected a $\mathrm{Ca}^{2+}$-dependent formation of complexes formed by endophilin and $\mathrm{Ca}^{2+}$-channels. Therefore, they concluded that endophilin is involved in the coupling of exo- and endocytosis (Chen et al., 2003).

Lastly, we could show in work not included in this thesis that endophilin is involved in the recruitment, priming, and fusion of large dense core vesicles (LDCVs) in chromaffin cells 
(Gowrisankaran et al., unpublished). Theseneuroendocrine cells are a favored model system to study neurosecretion, as key players in $\mathrm{Ca}^{2+}$-induced neurotransmitter release are identical in chromaffin cells and in neurons (De Camilli and Jahn, 1990; Neher, 2006). We could show that exocytosis in endophilin TKOs is reduced while numbers of LDCVs and cell size as well as SV recycling are unaffected. However, distances of LDCVs to the cell membrane, where release takes place, were found to beincreased in adrenal gland tissue of TKO spointing towards deficits in SV replenishment. In a multi-methodological approach, we could show that this novel role of endophilin in LDCV recruitment and release is, at least in parts, achieved by the interaction of endophilin and intersectin.

\subsubsection{THEAdAPTOR ProteIn AP180}

\section{AP180 IS REQUIRED FOR CLATHRIN RECRUITMENT AND VAMP2 SORTING}

While endophilin is active in multiple steps of clathrin-mediated membrane retrieval, adaptor proteins have a major role in the recruitment and assembly of clathrin molecules to the nucleation sites. The brain-enriched AP180 and its ubiquitously expressed homolog CALM both contain an ANTH (AP180 N-terminal homology) domain and an unstructured sequence with multiplebinding motifs for clathrin and A P-2 (see Fig. 1-5A; H ao et al., 1999; Lindner and U ngewickell, 1992; M orris et al., 1993; M oshkanbaryans et al., 2016). The C-terminus differs in length and binding sites for endocytic proteins, whereas the ANTH domain is highly conserved between AP180 and CALM (Maritzen et al., 2012). This domain harbors an $\alpha$-helical structure capable of binding lipids and therefore serves as connection to the $\mathrm{PI}(4,5) \mathrm{P}_{2}$-containing plasma membrane (Ford et al., 2001). Furthermore, AP180 and CA LM act in the sorting of the SNARE protein VAM P2, as seen in neurons with AP180 depletion, where VAMP2 is stranded at the plasma membrane (Koo et al., 2011). Interestingly, in contrast to many other interactions between cargo proteins and their adaptors, the SNARE motif of VAMP2 itself binds to the ANTH domain of AP180 and CALM (Koo et al., 2011; Miller et al., 2011). Also other proteins of the VAMP family like VAMP3 and VAM P8, which are involved in endosomal sorting, are capable of binding to the ANTH domain (M aritzen et al., 2012; Miller et al., 2011). In any case, the disassembly of the trans-SNARE complex must precede the binding of AP180/CALM, as both events utilize the same binding site of the VAM Ps (see Fig. 1-5C; Miller et al., 2011). Therefore, AP180 and CALM may also have a function in preventing VAM Ps from undergoing unwanted SNARE complex formations, e.g. directly after fusion (Kaempf and M aritzen, 2017). 
A

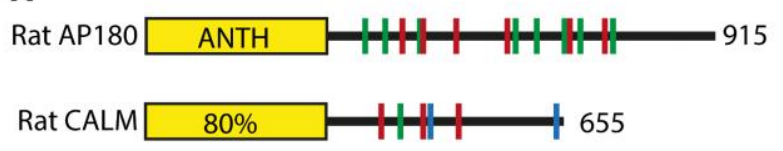

C

\section{(1)}

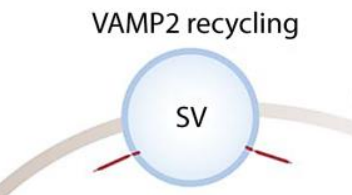

I Clathrin-binding motifs

I EH domain-binding motifs

I AP2-binding motifs
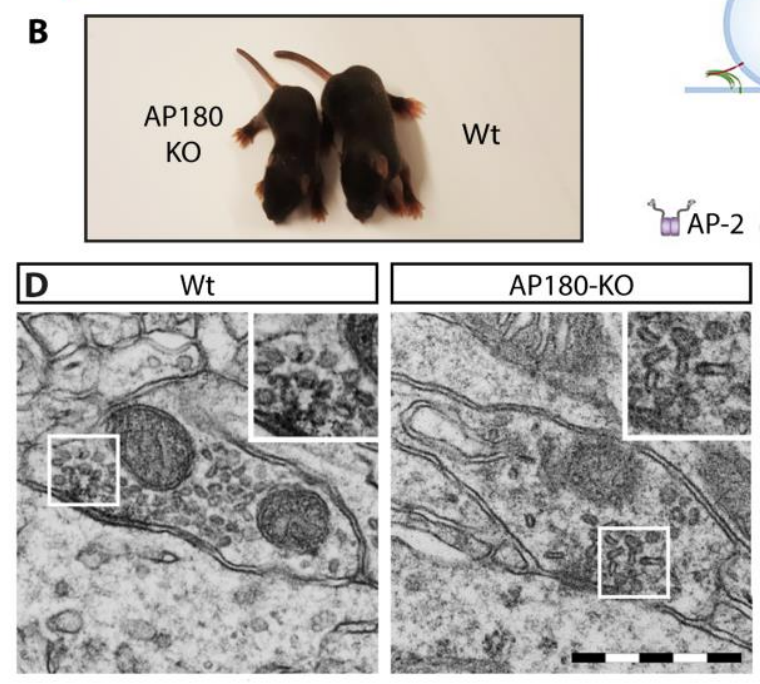

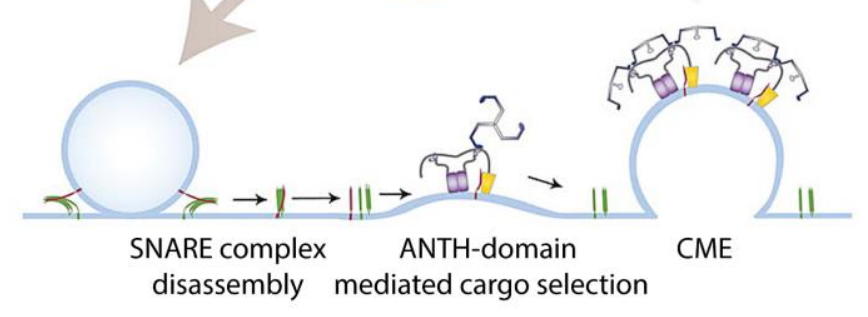

"zrAP-2 APAP180/CALM 己Clathrin IVAMP2!Syntaxin1 ISNAP25 E
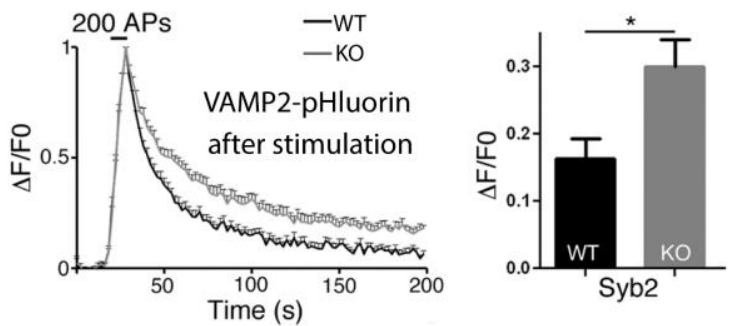

Fig. 1-5: AP180 is involved in neuronal SV reformation and release site clearance. (A) AP180 and CALM contain a highly conserved ANTH domain involved in VAMP2 binding. Within the unstructured C-terminal domain, both proteins contain binding sites for clathrin and AP-2. (B) AP180-KO mice have a truncated life expectancy of max. 1-2 months paired with severe behavioral abnormalities including epileptic seizures. (C) AP180 is involved in clathrin-dependent SV reformation and in the sorting of VAMP2 post-fusion. (D) Absence of AP180 leads to reduced numbers of SVs particularly in synapses of inhibitory hippocampal neurons. Scale bar $500 \mathrm{~nm}$. (E) Fluorescence intensity of VAMP2-pHluorin over a time course of $200 \mathrm{~ms}$ after stimulation indicates that unretrieved VAMP2 (but no other SV proteins) was found to be stranded at the surface of cultured hippocampal neurons from AP180-KO mice. Images modified from (Koo et al., 2015; Maritzen et al., 2012).

\section{KNOCKOUT MODEL FOR AP180}

Despite evidence that sorting of VAM Ps depends on AP180, the physiological relevance of AP180 for synaptic transmission has been questioned. The main reason is, that the formation of only few SN ARE complexes is essential for SV fusion whereas roughly 70 V AM P2 proteins are located at one SV (M ohrmann et al., 2010; Sinha et al., 2011; Takamori et al., 2006; van den Bogaart et al., 2010; W ilhelm et al., 2014). Studies on A P180-KO mice revealed that A P180 does indeed play a crucial role in vivo, as those mutants suffer from a growth delay, a truncated life expectancy of only few months or even less, as well as hyperactivity and epileptic seizures (see Fig. 1-5B; Koo et al., 2015). Particularly 
in inhibitory neurons, which are supposed to be more persistently active than excitatory neurons, sustained neurotransmission was impaired. The synaptic rundown in response to repeated stimulation has been explained by impaired A P180-dependent SV reformation as well as by disturbed SV replenishment due to reduced copy numbers of VAMP2 at SVs and the SNARE protein's role in docking/priming (Koo et al., 2015). Impaired release site clearance, as ascertained by membranestranded VAM P2 accumulations in AP180-KO mice (see Fig. 1-5E), may additionally contribute to the observed defects in exocytosis following repeated stimulation (Gimber et al., 2015; Kaempf and M aritzen, 2017; N eher, 2010). N otably, even though CALM was upregulated in AP180-KO mice, this protein could not fully compensate for the loss of AP180 (Koo et al., 2015). Whether IH Cs, which show remarkably high rates of SV turnover and continuous neurotransmitter release, but operate without VAM P2, require A P180, will be investigated in the second chapter of my studies. 


\subsection{AIMS AND EXPERIMENTAL APPROACH}

\subsubsection{AIMS OF MY STUDY}

In the past, proteins and mechanisms involved in IHC exocytosis have extensively been examined. H owever, knowledge about SV recycling in these highly adapted sensory cells remained sparse. Few studies have shown that endocytic mechanisms in IHC s are activity-dependent and contain clathrindependent as well as clathrin-independent pathways (Jung et al., 2015b; Kamin et al., 2014; M oser and Beutner, 2000; Neef et al., 2014; Revelo et al., 2014). Yet, molecular key players in IHC endocytosis are largely unknown. Neither do we currently understand, how exo- and endocytosis are balanced and coupled in IHCS, although deficits in SV replenishment were shown to go along with severe hearing impairments (Pangršič et al., 2010; Strenzke et al., 2016). Studies in CNS synapses have not only focused on the characterization of endocytic proteins mediating different steps and mechanisms of SV recycling; they also demonstrated that proteins originally identified as endocytic factors are involved in release site clearance, protein sorting and coupling of different steps within theSV cycle (for review see M aritzen and H aucke, 2018). Endophilin-A has originally been described as hub orchestrating different steps of CME with a rate-limiting function in the uncoating of CCVs (Milosevic et al., 2011). Recently, endophilin-A was additionally shown to act in clathrinindependent endocytosis via regulating the fission of endocytic vesicles during UFE (W atanabe et al., 2018). The adaptor protein AP180 is not only required for the recruitment of clathrin, but has also a function in release site clearance via sorting of VAM P2 (Koo et al., 2015).

In the here presented two studies, I combined a set of advanced imaging techniques to characterize morphological changes in mouse mutants missing different endophilin-A genes or AP180. This way, I coul examine the distinct roles of these two proteins/ protein families in IHCs with a focus on their functions in SV recycling. Combining the two studies, I could compare different endocytic steps being dependent on either of the two proteins. This way, I gained deeper insights into the relevance of the different endocytic mechanisms for IHC synaptic transmission.

\subsubsection{EXPERIMENTAL APPROACH}

In the first study presented, I examined a set of mutants lacking different endophilin genes. In detail, I compared Wt mice with mutants missing endophilin-A1 and -A2 (1/2-DKOs) as well as their littermates missing only endophilin-A1 (1-SKOs), all with the same mixed genetic background 
(approx. 80\% BI6/J and 20\% S-129). M oreover, I analyzed endophilin A 1 and A 3 mutants that were heterozygous or wildtype for endophilin A2. Since neither microscopical nor physiological analyses revealed any differences in IHCs if endophilin A2 was heterozygously expressed, we pooled those two mutants as one group of 1/3-DKOs. I used all of these mutants to perform electron microscopy experiments. Due to our interest in the general organization of the $A Z$ including endocytic intermediates, and to guarantee for comparability with a previous study on AP- $2 \mu$ in IHCs (Jung et al., 2015b), I performed conventional EM embeddings including chemical fixation, partially with preceding 15 min high- $\mathrm{K}^{+}$stimulation. Like in the AP- $2 \mu$ study, I mainly performed my analyses on images from random ultrathin sections acquired with 12,000 x magnification (for analysis of RA-SV and MP-SV numbers) and with 5,000 x magnification (to analyze the ribbon proximity within $1 \mu \mathrm{m}$ radius from the ribbon) on a JEO L JEM 1011 electron microscope. I additionally performed electron tomography on 1/2-DKO sand 1/3-DKO sin comparison to $\mathrm{Wt}$. Only with the 3D information of the acquired tomograms I could make sure that neither "clathrin-trees", that have previously been observed in absence of dynamins (Ferguson et al., 2007; Ferguson et al., 2009), nor malformed SV s (as e.g. detected in mutants harboring a point mutation within the otoferlin gene (Strenzke et al., 2016)) were present in the endophilin mutants. M oreover, I could reliably analyze the diameter of SVs. Nonetheless, even with the increased resolution and 3D information of tomograms, examinations on ELVs, which can expand for more than $250 \mathrm{~nm}$ in each direction (thickness of sections for electron tomography used here), are difficult to interpret. I could for example not always clearly state if an ELV is connected to the plasma membrane or not. Neither was it possible to differentiate between newly formed ELV s and bona fide endosomes based on their morphology. Therefore, I categorized all large membranous organelles without clathrin-coat as ELVs in my quantifications. I combined my comprehensive electron microscopy analyses with confocal microscopy. Since we observed impairments in the sustained phase of exocytosis in 1/3-DKOs, we were curious if these changes are caused by reduced otoferlin levels. To test this, I used wellestablished immunostaining protocols and analysis routines (Strenzke et al., 2016). Moreover, I analyzed the area of $\mathrm{Ca}^{2+}$-channel clusters beneath the ribbon from 2D STED microscopy images as previously described for I HC ribbon synapses (J ean et al., 2018; Krinner et al., 2017; N eef et al., 2018), since we observed slightly decreased $\mathrm{Ca}^{2+}$-currents in several endophilin mutants. M y collaborators performed electrophysiology, biochemistry and systems physiology experiments to generate a wholesome characterization of the IHC phenotype in absence of the different endophilins. 
For my second study, I performed HPF and freeze substitution combined with electron tomography in Wt and AP180-KO mice. It is commonly accepted, that this method allows for a near-to-native state structural preservation of filamentous and membranous structures. Yet, identifying clathrincoated structures in high-pressure frozen samples is more challenging than in conventionally embedded tissue. We used HPF despite AP180's potential role in clathrin recruitment since AP180 was also shown to be involved in release site clearance in neurons (Koo et al., 2015) and wewere thus particularly interested in the AZ membrane and tethered SV s. The combination of HPF and electron tomography allows for a thorough analysis of tethers connecting SV s. V ariations in tether numbers and tether lengths have previously been studied to morphologically characterize potential defects upor downstream of docking (Chakrabarti et al., 2018; Vogl et al., 2015). Furthermore, electron tomography allowed meto more reliably quantify ELV volumes and numbers of budding CCPs, both indicators for clathrin-dependent SV reformation. I additionally performed immunohistochemical stainings following standard protocols and using well-established IHC markers. Together, my morphological studies gave new insights also into the potential functions of AP180 in IHCS. 


\section{ChAPTER ONE}


Chapter One | 


\subsection{GENERAL INFORMATION}

TITLE: ENDOPHILIN-A REGULATES PRESYNAPTIC CALCIUM INFLUX AND SYNAPTIC VESICLE RECYCLING IN AUDITORY HAIR CELLS

ABBREVIATED TITLE: ENDOPHILINS MODULATE HAIR CELL SYNAPSES

AUTHORS:

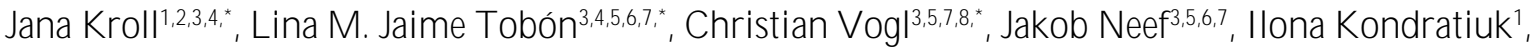
Melanie König ${ }^{1,3}$, Nicola Strenzke ${ }^{3,7,9}$, Carolin Wichmann ${ }^{2,3,7, \#,}$ Ira Milosevic ${ }^{1,3, *}$ and Tobias Moser $3,5,6,7, \#$

'Synaptic V esicle Dynamics Group, European Neuroscience Institute Göttingen - A Joint Iniciative of the University M edical Center Göttingen and the M ax-Planck-Society, Göttingen, Germany

${ }^{2} \mathrm{M}$ olecular A rchitecture of Synapses Group, Institute for Auditory N euroscience, InnerEarLab and Center for Biostructural Imaging of Neurodegeneration, University M edical Center Göttingen, Göttingen, Germany

${ }^{3}$ Collaborative Research Center 889, U niversity of Göttingen, Göttingen, Germany

${ }^{4} \mathrm{Göttingen} \mathrm{Graduate} \mathrm{School} \mathrm{for} \mathrm{N} \mathrm{euroscience} \mathrm{and} \mathrm{M} \mathrm{olecular} \mathrm{Biosciences,} \mathrm{University} \mathrm{of} \mathrm{Göttingen,}$ Göttingen, Germany

IInstitute for Auditory Neuroscience and InnerEarLab, University Medical Center Göttingen, Göttingen, Germany

${ }^{6}$ Synaptic Nanophysiology Group, Max Planck Institute for Biophysical Chemistry, Göttingen, Germany

${ }^{7}$ Auditory Neuroscience Group, Max Planck Institute for Experimental Medicine, Göttingen, Germany

8Presynaptogenesis and Intracellular Transport in Hair Cells Group, Institute for Auditory N euroscience and InnerEarLab, University M edical Center Göttingen, Göttingen, Germany ${ }^{9}$ A uditory Systems Physiology Group and InnerEarLab, Department of O tolaryngology, U niversity of Göttingen M edical Center, Göttingen, Germany

*these authors contributed equally to the work "corresponding authors

PUBLICATION PROGRESS:

- Submitted to EM BO Journal $22^{\text {nd }}$ June 2018

- Resubmitted after revision $13^{\text {th }}$ November 2018

- Accepted $20^{\text {th }}$ D ecember 2018

- Published online $7^{\text {th }}$ February 2019 
MY CONTRIBUTIONS TO THE MANUSCRIPT:

\section{MY CONTRIBUTIONS}

\section{FIG. 1, 2, 4, 6}

FIG. $3 \quad$ P Panel D: Contribution to immunohistochemical staining of CtBP2

- Panels $C$ and E: immunohistochemical staining of CtBP2 and Cav1.3, STED microscopy and analysis using Igor Pro6 software (initially with the help of J.N.), statistics using Graphpad prism

○ Figure design together with L.M J.J.T.

FIG. $5 \quad$ Panel A: immunohistochemical staining of otoferlin, confocal microscopy

- Panel B: Otoferlin fluorescence intensity analysis using Imaris and $M$ atlab (the respective analysis routine was previously established (Strenzke et al., 2016), statistics

- Panel C: Intensity profile line scan analysis using I magel software

- Figure design

FIG. $7 \quad \circ$ Conventional electron microscopy embeddings, sectioning, staining, imaging

- A nalysis using I magej software and statistics using Graphpad prism

○ Figure design

FIG. $8 \quad \circ$ Electron microscopy and analysis (like Fig. 7)

- Electron tomography imaging, reconstruction of tomograms and analysis using IM OD software (initially with the help of C.W.), statistics

- Figure design

FIG. 9 Sample preparation (with $\mathrm{K}^{+}$stimulation), imaging and analysis like Fig. 7

○ Figure design

FIG. 10 Figure design (with help of C.V.)

FIG. EV $1,2,4$ -

FIG. EV3 3 Panel B: Myosin6 fluorescence intensity analysis using Imaris and M atlab, statistics (immunohistochemical staining and imaging performed by C.V.)

- Figure design

MANUSCRIPT Intensive editing (original draft written by T.M . and I.M .)

Please also see "A uthor contributions" in the manuscript. 
2.2 MANUSCRIPT 


\title{
Endophilin-A regulates presynaptic $\mathrm{Ca}^{2+}$ influx and synaptic vesicle recycling in auditory hair cells
}

\author{
Jana Kroll ${ }^{1,2,3,4, \dagger}$ (D), Lina M Jaime Tobón ${ }^{3,4,5,6,7, \dagger}$ D , Christian Vog $\left.\right|^{3,5,7,8, \dagger}$ (D), Jakob Neef ${ }^{3,5,6,7}$, \\ Ilona Kondratiuk ${ }^{1}$ D) , Melanie König ${ }^{1,3}$, Nicola Strenzke ${ }^{3,7,9}$ (D), Carolin Wichmann 2,3,7, ${ }^{*}$ (D), \\ Ira Milosevic ${ }^{1,3,{ }^{* *}}$ (D) \& Tobias Moser $3,5,6,7,{ }^{* * *}$ (iD
}

\begin{abstract}
Ribbon synapses of cochlear inner hair cells (IHCs) operate with high rates of neurotransmission; yet, the molecular regulation of synaptic vesicle (SV) recycling at these synapses remains poorly understood. Here, we studied the role of endophilins-A1-3, endocytic adaptors with curvature-sensing and curvature-generating properties, in mouse IHCs. Single-cell RT-PCR indicated the expression of endophilins-A1-3 in IHCs, and immunoblotting confirmed the presence of endophilin-A1 and endophilin-A2 in the cochlea. Patch-clamp recordings from endophilin-A-deficient IHCs revealed a reduction of $\mathrm{Ca}^{2+}$ influx and exocytosis, which we attribute to a decreased abundance of presynaptic $\mathrm{Ca}^{2+}$ channels and impaired SV replenishment. Slow endocytic membrane retrieval, thought to reflect clathrin-mediated endocytosis, was impaired. Otoferlin, essential for IHC exocytosis, co-immunoprecipitated with purified endophilin-A1 protein, suggestive of a molecular interaction that might aid exocytosis-endocytosis coupling. Electron microscopy revealed lower SV numbers, but an increased occurrence of coated structures and endosome-like vacuoles at IHC active zones. In summary, endophilins regulate $\mathrm{Ca}^{2+}$ influx and promote SV recycling in IHCs, likely via coupling exocytosis to endocytosis, and contributing to membrane retrieval and SV reformation.
\end{abstract}

Keywords electron microscopy; endocytosis; membrane capacitance; ribbon synapse; super-resolution microscopy

Subject Categories Neuroscience

DOI 10.15252/embj.2018100116 | Received 22 June 2018 | Revised 17

December 2018| Accepted 20 December 2018

The EMBO Journal (2019) e100116

\section{Introduction}

Ribbon synapses of auditory IHCs faithfully convert acoustic signals into an action potential code in spiral ganglion neurons (SGNs). Individual presynaptic active zones (AZs) of IHCs are thought to drive firing in a single SGN at rates of up to hundreds of $\mathrm{Hz}$ for as long as the sound continues (Safieddine et al, 2012; Wichmann \& Moser, 2015; Rutherford \& Moser, 2016). Exocytosis of synaptic vesicles (SVs) at IHC AZs is both, fast and indefatigable. It seems to operate independently of classical neuronal SNARE proteins, Munc13-like priming factors, or complexins (Strenzke et al, 2009; Nouvian et al, 2011; Vogl et al, 2015), but, instead, involves the deafness gene product otoferlin (Roux et al, 2006; Pangrsic et al, 2010). Probably the most important coordinator of synaptic transmission is $\mathrm{Ca}^{2+}$ that enters IHCs primarily through presynaptic voltage-gated $\mathrm{Ca}_{V} 1.3 \mathrm{Ca}^{2+}$ channels and mediates excitation-secretion coupling (Platzer et al, 2000; Brandt et al, 2003, 2005; Weiler et al, 2014; Wong et al, 2014). At IHC AZs, $\mathrm{Ca}^{2+}$ channels are present in defined numbers, organized in a stripe-like manner, and show little inactivation, which enables reliable information transfer during sustained stimulation (Brandt et al, 2005; Frank et al, 2009, 2010; Ohn et al, 2016; Neef et al, 2018). Interestingly, $\mathrm{Ca}^{2+}$ channels have been reported to interact with otoferlin (Ramakrishnan et al, 2009) and with endophilins (Chen et al, 2003), the protein family under study here.

To sustain high transmission rates, IHCs need to balance exocytosis by equally efficient SV recycling (Siegel \& Brownell, 1986; Parsons et al, 1994; Moser \& Beutner, 2000; Beutner et al, 2001; Lenzi et al, 2002; Trapani et al, 2009; Neef et al, 2014; Revelo et al, 2014; Jung et al, 2015). Here, at least three kinetically distinct forms

1 Synaptic Vesicle Dynamics Group, European Neuroscience Institute (ENI), University Medical Center Göttingen, Göttingen, Germany

2 Molecular Architecture of Synapses Group, Institute for Auditory Neuroscience, InnerEarLab and Center for Biostructural Imaging of Neurodegeneration, University Medical Center Göttingen, Göttingen, Germany

3 Collaborative Research Center 889, University of Göttingen, Göttingen, Germany

4 Göttingen Graduate School for Neuroscience and Molecular Biosciences, University of Göttingen, Göttingen, Germany

5 Institute for Auditory Neuroscience and InnerEarLab, University Medical Center Göttingen, Göttingen, Germany

6 Synaptic Nanophysiology Group, Max Planck Institute for Biophysical Chemistry, Göttingen, Germany

7 Auditory Neuroscience Group, Max Planck Institute for Experimental Medicine, Göttingen, Germany

8 Presynaptogenesis and Intracellular Transport in Hair Cells Group, Institute for Auditory Neuroscience and InnerEarLab, University Medical Center Göttingen, Göttingen, Germany

9 Auditory Systems Physiology Group and InnerEarLab, Department of Otolaryngology, University of Göttingen Medical Center, Göttingen, Germany *Corresponding author. Tel: +49 55139 61128; Fax: +49 551 3912950; E-mail: carolin.wichmann@med.uni-goettingen.de

**Corresponding author. Tel: +49 551 3912379; Fax: +49 551 3912346; E-mail: i.milosevic@eni-g.de

***Corresponding author. Tel: +49 55139 63070; Fax: +49 551 3912950; E-mail: tmoser@gwdg.de

${ }^{\dagger}$ These authors contributed equally to this work 
of endocytic membrane retrieval-rapid (300 ms), fast (4 s), and slow (20 s half-time recovery) - have been described for IHCs (Moser \& Beutner, 2000; Beutner et al, 2001; Neef et al, 2014). However, to date, knowledge of the molecular entities mediating these kinetically distinct forms of endocytosis in IHCs remains scarce. In line with findings at conventional synapses (Ferguson \& De Camilli, 2012; Kononenko \& Haucke, 2015), work on endocytosis in IHCs has indicated a role of dynamins (Boumil et al, 2010; Neef et al, 2014), synaptojanin-1 (Trapani et al, 2009), and clathrin (Siegel \& Brownell, 1986; Neef et al, 2014; Jung et al, 2015) in slow endocytosis that most likely represents clathrin-mediated endocytosis [CME, recently reviewed in Pangrsic and Vogl (2018)].

Surprisingly, genetic disruption of the clathrin adaptor AP-2 did not noticeably affect endocytic membrane retrieval in IHCs (Jung et al, 2015). However, in AP- $2 \mu$ mutants, the abundance of clathrincoated structures near the presynaptic AZs was reduced and large membranous organelles (endosome-like vacuoles, ELVs) accumulated after stimulation (Jung et al, 2015) similar to findings at conventional synapses (Kononenko et al, 2014). This supports the notion that, next to CME, bulk retrieval (reviewed in Kokotos and Cousin (2015)) may play a prominent role in hair cells (Lenzi et al, 2002; Neef et al, 2014; Revelo et al, 2014; Jung et al, 2015). SVs are then rapidly reformed from endocytosed membranes (Kamin et al, 2014; Revelo et al, 2014), which seems to employ clathrin-dependent and clathrin-independent mechanisms (Jung et al, 2015). Importantly, the processes of exocytosis and endocytosis are intimately coupled and tightly coordinated-both at classical neuronal and IHC ribbon synapses - and the proper function of both types of synapses depends on this coupling (Haucke et al, 2011; Wichmann \& Moser, 2015; Milosevic, 2018). In IHCs, AP-2, which interacts with otoferlin (Duncker et al, 2013; Jung et al, 2015), has been implicated in exocytosis and endocytosis coupling (Jung et al, 2015).

In neurons, a range of molecular key players have been identified that orchestrate endocytic membrane retrieval and SV reformation (Kononenko \& Haucke, 2015; Milosevic, 2018); yet, their respective relevance for these processes in IHCs remains unclear. In this context, one interesting molecular target is the evolutionary conserved family of endophilin-A proteins (henceforth "endophilin”), which are involved in endocytic membrane retrieval and uncoating in neurons of invertebrates (Verstreken et al, 2002, 2003; Schuske et al, 2003) and mammals (Milosevic et al, 2011; Watanabe et al, 2018). The current view on mammalian endophilins (A1-A3) pictures them as hubs of a protein network that coordinates cargo packing, bud constriction, actin assembly, and recruitment of factors needed for fission and uncoating (Saheki \& Camilli, 2012). Structurally, endophilins contain a BAR domain that senses and induces membrane curvature, as well as a SH3 domain that recruits the GTPase dynamin and the $\mathrm{PI}(4,5) \mathrm{P}_{2}$ phosphatase synaptojanin-1 to clathrin-coated pits (Verstreken et al, 2002, 2003; Schuske et al, 2003; Perera et al, 2006; Ferguson et al, 2009; Simunovic et al, 2017). Upon fission, $\mathrm{PI}(4,5) \mathrm{P}_{2}$ degradation initiates the shedding of clathrin adaptor proteins from the endocytosed membranes, ultimately leading to the uncoating of SVs (Schuske et al, 2003; Verstreken et al, 2003; Milosevic et al, 2011; Pechstein et al, 2015; Watanabe et al, 2018).

To clarify a potential contribution of endophilins in IHC presynaptic physiology, we performed a comprehensive functional and morphological analysis encompassing single-cell RT-PCR, immunoblotting, electron microscopy, immunohistochemistry, patch-clamp recordings, biochemical interaction studies, and auditory systems physiology using constitutive endophilin knockout mice.

\section{Results}

\section{All three endophilins are expressed in the cochlea}

To investigate the expression of endophilin genes in the organ of Corti and, more specifically, in IHCs, we collected mRNA from IHCs of the apical cochlear coil of Wt mice (C57BL/6J, 2 weeks old, i.e., right after hearing onset). After reverse transcription, we performed single-cell multiplex-nested real-time PCR. In these experiments, all three endophilin-A transcripts (i.e., A1, A2, and A3; Fig 1A and B) could be detected in all tested single IHC samples that were also positive for the housekeeping gene HPRT (see Appendix Table S1 for primer sequences). Importantly, we could not detect endophilin mRNAs in our negative control samples, i.e., a small volume of bath solution that was collected in close proximity to the IHC row prior to and directly after the extraction of the IHC cytoplasm. We note that, while this approach does not provide a quantitative assessment of expression levels due to the nature of the amplification procedure with nested primer pairs, it reliably indicates the presence of endophilin-A1-3 mRNAs in IHCs.

To further investigate endophilin protein expression in situ, we have tested several commercially available as well as custom-made anti-endophilin-A antibodies (see Materials and Methods) in various fixation and permeabilization conditions; yet, we did not obtain specific immunolabeling in the organ of Corti. Therefore, we proceeded to perform immunoblotting with KO-verified antibodies (Milosevic et al, 2011) on cochlear samples of all genotypes using hippocampal and cerebellar tissue extracts as positive controls. Here, we detected bands with the expected molecular weight of endophilin-A1 ( $\sim 39 \mathrm{kD})$ and endophilin-A2 $(\sim 42 \mathrm{kD})$ in $\mathrm{Wt}$ cochleae. These bands were absent in cochlear lysates of the respective KO genotypes, hence strongly suggesting target specificity (Fig 1C- $\mathrm{C}^{\prime} ; \gamma$-adaptin was used as an independent loading control). Taken together, these data indicate the expression of endophilinsA1-3 in IHCs and show the presence of endophilin-A1 and endophilin-A2 protein in the murine cochlea.

\section{Endophilin promotes $\mathrm{Ca}^{2+}$ influx and efficient SV replenishment in IHCs}

Next, we employed perforated patch-clamp recordings to assess the role of endophilins in presynaptic IHC function. Since the cumulative loss of all three endophilin genes is perinatally lethal, we first prepared organotypic cultures of organs of Corti harvested from endophilin-A1/2/3 triple KO (TKO; see Materials and Methods for exact genetic descriptions and breeding schemes of endophilin mutants) and endophilin-A1/3 double KO (1/3DKO) mice, as well as C57BL/6J pups within 3-8 h after birth. Thereafter, organs of Corti were maintained in culture for 1 week to enable synaptic maturation and the otoferlin-dependence of exocytosis to be established (Sobkowicz et al, 1982; Vogl et al, 

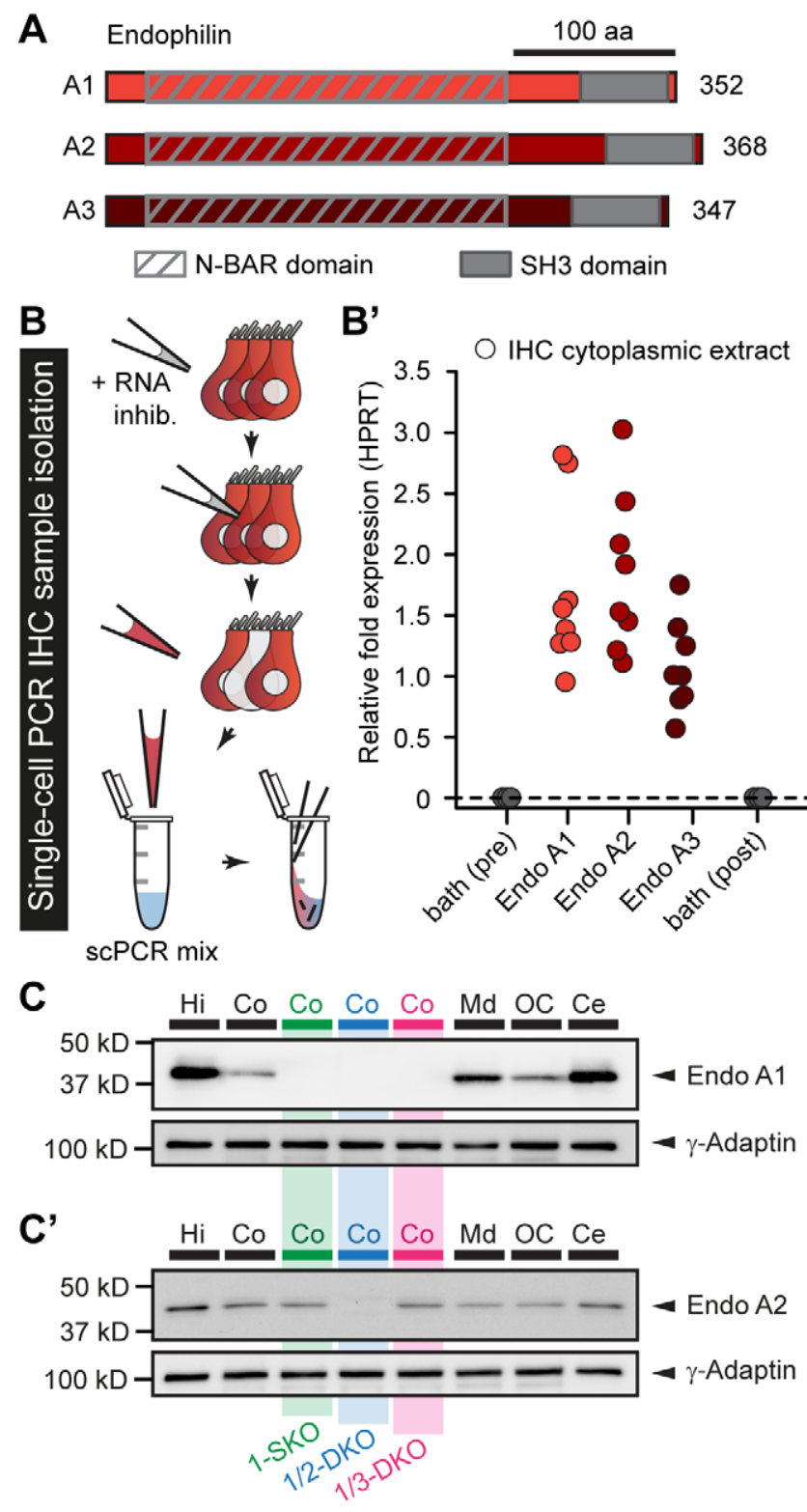

2015) prior to detailed electrophysiological analysis (Fig $2 A^{\prime}$ ). In order to boost depolarization-induced exocytosis, whole-cell $\mathrm{Ca}^{2+}$ currents $\left(\mathrm{I}_{\mathrm{Ca}}\right)$ and the ensuing exocytic membrane capacitance changes $\left(\Delta \mathrm{C}_{\mathrm{m}}\right)$ were recorded at an elevated extracellular $\mathrm{Ca}^{2+}$ concentration of $10 \mathrm{mM}\left(\left[\mathrm{Ca}^{2+}\right]_{\mathrm{e}} ; 1.3 \mathrm{mM}\right.$ is considered physiological). In these experiments, IHCs of $1 / 3$-DKOs exhibited a $25 \%$ reduction of the presynaptic $\mathrm{I}_{\mathrm{Ca}}$ and TKO IHCs showed a nonsignificant trend toward smaller $\mathrm{I}_{\mathrm{Ca}}$ (Fig 2A and $\mathrm{A}^{\prime \prime}, \mathrm{I}_{\max }$ : $-317 \pm 27.3 \mathrm{pA}$ for TKO IHCs, $-298 \pm 22.9 \mathrm{pA}$ for $1 / 3-\mathrm{DKO}$, and $-403 \pm 32.2 \mathrm{pA}$ for $\mathrm{Wt}$; one-way ANOVA, $F(2,26)=3.89$, $P=0.0334$; post hoc Tukey's test: $P=0.046 \mathrm{Wt}$ versus $1 / 3-\mathrm{DKO}$; $P=0.103 \mathrm{Wt}$ versus TKO for the maximal $\mathrm{I}_{\mathrm{Ca}}$ elicited by depolarization to $-17 \mathrm{mV}$ ). Interestingly, $\mathrm{I}_{\mathrm{Ca}}$ of $\mathrm{TKO}$ IHCs showed enhanced inactivation, as evident from a significantly reduced fraction of $\mathrm{I}_{\mathrm{Ca}}$ remaining at $100 \mathrm{~ms}$ of depolarization, which was

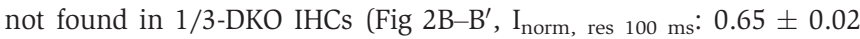
for TKO IHCs, $0.73 \pm 0.02$ for $1 / 3-\mathrm{DKO}$, and $0.71 \pm 0.02$ for Wt;
Figure 1. Endophilin-A expression in the cochlea.

A Schematic domain overview of endophilins-A1-3, highlighting the BAR and $\mathrm{SH} 3$ domains.

B-B' Schematic overview of the sample collection procedure for single-cell RT-PCR (SCPCR). Single IHC cytoplasms from acutely dissected organs of Corti of C57BL/6J (Wt) mice after hearing onset were aspirated and processed for SCPCR as depicted. ( $\left.B^{\prime}\right)$ Expression analysis of endophilinsA1-3 from individually isolated IHC cytoplasms using RT-PCR from a representative experimental run. Please note that for these experiments, negative bath control samples from before and after the isolation procedure were an essential requirement to ensure lack of contamination from cellular debris in the bath solution. HPRT was used as a housekeeping gene.

C-C' Immunoblotting of tissue lysates from postnatal day (p) $15 \mathrm{Wt}(\mathrm{C} 57 \mathrm{BL} / 6 \mathrm{6})$, 1-SKO, 1/2-DKO, and 1/3-DKO revealed protein expression of endophilin$\mathrm{A} 1$ and endophilin-A2 in the murine cochlea and ensured antibody specificity. Unfortunately, none of the commercially available endophilin-A3 antibodies we tested in these experiments gave a specific signal for A3 in cochlear extracts, but rather appeared to (also) detect A1. $\gamma$-Adaptin was used as loading control. All antibody epitopes localize to the distinct $\mathrm{C}$-terminal regions of the different endophilin-A family members. $\mathrm{Hi}$, hippocampus; Ce, cerebellum; Co, pooled cochleae from a single individual of the indicated genotype; Md, modioli (micro-dissected and pooled from $10 \mathrm{Wt}$ animals); OC, organs of Corti (micro-dissected and pooled from $10 \mathrm{Wt}$ animals).

Source data are available online for this figure.

one-way ANOVA, $F(2,26)=4.89, P=0.0158$; post hoc Tukey's test: $P=0.046$ for TKO versus Wt; $P=0.794$ for $1 / 3$-DKO versus $\mathrm{Wt}$ ). Such $\mathrm{I}_{\mathrm{Ca}}$ reduction and enhanced $\mathrm{I}_{\mathrm{Ca}}$ inactivation suggest $\mathrm{a}$ functional interaction of endophilins and $\mathrm{Ca}^{2+}$ channel complexes, which is in line with previous biochemical interaction studies (Chen et al, 2003).

Recordings of exocytic changes in membrane capacitance $\left(\Delta \mathrm{C}_{\mathrm{m}}\right)$ showed impaired exocytosis. Exocytosis of the readily releasable pool (RRP), as approximated by $\Delta \mathrm{C}_{\mathrm{m}}$ responses to 20 ms depolarizations, was significantly attenuated in $1 / 3$ DKO IHCs and tended to be reduced in TKO IHCs (one-way ANOVA, F(3, $33)=5.35, P=0.0041$; post hoc Tukey's test: $P=0.075$ for $\mathrm{Wt}$ versus TKO; $P=0.006$ for $\mathrm{Wt}$ versus $1 / 3-\mathrm{DKO}$ ). Similarly, sustained exocytosis, probed by 100-ms-long depolarizations, tended to be attenuated in both genotypes (Fig 2C-C", KruskalWallis statistic $(\mathrm{KWS})=10.93, P=0.0121$; post hoc Dunn's test: $P=0.220$ for Wt versus TKO; $P=0.025$ for $\mathrm{Wt}$ versus $1 / 3$-DKO). In contrast, no significant difference was found for responses to short stimuli ( $<10 \mathrm{~ms}$, also see Appendix Tables S2 and S3), indicating that endophilins are dispensable for SV fusion. In order to disentangle the reduction of exocytosis caused by diminished $\mathrm{I}_{\mathrm{Ca}}$ from a potential impairment of SV replenishment in the absence of endophilin-A1 and endophilin-A3, we attempted to match the decreased $\mathrm{I}_{\mathrm{Ca}}$ amplitudes by performing additional recordings from $\mathrm{Wt}(\mathrm{C} 57 \mathrm{BL} / 6 \mathrm{~J}) \mathrm{IHCs}$ at lower $\left[\mathrm{Ca}^{2+}\right]_{\mathrm{e}}$ (i.e., $6 \mathrm{mM}$ instead of $10 \mathrm{mM}$; Fig $2 \mathrm{C}^{\prime}-\mathrm{D}$ ). Under these conditions, $\mathrm{I}_{\mathrm{Ca}}$ of $\mathrm{Wt}$ IHCs closely resembled the ones of endophilin-deficient mutant IHCs. However, the extent of exocytosis from Wt IHCs still exceeded that of cultured 1/3-DKO and TKO IHCs for depolarizations $\geq 10 \mathrm{~ms}$ and remained comparable to the data acquired at $10 \mathrm{mM}\left[\mathrm{Ca}^{2+}\right]_{\mathrm{e}}$ (Fig $\left.2 \mathrm{C}^{\prime}-\mathrm{C}^{\prime \prime}\right)$. Hence, the reduction in $\mathrm{I}_{\mathrm{Ca}}$ in the endophilin mutants cannot fully account for the observed impairment of exocytosis, suggesting an additional requirement for 
A

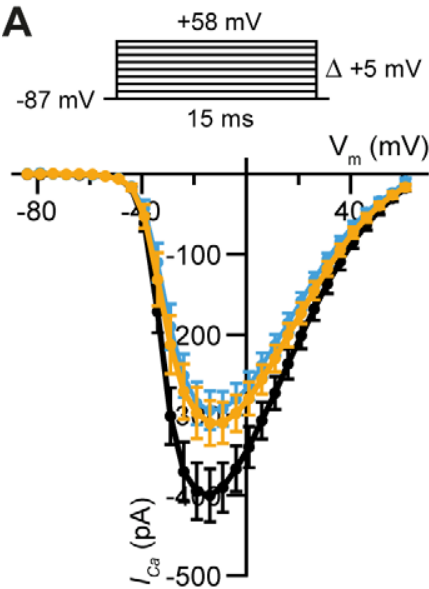

B
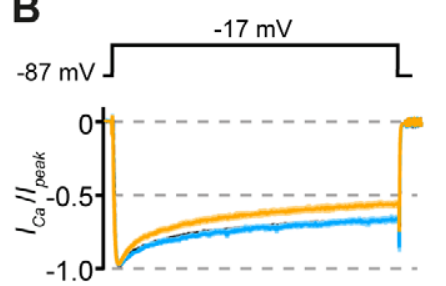

$20 \mathrm{~ms}$
A'
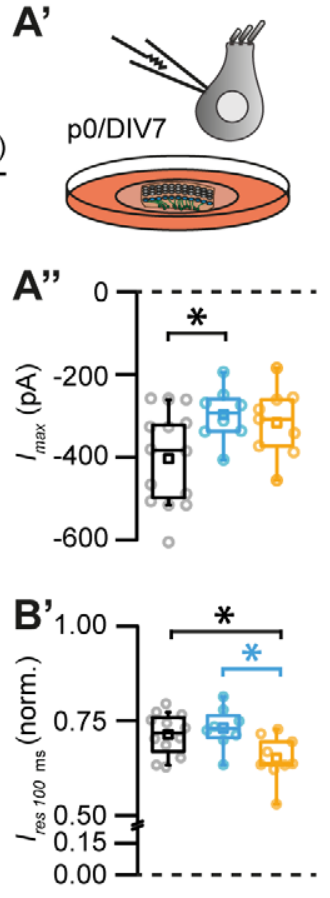
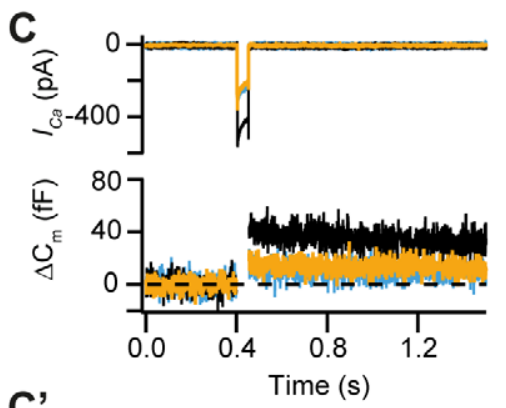

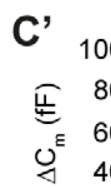
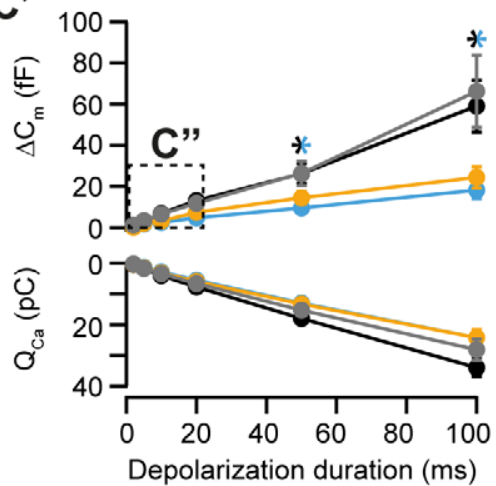

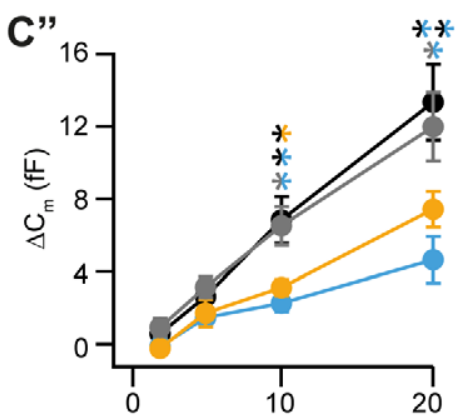

Depolarization duration ( $\mathrm{ms}$ )

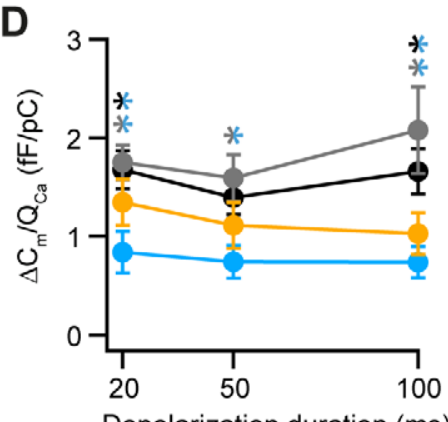

Wt $10 \mathrm{mM}\left[\mathrm{Ca}^{2+}\right]_{e}(\mathrm{n}=12 ; \mathrm{N}=9 ; \mathrm{C}=5)$

Wt $6 \mathrm{mM}\left[\mathrm{Ca}^{2+}\right]_{e}(n=8 ; N=5 ; C=4)$

$\rightarrow$ 1/3-DKO $10 \mathrm{mM}\left[\mathrm{Ca}^{2+}\right]_{e}(\mathrm{n}=8 ; \mathrm{N}=7 ; \mathrm{C}=3)$

1/2/3-TKO $10 \mathrm{mM}\left[\mathrm{Ca}^{2+}\right]_{\mathrm{e}}(\mathrm{n}=9 ; \mathrm{N}=7 ; \mathrm{C}=4)$

Figure 2. Reduced presynaptic $\mathrm{Ca}^{2+}$ currents and exocytosis in endophilin-deficient IHCs maintained in organotypic culture.

A-A" Ca ${ }^{2+}$ current-voltage relationships evoked by incremental $15-\mathrm{ms}$ step depolarizations from $-87 \mathrm{mV}$ to $+58 \mathrm{mV}$ revealed a $\sim 25 \%$ reduction of $\mathrm{I}_{\mathrm{Ca}}$ in $1 / 3 \mathrm{DKO}$ and TKO mice. $\left(A^{\prime}\right)$ Due to the perinatal lethality of TKO mice, detailed electrophysiological characterization of TKO IHCs had to be performed on organotypically cultured organs of Corti after 7 days in vitro (DIV). C57BL/6] (Wt) and 1/3-DKO served as controls. Please note that all recordings from cultured IHCS were performed at $\left[\mathrm{Ca}^{2+}\right]_{\mathrm{e}}$ of $10 \mathrm{mM}$ to maximize IHC exocytic performance. (A") Quantification and statistical analysis of individual maximum $\mathrm{I}_{\mathrm{Ca}}$ amplitudes $\left(I_{\max }\right)$ of the respective genotypes revealed a significant reduction in $I_{\max }$ in both endophilin mutant genotypes $\left({ }^{*} P=0.046\right.$, one-way ANOVA with post hoc Tukey's test).

B-B $\mathrm{B}^{\prime} \quad \mathrm{Ca}^{2+}$ current inactivation was probed by test pulses of $100 \mathrm{~ms}$ to the $I_{\max }$ potential and revealed a significantly stronger inactivation phenotype in TKO IHCs when directly compared to Wt and 1/3-DKO cells. (B') Quantification and statistical analysis of the residual current $\left(I_{\text {res }} 100 \mathrm{~ms}\right)$ at the end of the test pulse ${ }^{\star} P=0.046$, one-way ANOVA with post hoc Tukey's test).

$C-C^{\prime \prime}$ Representative $\mathrm{I}_{\mathrm{Ca}}$ (upper panel) and $\mathrm{C}_{\mathrm{m}}$ (lower panel) in response to a $50 \mathrm{~ms}$ depolarizing step to the potential eliciting $I_{\max }$. $\left(\mathrm{C}^{\prime}\right)$ Exocytic $\Delta \mathrm{C}_{\mathrm{m}}$ and corresponding $\mathrm{Q}_{\mathrm{ca}}$ elicited by depolarizations of stimulus durations from 2 to $100 \mathrm{~ms}$ for all respective genotypes and at $\left[\mathrm{Ca}^{2+}\right]_{\mathrm{e}}=6 \mathrm{mM}$ for a second set of recordings from wild-type IHCS to experimentally approximate the decreased $I_{C a}$ observed in the endophilin mutants. $\left(C^{\prime \prime}\right)$ Magnification of the initial, short depolarizing steps (2$20 \mathrm{~ms}$ ) for clarity. Exocytic $\Delta C_{m}$ of cultured endophilin-deficient IHCs was strongly reduced ( ${ }^{*} P<0.05$; ${ }^{\star *} P<0.01$; one-way ANOVA with post hoc Tukey's or nonparametric K-W with post hoc Dunn's test; please also refer to Appendix Tables S2 and S3 for detailed statistical analysis).

D The reduced $\mathrm{Ca}^{2+}$ efficiency of exocytosis $\left(\Delta \mathrm{C}_{\mathrm{m}} / \mathrm{Q}_{\mathrm{Ca}}\right)$ in endophilin-deficient IHCs indicates that diminished $\mathrm{Ca}^{2+}$ influx cannot fully account for the reduction of exocytosis $\left({ }^{*} P<0.05\right.$; ${ }^{* *} P<0.01$; one-way ANOVA with post hoc Tukey's or non-parametric K-W with post hoc Dunn's test; please also refer to Appendix Table $S 4$ for detailed statistical analysis).

Data information: For panels (A-D), the following numbers of replicates were used: Wt $10 \mathrm{mM}\left[\mathrm{Ca}^{2+}\right]_{\mathrm{e}}$ number of cells $(n)=12$, number of animals $(\mathrm{N})=9$, number of organotypic cultures (C) $=5$; Wt $6 \mathrm{mM}\left[\mathrm{Ca}^{2+}\right]_{\mathrm{e}} n=8 / \mathrm{N}=5 / \mathrm{C}=4$; 1/3-DKO $10 \mathrm{mM}\left[\mathrm{Ca}^{2+}\right]_{\mathrm{e}} n=8 / \mathrm{N}=7 / \mathrm{C}=3$; TKO $10 \mathrm{mM}\left[\mathrm{Ca}{ }^{2+}\right]_{\mathrm{e}} n=9 / \mathrm{N}=7 / \mathrm{C}=4$. Error bars in $\left(\mathrm{C}^{\prime}-\mathrm{D}\right)$ indicate the SEM; box plots in $\left(\mathrm{A}^{\prime \prime}\right)$ and $\left(\mathrm{B}^{\prime}\right)$ illustrate the median with the interquartile range, whiskers indicate $10-90 \%$ of data points, and the squares present the respective mean value.

endophilin-A1 and endophilin-A3 in exocytosis, e.g., in vesicle replenishment. This is further illustrated by plotting $\Delta \mathrm{C}_{\mathrm{m}}$ versus the corresponding charge of $\mathrm{I}_{\mathrm{Ca}}$, which shows a reduced efficiency of $\mathrm{I}_{\mathrm{Ca}}$ to drive exocytosis (Fig 2D; individual statistics for all depolarization durations can be found in Appendix Table S4). Taken together, patch-clamp analysis of organotypically cultured IHCs from endophilin mutants revealed reduced $\mathrm{I}_{\mathrm{Ca}}$ amplitudes alongside altered $\mathrm{I}_{\mathrm{Ca}}$ inactivation and impaired exocytosis. Moreover, our data imply a partial functional dispensability of endophilin-A2 in the presence of endophilin-A1 and endophilin-A3, since both $\mathrm{I}_{\mathrm{Ca}}$ and $\Delta \mathrm{C}_{\mathrm{m}}$ amplitudes were statistically indistinguishable between 1/3-DKO and TKO IHCs.

Guided by these results, we focused our further perforated patchclamp studies on $\mathrm{I}_{\mathrm{Ca}}$ and exocytosis of 1-SKO and 1/3-DKO IHCs after hearing onset (at p14-17) and at near physiological $\left[\mathrm{Ca}^{2+}\right]_{\mathrm{e}}$ ( $2 \mathrm{mM})$. Analogous to our observations from cultured immature IHCs, we found a statistically significant reduction of $\mathrm{I}_{\mathrm{Ca}}$ of $\sim 20 \%$ for 1-SKO and 1/3-DKO IHCs under these conditions (Fig 3A-A'; $\mathrm{I}_{\max }:-128 \pm 5.0 \mathrm{pA}$ for 1 -SKO IHCs, $-129 \pm 2.1 \mathrm{pA}$ for $1 / 3-\mathrm{DKO}$, and $-153 \pm 5.0 \mathrm{pA}$ for Wt; KWS $=18.72 ; P<0.0001$; post hoc 

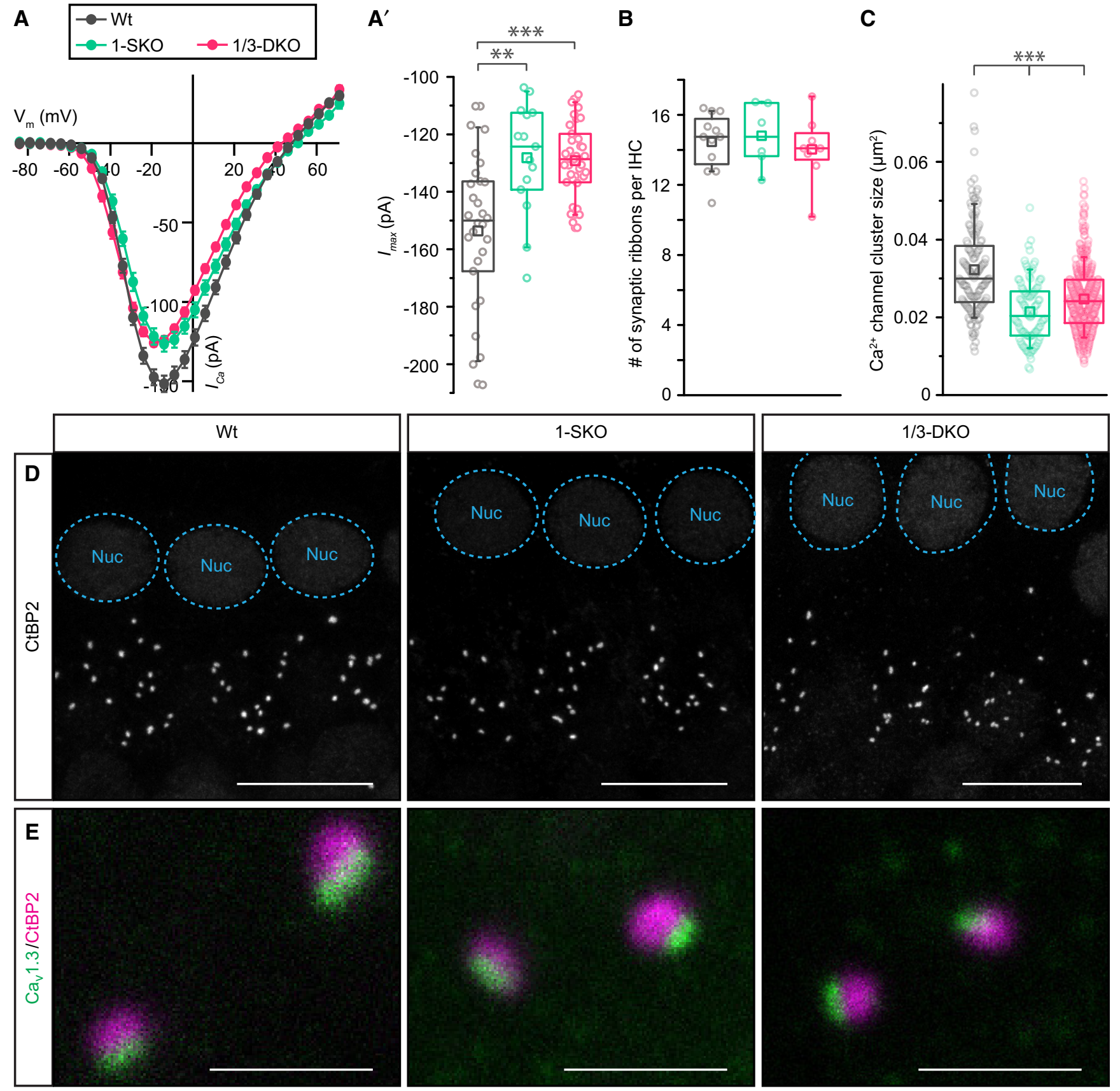

Figure 3. Disruption of endophilins reduces $\mathrm{Ca}^{2+}$ influx of $\mathrm{IHCs}$ after hearing onset.

A Ca ${ }^{2+}$ current-voltage relationships in response to 10-ms step depolarizations. ( $\left.A^{\prime}\right)$ The peak of $\mathrm{Ca}^{2+}$ influx was significantly reduced in endophilin 1 -SKO

$\left.{ }^{* *} P=0.0024\right)$ and $1 / 3-$ DKO $\left.{ }^{* \star \star} P=0.0003\right)$ when compared to Wt $(\mathrm{Wt} n=30 / \mathrm{N}=20 ; 1-\mathrm{SKO} n=15 / \mathrm{N}=9 ; 1 / 3-\mathrm{DKO} n=39 / \mathrm{N}=20$; non-parametric K-W with post hoc Dunn's correction).

B Quantification of CtBP2-labeled ribbons revealed a number of approximately 14-15 ribbon synapses per IHC comparable across all genotypes (Wt $n=12 / \mathrm{N}=9$; 1SKO $n=6 / N=4 ; 1 / 3-$ DKO $n=8 / N=6$; one-way ANOVA, $P=0.717$ ).

C Quantification of the cross-sectional area of Cav1.3 immunofluorescence revealed approx. 34\% smaller clusters in 1-SKOs and 24\% smaller clusters in 1/3-DKOs (Wt $n=153 / \mathrm{N}=2 ; 1-\mathrm{SKO} n=102 / \mathrm{N}=2 ; 1 / 3-\mathrm{DKO} n=441 / \mathrm{N}=4 ;{ }^{* \star *} \mathrm{P}<0.0001 ; \mathrm{K}-\mathrm{W}$ and post hoc Dunn's test).

D Representative maximum projections of confocal sections from organs of Corti of p15 Wt, 1-SKO, and 1/3-DKO mice stained for CtBP2 to label synaptic ribbons. Please note that CtBP2 expression is also found in the nucleus (nuclei are highlighted by dashed circles in the individual panels). Scale bars: $10 \mu \mathrm{m}$.

E 2D STED images of IHC ribbon synapses stained for CtBP2 (magenta) and Cav1.3 (green). Cav1.3 Ca ${ }^{2+}$ channels remain clustered at AZs of 1-SKO and 1/3-DKO IHCs. Scale bars: $1 \mu \mathrm{m}$.

Data information: Box plots in $\left(A^{\prime}-C\right)$ illustrate the median with the interquartile range, whiskers indicate $10-90 \%$ of data points, and the squares present the respective mean value. 
Dunn's test: $P=0.0024$ for Wt versus 1 -SKO and $P=0.0003$ for $\mathrm{Wt}$ versus $1 / 3$-DKO for the maximal $\mathrm{I}_{\mathrm{Ca}}$ elicited by depolarization to $-14 \mathrm{mV}) . \mathrm{I}_{\mathrm{Ca}}$ inactivation at $200 \mathrm{~ms}$ of depolarization was not altered in 1-SKO, 1/2 DKO, and 1/3-DKO IHCs (Fig EV1; $\mathrm{I}_{\text {norm, res } 200 \mathrm{~ms}}: 0.72 \pm 0.02$ for 1 -SKO IHCs, $0.74 \pm 0.01$ for $1 / 2$-DKO, $0.73 \pm 0.02$ for $1 / 3$-DKO, and $0.70 \pm 0.02$ for Wt; one-way ANOVA, $F(3,55)=0.95, P=0.4218)$, likely suggesting functional redundancy between the different endophilin genes.

In order to explore potential reasons for the observed reduction of $\mathrm{I}_{\mathrm{Ca}}$, such as a decreased number of IHC synapses or a lower abundance of $\mathrm{Ca}_{\mathrm{V}} 1.3 \mathrm{Ca}^{2+}$ channels per $\mathrm{AZ}$, we performed semi-quantitative immunohistochemistry on IHCs from 2-week-old Wt, 1-SKO, and 1/3-DKO mice. Firstly, we counted the number of synaptic ribbons per IHC as a proxy of the number of afferent synapses (Khimich et al, 2005; Meyer et al, 2009, Fig 3B and D) and found no difference between the genotypes (one-way ANOVA, $F(2,23)=0.337$, $P=0.717)$. Secondly, all ribbons appeared anchored at the $\mathrm{AZ}$ (Fig 3E and electron microscopy data in Fig 8A, see below), where presynaptic $\mathrm{Ca}^{2+}$ channels remained clustered (Fig 3E). In line with the reduced $\mathrm{I}_{\mathrm{Ca}}$ amplitudes, super-resolution 2D STED imaging of $\mathrm{Ca}_{\mathrm{V}} 1.3$ immunofluorescence revealed a significant decrease in crosssectional area of presynaptic $\mathrm{Ca}^{2+}$ channel clusters of 1-SKO and 1/3DKO IHCs (Fig 3C and E; Wt: $0.032 \pm 0.001 \mu \mathrm{m}^{2}$ versus 1-SKO: $0.021 \pm 0.001 \mu \mathrm{m}^{2}$ versus $1 / 3$-DKO: $0.024 \pm 0.001 \mu \mathrm{m}^{2} ; \mathrm{KWS}=$ 76.14, $P<0.0001$; post hoc Dunn's test: $P<0.0001$ for Wt versus 1 -SKO and $P<0.0001$ for Wt versus $1 / 3$-DKO).

Next, we tested the exocytic capacity of endophilin-deficient IHC ribbon synapses after the onset of hearing by measuring exocytic $\Delta \mathrm{C}_{\mathrm{m}}$ in response to step depolarizations to $-14 \mathrm{mV}$ eliciting maximal $\mathrm{I}_{\mathrm{Ca}}$ at near physiological $\left[\mathrm{Ca}^{2+}\right]_{\mathrm{e}}(2 \mathrm{mM}$, Fig 4$)$. In line with our observations from organotypically cultured IHCs, we found sustained exocytosis to be reduced in 1/3-DKO IHCs (Fig 4A and B; e.g., $\Delta \mathrm{C}_{\mathrm{m}, 200 \mathrm{~ms}}$ for $1 / 3$-DKO IHCs was $55.11 \pm 4.8 \mathrm{fF}$ versus $88.16 \pm 13.11 \mathrm{fF}$ for Wt IHCs; KWS $=6.03, P=0.0490$; post hoc Dunn's test: $P=0.0470$ for $1 / 3$-DKO versus Wt; individual Tukey's/ post hoc Dunn's results for all other depolarization durations can be found in Appendix Tables S5 and S6). Likewise, when approximating the rate of sustained exocytosis as $\left(\Delta \mathrm{C}_{\mathrm{m}}, 200 \mathrm{~ms}-\Delta \mathrm{C}_{\mathrm{m}, 20 \mathrm{~ms}}\right) /$ $180 \mathrm{~ms}$, we found a tendency for lower rates in endophilin-deficient IHCs $(0.25 \pm 0.02 \mathrm{fF} / \mathrm{ms}$ for $1 / 3-\mathrm{DKO}, 0.27 \pm 0.03 \mathrm{fF} / \mathrm{ms}$ for $1-\mathrm{SKO}$ versus $0.42 \pm 0.06 \mathrm{fF} / \mathrm{ms}$ for $\mathrm{Wt}$; KWS $=5.223, P=0.0734)$. This trend remained also after normalizing to the integrated $\mathrm{I}_{\mathrm{Ca}}\left(\mathrm{Q}_{\mathrm{Ca}}\right)$, suggesting an exocytic deficit beyond that explained by reduced $\mathrm{Ca}^{2+}$ influx, as already seen in cultured IHCs (Fig 4C; e.g., for $200 \mathrm{~ms}: 2.54 \pm 0.19 \mathrm{fF} / \mathrm{pC}$ for $1 / 3$-DKO versus $3.34 \pm 0.52 \mathrm{fF} / \mathrm{pC}$ for 1-SKO versus $3.81 \pm 0.45 \mathrm{fF} / \mathrm{pC}$ for $\mathrm{Wt}$; $\mathrm{KWS}=6.70, P=0.0352$, post hoc Dunn's test: $P=0.0323$ for $1 / 3-\mathrm{DKO}$ versus $\mathrm{Wt}$ and $P>0.99$ for 1-SKO versus Wt; individual post hoc Tukey's/Dunn's results for all other depolarization durations can be found in Appendix Table S7).

Finally, to assess the consequences of endophilin disruption at the level of the auditory system, we recorded auditory brainstem responses (ABRs) from 1-SKO, 1/3-DKO and Wt mice at 6 weeks of age. ABRs reflect the synchronous activation of auditory neurons of the various stages of the early auditory pathway; e.g., Jewett wave 1 represents the compound action potential of the SGNs, and Jewett waves 2 and 3 reflect signal propagation in the cochlear nucleus (Melcher et al, 1996). Curiously, despite the morphological and physiological deficiencies found at the synapses of endophilin-deficient IHCs, ABR thresholds and amplitudes were comparable between Wt, 1-SKO and 1/3 DKO mice (Fig EV2; note that 1/2-DKO and TKO mice could not be tested due to their premature lethality).

In summary, our combined functional and morphological data indicate a role of endophilins in promoting the abundance of $\mathrm{Ca}_{\mathrm{V}} 1.3$ $\mathrm{Ca}^{2+}$ channels at IHC AZs. In addition, our data suggest that endophilins are required for efficient SV replenishment to IHC AZs as required for sustained exocytosis.

\section{Endophilin-A1 interacts with otoferlin, a key player in hair cell exocytosis}

Our patch-clamp recordings implied that the reduced $\mathrm{Ca}^{2+}$ currents seen in endophilin mutants cannot fully account for the deficits in SV replenishment, and hence, we focused our search on putative presynaptic effector molecules that may be regulated by endophilins. One such potential candidate is the multi- $\mathrm{C}_{2}$ domain protein otoferlin - a key player that is essentially required for IHC exocytosis (Roux et al, 2006; Pangrsic et al, 2010; Vogl et al, 2016). Otoferlin has previously been shown to interact with endocytic adaptor protein AP-2 (Duncker et al, 2013; Jung et al, 2015), and this interaction might serve the clearance of release sites after SV fusion (Jung et al, 2015), therefore placing otoferlin in the same subcellular framework where endophilins are thought to operate. We characterized otoferlin levels and its subcellular distribution in endophilin mutant IHCs using semi-quantitative immunohistochemistry (Fig 5). Interestingly, our analysis revealed a reduction of overall IHC otoferlin immunofluorescence intensity by $28.4 \pm 3.5 \%$ in $1 / 3$-DKOs (Fig 5A-C; unpaired $t$-test, $t=6.71, P<0.0001 \mathrm{Wt}$ versus $1 / 3$-DKO) that however was much less pronounced than in IHCs of AP- $2 \mu$ mutants (by approximately 70\%) (Jung et al, 2015). Furthermore, when assessing the subcellular distribution pattern of otoferlin in mutant IHCs via line profile analysis along the longitudinal IHC axis, we found comparable patterns of otoferlin distribution, but a reduction in the overall signal intensity in $1 / 3$ DKO IHCs (Fig 5C). In contrast, the fluorescence intensity of myosin 6, previously characterized as regulator of IHC maturation and interaction partner of otoferlin (Roux et al, 2009) as well as of AP-2, seemed unchanged in response to the disruption of endophilins (Fig EV3 and Appendix Fig S1, respectively).

In light of the reduction of otoferlin even in the partial absence of endophilin, we aimed to better understand the relation of these proteins. Thus, we tested whether otoferlin interacts with endophilin-A1 by immunoprecipitation. An interaction between otoferlin and endophilin-A1 in vitro was revealed by specific enrichment of endophilin in anti-GFP immunoprecipitates from HeLa cells expressing endophilin-A1-mRFP and EGFP-otoferlin (Fig 5D and $\mathrm{D}^{\prime}$ ). Notably, using an alternative experimental approach, bead-coupled EGFP-otoferlin was able to bind highly purified endophilin-A1 (Fig $5 \mathrm{E}$ and $\mathrm{E}^{\prime}$ ), thereby suggesting an interaction of endophilin-A1 and otoferlin in both systems. In IHCs, such an interaction might aid the coupling of exocytosis and endocytosis.

\section{Endophilin is involved in endocytic membrane retrieval in IHCs}

Next, we performed $C_{m}$ measurements to study whether endophilin deficiency alters endocytic membrane retrieval following 
A
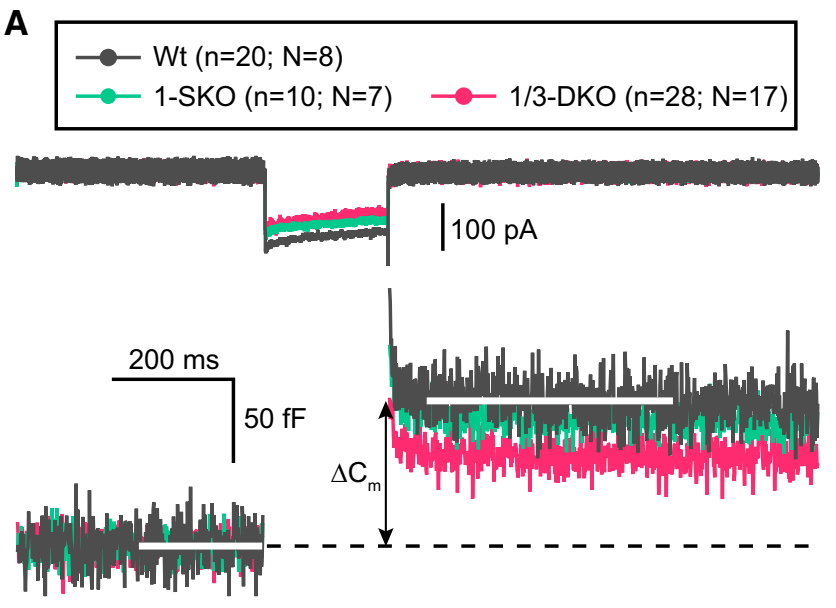

\section{B}

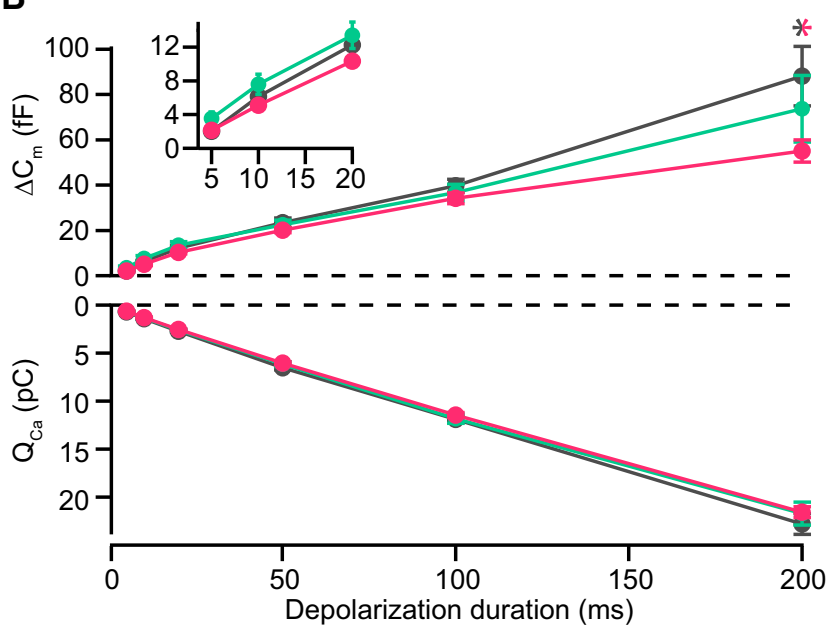

C

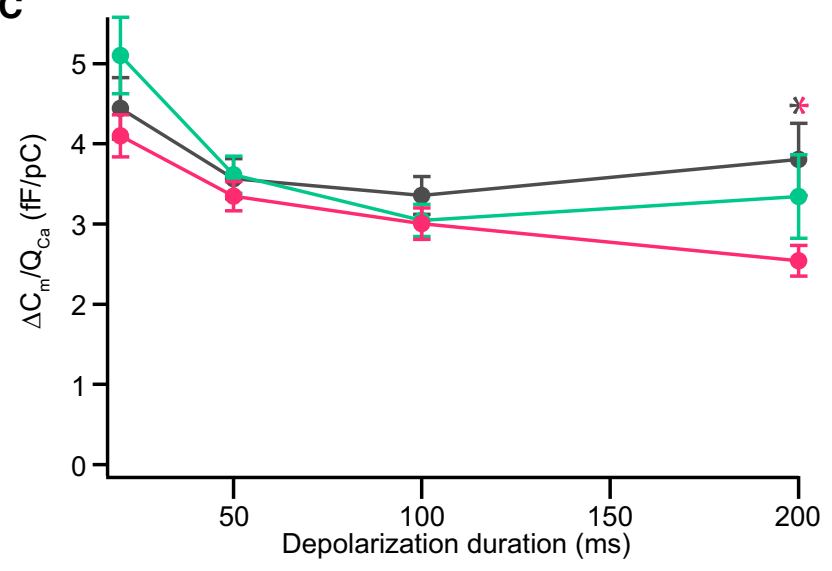

depolarization-induced exocytosis in IHCs after hearing onset. We employed short and long step depolarizations to $-14 \mathrm{mV}$ to trigger different amounts of exocytosis (Fig 6). In IHCs, short depolarizations (20 ms, recruiting the RRP) predominantly result in a slow, near linear post-stimulus $\mathrm{C}_{\mathrm{m}}$ decline back to baseline, which we assume to reflect CME (Neef et al, 2014). Long depolarizations (200 ms) - turning over in excess of 3-4 times the equivalent of the $\mathrm{RRP}$-additionally evoke an exponentially decaying $\mathrm{C}_{\mathrm{m}}$ component that likely involves bulk retrieval (Neef et al, 2014). In these
Figure 4. Sustained exocytosis is impaired in endophilin-deficient IHCs.

A Representative $\mathrm{Ca}^{2+}$ currents (upper panel) and exocytic membrane capacitance increments $\left(\Delta C_{m}\right.$; lower panel) in response to a $200-\mathrm{ms}$ depolarizing step to $-14 \mathrm{mV}$.

B Exocytic $\Delta \mathrm{C}_{\mathrm{m}}$ (top) and corresponding $\mathrm{Ca}^{2+}$ current integrals $\mathrm{Q}_{\mathrm{Ca}}$ (bottom) of $\mathrm{Wt}$ and endophilin-deficient IHCs in response to voltage steps from -84 to $-14 \mathrm{mV}$ of variable stimulus duration (5-200 ms). In 1/3-DKO IHCs, our data indicate reduced sustained exocytosis to a strong depolarization (200 ms; ${ }^{*} P=0.0470, \mathrm{~K}-\mathrm{W}$ with post hoc Dunn's test; please also refer to Appendix Tables S5 and S6 for detailed statistical analysis). Inset: Initial $\Delta C_{m}$ showed comparable RRP exocytosis in endophilin-deficient IHCs.

C Ca ${ }^{2+}$ efficiency to drive exocytosis was reduced for strong depolarizations (200 ms) in 1/3-DKO IHCs ( ${ }^{*} P=0.0323, \mathrm{~K}-\mathrm{W}$ with post hoc Dunn's test; please also refer to Appendix Table $\mathrm{S7}$ for detailed statistical analysis).

Data information: For panels (A-C), the numbers of replicates were Wt $n=20$ / $N=8 ; 1-S K O n=10 / N=7 ; 1 / 3-D K O n=28 / N=17$. Data represent averages \pm SEM.

experiments, we also included 1/2-DKO IHCs into the analysis of the endocytic membrane retrieval as a prominent role of endophilin-A2 in scission and SV reformation had been reported previously (Renard et al, 2015). After a 20-ms depolarization, the endocytic $\mathrm{C}_{\mathrm{m}}$ decline was significantly slowed in IHCs of both $1 / 2$ DKO and 1/3-DKO (Fig 6A, C and D). Here, the mean slope of a line fit to the endocytic $C_{m}$ decline amounted to $-0.51 \mathrm{fF} / \mathrm{s}$ and $-0.50 \mathrm{fF} / \mathrm{s}$ for $1 / 2$-DKO and 1/3-DKO IHCs, respectively, compared to $-0.84 \mathrm{fF} / \mathrm{s}$ in Wt IHCs (Fig 6D; one-way ANOVA, $F(2$, $35)=5.87, P=0.0063$; post hoc Tukey's test: $P=0.0144$ for $1 / 2$ DKO versus $\mathrm{Wt}$ and $P=0.0144$ for $1 / 3-\mathrm{DKO}$ versus $\mathrm{Wt}$ ). Moreover, there was a trend toward a delayed $C_{m}$ return to baseline for IHCs of both mutants that did not reach statistical significance (Fig 6C, one-way ANOVA, $F(2,35)=1.67, P=0.2025$ ). A trend toward slower linear $C_{m}$ decline was also observed in the responses to a 200-ms depolarization (following the exponential phase of retrieval; Fig 6B and D; individual statistics for all endocytosis parameters can be found in Appendix Table S8). These data suggest a reduced rate of membrane retrieval by CME in the absence of endophilins-A1/2 or endophilins-A1/3 in IHCs. In addition, we found a trend toward a smaller and slower exponential phase of $\mathrm{C}_{\mathrm{m}}$ decline in 1/2-DKO and 1/3-DKO IHCs following $200 \mathrm{~ms}$ of depolarization, which however did not reach statistical significance (Fig 6E and F; yet, these data reach statistical significance, if statistical outliers are excluded for the time constant, KWS $=8.52$ and $P=0.0141$ ). In conclusion, our $C_{m}$ measurements indicate a role of endophilins particularly in the slow component of IHC endocytic membrane retrieval, likely reflecting CME.

\section{Endophilin deficiency impairs vesicle uncoating and synaptic vesicle reformation}

In order to further characterize the deficits in endocytosis revealed by electrophysiology, we performed extensive morphological analyses using electron microscopy and electron tomography. Consistent with the immunohistochemical data, the general morphology and plasma membrane anchoring of synaptic ribbons at presynaptic AZs of all genotypes appeared normal (Fig 8A).

In a first step, we used random ultrathin sections to explore the abundance of small, clear, and uncoated vesicles (diameter $<70 \mathrm{~nm}$, likely representing SVs), endosome-like vacuoles (ELVs), 

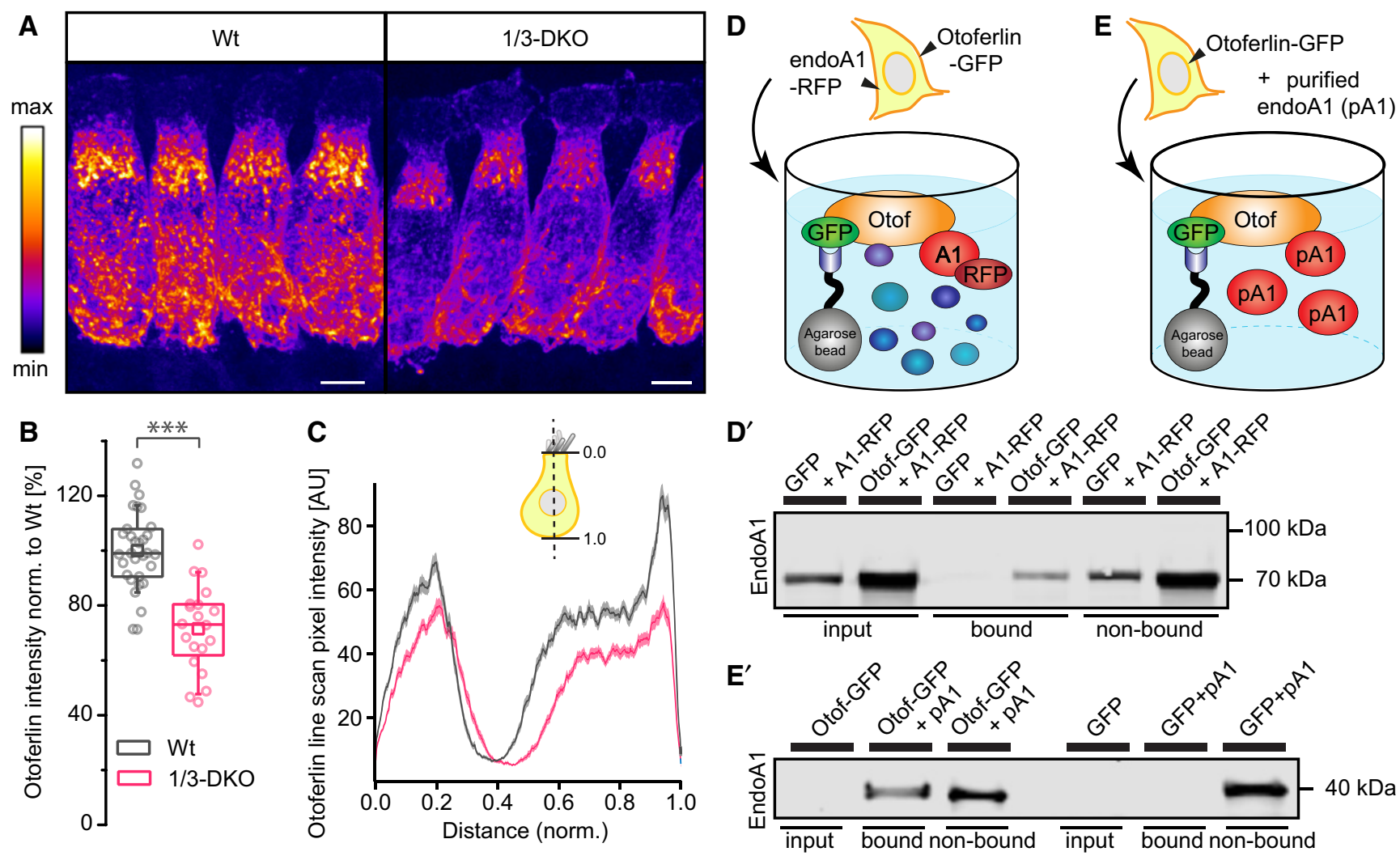

Figure 5. Disruption of endophilins causes a reduction of IHC otoferlin levels.

A Confocal maximum projections of otoferlin-immunolabeled IHCs from p15 Wt and 1/3-DKO mice illustrated with an intensity-coded lookup table where brighter colors indicate higher intensity. Scale bars: $5 \mu \mathrm{m}$.

B Otoferlin levels were reduced by approx. $28.4 \pm 3.5 \%$ in 1/3-DKO IHCs compared to Wt IHCs (relative intensities normalized to Wt $t_{\text {avg }}$; Wt $n=31$ images (229 IHCs)/ $N=5$ organs of Corti (three animals); $1 / 3-D K O n=20$ images (196 IHCS) $/ N=5$ organs of Corti (four animals); unpaired Student's $t$-test; ${ }^{* \star \star} P<0.001$; box plots illustrate the median with the interquartile range, whiskers indicate $10-90 \%$ of data points, and the squares present the respective mean value).

C Normalized otoferlin intensity line profiles through single IHCS of the respective genotypes at a longitudinal central plane through the nucleus from apical (0.0) to basal (1.0) revealed similar distribution patterns of otoferlin in IHCS of Wt and 1/3-DKO, but an overall reduction of otoferlin levels throughout the entire cell in 1/3DKOs. Traces indicate the mean pixel intensity \pm SEM; Wt $n=123 / \mathrm{N}=3 ; 1 / 3-\mathrm{DKO} n=124 / \mathrm{N}=3$.

$D$, E Otoferlin interacts with endophilin-A1. (D, D') Interaction of otoferlin and endophilin-A1 detected by co-IP in HeLa cells co-expressing GFP-otoferlin and RFPendophilin-A1. Otoferlin-GFP was immunoprecipitated (IP) by GFP-Trap beads, and blots were probed with a KO-validated anti-endophilin-A1 antibody. (E, E') Exogenously overexpressed GFP-otoferlin was immunoprecipitated using GFP-Trap beads and incubated with purified endophilin-A1 (pA1). IP was then followed by immunoblotting with an anti-endophilin-A1 antibody.

Source data are available online for this figure.

and clathrin-coated membrane profiles within a radius of $1 \mu \mathrm{m}$ from the synaptic ribbon (Fig 7A). For this analysis, we excluded the SVs directly adjacent to the presynaptic ribbon and considered them in a separate analysis (see below). The total number of small uncoated cytosolic vesicles was elevated in 1-SKO IHCs, but reduced in 1/2-DKO IHCs, while 1/3-DKOs displayed unaltered vesicle counts compared to Wt (Fig 7B; KWS $=130, P<0.0001$; post hoc Dunn's results can be found in Appendix Table S9). Additionally, the area covered by ELVs was increased in 1/2-DKO and 1/3-DKO (Fig 7C; KWS $=70.17, P<0.0001$; post hoc Dunn's results can be found in Appendix Table S9), suggesting compromised reformation of SVs from ELVs in these genotypes. Quantification of clathrin-coated profiles included the following: (i) coated vesicles with diameters $<70 \mathrm{~nm}$, (ii) fully coated vacuoles with diameters $>70 \mathrm{~nm}$, (iii) coated pits at the plasma membrane, and (iv) coated pits budding from ELVs. We observed an overall significantly increased number of coated structures in IHCs of 1SKOs, and, even more prevalently, in 1/3-DKOs and 1/2-DKO compared to Wt (Fig 7D; KWS $=66.1, P<0.0001$ ). While 1 -SKO AZs exhibited more coated vesicles, numbers of coated vacuoles and coated pits budding from the plasma membrane were increased at AZs of both DKOs (Fig 7D'; coated SVs: KWS = 27.13, $P<0.0001$; coated vacuoles: KWS $=33, P<0.0001$; coated pits: KWS $=79.67, P<0.0001$; coated pits at ELVs: $K W S=6.503$, $P=0.0895$; post hoc Dunn's results for all data sets can be found in Appendix Table S9). These data point towards impaired SV uncoating, taking effect already in 1-SKOs as well as additional deficits in fission and/or membrane bending in $1 / 2$-DKOs and $1 / 3$ DKOs during CME.

Secondly, we analyzed small vesicles in direct ribbon proximity (diameters $<70 \mathrm{~nm}$, clear, and uncoated), hereafter categorized as SVs, using electron microscopy of random ultrathin sections 
A
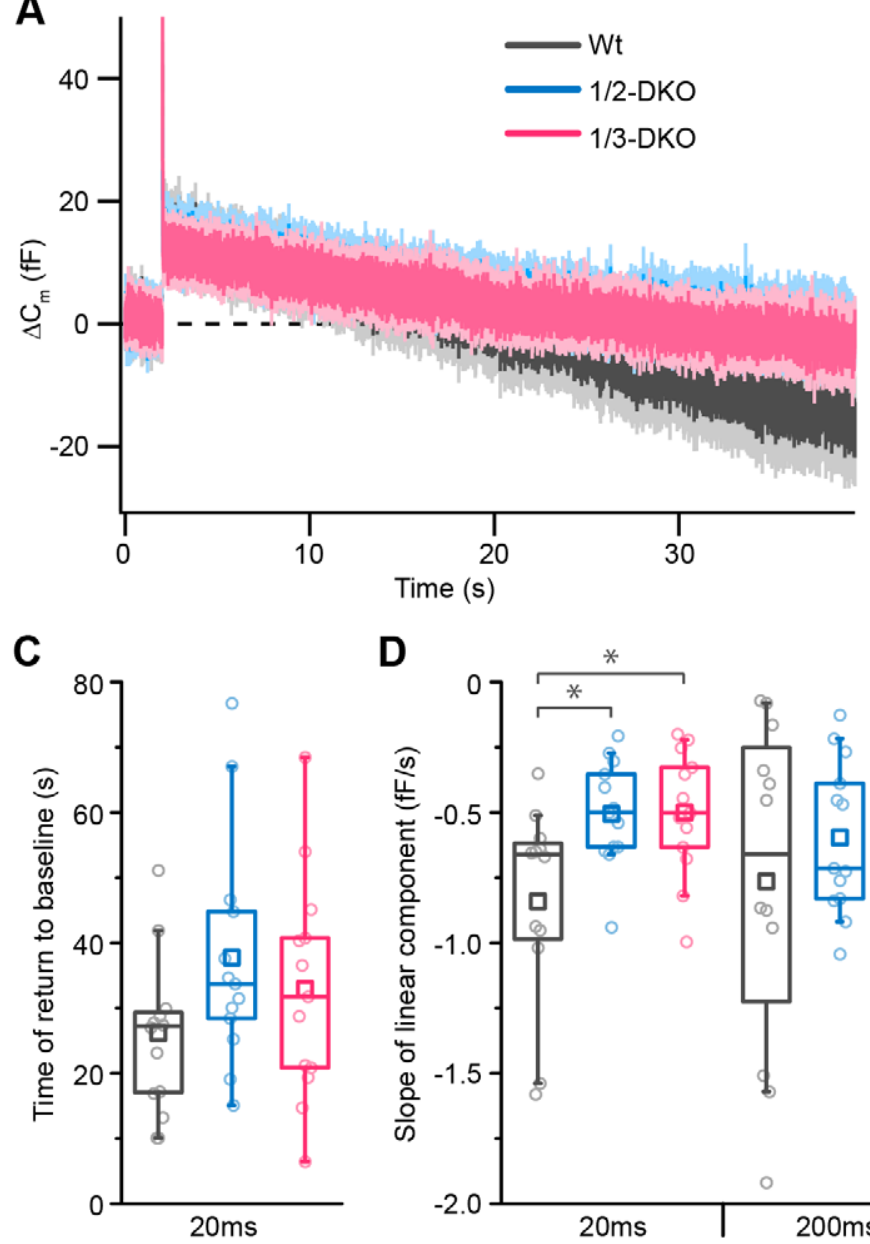

D

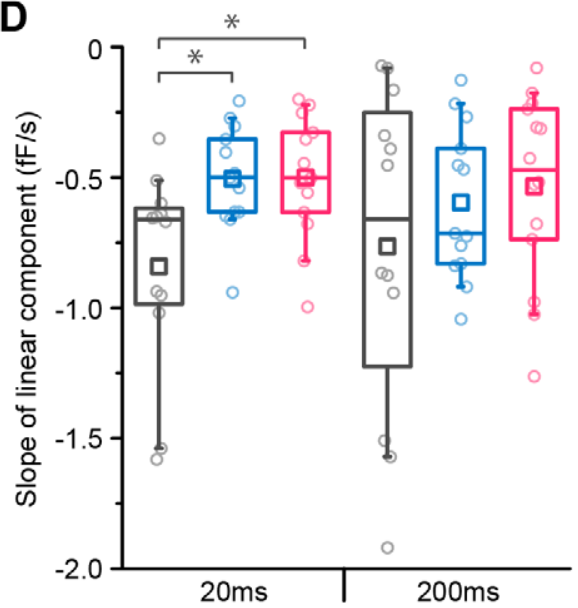

B

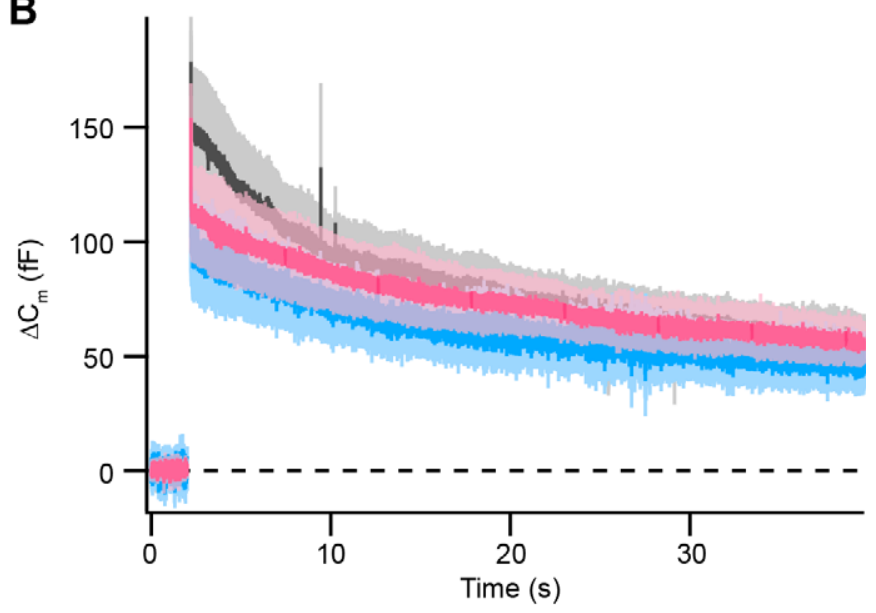

E

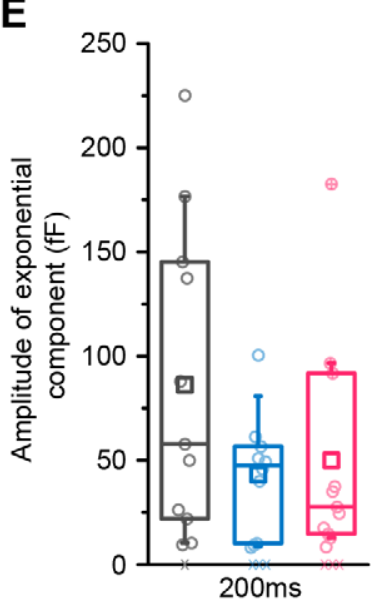

$\mathbf{F}$

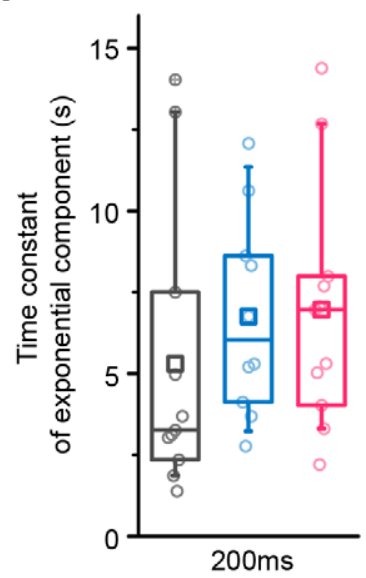

Figure 6. Slowed endocytic membrane retrieval in endophilin-deficient IHCs.

A, B Endocytosis was assessed by determining the decrease in $C_{m}$ during 20 s post-depolarization in perforated patch-clamp recordings from $\mathrm{Wt}$, $1 / 2-\mathrm{DKO}$ and $1 / 3-\mathrm{DKO}$ IHCS. The two kinetic components are presented as average $\Delta \mathrm{C}_{\mathrm{m}}$ recordings after (A) $20 \mathrm{~ms}(\mathrm{Wt} n=12 / \mathrm{N}=8 ; 1 / 2-\mathrm{DKO} n=13 / \mathrm{N}=7 ; 1 / 3-\mathrm{DKO} n=13 / \mathrm{N}=10)$ and (B) $200 \mathrm{~ms}(\mathrm{Wt} n=12 / \mathrm{N}=8 ; 1 / 2-\mathrm{DKO} n=13 / \mathrm{N}=6 ; 1 / 3-\mathrm{DKO} n=14 / \mathrm{N}=10)$ depolarization stimuli.

C Average time of return to baseline obtained by fitting a linear function to the $\mathrm{C}_{\mathrm{m}}$ data following a 20-ms depolarization.

D Average slope of the linear component of endocytosis for 20-ms and 200-ms depolarizing pulses, obtained from fitting $C_{m}$ data for $20 \mathrm{~s}$ after cessation of the 20 ms depolarizations or for the last $10 \mathrm{~s}$ of the $C_{m}$ recording for 200 -ms depolarizations $\left({ }^{\star} P<0.05\right.$; one-way ANOVA with post hoc Tukey's or non-parametric K-W with post hoc Dunn's test).

E, F Average amplitude (E) and time constant (F) of the exponential component obtained from fits to $\mathrm{C}_{\mathrm{m}}$ data following $200-\mathrm{ms}$ depolarization $\left(n_{\text {exp fit }}=11\right.$ for Wt, 10 for $1 / 2-D K O$, and 11 for 1/3-DKO; $x$ data points in (E) correspond to IHCs with no exponential component in $C_{m}$ ). $\otimes$ corresponds to statistically identified outliers.

Data information: For panels (A, B), data represent grand averages \pm SEM; for ( $-F)$, box plots illustrate the median with the interquartile range, whiskers indicate 10 $90 \%$ of data points, and the squares present the respective mean value. Please also refer to Appendix Table S8 for detailed statistical analysis.

(Fig $8 \mathrm{~A}-\mathrm{D}$ ). Here, we observed an overall reduction of SVs at the AZs of $1 / 2$-DKO and 1/3-DKO IHCs. We further categorized SVs into ribbon-associated SVs (RA-SVs) and membrane-proximal SVs (MPSVs) (Fig 8B). The number of RA-SVs was significantly reduced in 1/2-DKO and 1/3-DKO IHCs compared to Wt (Fig 8C: one-way ANOVA, $F(3,417)=38.4, P<0.0001$; post hoc Tukey's analysis for all data sets can be found in Appendix Table S11) with the membrane-distal half of the ribbon being affected to a greater extent than the membrane-proximal half (Fig $8 \mathrm{C}^{\prime}: \quad \mathrm{KWS}=82.4$, $P<0.0001$; post hoc Dunn's analysis can be found in Appendix Table S11). Likewise, the number of MP-SVs was reduced in IHCs lacking endophilins-A1/A2 or endophilins-A1/A3 (Fig 8D: one-way ANOVA, $F(3,425)=17.6, P<0.0001$; post hoc Tukey's results for all data sets can be found in Appendix Table S11).

In order to further decipher the distribution of vesicles and ELVs at the ribbon in 3D, we additionally performed 3D electron tomography, which offers an improved axial resolution to revisit our analysis of $1 / 2$-DKO and $1 / 3$-DKO IHCs (Fig $8 \mathrm{E}-\mathrm{J}$ ). Here, the reconstruction of the electron tomograms (Fig 8E, an overview of the analysis parameters and criteria can be found in Fig $8 \mathrm{~F}$ ) corroborated the above notions and revealed a statistically significant reduction of RA-SVs (Fig 8G: one-way ANOVA, $F(2,21)=3.899, P=0.036$; post hoc Tukey's test: $P=0.023$ for $1 / 2$-DKO versus $W t$ and $P=0.14$ for 1/3-DKO versus Wt) and of MP-SVs (Fig 8H; one-way ANOVA, 

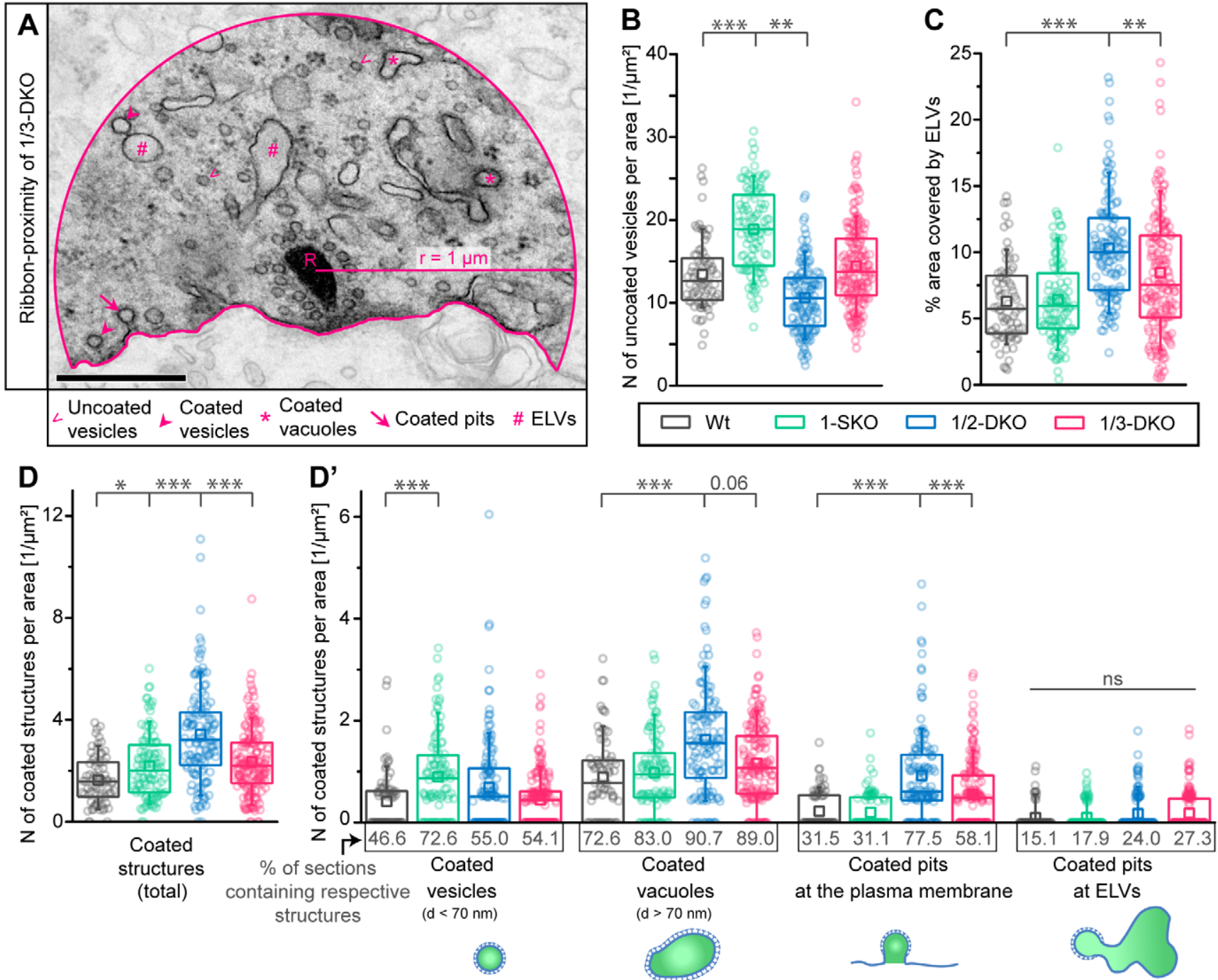

Figure 7. Decreased number of small vesicles, accumulations of coated structures and ELVs at IHC synapses of endophilin mutants.

A Representative electron micrograph illustrating the region of interest for analysis within a radius of $r=1 \mu \mathrm{m}$ from the ribbon center (exemplary for a 1/3-DKO specimen). Graphical aids indicate small vesicles, ELVs, and different subpopulations of coated structures quantified in (B-D). Scale bar: $500 \mathrm{~nm}$.

B While an increased number of small uncoated vesicles could be observed at 1-SKO AZs, reduced numbers of uncoated vesicles were present in a $1 \mu \mathrm{m}$ radius around the ribbon of 1/2-DKOs. RA-SVs and MP-SVs were excluded from this quantification.

C Accumulations of ELVs occurred in 1/2-DKO and 1/3-DKO IHCS, as measured by the relative cumulative ELV area per section.

D Increased overall number of coated structures in endophilin mutants. ( $\mathrm{D}^{\prime}$ ) Prominent accumulations of coated pits could be observed in 1/2-DKO and 1/3-DKO IHCS alongside a shift toward more coated vacuoles (d $>70 \mathrm{~nm}$ ) in 1/2-DKO IHCS and 1/3-DKO IHCS.

Data information: For panels (B-D), the following numbers of replicates were used: Wt $n=72 / \mathrm{N}=2 ; 1$-SKO $n=106 / \mathrm{N}=3 ; 1 / 2-\mathrm{DKO} n=129 / \mathrm{N}=3 ; 1 / 3$-DKO $n=172 /$ $N=6$. Box plots illustrate the median with the interquartile range, whiskers indicate 10-90\% of data points, and the squares present the respective mean value; $\mathrm{K}-\mathrm{W}$ followed by Dunn's test; ${ }^{*} P<0.05$; ${ }^{* *} P<0.01$; ${ }^{* \star *} P<0.001$. Please also refer to Appendix Tables $\$ 9$ and $\$ 10$ for detailed statistical analysis.

$F(2,21)=4.912, P=0.0178$; post hoc Tukey's test: $P=0.014$ for $1 /$ 2 -DKO versus $\mathrm{Wt}$ and $P=0.051$ for $1 / 3$-DKO versus $\mathrm{Wt}$ ) in $1 / 2$-DKO IHCs. The fraction of RA-SVs of the total count of SVs at the AZ was not significantly affected (one-way ANOVA; $F(2,21)=0.4416$, $P=0.6488$; see Appendix Fig S2B). Studying SV diameters with the greater precision of tomography, we found larger SVs at $1 / 2$-DKO AZs, but not at 1/3-DKO AZs (Fig 8I; one-way ANOVA, $F(2,21)=11.59, P=0.0004$; post hoc Tukey's test: $P=0.0215$ for $1 / 2$-DKO versus $\mathrm{Wt}$; $P=0.176$ for $1 / 3$-DKO versus $\mathrm{Wt}$; $P=0.0003$ for $1 / 2$-DKO versus $1 / 3$-DKO). Moreover, tomograms showed an increased abundance of ELVs at 1/2-DKO AZs and a non-significant trend for more ELVs at 1/3-DKO AZs (Fig 8J; one-way ANOVA, $F(2,21)=11.59, P=0.0004$; post hoc Tukey's test: $P=0.015$ for $1 /$ 2 -DKO versus $\mathrm{Wt}$; $P=0.136$ for $1 / 3$-DKO versus $\mathrm{Wt}$ ).

Finally, we examined the effects of potassium stimulation on the ultrastructure of endophilin-deficient IHCs. In these experiments, we subjected acutely dissected 1/2-DKOs-the genotype with the most perturbed presynaptic morphology - and Wt organs of Corti to a stimulatory solution ( $15 \mathrm{~min}, 50 \mathrm{mM} \mathrm{KCl}, 5 \mathrm{mM} \mathrm{CaCl}_{2}$ ) prior to immediate chemical fixation (Fig 9A). Analogous to the 

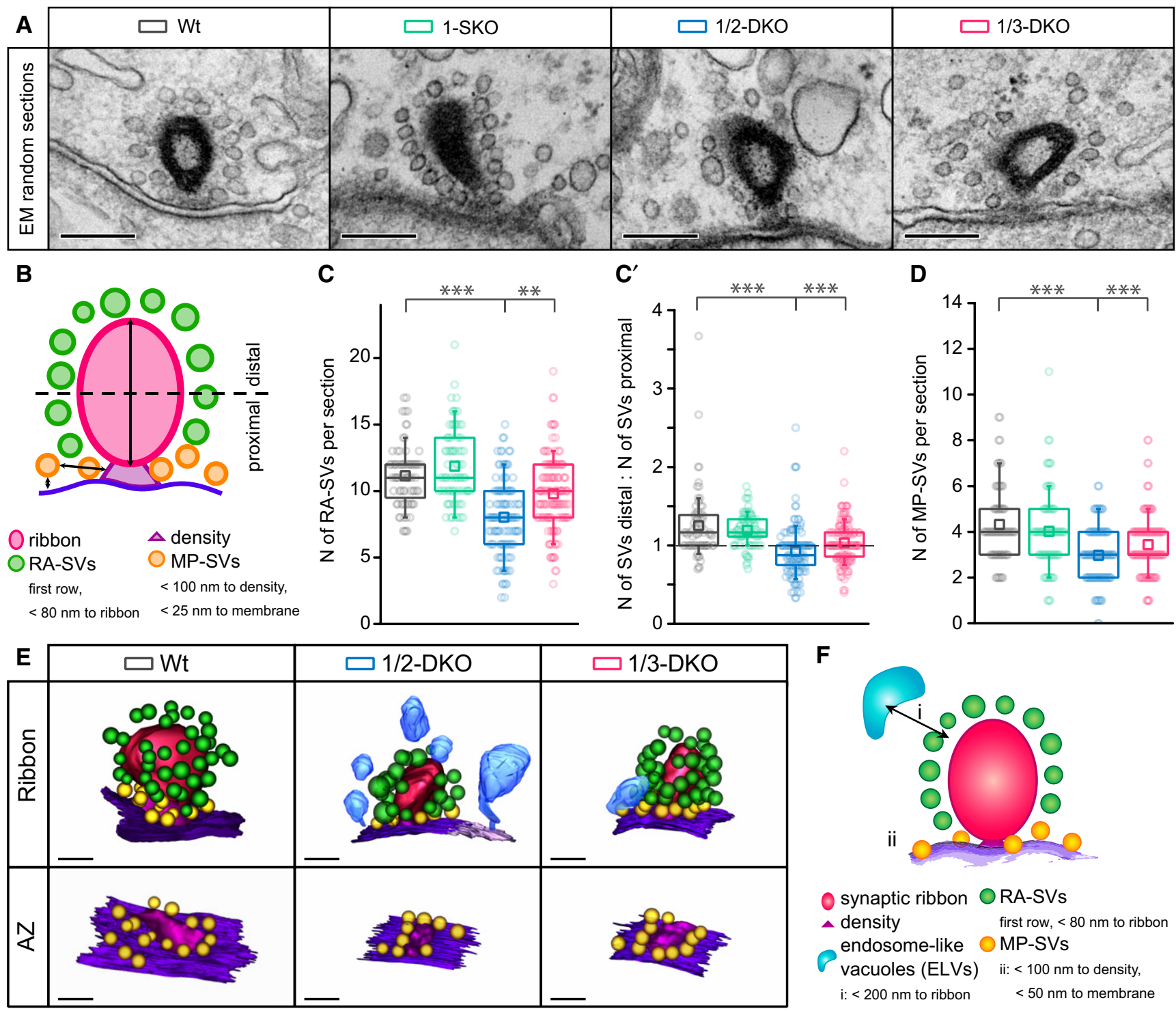

F
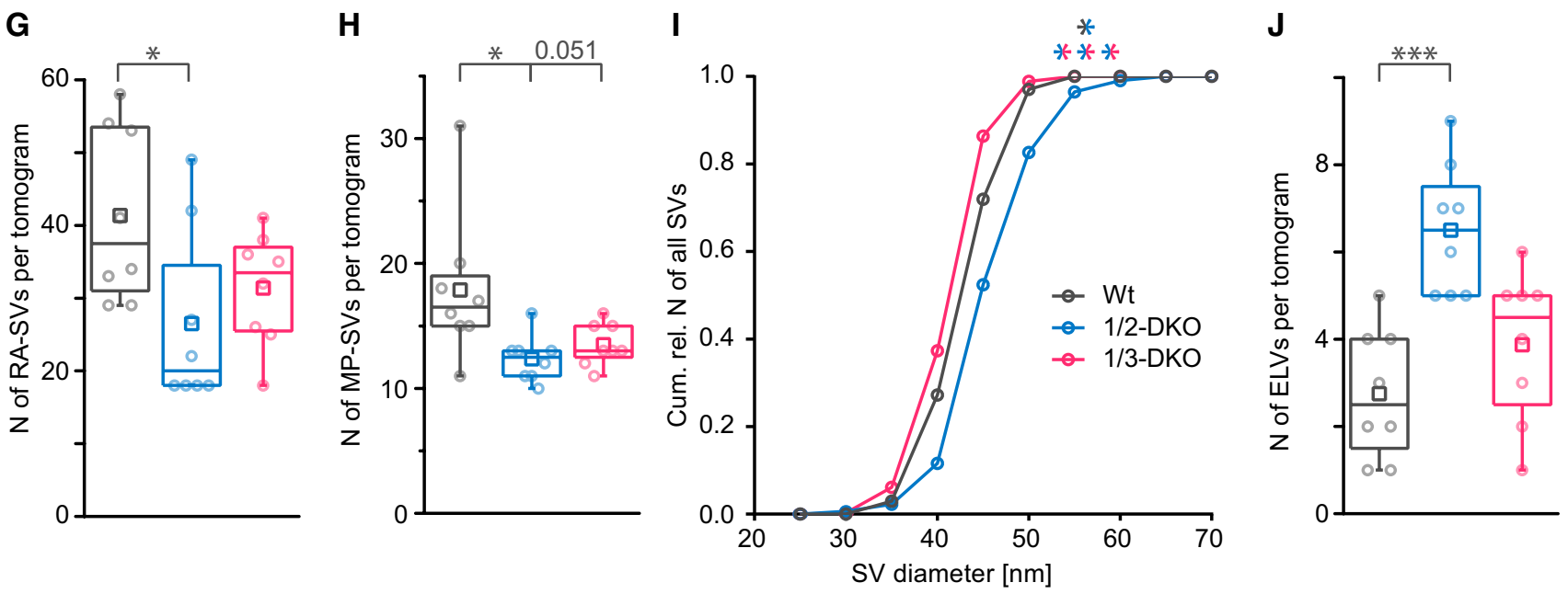

Figure 8. 
Figure 8. Absence of endophilins causes changes in SV number and distribution at IHC ribbon synapses.

A Representative electron micrographs of random ultrathin sections from IHC ribbon synapses of p15 mutants lacking different endophilin-A alleles. Scale bars: $200 \mathrm{~nm}$.

B Schematic illustration depicting the analysis criteria for random section electron micrographs (not drawn to scale). Synaptic vesicles (SVs) were categorized as ribbon-associated SVs (RA-SVs, green) or membrane-proximal SVs (MP-SVs, yellow).

C, D Reduced numbers of RA-SVs (C) and of MP-SVs (D) in IHCS of endophilin mutants with the strongest reduction observed in 1/2-DKO mice. One-way ANOVA followed by post hoc Tukey's test; ${ }^{* \star} P<0.01 ;{ }^{* \star *} P<0.001$

$\mathrm{C}^{\prime} \quad$ Reduced ratio of RA-SVs in the distal half of the ribbon over RA-SVs in its proximal half in 1/2-DKO and 1/3-DKO IHCS. K-W followed by Dunn's test; $\star \star \star P<0.001$.

E Representative 3D reconstructions of EM tomograms of 250-nm sections from Wt and endophilin DKO mutants. Please note the accumulations of ELVs in IHCs of endophilin 1/2-DKOs. Analysis was performed on eight tomograms from two animals per genotype. Scale bars: $100 \mathrm{~nm}$.

F Schematic illustration depicting the analysis criteria for tomograms (not drawn to scale). SVs were categorized as RA-SVs (green) and as MP-SVs (yellow). ELVs (light blue) were counted if the smallest distance between ELV and ribbon was $<200 \mathrm{~nm}$.

G Significantly reduced numbers of RA-SVs in 1/2-DKO IHCS (one-way ANOVA followed by post hoc Tukey's test; ${ }^{\star} P=0.0229$ ).

$\mathrm{H} \quad$ Significantly reduced numbers of MP-SVs in $1 / 2-\mathrm{DKO}$ and a strong trend $(P=0.0511$ ) toward less MP-SVs in $1 / 3-\mathrm{DKO}$ IHCS (one-way ANOVA followed by post hoc Tukey's test; $\left.{ }^{*} P=0.0138\right)$

Increased SV diameter in 1/2-DKOs, but unchanged SV diameter in 1/3-DKOs (one-way ANOVA followed by post hoc Tukey's test; ${ }^{\star} P=0.0154$ ).

J Accumulation of ELVs with a minimal distance to the ribbon of less than $200 \mathrm{~nm}$ in 1/2-DKOs (one-way ANOVA followed by post hoc Tukey's test; ${ }^{* \star \star} P=0.0002$ ).

Data information: Analysis was performed on random sections of ribbon-occupied AZs derived from several IHCS per genotype; the following numbers of replicates were used: Wt $n=78$ sections from $N=2$ animals; 1 -SKO $n=95, N=3 ; 1 / 2$-DKO $n=135, N=3 ; 1 / 3$-DKO $n=176, N=6$. Box plots illustrate the median with the interquartile range, whiskers indicate $10-90 \%$ of data points, and the squares present the respective mean value. For panels (C-E), please also refer to Appendix Tables S11 and S12 for detailed statistical analysis.

unstimulated probes, we compared SV pools in direct vicinity of the ribbon. Here, both, RA- and MP-SV numbers were significantly lower in stimulated 1/2-DKO compared to stimulated Wt (Fig 9B and C; Mann-Whitney test, for RA-SVs: $U=368, P<0.0001$; for MP-SVs: $U=783, P=0.0438$ ). Moreover, all findings made within the analyzed $1 \mu \mathrm{m}$ perimeter of the ribbon of unstimulated $1 / 2$-DKO IHCs compared to unstimulated Wt IHCs were also present in the stimulated IHCs of 1/2-DKO (compared to stimulated Wt IHCs), but to a greater extent. We found (i) reduced numbers of small uncoated cytosolic vesicles (Fig 9D; Student's $t$-test, $t=4.67$; $P<0.0001$ ) and (ii) vast accumulations of ELVs (Fig 9E; Mann-Whitney test, $U=264 ; P<0.0001)$. The formation of coated pits at ELVs was more frequently seen in stimulated $1 / 2$-DKO than in stimulated $\mathrm{Wt}$ or unstimulated samples (Fig 9A and $\mathrm{F}^{\prime}$; Mann-Whitney test, $U=623, P=0.001$; see also Appendix Table S13 for the comparisons of other coated structures), again pointing toward a function of endophilin in clathrin-dependent SV reformation from ELVs.

In summary, our electrophysiological and ultrastructural analyses indicate that loss of endophilins from IHCs impaired, but did not abolish, presynaptic endocytosis. Fission and uncoating of clathrincoated membranous structures, e.g., processes such as clathrindependent SV reformation, seem to be facilitated by-but do not essentially require - endophilins in IHCs.

\section{Discussion}

The presented morphological and physiological analysis of IHC ribbon synapses lacking endophilins provides novel insights into the molecular regulation of transmitter release and membrane retrieval in IHCs. Based on our findings, we propose that the main functions of the endophilin family at IHC synapses are to promote (i) the organization of $\mathrm{Ca}^{2+}$ channel clusters and presynaptic $\mathrm{Ca}^{2+}$ influx, (ii) replenishment of SVs to the release sites, (iii) coupling of exocytosis and endocytosis, probably via interaction with otoferlin, (iv) endocytic membrane retrieval, and (v) SV reformation from clathrincoated structures (summarized in the cartoon in Fig 10).

\section{Endophilin-A1 positively regulates the abundance and modulates the function of Cav1.3 channels at IHC synapses}

While endophilins are indispensable for life, they do not seem to be required for the development and survival of the organ of Corti, even though single-cell RT-PCR indicated the expression of all three endophilins in IHCs. Given the perinatal lethality of endophilin TKO mice, we analyzed their IHCs in organotypic cultures, which did not show obvious developmental or gross morphological deficits. They did, however, show a reduction of depolarization-evoked $\mathrm{Ca}^{2+}$ currents and enhanced inactivation kinetics (in the TKO), features that could partly also be recapitulated in the viable 1 -SKOs and $1 / 3$ DKO mice after hearing onset. Moreover, STED microscopy of $\mathrm{Ca}_{\mathrm{V}} 1.3$ immunofluorescence indicated a reduction in $\mathrm{Ca}^{2+}$ channel cluster size at the presynaptic AZ membrane of endophilin mutants. These observations provide insights into the functional consequences of the interaction of endophilins with $\mathrm{Ca}^{2+}$ channels that had previously been reported by biochemistry (Chen et al, 2003). To our knowledge, this is the first report showing such a positive regulation of $\mathrm{Ca}^{2+}$ channel abundance and function (i.e., inhibiting inactivation) by endophilins. The $\mathrm{Ca}^{2+}$ current inactivation phenotype we observed in organotypically cultured IHCs of TKO mice appeared to be compensated in the other mutants studied-likely by expression of the remaining endophilins-and hence seems to require the complete loss of all endophilins to become apparent. Notably, the observed decrease in whole-cell $\mathrm{I}_{\mathrm{Ca}}$ was not accompanied by (i) detachment of synaptic ribbons, (ii) loss of afferent synapses, or (iii) mislocalization of presynaptic $\mathrm{Ca}_{\mathrm{V}} 1.3 \mathrm{Ca}^{2+}$ channels. Indeed, the channels remained tightly clustered in the AZ membrane underneath the normally sized synaptic ribbon, as shown by super-resolution microscopy. Instead, our data reflect a reduced presynaptic abundance of $\mathrm{Ca}_{V} 1.3 \mathrm{Ca}^{2+}$ channels, as demonstrated by the decreased size of presynaptic $\mathrm{Ca}^{2+}$ channel clusters of 1-SKOs and 1/3-DKOs. Future studies should test how endophilins promote a large $\mathrm{Ca}^{2+}$ channel complement at the IHC AZ and whether such positive regulation of $\mathrm{Ca}^{2+}$ channel abundance is found also at other synapses. 

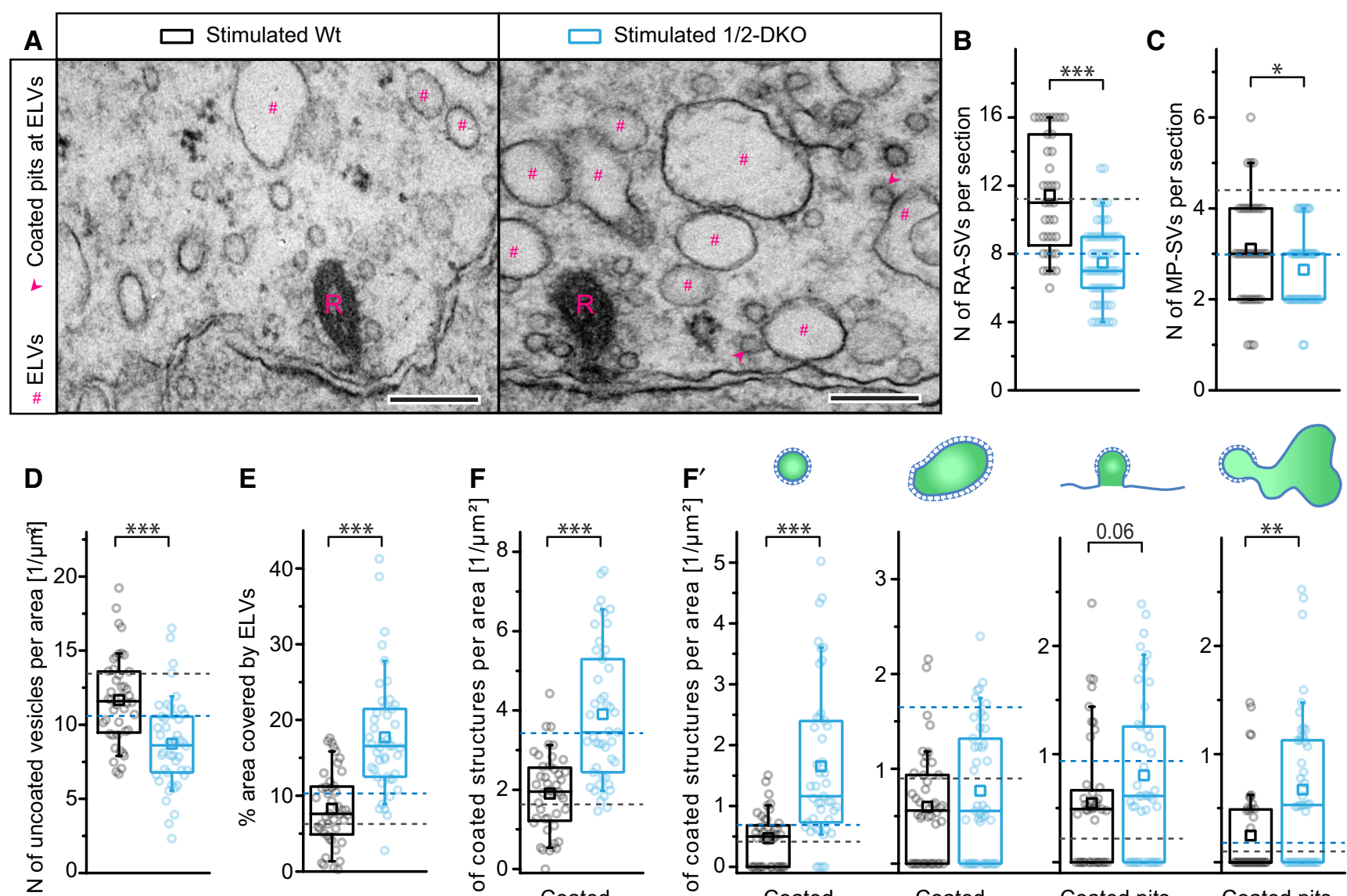

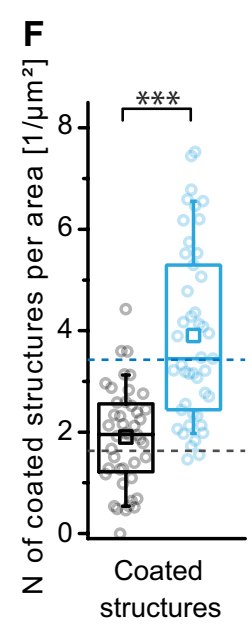

(total)

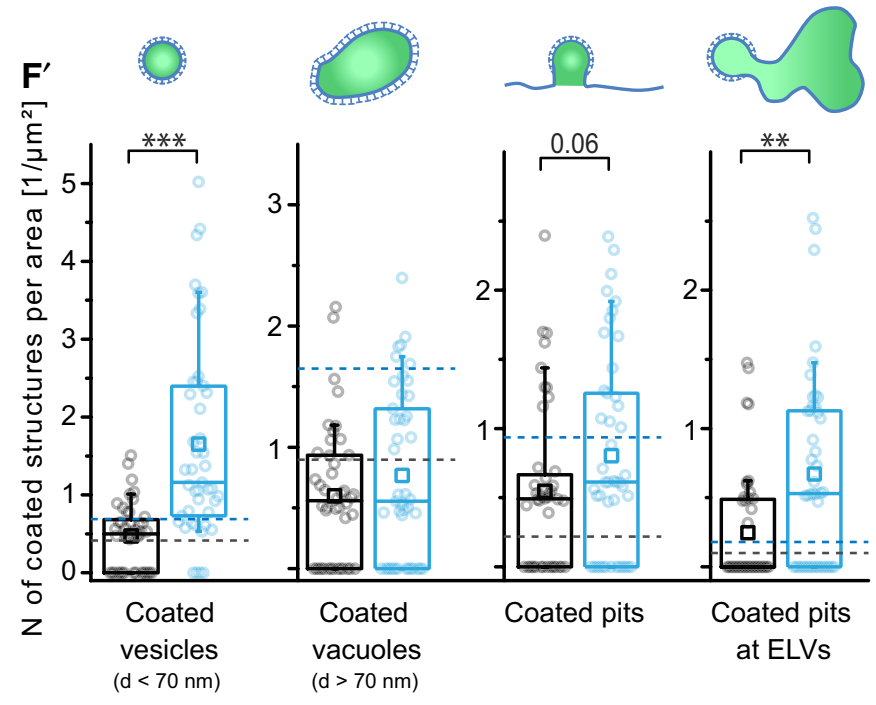

Figure 9. High $\mathrm{K}^{+}$stimulation leads to accumulations of ELVs and an increased occurrence of coated pits at ELVs in endophilin 1/2-DKOs.

A Representative electron micrographs of Wt and 1/2-DKO specimen indicate accumulations of ELVs and high numbers of coated pits forming from ELVs in ribbon

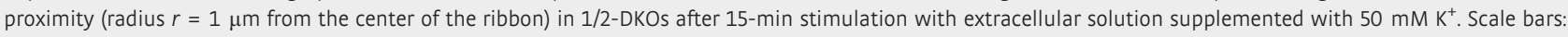
$200 \mathrm{~nm}$.

B Significantly reduced numbers of RA-SVs in IHCS of 1/2-DKO compared to Wt after stimulation, as quantified using random ultrathin sections of ribbon-occupied AZs from several IHCS per genotype. Mann-Whitney test; ${ }^{* *} P<0.001$

C Numbers of MP-SVs were slightly reduced in stimulated 1/2-DKOs compared to stimulated Wt. Mann-Whitney test; ${ }^{*} P<0.05$.

D Numbers of small uncoated vesicles were reduced in a $1 \mu \mathrm{m}$ radius around the ribbon of 1/2-DKOs compared to Wt after stimulation. RA-SVs and MP-SVs were excluded from this quantification. Student's $t$-test; ${ }^{* \star} p<0.001$.

E Stimulated 1/2-DKOs exhibited accumulations of ELVs in comparison with stimulated Wt. Mann-Whitney test; *** $<0.001$

F Increased overall number of coated structures in stimulated endophilin 1/2-DKOs compared to stimulated Wt. (F') Both, coated vesicles and coated pits forming at ELVs, were significantly increased in 1/2-DKOs after stimulation. Mann-Whitney test; ${ }^{\star \star} P<0.01$; ${ }^{* \star *} P<0.001$.

Data information: Stimulated Wt $n=36$ sections from $N=2$ animals; stimulated 1/2-DKO $n=76 / N=3$; dashed lines indicate mean values of the unstimulated conditions from the respective genotypes for reference. Box plots illustrate the median with the interquartile range, whiskers indicate $10-90 \%$ of data points, and the squares present the respective mean value. For panels (B-F), please also refer to Appendix Table S13 for detailed statistical analysis.

\section{Endophilins facilitate endocytic membrane retrieval and reformation of SVs}

Our focus on mature IHCs, moving away from cultured TKO cells, leaves room for functional compensation given that endophilins have previously been shown to exhibit redundancy for several cellular functions (Milosevic et al, 2011; Murdoch et al, 2016). Nonetheless, we found a modest accumulation of clathrin-coated pits (CCPs) in the proximity of IHC ribbon-type AZs, suggesting that even partial absence of endophilins becomes rate-limiting for the fission process at
IHC synapses. Indeed, this hypothesis is strongly supported by our finding of a reduced rate in the slow (linear) component of endocytic membrane retrieval in 1/2-DKO and 1/3-DKO IHCs that has previously been attributed to CME (Neef et al, 2014). In neurons, endophilin is recruited to CCPs prior to membrane fission (Ringstad et al, 1999; Perera et al, 2006; Ferguson et al, 2009; Milosevic et al, 2011; Sundborger et al, 2011), and several other studies have reported an accumulation of CCPs following perturbation of endophilin function (Ringstad et al, 1999; Gad et al, 2000; Schuske et al, 2003; Verstreken et al, 2003; Sundborger et al, 2011). Yet, no significant 


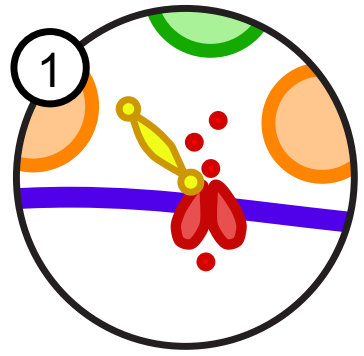

$\mathrm{Ca}_{\mathrm{v}} 1.3$ functional modulation reductions of $\mathrm{Ca}^{2+}$ influx alongside a reduced size but preserved shape of $\mathrm{Ca}_{\vee} 1.3$ clusters

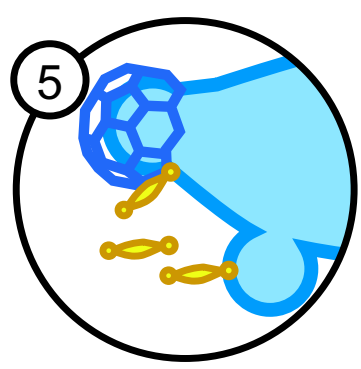

SV formation from ELVs

reduced SV numbers, ELV accumulations and reduction of sustained exocytosis beyond what is expected based on lowered $\mathrm{Ca}^{2+}$ influx 3

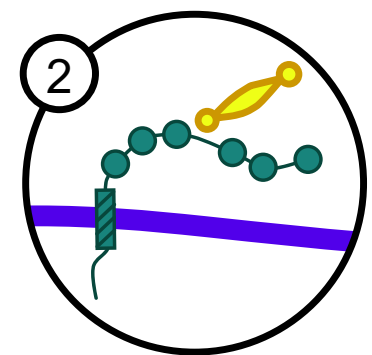

Coupling of exo- and endocytosis direct interaction of endophilin and otoferlin

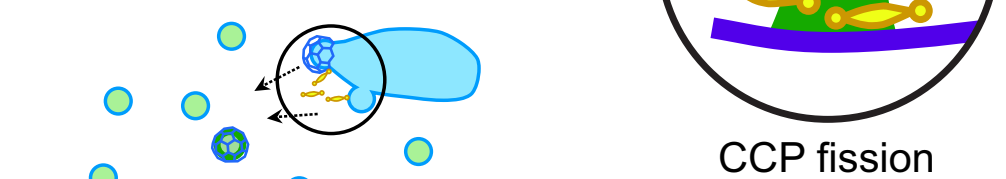

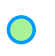

0
O

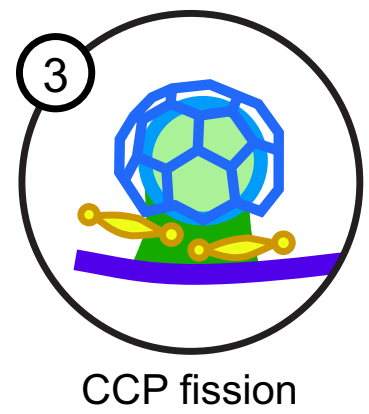

increased numbers of coated pits and slower rates of $\mathrm{IHC}$ endocytic membrane retrieval
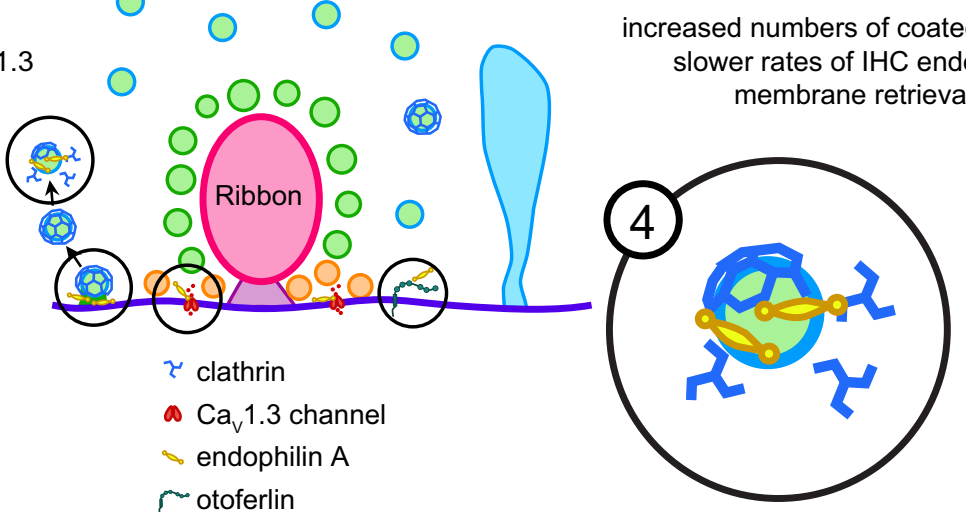

SV uncoating

accumulations of coated structures in proximity of the ribbon

Figure 10. Putative functions of endophilins in IHCs.

All three endophilin-A genes are present in IHCs. We found two novel functions of endophilin-A1: a positive regulation of IHC presynaptic Ca ${ }^{2+}$ influx and Ca ${ }^{2+}$ channel abundance (1) and a physical interaction of endophilin-A1 with the exocytic protein otoferlin that might contribute to exocytosis-endocytosis coupling (2). Moreover, like in neurons, all endophilins in IHCs seem to be involved in fission (3) and uncoating (4) of clathrin-coated organelles supporting CME, and, furthermore, promote SV reformation from endosomal intermediates (5).

accumulation of CCPs was detected at cortical synapses at rest in endophilin 1/2-DKO and TKO mice (Milosevic et al, 2011), suggesting that fission was not rate-limiting in this model. Given that endophilins interact directly with dynamin 1 (Ringstad et al, 1999; Anggono \& Robinson, 2007), they likely promote dynamin's recruitment to the neck of CCPs in IHCs, as they do in neurons (Perera et al, 2006; Ferguson et al, 2009; Milosevic et al, 2011). Furthermore, endophilins have been reported to have a direct role in dynamin-independent scission of tubular membrane necks in vitro (Renard et al, 2015; Simunovic et al, 2017). It is therefore likely that endophilin promotes fission in IHCs via recruitment of dynamins; however, a dynamin-independent role of endophilin in fission remains possible.

Not only the higher numbers of coated pits but also larger coated vacuoles in IHCs of both endophilin DKOs may serve as an indicator for the impaired fission. Alternatively or in addition, endophilin may, as previously reported in invertebrates (Bai et al, 2010), be required for membrane bending prior to the pit formation, thus resulting in larger coated structures. Curiously, the number of coated vesicles was significantly increased in 1-SKO IHCs, but not in 1/2-DKO or 1/3-DKO IHCs, which have an overall much stronger phenotype. Yet, if the numbers of coated vesicles and coated vacuoles are pulled together, one can notice a shift toward larger coated structures in the endophilin 1/2-DKO and 1/3-DKO IHCs. One possible explanation here is that the recruitment of dynamin(s) and/or the fission process may not be rate-limiting in the absence of endophilin-A1 alone. The recruitment of synaptojanin-1 and/or other factors involved in uncoating is already disturbed in synapses of endophilin A1-SKOs, though. This finding is in agreement with Milosevic et al (2011) who reported accumulations of CCVs at murine cortical synapses of $1 / 2$-DKO and TKO mice.

In addition to the increased occurrence of coated pits and coated vesicles in the proximity of IHC ribbon synapses, we observed a 
prominent accumulation of other recycling intermediates with a clathrin coat. Most strikingly in stimulated 1/2-DKO IHCs, coated pits accumulated at ELVs, indicating that fission also displays the rate-limiting step here. Consistent with a role of endophilins in SV reformation, we further found an increased occurrence of ELVs at endophilin 1/2-DKO mutant AZs and a larger area covered by ELVs in both DKO IHCs. Similar accumulation of ELVs can also be found in AP-2-deficient IHCs, which however, unlike endophilin mutants, showed a clear reduction of coated structures in the ribbon's vicinity (Jung et al, 2015). One likely hypothesis is that clathrin recruitment is disturbed in AP-2 mutants, whereas in endophilin mutants, fission and/or uncoating limits endocytic membrane retrieval and SV reformation.

Future studies should address to which extent the impaired SV reformation contributes to the reduction of vesicle replenishment during sustained exocytosis observed in our $\mathrm{C}_{\mathrm{m}}$ recordings in endophilin-deficient IHCs (see below). Importantly, while a prominent reduction in the number of SVs and protein levels of several main SV proteins had been observed at cortical synapses, we detected only mild-albeit statistically significant-alterations in vesicle numbers of endophilin mutants (as reported in Figs 7 and 8). It is not trivial to test the levels and distribution of proteins in this system, in part since IHCs do not use the same molecular machinery as cortical neurons: for example, (i) IHC exocytosis operates without classical neuronal SNAREs including synaptobrevin-2 (Nouvian et al, 2011) and (ii) since various antibodies against SV proteins are found to be non-specific in our hands in IHCs (e.g., synaptojanin-1), thus limiting the tools available to examine SV proteins in IHCs. Nevertheless, while we observed altered levels/distribution of otoferlin and $\mathrm{Ca}^{2+}$ channels, we did not detect obvious differences in the levels and/or distribution of AP-2 or myosin 6, which has previously been reported to be required for ribbon synapse maturation and function (Roux et al, 2009), or AP-2.

\section{Endophilins are involved in SV replenishment at IHC AZs}

Double and triple mutants of endophilins showed reduced sustained exocytosis, likely caused by impaired recruitment of SVs to the release sites of the IHC AZ. It is unlikely that the deficits in sustained exocytosis solely reflect the slowed reformation of SVs (see above), as the overall numbers of small, uncoated vesicles were unaltered in 1/3-DKOs. Here, to our knowledge for the first time, we describe an interaction of endophilin-A1 with otoferlin, a critical component in the processes of synaptic release and SV replenishment in IHCs (Roux et al, 2006; Pangrsic et al, 2010; Vogl et al, 2016). This interaction appears especially relevant in light of the previously described observations. In fact, in mouse mutants suffering from reduced otoferlin levels, such as the otoferlin mutants pachanga (Pangrsic et al, 2010) or otoferlin Ile515Thr (Strenzke et al, 2016), as well as a knockout mouse model for AP- $2 \mu$ (Jung et al, 2015), sustained exocytosis is likewise disturbed.

However, the mechanism at work here seems to be different from what was observed in AP- $2 \mu \mathrm{KO}$. While endophilin-A1, like AP-2, is an interaction partner of otoferlin (Duncker et al, 2013; Jung et al, 2015), lack of endophilin does not cause a drastic decrease in IHC otoferlin levels or alter the subcellular distribution of otoferlin, as seen in AP- $2 \mu$-deficient IHCs. In fact, genetic loss of $\mathrm{AP}-2 \mu$ results in dramatically decreased otoferlin expression and a significant reduction in sustained exocytosis, as well as a profound hearing loss, as assessed by ABR recordings. Importantly, AAVmediated AP- $2 \mu$ rescue succeeded in restoring otoferlin levels of AP$2 \mu$ mutant IHCs to $\sim 50 \%$ of Wt levels, which sufficed to fully restore the observed exocytosis phenotype and drastically improved ABR performance. Based on these findings, we would suspect that the overall reduction of $\sim 25 \%$ of otoferlin levels in endophilin 1/3-DKO should not have any major effects on IHC exocytic performance in the endophilin mutants. Therefore, we propose that the impairment of sustained exocytosis is not due to the reduction of otoferlin levels. The precise mechanism(s) by which endophilins promote replenishment of SVs to the release site remain to be investigated in future studies. The reduced number of RA-SVs at endophilin-deficient AZs might indicate that endophilins are required for efficient resupply of SVs to the ribbon. Given that endophilins are capable of interacting with actin and/or actin-modifying proteins such as intersectin (Ferguson et al, 2009; Soda et al, 2012; Vehlow et al, 2013; Pechstein et al, 2015; Yang et al, 2015), as well as that the F-actin cytoskeleton is important for SV exocytosis in IHCs (Vincent et al, 2015; Guillet et al, 2016), an additional role of endophilin in SV resupply to the ribbon appears likely. Alternatively or in addition, endophilin binding to otoferlin at the release site might facilitate the lateral diffusion of fused vesicular proteolipids (site clearance) as proposed for AP-2 (Jung et al, 2015).

\section{Materials and Methods}

\section{Animals}

All experiments complied with national animal care guidelines and were approved by the University Medical Center Göttingen Board for animal welfare and the animal welfare office of the state of Lower Saxony. Constitutive knockout mice for endophilin-A1 $\left(E 1^{-/-}\right)$, endophilin-A2 $\left(E 2^{-/-}\right)$, endophilin-A3 $\left(\mathrm{E}^{-/-}\right)$, described in Milosevic et al (2011), were employed in two separate breeding schemes: (i) perinatally lethal $\mathrm{E} 1^{-/-} \mathrm{E} 2^{-/}-\mathrm{E} 3^{-/-}$(hereafter dubbed TKO) mice, as well as viable $\mathrm{E} 1^{-/-} \mathrm{E} 2^{+/-} \mathrm{E} 3^{-/-}$and $\mathrm{E} 1^{-/-} \mathrm{E} 2^{+/}$ ${ }^{+} \mathrm{E} 3^{-/-}$mice (hereafter pooled, as we did not find significant differences in IHC physiology and morphology and dubbed 1/3DKOs), were obtained from breeding $\mathrm{E} 1^{-/-} \mathrm{E} 2^{+/-} \mathrm{E} 3^{-/-}$mice and (ii) mating of $\mathrm{E} 1^{-/-} \mathrm{E} 2^{+/-}$mice yielded $\mathrm{E} 1^{-/-} \mathrm{E} 2^{-/-}$mice (hereafter dubbed 1/2-DKOs), and $\mathrm{E} 1^{-/-} \mathrm{E} 2^{+/-}$and $\mathrm{E} 1^{-/-} \mathrm{E} 2^{+/+}$(hereafter pooled and dubbed 1-SKOs). $\mathrm{E} 1^{+/+} \mathrm{E} 2^{+/+} \mathrm{E} 3^{+/+}$with the same genetic background (approximately 80\% C57BL/6J $+20 \%$ SV129) were bred to generate wild-type controls (Wt) for electron microscopy, immunohistochemistry, and physiology experiments. For gene expression studies and a set of cell physiology experiments, we employed C57BL/6J mice as Wt controls. Most experiments were performed at 2-3 weeks of age (after hearing onset around postnatal day p12 in mice; Mikaelian \& Ruben, 1965), except for (i) cell physiology on TKO mice, which due to perinatal lethality were used within hours after birth to prepare organotypic cultures of organs of Corti, and (ii) auditory brainstem responses that were recorded at 6-8 weeks (taking into consideration that the C57BL/6J background is genetically predisposed for early onset age-related hearing loss; Shnerson \& Pujol, 1981). Both male and female mice were used for all experimental paradigms. 


\section{Single-cell RT-PCR}

To determine the expression of the three endophilin- $A$ genes in IHCs, we isolated mRNA from single IHCs of C57BL/6J mice at p14-16. In these experiments, individual IHCs were harvested from the apical coils of freshly dissected organs of Corti after cleaning off supporting cells. The filtered bath solution contained (in mM) $5.36 \mathrm{KCl}, 141 \mathrm{NaCl}, 0.5 \mathrm{MgSO}_{4} \cdot 7 \mathrm{H}_{2} \mathrm{O}, 10$ HEPES, $1 \mathrm{MgCl}_{2}, 1.3$ $\mathrm{CaCl}_{2}(\mathrm{pH} 7.2, \sim 300 \mathrm{mOsm} / \mathrm{l})$ and was continuously perfused at high rate $(1.7-3.3 \mathrm{ml} / \mathrm{min})$ to clear off cell debris. Individual IHCs were aspirated into a glass pipette containing $8 \mu \mathrm{l}$ of intracellular solution ( $135 \mathrm{mM} \mathrm{KCl,} 10 \mathrm{mM}$ HEPES, $0.5 \mathrm{mM} \mathrm{MgCl}_{2} \cdot 6 \mathrm{H}_{2} \mathrm{O}$ ). The pipette content was then transferred into first-strand cDNA synthesis mix containing after dilution (in $\mathrm{mM}$ ): 50 Tris- $\mathrm{HCl}$ ( $\mathrm{pH} 8.4$ ), 50 $\mathrm{KCl}, 5 \mathrm{MgCl}_{2}, 10 \mathrm{DTT}$, and 50 units of SuperScript II Reverse Transcriptase (Invitrogen, Carlsbad, CA) and 40 units of RNaseOUT ribonuclease inhibitor (Invitrogen). Aspirated bath solution in close proximity to the IHCs before and after IHC harvesting was used as a negative control. Reverse transcription (RT) was performed with SuperScript ${ }^{\mathrm{TM}}$ II RT and SuperScript ${ }^{\circledR}$ First-Strand Synthesis System for RT-PCR according to the manufacturer's instructions using oligo(dT) primers and random hexamers (Invitrogen). After ethanol precipitation, cDNA was pre-amplified with 20 cycles of a multiplex PCR, and the reaction product was used to perform quantitative real-time polymerase chain reaction (qPCR) using SYBR $^{\circledR}$ Green (Thermo Fisher). Sequences of endophilin-A-specific primers are listed in Appendix Table S1. Each sample was processed as technical duplicates using QuantStudio ${ }^{\mathrm{TM}} 6$ Flex (Life Technologies). Data were analyzed with QuantStudio ${ }^{\text {TM }}$ Real-Time PCR software.

\section{Immunoblotting of cochlear extracts}

Detection of endophilin-A1-3 protein from cochlear lysates was performed by Western blotting essentially as described in Vogl et al (2017). In brief, p15 animals of all respective genotypes were sacrificed and cochleae, hippocampi, and cerebellar fragments were dissected in ice-cold PBS. Additionally, modioli and organs of Corti were carefully micro-dissected and respectively pooled from $10 \mathrm{Wt}$ animals to assess expression in the two tissue fractions. Pooled cochleae of individual animals as well as the selected other tissue samples were transferred to fresh tubes containing a modified RIPA lysis buffer with the following composition (in mM): Tris- $\mathrm{HCl}(\mathrm{pH}$ 7.5) (50), $\mathrm{NaCl}$ (150), Na-deoxycholate (1), EDTA (1), PMSF (1), NaF (1), 1\% IGEPAL (v/v), 0.25\% (w/v), PhosSTOP phosphatase inhibitors, and complete protease inhibitors (Roche Holding AG, Basel, Switzerland). After manual homogenization of the tissue with a Teflon plunger and initial incubation for $30 \mathrm{~min}$ on ice, samples were transferred to a rotating wheel and gently lysed for an additional $2.5 \mathrm{~h}$ at $4^{\circ} \mathrm{C}$. Subsequently, samples were centrifuged at $1,000 \mathrm{~g}$ for $15 \mathrm{~min}$ at $4^{\circ} \mathrm{C}$ to precipitate large cell debris and bone fragments. Thereafter, the protein concentrations of the sample supernatants were determined using a BCA protein determination assay (Thermo Fisher) and the remaining samples were boiled for $6 \mathrm{~min}$ at $95^{\circ} \mathrm{C}$ in SDS sample buffer $(62.5 \mathrm{mM}$ Tris- $\mathrm{HCl}$ ( $\mathrm{pH} \mathrm{6.8),}$ $10 \%$ (v/v) glycerin, $2 \%$ SDS (w/v), $5 \%$ (v/v) $\beta$-mercaptoethanol, $0.002 \%(\mathrm{w} / \mathrm{v})$ brome phenol blue). Then, $10 \mu \mathrm{g}$ of protein per sample was subjected to $12 \%$ SDS-PAGE, transferred onto PVDF membranes, and-after blocking with $5 \%$ (w/v) milk powder dissolved in TBS-Tween-probed with the following primary antibodies: goat anti-endophilin-A1 (sc-10875; Santa Cruz), rabbit antiendophilin-A2 (a kind gift of Pietro de Camilli, Yale University, USA), goat anti-endophilin-A3 (sc-10880; Santa Cruz or Abcam, ab184008), and mouse anti- $\gamma$-adaptin (Cat. No.: 610386; BD Transduction Laboratories). After extensive washing, species-specific peroxidase-conjugated secondary antibodies were applied for chemiluminescent detection on an Intas ChemoCam imaging platform. In our experiments, none of the tested A3 antibodies showed selectivity for A3 but rather appeared to (also) detect A1.

\section{Immunohistochemistry and confocal microscopy of immunolabeled hair cells}

Freshly dissected apical cochlear turns of 2-week-old mice were fixed with (i) $4 \%$ formaldehyde (FA) in phosphate-buffered saline (PBS) - depending on the experimental paradigm either for 20 or for $60 \mathrm{~min}$-on ice, or (ii) for $20 \mathrm{~min}$ in methanol at $-20^{\circ} \mathrm{C}$ ( $\mathrm{Ca}_{\mathrm{V}} 1.3$-staining). Thereafter, specimens were washed in PBS and incubated for $1 \mathrm{~h}$ in goat serum dilution buffer (GSDB: 16\% normal goat serum, $450 \mathrm{mM} \mathrm{NaCl}, 0.3 \%$ Triton X-100, $20 \mathrm{mM}$ phosphate buffer, $\mathrm{pH}$ 7.4) in a wet chamber at room temperature. Primary antibodies were dissolved in GSDB and applied for $3 \mathrm{~h}$ at room temperature $\left(\mathrm{Ca}_{V} 1.3\right.$-staining), or overnight at $4^{\circ} \mathrm{C}$ in a wet chamber. After washing $3 \times 10 \mathrm{~min}$ (wash buffer: $450 \mathrm{mM} \mathrm{NaCl}, 20 \mathrm{mM}$ phosphate buffer, $0.3 \%$ Triton X-100), the tissue was incubated with GSDB-diluted secondary antibodies in a light-protected wet chamber for $1 \mathrm{~h}$ at room temperature. Then, the specimens were washed in wash buffer and finally in $5 \mathrm{mM}$ phosphate buffer and mounted onto glass microscope slides with mounting medium (Mowiol). The following primary antibodies were used: mouse monoclonal anti-CtBP2 (also recognizing the ribbon protein RIBEYE, 1:200, BD Biosciences, Cat. No. 612044), mouse antiotoferlin (1:300, Abcam, Cat. No. ab53233), rabbit anti-vGlut3 (1:300, Synaptic Systems, Cat. No. 135 203), rabbit anti-Ca 1.3 (1:30, Alomone Labs, Cat. No. ACC 005), and rabbit polyclonal myosin 6 (1:300, Proteus Biosciences Inc., \#25-6791). The secondary antibodies used for (i) confocal microscopy were goat anti-rabbit Alexa Fluor 488 and goat anti-mouse Alexa Fluor 568 (1:200, Invitrogen, Cat. No. A 11008 and A 11004, respectively), and (ii) STED were STAR 580-tagged goat anti-mouse (1:200, Abberior, Cat. No. 2-0002-005-1) and STAR 635P-tagged goat anti-rabbit (1:200, Abberior, Cat. No. 2-0012-007-2). Confocal images were acquired using a laser scanning confocal microscope (Leica TCS SP5, Leica Microsystems GmbH, Mannheim, Germany, and Zeiss LSM800, Carl Zeiss AG, Oberkochen, Germany) with $488 \mathrm{~nm}$ (Ar) and $561 \mathrm{~nm}$ (He-Ne) lasers for excitation and $1.4 \mathrm{NA} 63 \times$ oil immersion objectives. Z-axis stacks from comparable tonotopic regions were acquired with a pixel size of $80 \times 80 \mathrm{~nm}$ and step size of (i) $0.6 \mu \mathrm{m}$ for otoferlin quantifications and (ii) $0.25 \mu \mathrm{m}$ for ribbon counting. 2D STED images were acquired with a pixel size of $15 \times 15 \mathrm{~nm}$ using an Abberior Instruments Expert Line $775 \mathrm{~nm}$ 2-color STED microscope (Abberior Instruments, Göttingen, Germany), with excitation lasers at 561 and $640 \mathrm{~nm}$ and a STED laser at $775 \mathrm{~nm}, 1.2 \mathrm{~W}$, using a $1.4 \mathrm{NA} 100 \times$ oil immersion objective. Images were processed using ImageJ (http://imagej.net/) and assembled for display in Adobe Illustrator software. 


\section{Patch clamp of IHCs from cultured neonatal organs of Corti}

Organotypic cultures of perinatal (p0) mice were prepared as described previously (Nouvian et al, 2011; Reisinger et al, 2011; Vogl et al, 2015). Briefly, organs of Corti were dissected from TKO, 1/3-DKO, or C57BL/6J mice in HEPES-HBSS supplemented with $250 \mathrm{ng} / \mathrm{ml}$ fungizone (Life Technologies) and $10 \mu \mathrm{g} / \mathrm{ml}$ Penicillin G (Sigma-Aldrich), mounted on Cell-Tak ${ }^{\mathrm{TM}}$-coated coverslips (BD Biosciences) and incubated in DMEM/F12 with 5\% FBS. During the culture period of 1 week, half of the medium was replaced with fresh culture medium every second day. After 7 days in culture, perforated patch-clamp recordings were performed on apical coil IHCs using an extracellular solution containing (in mM) $103 \mathrm{NaCl}$, $2.8 \mathrm{KCl}, 1 \mathrm{MgCl}_{2}, 10$ HEPES, 35 TEA-Cl, $11.2 \mathrm{D}$-glucose, $10 \mathrm{CaCl}_{2}$, and apamin $(100 \mathrm{nM})$ to inhibit small conductance (SK) $\mathrm{K}^{+}$channels ( $\mathrm{pH} 7.2, \sim 300 \mathrm{mOsm} / \mathrm{l}$ ), and an intracellular solution containing (in mM) 129 Cs-gluconate, 10 TEA-Cl, 10 4-AP, 10 HEPES, 1 $\mathrm{MgCl}_{2}$, and amphotericin B $(300 \mu \mathrm{g} / \mathrm{ml})(\mathrm{pH} 7.2, \sim 280 \mathrm{mOsm} / \mathrm{l})$. In a subset of experiments, the extracellular $\mathrm{Ca}^{2+}$ concentration $\left(\left[\mathrm{Ca}^{2+}\right]_{\mathrm{e}}\right)$ was lowered to $6 \mathrm{mM}$ to reduce presynaptic $\mathrm{Ca}^{2+}$ influx in C57BL/6J mice; this was balanced by a complementary increase in $\mathrm{NaCl}$ to maintain overall ionic strength. All experiments were done at room temperature $\left(22-24^{\circ} \mathrm{C}\right)$ using an EPC10 amplifier with PatchMaster software (HEKA, Elektronik, Lambrecht, Germany). $\mathrm{Ca}^{2+}$ current and membrane capacitance measurements were performed using the Lindau-Neher technique (Lindau \& Neher, 1988; Moser \& Beutner, 2000). Currents were leak-subtracted with a $\mathrm{p} / 10$ protocol. Liquid junction potentials $(17 \mathrm{mV})$ were corrected offline.

\section{Patch clamp of IHCs in acutely dissected organs of Corti}

Patch-clamp recordings from IHCs of freshly dissected organs of Corti (apical coil) from p14-18 mice were essentially performed as described previously (Moser \& Beutner, 2000). For recordings of $\mathrm{Ca}^{2+}$ current, exocytosis, and endocytosis, the pipette solution contained (in mM) 129 Cs-gluconate, 10 TEA-Cl, 10 4-AP, 10 HEPES, $1 \mathrm{MgCl}_{2}$, and amphotericin B $(300 \mu \mathrm{g} / \mathrm{ml})$ (pH 7.2, $\sim 280 \mathrm{mOsm} / \mathrm{l}$ ). The extracellular solution contained (in $\mathrm{mM}$ ) 105 $\mathrm{NaCl}, 35 \mathrm{TEA}-\mathrm{Cl}, 2.8 \mathrm{KCl}, 2 \mathrm{CaCl}_{2}, 1 \mathrm{MgCl}_{2}, 5$ 4-AP, $1 \mathrm{CsCl}, 10$ HEPES, and 11.1 D-glucose (pH 7.2, 300 mOsm/l). EPC-9 amplifiers (HEKA) controlled by Pulse software (HEKA) were used for measurements. All voltages were corrected offline for the liquid junction potential $(14 \mathrm{mV})$. Currents were leak-corrected using a p/ 10 protocol for exocytosis or a p/20 protocol for endocytosis. Cells with leak currents exceeding $-35 \mathrm{pA}$ at $-84 \mathrm{mV}$ holding potential or with a series resistance higher than $30 \mathrm{M} \Omega$ were excluded from the analysis. Current-voltage (IV) relationships were obtained by applying 10-ms pulses of increasing voltage. For capacitance $\left(\mathrm{C}_{\mathrm{m}}\right)$ measurements, IHCs were stimulated by depolarizations of different durations to $-14 \mathrm{mV}$ at intervals of $20-120 \mathrm{~s}$.

\section{Electron microscopy and tomography}

Conventionally embedded samples for electron microscopy and tomography were prepared as described previously (Jung et al, 2015). Apical turns of the organ of Corti were acutely dissected and either stimulated for $15 \mathrm{~min}$ at room temperature with a solution containing (in mM) $50 \mathrm{KCl}, 95 \mathrm{NaCl}, 1 \mathrm{MgCl}_{2}, 5 \mathrm{CaCl}_{2}, 10$ HEPES and subsequently fixed for $1 \mathrm{~h}$ on ice with $4 \%$ PFA and $0.5 \%$ glutaraldehyde in PBS, or immediately fixed for $1 \mathrm{~h}$ on ice with the same fixative. Subsequently, samples were post-fixed overnight on ice with secondary fixative comprising $2 \%$ glutaraldehyde in $0.1 \mathrm{M}$ sodium cacodylate buffer, $\mathrm{pH}$ 7.2. The next day, samples were washed thrice in sodium cacodylate buffer, before $1 \%$ osmium tetroxide ( $(\mathrm{v} / \mathrm{v})$ in $0.1 \mathrm{M}$ sodium cacodylate buffer) was applied for post-fixation for $1 \mathrm{~h}$ on ice. After $2 \times 10 \mathrm{~min}$ washing steps in sodium cacodylate buffer and three brief washing steps in distilled water, the samples were stained en bloc with $1 \%$ uranyl acetate $((\mathrm{v} / \mathrm{v})$ in distilled water) for $1 \mathrm{~h}$ on ice and briefly washed with distilled water. The dehydration was performed on ice in solutions with increasing ethanol concentrations, and the samples were subsequently infiltrated in Epon resin (100\% EtOH/Epon 1:1 (v/v), 30 and $90 \mathrm{~min} ; 100 \%$ Epon, overnight), placed into embedding molds, and polymerized for $48 \mathrm{~h}$ at $70^{\circ} \mathrm{C}$. $65-\mathrm{nm}$ sections of the embedded samples were obtained approaching from the anterior edge using an ultramicrotome (UC6, Leica Microsystems) and placed on formvar-coated $2 \times 1 \mathrm{~mm}$ copper slot grids. Sections were post-fixed and post-stained with uranyl acetate or uranyl acetate replacement (Science Services, Munich) and lead citrate following standard protocols. Micrographs were acquired with a JEM 1011 electron microscope (JEOL) equipped with a Gatan Orius 1200A camera using the Digital Micrograph software package at a 5,000fold to 12,000-fold magnification.

Electron tomography was essentially performed as described previously (Jung et al, 2015; Strenzke et al, 2016). Briefly, 250-nm sections were applied to formvar-coated copper mesh grids and post-stained as described above. $10 \mathrm{~nm}$ gold beads were applied to both sides of the grid as fiducial markers. Tilt series were acquired with a JEOL electron microscope (JEM 2100) from $-60^{\circ}$ to $+60^{\circ}$ with an increment of $1^{\circ}$ using Serial-EM software. Tomograms were generated using the IMOD package etomo, and models were generated using $3 \mathrm{dmod}$ (bio3d.colorado.edu/imod/).

\section{Auditory brainstem responses}

Recordings of ABRs (auditory brainstem responses) and DPOAE (distortion product otoacoustic emissions) were performed as previously described (Jing et al, 2013). In brief, mice were anesthetized with a combination of ketamine $(125 \mathrm{mg} / \mathrm{kg})$ and xylazine $(2.5 \mathrm{mg} /$ kg) i.p. The core temperature was maintained constant at $37^{\circ} \mathrm{C}$ using a heat blanket (Hugo Sachs Elektronik-Harvard Apparatus). For stimulus generation, presentation, and data acquisition, we used the TDT II system run by BioSig software (Tucker Davis Technologies) (MathWorks). Tone bursts $(4 / 6 / 8 / 12 / 16 / 24 / 32 \mathrm{kHz}, 10 \mathrm{~ms}$ plateau, $1 \mathrm{~ms} \cos ^{2}$ rise/fall) or clicks of $0.03 \mathrm{~ms}$ were presented at $40 \mathrm{~Hz}$ (tone bursts) or $20 \mathrm{~Hz}$ (clicks) in the free field ipsilaterally using a JBL 2402 speaker. The difference potential between vertex and mastoid subdermal needles was amplified 50,000 times, filtered $(400-4,000 \mathrm{~Hz})$, and sampled at a rate of $50 \mathrm{kHz}$ for $20 \mathrm{~ms}, 1,300$ times, to obtain two mean ABR traces for each sound intensity.

\section{Purification of recombinant endophilin-A1}

Plasmid pGEX6P-endopilin-A1 (rat) was a kind gift of Prof. P. De Camilli (Yale University, New Haven CT, USA). After an overnight 
expression $\left(16^{\circ} \mathrm{C}\right.$ for $\sim 9 \mathrm{~h}$ ), in E. coli BL21(DE3) (Sigma) the protein was purified on a GST-glutathione affinity column (GE Healthcare, Piscataway, NJ). The GST-tag was cleaved using PreScission protease (GE Healthcare, Piscataway, NJ), and the sample was then subjected to gel filtration chromatography (Superdex 200 10/300 GL; Amersham Pharmacia Biosciences, Piscataway, NJ) in $20 \mathrm{mM}$ Tris (pH 7.4), $300 \mathrm{mM} \mathrm{KCl}, 5 \mathrm{mM}$ imidazole, $1 \mathrm{mM}$ DTT. The protein concentration was determined $(14.5 \mathrm{mg} / \mathrm{ml})$, and aliquots of the purified protein were stored at $-80^{\circ} \mathrm{C}$ until being used as detailed below.

\section{Immunoprecipitation experiments}

The immunoprecipitation experiments were performed as described in Murdoch et al (2016). Briefly, HeLa cells (ATCC ${ }^{\circledR}$ CCL-2; $1.5 \times 10^{6}$ ) were plated in a $\varnothing 10-\mathrm{cm}$ plate and transfected with pmRFP-endophilin-A1 (a gift of P. De Camilli, Yale University, New Haven, CT, USA) and pEGFP-otoferlin (gift of T. Weber, University Medical Center Göttingen), or pEGFP-N1 (Clontech) for control, using Fugene (Invitrogen) $3 \mathrm{~h}$ after plating (the total DNA amount was $8 \mu \mathrm{g} /$ plate). On average, $75-80 \%$ of HeLa cells were transfected. Cells were harvested $72 \mathrm{~h}$ after transfection and lysed in $10 \mathrm{mM}$ Tris/Cl (pH 7.5), $150 \mathrm{mM} \mathrm{NaCl}, 0.5 \mathrm{mM}$ EDTA, 0.5\% NP40. The lysates were then incubated for $2 \mathrm{~h}$ at $4^{\circ} \mathrm{C}$ with ChromoTek Trap ${ }^{\circledR}$-GFP agarose beads (ChromoTek, Germany) following manufacturer's protocol (GFP-Trap ${ }^{\circledR}$ A A for immunoprecipitation of GFPfusion proteins from mammalian cell extract). After binding, protein GST-Trap_A beads were resuspended in ice-cold dilution buffer and centrifuged. The washing step was repeated twice according to the standard protocol. Fractions (input $=$ cell lysate; bound $=$ beads with bound proteins, non-bound = supernatant) were loaded onto SDS-PAGE gel followed by an immunoblot analysis against endophilin-A1 (Endo A1 antibody, L-18, Santa Cruz, USA), RFP (5F8; ChromoTek, Germany), using standard procedure and Odyssey ${ }^{\circledR}$ imaging system (LI-COR, Lincoln, NE, USA). Three independent experiments were performed.

To check whether otoferlin binds directly to endophilin, HeLa cells $\left(\right.$ ATCC $^{\circledR}$ CCL-2; $1.2 \times 10^{6}$ ) were plated in a $\varnothing 10-\mathrm{cm}$ plate and transfected with pEGFP or pEGFP-otoferlin $(7 \mu \mathrm{g} /$ plate in total) using Fugene (Invitrogen). 75-80\% of HeLa cells were transfected on average. Cells were harvested $72 \mathrm{~h}$ after transfection and lysed in $10 \mathrm{mM}$ Tris/Cl (pH 7.5), $150 \mathrm{mM} \mathrm{NaCl}, 0.5$ mM EDTA, 0.5\% NP-40. EGFP or otoferlin-EGFP was then bound to Trap ${ }^{\circledR}$-GFP agarose beads (ChromoTek) following manufacturer's instructions, and the beads were incubated with $50 \mu \mathrm{g}$ of purified endophilin-A1 protein for $2 \mathrm{~h}$ at $4^{\circ} \mathrm{C}$. After centrifugation $\left(2,500 \mathrm{~g}, 2 \mathrm{~min}, 4^{\circ} \mathrm{C}\right)$, two fractions were obtained: beads with bound proteins (bound) and supernatant (nonbound). In addition to cell lysates (input), these fractions were loaded onto SDS-PAGE gel followed by an immunoblot analysis against endophilin-A1 (EndoA1 antibody L-18, Santa Cruz, USA) using standard procedure and Odyssey ${ }^{\circledR}$ imaging system (LI-COR, Lincoln, NE, USA). Two independent experiments were made.

\section{Data analysis}

\section{Immunohistochemistry}

IHC synaptic ribbons were manually counted in confocal $z$-projections of immunolabeled organs of Corti using ImageJ software.
Analysis of $\mathrm{Ca}_{V} 1.3$ STED imaging data was performed in Igor Pro 6.3 software (Wavemetrics). The area of spot- and line-shaped $\mathrm{Ca}^{2+}$ channel clusters was estimated by fitting a 2D Gaussian function (genetic fit algorithm; Sanchez del Rio \& Pareschi, 2001) to the individual clusters in 2D STED images to obtain the full width at half maximum (FWHM) of the long and short axes. Subsequently, areas of the clusters were calculated by the following formula: area $=\pi \times($ long axis $/ 2) \times($ short axis $/ 2)$. Otoferlin levels of IHCs were semi-quantitatively assessed as immunofluorescence intensity values and analyzed using Imaris (Bitplane) and MATLAB (MathWorks) as described before (Strenzke et al, 2016). Otoferlin intensity line scans along the longitudinal axis of IHCs were generated from single sections using ImageJ; Igor Pro 6.3 software was used to generate average intensity profiles after normalizing the intensity profiles of the individual cells to their lengths.

\section{Patch-clamp electrophysiology}

Electrophysiological data were analyzed using custom-written programs in Igor Pro 6.3. For analysis of IV curves, the last $5 \mathrm{~ms}$ of the evoked $\mathrm{Ca}^{2+}$ current was averaged. For measurements of exocytosis, total $\mathrm{Ca}^{2+}$ charge $\left(\mathrm{Q}_{\mathrm{Ca}}\right)$ was estimated by taking the integral of the leak-subtracted current during the depolarization step and the exocytic increment in capacitance $\left(\Delta \mathrm{C}_{\mathrm{m}}\right)$ was quantified as the difference in the averaged $C_{m} 400 \mathrm{~ms}$ before and after (skipping the first 60 or $100 \mathrm{~ms}$ ) the depolarization. Mean estimates of individual IHCs were calculated from two to four rounds of exocytosis, and were used to calculate grand averages of IHCs per genotype. This avoided dominance of IHCs contributing more sweeps. Only IHCs with reproducible $\Delta \mathrm{C}_{\mathrm{m}}$ among the individual rounds were included.

Endocytosis was assessed as described previously (Neef et al, 2014). IHCs showed a slow depolarization-independent decrease in $\mathrm{C}_{\mathrm{m}}$ during the perforated patch-clamp recording. To correct for this, we used two independent methods: (i) by fitting and subtracting an exponential function to the baseline $\mathrm{C}_{\mathrm{m}}$ as recorded during the entire experiment (Fig EV4A, $\mathrm{C}$ and $\mathrm{C}^{\prime}$ ) or (ii) by fitting and subtracting a linear function to the last $10 \mathrm{~s}$ of a non-depolarizing pulse recorded before the actual trace (Fig EV4D and $\mathrm{D}^{\prime}$ ). The results obtained using both methods were comparable (Fig EV4C" and $\mathrm{D}^{\prime \prime}$ ). We favor the first method, which seems to perform a better correction, evident from the least divergence of the recorded traces for each individual IHC (for an example, see Fig EV4C).

The endocytic decline in $\mathrm{C}_{\mathrm{m}}$ following a depolarization-induced exocytic $\Delta \mathrm{C}_{\mathrm{m}}$ was measured over $20 \mathrm{~s}$ following a 2-s-long recording of $C_{m}$ baseline and 20 or $200 \mathrm{~ms}$ of depolarization. After correction, we determined the residual slope of the $2 \mathrm{~s}$ preceding the depolarization (residual pre-slope) and excluded those IHCs where the residual pre-slope exceeded the average \pm 2 times the standard deviation for each genotype (for $20 \mathrm{~ms}$, one cell was excluded for each genotype; for $200 \mathrm{~ms}$, one cell was excluded for the Wt and for the $1 / 3$-DKO). Even though the residual pre-slope exhibits a negative trend (for $20 \mathrm{~ms}$ : $-0.51 \pm 0.24 \mathrm{fF} / \mathrm{s}$ for $\mathrm{Wt},-0.20 \pm 0.28 \mathrm{fF} / \mathrm{s}$ for $1 / 2$-DKO, and $-0.62 \pm 0.22 \mathrm{fF} / \mathrm{s}$ for $1 / 3$-DKO; for $200 \mathrm{~ms}$ : $-0.27 \pm 0.37 \mathrm{fF} / \mathrm{s}$ for $\mathrm{Wt},-0.28 \pm 0.23 \mathrm{fF} / \mathrm{s}$ for $1 / 2-\mathrm{DKO}$, and $0.036 \pm 0.22 \mathrm{fF} / \mathrm{s}$ for $1 / 3-\mathrm{DKO}$ ), it is not statistically different between the genotypes (for $20 \mathrm{~ms}$ : one-way ANOVA, $F(2,35)=$ $0.80, P=0.4507$; for $200 \mathrm{~ms}$ : one-way ANOVA, $F(2,36)=0.45$, $P=0.6391)$. 
Analysis of the linear component of endocytosis following brief depolarizations (20 ms) was performed by fitting a linear function to the post-depolarization $\mathrm{C}_{\mathrm{m}}$ data, skipping the first $200 \mathrm{~ms}$, and noting the point at which the line fit or its extrapolation in time returned to baseline $C_{m}$. Responses in which $C_{m}$ did not return to baseline within $80 \mathrm{~s}$ were excluded from this analysis. The number of IHCs where $\mathrm{C}_{\mathrm{m}}$ did not return to baseline was low for all genotypes: Wt (3 out of 17 recorded IHCs), 1/2-DKO ( 0 cells out of 14), and $1 / 3$-DKO ( 3 cells out of 17 ), but many IHCs regardless of genotype showed at least one trace without $\mathrm{C}_{\mathrm{m}}$ return within $80 \mathrm{~s}$.

We analyzed the exponential component of $\mathrm{C}_{\mathrm{m}}$ decline following 200-ms-long depolarization by fitting a linear function to the final $10 \mathrm{~s}$ of $\mathrm{C}_{\mathrm{m}}$ data and subtracting the extrapolated fit. The residual was then fitted with an exponential function using a genetic curve fitting algorithm (Sanchez del Rio \& Pareschi, 2001). In few cases, the fitting window for the linear component was slightly adjusted to exclude $\mathrm{C}_{\mathrm{m}}$ artifacts. For some cells, the $\mathrm{C}_{\mathrm{m}}$ decline did not exhibit an exponential component ( 1 out of 12 IHCs for Wt, 3 out of 13 for $1 / 2$-DKO, and 3 out of 14 for $1 / 3-\mathrm{DKO})$. Therefore, they were excluded from the statistical analysis of the amplitude or time constant of the exponential component; yet, they are still included for the slope of the linear component for $200 \mathrm{~ms}$.

\section{Auditory brainstem recordings}

Recordings were performed as described (Jing et al, 2013). In brief, hearing threshold was determined with $10 \mathrm{~dB}$ precision as the lowest stimulus intensity that evoked a reproducible response waveform in both traces by visual inspection.

\section{Transmission electron microscopy (TEM)}

Quantification of electron microscopy data was performed with ImageJ for random sections, and with IMOD software for tomograms. In random sections, the ribbon proximity (Fig 7) was defined as area around the ribbon in $1 \mu \mathrm{m}$ distance from the center of the synaptic ribbon. For each section, the exact area of the intracellular cytoplasm was measured, whereby the area of mitochondria was excluded. Numbers of small, clear vesicles (max. diameter $70 \mathrm{~nm}$ between outer leaflets of the vesicle membrane; Figs 7B and 9D) except of the vesicles in the first row around the ribbon-were counted and divided by the respective area. For quantification of endosome-like vacuoles (ELVs), the area of all ELVs per section was cumulated and divided by the cytoplasmic area (Figs 7C and 9E). For coated structures, the following subgroups were defined: (i) coated vesicles with a maximum outer diameter of $70 \mathrm{~nm}$; (ii) coated vacuoles with an outer diameter of more than $70 \mathrm{~nm}$ as well as tubular structures fully covered by a coat; (iii) coated pits containing a connection to the plasma membrane; and (iv) coated pits on otherwise uncoated ELVs. For quantification of SVs around the ribbon, the following analysis criteria were used for random sections: Ribbon height was measured from the top of the ribbon to the bottom excluding the presynaptic density; the distal half of the ribbon was defined as the upper $50 \%$ of the total ribbon length. SVs were allocated to the distal or proximal halves of the ribbon if more than $50 \%$ of the SV area were located in the respective region (Fig $8 \mathrm{C}^{\prime}$ ). Moreover, two distinct SV pools were defined: (i) The ribbon-associated pool contained all SVs in the first row around the ribbon with a maximum distance of $80 \mathrm{~nm}$ (RA-SVs, Figs $8 \mathrm{C}$ and 9B) and (ii) the membrane-proximal SV pool (MP-SVs), which was formed by SVs in the first layer above the AZ membrane within a maximum distance of $<25 \mathrm{~nm}$ to the plasma membrane and a maximum lateral distance of $<100 \mathrm{~nm}$ to the presynaptic density (Figs 8D and 9C).

\section{Electron tomography}

For quantification of tomograms, the definition of MP-SVs was adjusted to include all SVs with a maximal distance of $50 \mathrm{~nm}$ to the AZ membrane that were no more than $100 \mathrm{~nm}$ apart from the presynaptic density (all criteria being valid at the maximum projection of the respective SV in the tomogram, no changes to RA-SV definition). This adjustment was made not only to guarantee for comparability with other recent ultrastructural studies of SV pools at ribbon synapses, but also because the high z-resolution of virtual sections from tomograms facilitates the identification of tethers (also including lengths $>25 \mathrm{~nm}$ ) linking MP-SVs to the presynaptic density and to the AZ membrane. Since $65-\mathrm{nm}$ random sections do not provide visualization of tethers, we chose a stricter criterion of $25 \mathrm{~nm}$ maximal distance to avoid over-interpretation of the MP-SV pool. Nevertheless, we re-quantified all tomography data also with the criteria used for random sections resulting in overall unchanged significance values. For quantification of ELVs, the minimal distance between ELV and ribbon was measured; only ELVs within a distance of $200 \mathrm{~nm}$ were included. All distances for random sections and for tomograms were measured between the outer membrane of SVs and the respective structure.

\section{Experimental design and statistical analysis}

Sample sizes were chosen according to typical observation numbers used on each respective field (e.g., immunohistochemistry, cellular or systems electrophysiology, electron microscopy), and can be found both in the respective figures and in the corresponding figure legends. Data were analyzed using MATLAB (MathWorks), Excel (Microsoft), Igor Pro 6 (Wavemetrics), Origin 9.0 (Microcal software), and GraphPad Prism (GraphPad software). Averages are expressed as mean $\pm \mathrm{SEM}$ or mean $\pm \mathrm{SD}$, as specified. Data sets were tested for normal distribution (Jarque-Bera test, D'Agostino and Pearson omnibus normality test, or the Shapiro-Wilk test) and equality of variances. Statistical significance was calculated using one-way ANOVA test followed by Tukey's test for normally distributed data, or Kruskal-Wallis $(\mathrm{K}-\mathrm{W})$ test followed by post hoc Dunn's test for non-normally distributed data. Significant differences are reported as $* P<0.05,{ }^{*} P P<0.01$, and $* * * P<0.001$. Box plots are drawn from 25 to $75 \%$, whiskers indicate $10-90 \%$ of data points, and squares show the averages. For the endocytosis data (Fig 6), the ROUT method from GraphPad Prism was used to identify outliers $(\mathrm{Q}=1 \%)$.

Expanded View for this article is available online.

\section{Acknowledgements}

We thank N. Dietrich, T. Goldak, S. Gerke, C. Senger-Freitag, and M. Costa for expert technical assistance. This work was supported by grants of the German Research Foundation through the collaborative research center 889 (project A8 to I.M. and T.M. and A7 to C.W.), the Emmy-Noether program (to I.M.), and the Leibniz program (to T.M.). C.V. is a Creutzfeldt Fellow of the Elisabeth and Helmut Uhl Foundation. L.M.J.T received an Erasmus Mundus scholarshipNeurasmus. I.K. receives a fellowship from the Engelhorn Stiftung. 


\section{Author contributions}

IM, CW, and TM designed the study. JK performed electron microscopy, electron tomography, and immunohistochemistry followed by confocal and STED microscopy, and handled mouse breeding. LMJT performed patch-clamp capacitance measurements and immunohistochemistry, and contributed to single-cell real-time PCR. CV performed organ of Corti cultures, patch-clamp capacitance measurements, immunohistochemistry and confocal microscopy, as well as Western blots, and contributed to single-cell real-time PCR. JN performed patch-clamp capacitance measurements and contributed to singlecell real-time PCR. IM and MK established and maintained endophilin colonies and performed single-cell real-time PCR. IM and IK performed biochemical interaction studies. CW supervised electron microscopy and tomography. NS supervised auditory systems physiology. All authors performed data analysis. TM, IM, JK, LMJT, and CV prepared the manuscript with contributions of all other authors.

\section{Conflict of interest}

The authors declare that they have no conflict of interest.

\section{References}

Anggono V, Robinson PJ (2007) Syndapin I and endophilin I bind overlapping proline-rich regions of dynamin I: role in synaptic vesicle endocytosis. J Neurochem 102: $931-943$

Bai J, Hu Z, Dittman JS, Pym ECG, Kaplan JM (2010) Endophilin functions as a membrane-bending molecule and is delivered to endocytic zones by exocytosis. Cell 143: $430-441$

Beutner D, Voets T, Neher E, Moser T (2001) Calcium dependence of exocytosis and endocytosis at the cochlear inner hair cell afferent synapse. Neuron 29: 681-690

Boumil RM, Letts VA, Roberts MC, Lenz C, Mahaffey CL, Zhang Z, Moser T, Frankel WN (2010) A missense mutation in a highly conserved alternate exon of dynamin-1 causes epilepsy in fitful mice. PLoS Genet 6: e1001046

Brandt A, Striessnig J, Moser T (2003) CaV1.3 channels are essential for development and presynaptic activity of cochlear inner hair cells. J Neurosci 23: 10832-10840

Brandt A, Khimich D, Moser T (2005) Few CaV1.3 channels regulate the exocytosis of a synaptic vesicle at the hair cell ribbon synapse. J Neurosci 25: $11577-11585$

Chen Y, Deng L, Maeno-Hikichi Y, Lai M, Chang S, Chen G, Zhang J (2003) Formation of an endophilin- $\mathrm{Ca}^{2+}$ channel complex is critical for clathrinmediated synaptic vesicle endocytosis. Cell 115: 37-48

Duncker SV, Franz C, Kuhn S, Schulte U, Campanelli D, Brandt N, Hirt B, Fakler B, Blin N, Ruth P, Engel J, Marcotti W, Zimmermann U, Knipper M (2013) Otoferlin couples to clathrin-mediated endocytosis in mature cochlear inner hair cells. J Neurosci 33: 9508-9519

Ferguson S, Raimondi A, Paradise S, Shen H, Mesaki K, Ferguson A, Destaing O, Ko G, Takasaki J, Cremona O, O’Toole E, De Camilli P (2009) Coordinated actions of actin and BAR proteins upstream of dynamin at endocytic clathrin-coated pits. Deu Cell 17: 811-822

Ferguson SM, De Camilli P (2012) Dynamin, a membrane-remodelling GTPase. Nat Reu Mol Cell Biol 13: 75-88

Frank T, Khimich D, Neef A, Moser T (2009) Mechanisms contributing to synaptic $\mathrm{Ca}^{2+}$ signals and their heterogeneity in hair cells. Proc Natl Acad Sci USA 106: $4483-4488$

Frank T, Rutherford MA, Strenzke N, Neef A, Pangršič T, Khimich D, Fejtova A, Fetjova A, Gundelfinger ED, Liberman MC, Harke B, Bryan KE, Lee A, Egner
A, Riedel D, Moser T (2010) Bassoon and the synaptic ribbon organize $\mathrm{Ca}^{2+}$ channels and vesicles to add release sites and promote refilling. Neuron 68: $724-738$

Gad H, Ringstad N, Löw P, Kjaerulff O, Gustafsson J, Wenk M, Di Paolo G, Nemoto Y, Crum J, Ellisman MH, De Camilli P, Shupliakov O, Brodin L (2000) Fission and uncoating of synaptic clathrin-coated vesicles are perturbed by disruption of interactions with the SH3 domain of endophilin. Neuron 27: 301-312

Guillet M, Sendin G, Bourien J, Puel J-L, Nouvian R (2016) Actin filaments regulate exocytosis at the hair cell ribbon synapse. J Neurosci 36: 649-654

Haucke V, Neher E, Sigrist SJ (2011) Protein scaffolds in the coupling of synaptic exocytosis and endocytosis. Nat Reu Neurosci 12: 127-138

jing Z, Rutherford MA, Takago H, Frank T, Fejtova A, Khimich D, Moser T, Strenzke N (2013) Disruption of the presynaptic cytomatrix protein bassoon degrades ribbon anchorage, multiquantal release, and sound encoding at the hair cell afferent synapse. J Neurosci 33: 4456-4467

Jung S, Maritzen T, Wichmann C, Jing Z, Neef A, Revelo NH, Al-Moyed H, Meese S, Wojcik SM, Panou I, Bulut H, Schu P, Ficner R, Reisinger E, Rizzoli So, Neef J, Strenzke N, Haucke V, Moser T (2015) Disruption of adaptor protein $2 \mu$ (AP- $2 \mu)$ in cochlear hair cells impairs vesicle reloading of synaptic release sites and hearing. EMBO J 34: 2686-2702

Kamin D, Revelo NH, Rizzoli SO (2014) FM dye photo-oxidation as a tool for monitoring membrane recycling in inner hair cells. PLOS ONE 9: e88353

Khimich D, Nouvian R, Pujol R, tom Dieck S, Egner A, Gundelfinger ED, Moser $T$ (2005) Hair cell synaptic ribbons are essential for synchronous auditory signalling. Nature 434: 889-894

Kokotos AC, Cousin MA (2015) Synaptic vesicle generation from central nerve terminal endosomes. Traffic 16: 229-240

Kononenko NL, Puchkov D, Classen GA, Walter AM, Pechstein A, Sawade L, Kaempf N, Trimbuch T, Lorenz D, Rosenmund C, Maritzen T, Haucke V (2014) Clathrin/AP-2 mediate synaptic vesicle reformation from endosome-like vacuoles but are not essential for membrane retrieval at central synapses. Neuron 82: 981-988

Kononenko NL, Haucke V (2015) Molecular mechanisms of presynaptic membrane retrieval and synaptic vesicle reformation. Neuron 85: 484-496

Lenzi D, Crum J, Ellisman MH, Roberts WM (2002) Depolarization redistributes synaptic membrane and creates a gradient of vesicles on the synaptic body at a ribbon synapse. Neuron 36: 649-659

Lindau M, Neher E (1988) Patch-clamp techniques for time-resolved capacitance measurements in single cells. Pflügers Archiv 411: 137-146

Melcher JR, Guinan JH Jr, Knudson IM, Kiang NYS (1996) Generators of the brainstem auditory evoked potential in cat II. Correlating lesion sites with waveform changes. Hear Res 93: 28-51

Meyer AC, Frank T, Khimich D, Hoch G, Riedel D, Chapochnikov NM, Yarin YM, Harke B, Hell SW, Egner A, Moser T (2009) Tuning of synapse number, structure and function in the cochlea. Nat Neurosci 12: 444-453

Mikaelian D, Ruben RJ (1965) Development of hearing in the normal Cba-J mouse: correlation of physiological observations with behavioral responses and with cochlear anatomy. Acta Otolaryngol 59: $451-461$

Milosevic I, Giovedi S, Lou X, Raimondi A, Collesi C, Shen H, Paradise S, O'Toole E, Ferguson S, Cremona O, De Camilli P (2011) Recruitment of endophilin to clathrin-coated pit necks is required for efficient vesicle uncoating after fission. Neuron 72: 587-601

Milosevic I (2018) Revisiting the role of clathrin-mediated endoytosis in synaptic vesicle recycling. Front Cell Neurosci 12: 27

Moser T, Beutner D (2000) Kinetics of exocytosis and endocytosis at the cochlear inner hair cell afferent synapse of the mouse. Proc Natl Acad Sci USA 97: 883-888 
Murdoch JD, Rostosky CM, Gowrisankaran S, Arora AS, Soukup S-F, Vidal R, Capece V, Freytag S, Fischer A, Verstreken P, Bonn S, Raimundo N, Milosevic I (2016) Endophilin-A deficiency induces the Foxo3a-Fbxo32 network in the brain and causes dysregulation of autophagy and the ubiquitin-proteasome system. Cell Rep 17: 1071-1086

Neef J, Jung S, Wong AB, Reuter K, Pangrsic T, Chakrabarti R, Kugler S, Lenz C, Nouvian R, Boumil RM, Frankel WN, Wichmann C, Moser T (2014) Modes and regulation of endocytic membrane retrieval in mouse auditory hair cells. J Neurosci 34: 705-716

Neef J, Urban NT, Ohn T-L, Frank T, Jean P, Hell SW, Willig KI, Moser T (2018) Quantitative optical nanophysiology of $\mathrm{Ca}^{2+}$ signaling at inner hair cell active zones. Nat Commun 9: 290

Nouvian R, Neef J, Bulankina AV, Reisinger E, Pangršič T, Frank T, Sikorra S, Brose N, Binz T, Moser T (2011) Exocytosis at the hair cell ribbon synapse apparently operates without neuronal SNARE proteins. Nat Neurosci 14: $411-413$

Ohn T-L, Rutherford MA, Jing Z, Jung S, Duque-Afonso CJ, Hoch G, Picher MM, Scharinger A, Strenzke N, Moser T (2016) Hair cells use active zones with different voltage dependence of $\mathrm{Ca}^{2+}$ influx to decompose sounds into complementary neural codes. Proc Natl Acad Sci USA 113: E4716-E4725

Pangrsic T, Lasarow L, Reuter K, Takago H, Schwander M, Riedel D, Frank T, Tarantino LM, Bailey JS, Strenzke N, Brose N, Müller U, Reisinger E, Moser $T$ (2010) Hearing requires otoferlin-dependent efficient replenishment of synaptic vesicles in hair cells. Nat Neurosci 13: 869-876

Pangrsic T, Vogl C (2018) Balancing presynaptic release and endocytic membrane retrieval at hair cell ribbon synapses. FEBS Lett 592: 3633-3650

Parsons TD, Lenzi D, Almers W, Roberts WM (1994) Calcium-triggered exocytosis and endocytosis in an isolated presynaptic cell: capacitance measurements in saccular hair cells. Neuron 13: 875-883

Pechstein A, Gerth F, Milosevic I, Jäpel M, Eichhorn-Grünig M, Vorontsova O, Bacetic J, Maritzen T, Shupliakov O, Freund C, Haucke V (2015) Vesicle uncoating regulated by SH3-SH3 domain-mediated complex formation between endophilin and intersectin at synapses. EMBO Rep 16: 232-239

Perera RM, Zoncu R, Lucast L, Camilli PD, Toomre D (2006) Two synaptojanin 1 isoforms are recruited to clathrin-coated pits at different stages. Proc Natl Acad Sci USA 103: 19332-19337

Platzer J, Engel J, Schrott-Fischer A, Stephan K, Bova S, Chen H, Zheng H, Striessnig J (2000) Congenital deafness and sinoatrial node dysfunction in mice lacking class D L-type $\mathrm{Ca}^{2+}$ channels. Cell 102: 89-97

Ramakrishnan NA, Drescher MJ, Drescher DG (2009) Direct interaction of otoferlin with syntaxin 1A, SNAP-25, and the L-type voltage-gated calcium channel Cav1.3. J Biol Chem 284: 1364-1372

Reisinger E, Bresee C, Neef J, Nair R, Reuter K, Bulankina A, Nouvian R, Koch M, Bückers J, Kastrup L, Roux I, Petit C, Hell SW, Brose N, Rhee J-S, Kügler S, Brigande JV, Moser T (2011) Probing the functional equivalence of otoferlin and synaptotagmin 1 in exocytosis. J Neurosci 31: 4886-4895

Renard H-F, Simunovic M, Lemière J, Boucrot E, Garcia-Castillo MD, Arumugam S, Chambon V, Lamaze C, Wunder C, Kenworthy AK, Schmidt AA, McMahon HT, Sykes C, Bassereau P, Johannes L (2015) Endophilin-A2 functions in membrane scission in clathrin-independent endocytosis. Nature 517: $493-496$

Revelo NH, Kamin D, Truckenbrodt S, Wong AB, Reuter-Jessen K, Reisinger E, Moser T, Rizzoli SO (2014) A new probe for super-resolution imaging of membranes elucidates trafficking pathways. J Cell Biol 205: 591-606

Ringstad N, Gad H, Löw P, Paolo GD, Brodin L, Shupliakov O, Camilli PD (1999) Endophilin/SH3p4 is required for the transition from early to late stages in clathrin-mediated synaptic vesicle endocytosis. Neuron 24: $143-154$
Roux I, Safieddine S, Nouvian R, Grati M, Simmler M-C, Bahloul A, Perfettini I, Le Gall M, Rostaing P, Hamard G, Triller A, Avan P, Moser T, Petit C (2006) Otoferlin, defective in a human deafness form, is essential for exocytosis at the auditory ribbon synapse. Cell 127: 277-289

Roux I, Hosie S, Johnson SL, Bahloul A, Cayet N, Nouaille S, Kros C], Petit C, Safieddine $S$ (2009) Myosin VI is required for the proper maturation and function of inner hair cell ribbon synapses. Hum Mol Genet 18: 4615- 4628

Rutherford MA, Moser T (2016) The ribbon synapse between type I spiral ganglion neurons and inner hair cells. In The primary auditory neurons of the mammalian cochlea, Dabdoub A, Fritzsch B, Popper AN, Fay RR (eds), pp 117-156. Springer Handbook of Auditory Research. New York, NY: Springer

Safieddine S, El-Amraoui A, Petit C (2012) The auditory hair cell ribbon synapse: from assembly to function. Annu Reu Neurosci 35: 509-528

Saheki Y, Camilli PD (2012) Synaptic vesicle endocytosis. Cold Spring Harb Perspect Biol 4: a005645

Sanchez del Rio M, Pareschi G (2001) Global optimization and reflectivity data fitting for x-ray multilayer mirrors by means of genetic algorithms. In Proc. SPIE 4145, Aduances in X-Ray Optics. https://doi.org/10.1117/12.411624

Schuske KR, Richmond JE, Matthies DS, Davis WS, Runz S, Rube DA, van der Bliek AM, Jorgensen EM (2003) Endophilin is required for synaptic vesicle endocytosis by localizing synaptojanin. Neuron 40: 749-762

Shnerson A, Pujol R (1981) Age-related changes in the C57BL/6J mouse cochlea. I. Physiological findings. Deu Brain Res 2: 65-75

Siegel JH, Brownell WE (1986) Synaptic and Golgi membrane recycling in cochlear hair cells. J Neurocytol 15: 311-328

Simunovic M, Manneville J-B, Renard H-F, Evergren E, Raghunathan K, Bhatia D, Kenworthy AK, Voth GA, Prost J, McMahon HT, Johannes L, Bassereau P, Callan-jones A (2017) Friction mediates scission of tubular membranes scaffolded by BAR proteins. Cell 170: 172-184.e11

Sobkowicz HM, Rose JE, Scott GE, Slapnick SM (1982) Ribbon synapses in the developing intact and cultured organ of Corti in the mouse. J Neurosci 2: 942-957

Soda K, Balkin DM, Ferguson SM, Paradise S, Milosevic I, Giovedi S, VolpicelliDaley L, Tian X, Wu Y, Ma H, Son SH, Zheng R, Moeckel G, Cremona O, Holzman LB, De Camilli P, Ishibe S (2012) Role of dynamin, synaptojanin, and endophilin in podocyte foot processes. J Clin Invest 122: 4401-4411

Strenzke N, Chanda S, Kopp-Scheinpflug C, Khimich D, Reim K, Bulankina AV, Neef A, Wolf F, Brose N, Xu-Friedman MA, Moser T (2009) Complexin-I is required for high-fidelity transmission at the endbulb of Held auditory synapse. J Neurosci 29: 7991-8004

Strenzke N, Chakrabarti R, Al-Moyed H, Müller A, Hoch G, Pangrsic T, Yamanbaeva G, Lenz C, Pan K-T, Auge E, Geiss-Friedlander R, Urlaub H, Brose N, Wichmann C, Reisinger E (2016) Hair cell synaptic dysfunction, auditory fatigue and thermal sensitivity in otoferlin Ile515Thr mutants. EMBO J 35: 2519-2535

Sundborger A, Soderblom C, Vorontsova O, Evergren E, Hinshaw JE, Shupliakov O (2011) An endophilin-dynamin complex promotes budding of clathrin-coated vesicles during synaptic vesicle recycling. J Cell Sci 124: $133-143$

Trapani JG, Obholzer N, Mo W, Brockerhoff SE, Nicolson T (2009) Synaptojanin1 is required for temporal fidelity of synaptic transmission in hair cells. PLoS Genet 5: e1000480

Vehlow A, Soong D, Vizcay-Barrena G, Bodo C, Law A-L, Perera U, Krause M (2013) Endophilin, lamellipodin, and mena cooperate to regulate F-actindependent EGF-receptor endocytosis. EMBO J 32: 2722 - 2734

Verstreken P, Kjaerulff O, Lloyd TE, Atkinson R, Zhou Y, Meinertzhagen IA, Bellen HJ (2002) Endophilin mutations block clathrin-mediated endocytosis but not neurotransmitter release. Cell 109: 101-112 
Verstreken P, Koh T-W, Schulze KL, Zhai RG, Hiesinger PR, Zhou Y, Mehta SQ, Cao Y, Roos J, Bellen HJ (2003) Synaptojanin is recruited by endophilin to promote synaptic vesicle uncoating. Neuron 40: 733-748

Vincent PF, Bouleau Y, Petit C, Dulon D (2015) A synaptic F-actin network controls otoferlin-dependent exocytosis in auditory inner hair cells. Elife 4: e10988

Vogl C, Cooper BH, Neef J, Wojcik SM, Reim K, Reisinger E, Brose N, Rhee J-S, Moser T, Wichmann C (2015) Unconventional molecular regulation of synaptic vesicle replenishment in cochlear inner hair cells. J Cell Sci 128: $638-644$

Vogl C, Panou I, Yamanbaeva G, Wichmann C, Mangosing SJ, Vilardi F, Indzhykulian AA, Pangršič T, Santarelli R, Rodriguez-Ballesteros M, Weber T, Jung S, Cardenas E, Wu X, Wojcik SM, Kwan KY, Del Castillo I, Schwappach B, Strenzke N, Corey DP et al (2016) Tryptophan-rich basic protein (WRB) mediates insertion of the tail-anchored protein otoferlin and is required for hair cell exocytosis and hearing. EMBO J 35: $2536-2552$

Vogl C, Butola T, Haag N, Hausrat TJ, Leitner MG, Moutschen M, Lefèbvre PP, Speckmann C, Garrett L, Becker L, Fuchs H, Hrabe de Angelis M, Nietzsche
S, Kessels MM, Oliver D, Kneussel M, Kilimann MW, Strenzke N (2017) The $B E A C H$ protein $L R B A$ is required for hair bundle maintenance in cochlear hair cells and for hearing. EMBO Rep 18: 2015-2029

Watanabe S, Mamer LE, Raychaudhuri S, Luvsanjav D, Eisen J, Trimbuch T, Söhl-Kielczynski B, Fenske P, Milosevic I, Rosenmund C, Jorgensen EM (2018) Synaptojanin and endophilin mediate neck formation during ultrafast endocytosis. Neuron 98: 1184-1197.e6

Weiler S, Krinner S, Wong AB, Moser T, Pangršič T (2014) ATP hydrolysis is critically required for function of CaV1.3 channels in cochlear inner hair cells via fueling $\mathrm{Ca}^{2+}$ clearance. J Neurosci 34: 6843-6848

Wichmann C, Moser T (2015) Relating structure and function of inner hair cell ribbon synapses. Cell Tissue Res 361: 95-114

Wong AB, Rutherford MA, Gabrielaitis M, Pangršič T, Göttfert F, Frank T, Michanski S, Hell S, Wolf F, Wichmann C, Moser T (2014) Developmental refinement of hair cell synapses tightens the coupling of $\mathrm{Ca}^{2+}$ influx to exocytosis. EMBO J 33: 247-264

Yang Y, Wei M, Xiong Y, Du X, Zhu S, Yang L, Zhang C, Liu J-J (2015) Endophilin $A 1$ regulates dendritic spine morphogenesis and stability through interaction with p140Cap. Cell Res 25: 496-516 


\section{Expanded View Figures}

A

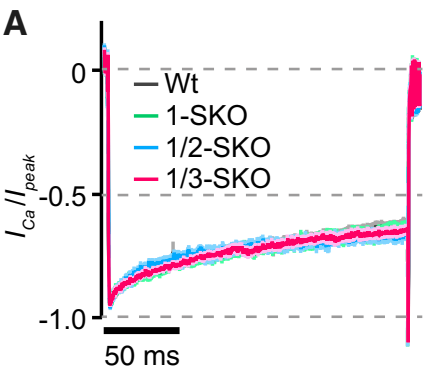

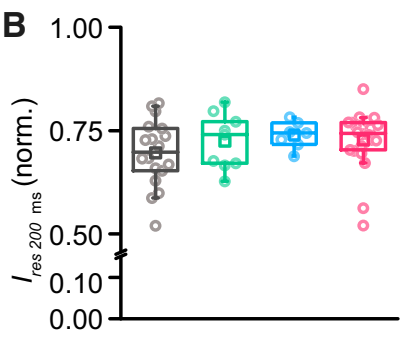

A
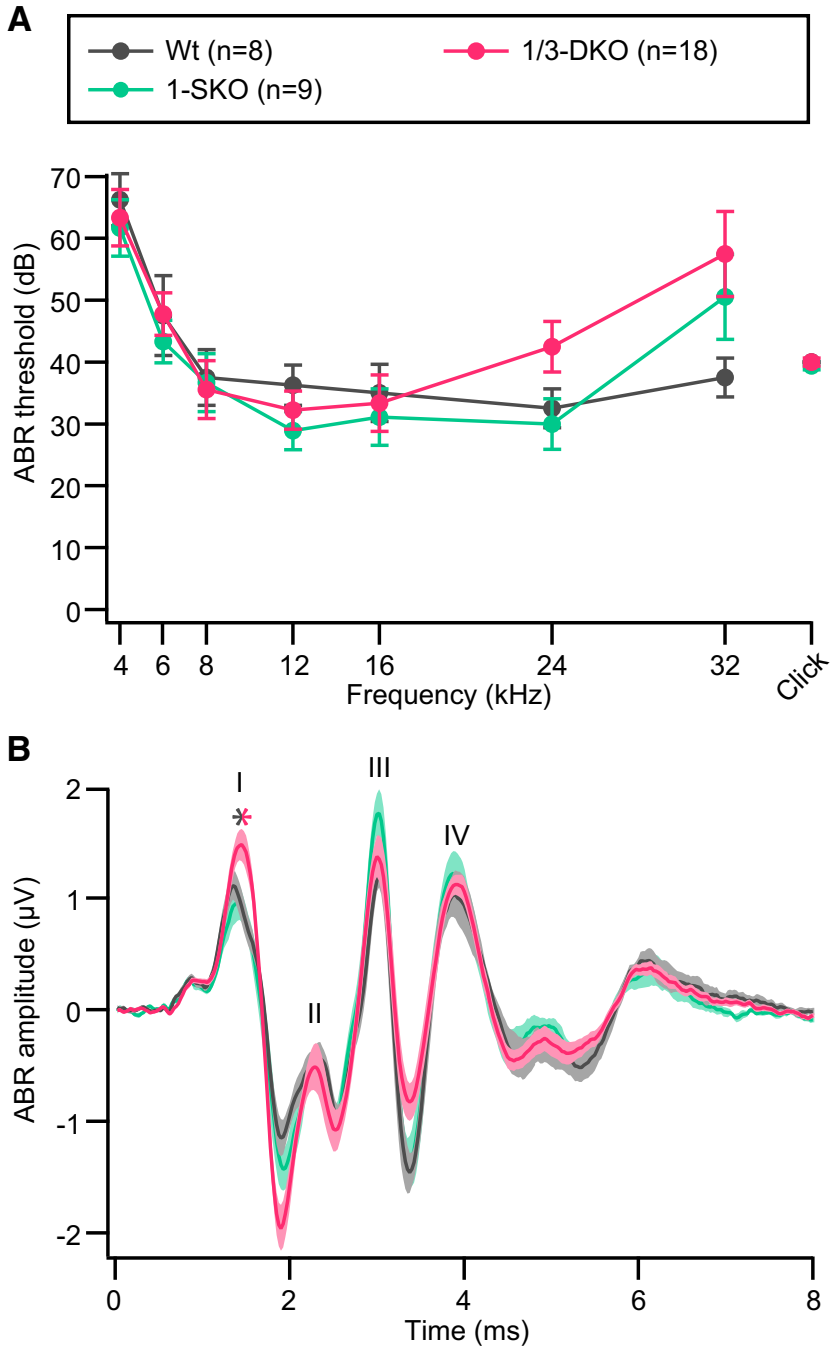

Figure EV1. $\mathrm{Ca}^{2+}$ inactivation remains unaffected in endophilindeficient IHCs after hearing onset.

A $\mathrm{Ca}^{2+}$ current inactivation was probed by test pulses of $200 \mathrm{~ms}$ to the $I_{\max }$ potential. No inactivation phenotype was present in endophilin-deficient IHCS. Data represent grand averages \pm SEM.

B Quantification and statistical analysis of the residual current at the end of the test pulse. (Wt $n=18 ; 1$-SKO $n=9 ; 1 / 2-$ DKO $n=7 ; 1 / 3$-DKO $n=22$; one-way ANOVA; box plots illustrate the median with the interquartile range, whiskers indicate $10-90 \%$ of data points, and the squares present the respective mean value).
Figure EV2. Normal auditory brainstem responses (ABRs) in 1-SKO and 1/ 3-DKO mice.

A Sound thresholds of ABRs of 6-week-old 1-SKO, 1/3-DKO, and Wt mice. Thresholds are displayed versus tone frequency or click (broadband). Error bars indicate the SEM. For (A) and (B), the following numbers of animals were tested: Wt $N=8 ; 1-$ SKO $N=9 ; 1 / 3-$ DKO $N=18$.

B ABR waveforms elicited by $80 \mathrm{~dB}$ clicks in mice of the respective genotypes. Roman numbers indicate the peaks of Jewett Waves I-IV. Data represent grand averages ( \pm SEM) of the ABRs, whereby each animal contributed the average of 1,000 responses to the click. One-way ANOVA followed by post hoc Tukey's test; ${ }^{*} P=0.0134$. 

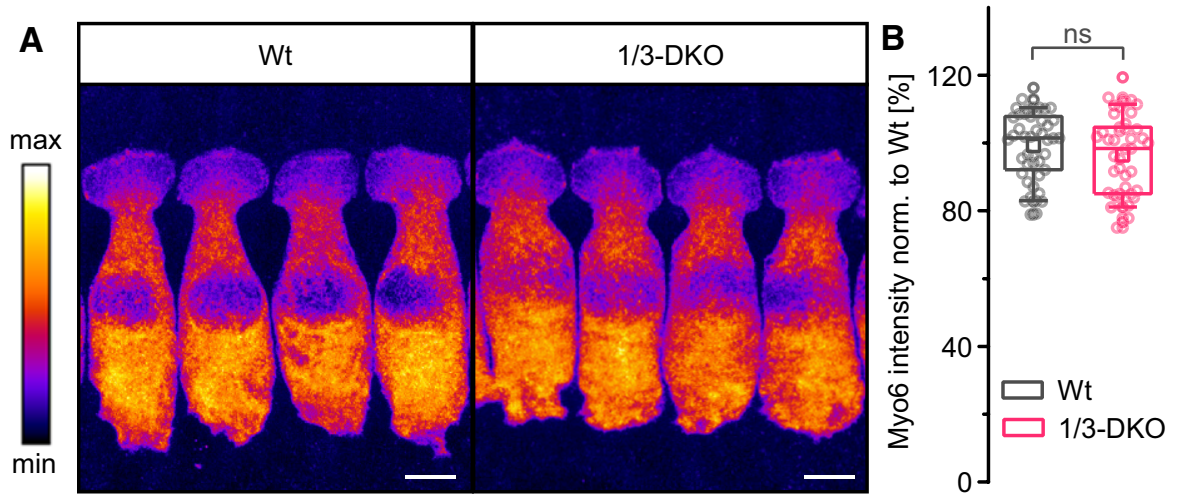

Figure EV3. Myosin 6 protein levels are unaltered in the absence of endophilins.

A Confocal maximum projections of myo6immunolabeled organs of Corti from p16 Wt and 1/3-DKO mice illustrated with an intensity-coded lookup table. Scale bars: $5 \mu \mathrm{m}$.

B Myo6 levels were unaltered in 1/3-DKO IHCS compared to Wt IHCS (relative intensities normalized to $\mathrm{Wt}$; mean \pm SEM; Wt $n=6$ images ( $45 \mathrm{IHCS}) / \mathrm{N}=6$ organs of Corti (three animals); 1/3-DKO $n=6$ images $(43 \mathrm{IHCs}) / N=6$ organs of Corti (three animals); unpaired Student's $t$-test; box plots illustrate the median with the interquartile range, whiskers indicate $10-90 \%$ of data points, and the squares present the respective mean value).

\section{Figure EV4. Methods to correct for the depolarization-independent decrease of $C_{m}$ in endocytosis measurements.}

A Representative example of the slow depolarization-independent decrease of baseline $C_{m}$ (circles) during a perforated patch-clamp recording of one Wt IHC. This decline can be fitted by an exponential function (black trace).

B Uncorrected 20-ms depolarization traces recorded from one Wt IHC. Traces are color-coded based on the order of acquisition, corresponding to the colormatching circles in panel (A).

C Method 1 to correct for the stimulus-independent decrease in baseline $C_{m}$ : The fitted exponential function from panel (A) was subtracted from the individual raw traces. The average trace is shown in black.

$C^{\prime}-C^{\prime \prime \prime} \quad$ Results obtained after performing the correction method 1 and fitting a linear function to the $C_{m}$ data following a 20-ms depolarization: $\left(C^{\prime}\right)$ average $\Delta C_{m}$ recordings ( \pm SEM) for Wt, 1/2-DKO, and 1/3-DKO IHCS, $\left(C^{\prime \prime}\right)$ average time of return to baseline, and $\left(\mathrm{C}^{\prime \prime \prime}\right)$ average slope of the linear component of endocytosis. ${ }^{*} P<0.05$; one-way ANOVA with post hoc Tukey's test. Please also refer to Appendix Table $\mathrm{S8}$ for detailed statistical analysis.

D Method 2 to correct for the stimulus-independent decrease of baseline $\mathrm{C}_{\mathrm{m}}$ : A linear function was fitted to the last $10 \mathrm{~s}$ of a non-depolarizing pulse recorded before the actual trace. The fit was subtracted from each corresponding raw trace. The average trace is shown in black.

$D^{\prime}-D^{\prime \prime \prime} \quad$ Results obtained after performing the correction method 2 and fitting a linear function to the $C_{m}$ data following a 20-ms depolarization: $\left(D^{\prime}\right)$ average $\Delta C_{m}$ recordings ( \pm SEM) for Wt, 1/2-DKO, and 1/3-DKO IHCS, $\left(D^{\prime \prime}\right)$ average time of return to baseline, and ( $\left.\mathrm{D}^{\prime \prime \prime}\right)$ average slope of the linear component of endocytosis. ${ }^{*} P<0.05$; one-way ANOVA with post hoc Tukey's test: $P=0.0206$ for Wt versus $1 / 2$-DKO and $P=0.0274$ for Wt versus $1 / 3$-DKO.

Data information: Box plots in panels ( $\left(C^{\prime \prime}-\mathrm{D}^{\prime \prime \prime}\right)$ illustrate the median with the interquartile range, whiskers indicate $10-90 \%$ of data points, and the squares present the respective mean value. 

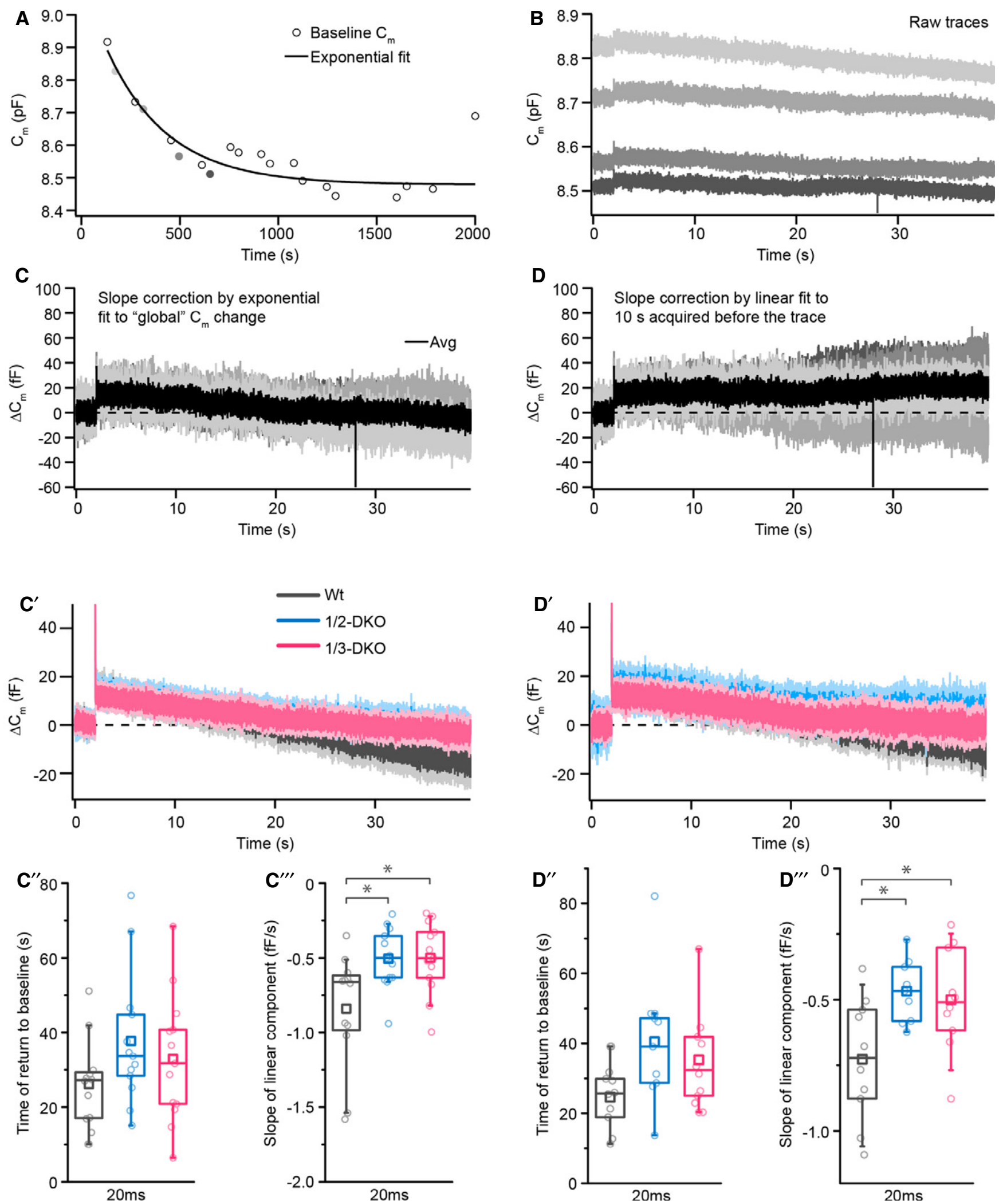

Figure EV4. 
CHAPTER TWO 


\subsection{GENERAL INFORMATION}

TITLE: AP180 PROMOTES RELEASE SITE CLEARANCE AND CLATHRIN-DEPENDENT VESICLE REFORMATION IN MOUSE COCHLEAR INNER HAIR CELLS

AUTHORS:

Jana Kroll 1,2,3,4,* , Özge Demet Özçete ${ }^{3,45,6,{ }^{*},}$, Sangyong Jung ${ }^{5,7}$, Tanja M aritzen ${ }^{8}$, Ira M ilosevic ${ }^{1,3}$, Carolin Wichmann ${ }^{2,3,5, \#, \text { Tobias Moser } 3,3,6,9, \#}$

${ }^{1}$ Synaptic Vesicle Dynamics Group, European N euroscience Institute Göttingen - A Joint Iniciative of the University M edical Center Göttingen and the M ax-Planck-Society, Göttingen, Germany

${ }^{2} \mathrm{M}$ olecular A rchitecture of Synapses G roup, Institute for Auditory N euroscience, InnerEarLab and Center for Biostructural Imaging of Neurodegeneration, University M edical Center Göttingen, Göttingen, Germany

${ }^{3}$ Collaborative Research Center 889, U niversity of Göttingen, Göttingen, Germany

${ }^{4} \mathrm{Gött} i n g e n$ Graduate School for N euroscience and M olecular Biosciences, University of Göttingen, Göttingen, Germany

IInstitute for Auditory Neuroscience and InnerEarLab, University Medical Center Göttingen, Göttingen, Germany

${ }^{6}$ Auditory Neuroscience Group, Max Planck Institute for Experimental Medicine, Göttingen, Germany

${ }^{7}$ Neuro Modulation and Neuro Circuitry Group, Singapore Bioimaging Consortium (SBIC), Biomedical Sciences Institutes, 138667 Singapore

${ }^{8}$ Leibniz-Forschungsinstitut für M olekulare Pharmakologie (FM P), Berlin, Germany

'Synaptic Nanophysiology Group, Max Planck Institute for Biophysical Chemistry, Göttingen, Germany

*these authors contributed equally to this work "corresponding authors

The electrophysiological experiments and systems physiology analysis performed by Özge Demet Özçete, former PhD student at the Institute for A uditory N euroscience Göttingen, is not included in this chapter, but is part of the manuscript published in the Journal of Cell Science.

PUBLICATION PROGRESS:

- Submitted to Journal of Cell Science $17^{\text {th }}$ July 2019

- Resubmitted after revision $15^{\text {th }}$ November 2019

- Accepted $9^{\text {th }}$ December 2019 
MY CONTRIBUTIONS TO THE MANUSCRIPT:

MY CONTRIBUTIONS

FIG. 3-1 O Immunohistochemical staining (AP180, CtBP2, Otoferlin, Synapsin1/2) and confocal microscopy

- Panel $B_{i i}$ : Intensity profile line scan analysis using ImageJ software

○ Figure design

FIG. 3-2 $\quad$ Immunohistochemical staining of otoferlin and AP180 after $\mathrm{K}^{+}$stimulation and confocal microscopy

- Panel C- $\mathrm{C}_{\mathrm{ii}}$ : Intensity profilelinescan analysis using I mageJ software, statistics

- Panel $D-D_{i}:$ High-intensity cluster analysis using I mageJ software, statistics

- Figure design

FIG. 3-3 O Immunohistochemical staining (Otoferlin + Vglut3, CtBP2 + Homer1) and confocal microscopy

- Panels $A_{i}$ and $B_{i}$ : Otoferlin and V glut3 fluorescence intensity analysis using Imaris and Matlab (as described for otoferlin in the endophilin study), statistics

- Panel $C_{i}: M$ anual quantification of ribbon synapses using imagel software

- Figure design

FIG.3-4 O Sample preparation including HPF, freeze substitution, sectioning and staining

- Electron tomography, reconstruction of tomograms, analysis using IM OD software, statistics

- Figure design

- Sample preparation (with $\mathrm{K}^{+}$stimulation), electron tomography, analysis and statistics like for Fig. 3-4

FIG. 3-6 Figure design

MANUSCRIPT W riting of the here presented manuscript draft (edited by T.M . and C.W.) 


\subsection{MANUSCRIPT}

\subsubsection{ABSTRACT}

High-throughput neurotransmission at ribbon synapses of cochlear inner hair cells (IHCs) requires tight coupling of neurotransmitter release and balanced recycling of synaptic vesicles (SV s) as well as rapid restoration of release sites. Here, we examined the role of the adaptor protein AP180 (also known as SNAP91) for IHC synaptic transmission by comparing AP180-knockout (KO) and wildtype mice using high-pressurefreezing and electron tomography, as well as confocal microscopy. A P180 was found predominantly at the synaptic pole of IHCS. A P180-deficient IHCs had severely reduced SV numbers and accumulated endocytic intermediates near ribbon synapses, indicating that AP180 is required for clathrin-dependent endocytosis and SV reformation in IHCS. Moreover, A P180 deletion led to a high prevalence of SV s in a multi-tethered or docked state after stimulation. We conclude that, in addition to its role in clathrin recruitment, AP180 contributes to release site clearance in IHCS. 


\subsubsection{INTRODUCTION}

Ribbon synapses of inner hair cells (IHCs) of the cochlea with spiral ganglion neurons feature one of the highest rates of continuous neurotransmitter release in the mammalian body in order to ind efatigably encode sound (Pangršič et al., 2012). H ence, hearing requires on the one hand the very fast exocytosis of neurotransmitter-containing SVs and on the other hand a highly efficient endocytosis and reformation of fusion-competent synaptic vesicles (SV s) to balance the massive SV exocytosis. The specialized structure of the IHC activezone (AZ) - notably the electron-dense ribbon that is made of RIBEYE (an isoform of CtBP2, herein denoted RIBEYE/CtBP2) and tethers a halo of SV s (Becker et al., 2018; Jean et al., 2018; Khimich et al., 2005; Schmitz et al., 2000) - and a unique protein composition of release machinery and AZ cytomatrix are thought to accomplish these tasks (reviewed in: M oser et al., 2019; Rutherford and M oser, 2016; Safieddine et al., 2012; W ichmann and Moser, 2015). Interestingly, IHC exocytosis seems to operate without the neuronal soluble Nethylmaleimide-sensitive-factor attachment receptor (SNARE) proteins SNAP25, syntaxin-1 and VAM P2 (N ouvian et al., 2011). Instead, the multi- $C_{2}$ domain protein otoferlin, loss of which causes human genetic deafness (Yasunaga et al., 1999), serves as a key molecule in IHC exocytosis. A fter the onset of hearing in mice, otoferlin is thought to be the $\mathrm{Ca}^{2+}$-sensor of exocytosis in $\mathrm{IHCS}$, and also to mediate efficient SV replenishment, possibly by advancing SV tethering and release site clearance (Chakrabarti et al., 2018; Duncker et al., 2013; Johnson and Chapman, 2010; Jung et al., 2015b; K roll et al., 2019; Michalski et al., 2017; Pangršič et al., 2010; Roux et al., 2006; Strenzke et al., 2016; Vogl et al., 2015, 2016). Clearance of previously exocytosed proteins and lipids from the release sites is considered a critical step in preparing the next round of SV exocytosis (H aucke et al., 2011; Neher and Sakaba, 2008). Otoferlin's action in release site clearance is likely achieved through binding to the adaptor protein 2 (AP-2) (Duncker et al., 2013; Jung et al., 2015b) and/or endophilin A 1 (K roll et al., 2019). Like in neurons (Kononenko et al., 2014), absence of AP-2 from IH Cs compromises the clathrin-dependent reformation of SV s from endosome-like vacuoles (ELVs), but, surprisingly, does not significantly impair endocytic membrane retrieval (Jung et al., 2015b).

Endocytic membrane retrieval in IHCS comprises a slow, linear component that likely reflects clathrin-mediated endocytosis (CME), and a fast, exponential component thought to represent bulk retrieval (M oser and Beutner, 2000; N eef et al., 2014). M orphological evidence for CME and bulk retrieval in hair cells has been reported previously (e.g., Jung et al., 2015b; Lenzi et al., 2002; N eef et al., 2014; Siegel and Brownell, 1986). Molecular entities identified to mediate SV recycling in hair 
cells include dynamins, endophilin-As, clathrin and synaptojanin-1 (Boumil et al., 2010; Jung et al., 2015b; Kroll et al., 2019; Neef et al., 2014; Trapani et al., 2009). Their disruption primarily affected the slow component of IHC membrane retrieval, i.e. CME. So far, little is known about whether the molecular regulation of bulk retrieval at hair cell synapses is similar to that described for other synapses and neuroendocrine cells (for review see, e.g. Cousin, 2017; Wu et al., 2014)

A further dissection of the molecular physiology of IHC SV recycling is key to comprehending indefatigable sound encoding. In this context, the neuronal endocytic adaptor protein AP180 is an interesting candidate protein. Through binding of clathrin, AP-2 and the phospholipid phosphatidylinositol 4,5-bisphosphate [PI $\left.(4,5) \mathrm{P}_{2}\right]$, AP180 was shown to be involved in the early steps of clathrin-coated pit (CCP) formation in neurons (Ford et al., 2001; H ao et al., 1999; Lindner and Ungewickell, 1992; M orris et al., 1993; M oshkanbaryans et al., 2016). In AP180 knock-out (AP180KO) mice, accumulations of ELVs and lower numbers of clathrin-coated structures have been observed, underlining the importance of AP180 for CME as well as for clathrin-dependent SV reformation from ELVs (Koo et al., 2015). Through its AP180 N-terminal homology (ANTH) domain, AP180 binds to the SNARE domain of VAMP2 and other members of the VAM P family (Koo et al., 2011; Maritzen et al., 2012). This way, AP180, as well as its ubiquitously expressed homolog CALM, organize the recycling of VAMP2 from the plasma membrane after SNARE complex disassembly following SV fusion and, thereby, contribute to the clearance of release sites in neurons (Koo et al., 2011, 2015; M aritzen et al., 2012; M iller et al., 2011). AP180-dependent clearance of release sites seems to be of greater importance in inhibitory neurons that show higher rates of transmitter release than excitatory neurons (Koo et al., 2015). H owever, the relevance of AP180 for high-throughput synaptic transmission of sensory ribbon synapses is unclear, especially at IHCS, which seem to operate without the AP180 cargo protein VAM P2 (N ouvian et al., 2011).

Here, we studied the role of AP180 at murine IHC ribbon synapses by combining confocal microscopy, chemical depolarization of $\mathrm{IHCS}$, high-pressure freezing and electron tomography. We discovered that AP180 has a role in release site clearance, clathrin assembly for CME, as well as SV reformation following bulk retrieval in IHCS. 


\subsubsection{METHODS}

ANIMALS

Constitutive knockout mice for AP180 as well as their wildtype littermates, previously described in (Koo et al., 2015) were used in this study. For all experiments, mice of either sex were examined shortly after the onset of hearing (P15-P16). For the analysis of AP180 localization and distribution, C57BL6/J mice were used. All experiments complied with national animal care guidelines and were approved by the University M edical Center Göttingen board for animal welfare and the animal welfare office of the state of Lower Saxony.

\section{IMMUNOHISTOCHEMISTRY AND CONFOCAL MICROSCOPY}

Freshly dissected apical turns of organs of Corti from two-weeks old mice were (i) chemically stimulated for 1 min using high- $\mathrm{K}^{+}$stimulation solution $(65.36 \mathrm{mM} \mathrm{KCl}, 79.7 \mathrm{mM} \mathrm{NaCl}, 2 \mathrm{mM}$ $\mathrm{CaCl}_{2}, 1 \mathrm{mM} \mathrm{M} \mathrm{gCl} 2,0.5 \mathrm{mM} \mathrm{M} \mathrm{gSO}_{4}, 10 \mathrm{mM}$ HEPES, $3.4 \mathrm{mM}$ L-glutamine, and $6.9 \mathrm{mM}$ D-glucose, $\mathrm{pH}$ 7.4) or incubated in a high $\mathrm{Ca}^{2+}$, low $\mathrm{K}^{+}$solution for control $(5.36 \mathrm{mM} \mathrm{KCl}, 139.7 \mathrm{mM} \mathrm{NaCl}, 2$ $\mathrm{mM} \mathrm{CaCl}, 1 \mathrm{mM} \mathrm{MgCl} 2,0.5 \mathrm{mM} \mathrm{MgSO}{ }_{4}, 10 \mathrm{mM}$ HEPES, $3.4 \mathrm{mM}$ L-glutamine, and $6.9 \mathrm{mM}$ D-glucose, pH 7.4; also see (Revelo et al., 2014) at room temperature followed by fixation using 4\% formaldehyde (FA) in phosphate buffered saline (PBS) on ice, or (ii) were directly fixed with $4 \% \mathrm{FA}$ in PBS for 10 min (for RIBEYE/CtBP2 and Homer1) or for 60 min (for otoferlin, V glut3 and AP180) on ice. After 3x10 min washing in PBS, blocking solution (goat serum dilution buffer (GSDB); 16\% normal goat serum, $450 \mathrm{mM} \mathrm{NaCl}, 0.3 \%$ Triton X-100, $20 \mathrm{mM}$ phosphate buffer, pH 7.4) was applied for $1 \mathrm{~h}$ in a wet chamber at room temperature. Primary antibodies were diluted in GSD B and applied overnight at $4^{\circ} \mathrm{C}$ in a wet chamber. After three 10 min washes (wash buffer: $450 \mathrm{mM} \mathrm{NaCl}, 20 \mathrm{mM}$ phosphate buffer, $0.3 \%$ Triton X-100), secondary antibodies diluted in GSD B were applied in a lightprotected wet chamber for $1 \mathrm{~h}$ at room temperature. Then, the specimens were washed three times in wash buffer and finally in $5 \mathrm{mM}$ phosphate buffer and mounted onto glass microscope slides with mounting medium (M owiol 4-88, Sigma). The following primary antibodies were used: rabbit antiAP180 (1:300, Synaptic Systems, cat. N o. 155 003), mouse anti-CtBP2 (also recognizing the ribbon protein ribeye, 1:200, BD Biosciences, cat. N o. 612044), mouse anti-otoferlin (1:300, A bcam, cat. No. ab53233), rabbit anti-V glut3 (1:300, Synaptic Systems, cat. N o. 135 203), rabbit anti-H omer1 (1:200, Synaptic Systems, cat. N o. 160 002) and guinea pig anti-synapsin1/2 (Synaptic Systems, cat. No. 106 
002). The secondary antibodies used for confocal microscopy were goat anti-rabbit-IgG conjugated to AlexaFluor488 (1:200, Invitrogen, cat. No. A 11008), goat anti-mouse-IgG conjugated to A lexaFluor568 (1:200, Invitrogen, cat. No. A 11004), goat anti-mouse-IgG conjugated to A lexaFluor633 (1:200, Invitrogen, cat. No. A 21136), and goat anti-guinea pig-IgG conjugated to A lexaFluor568 (1:200, Invitrogen, cat. No. A 11075). Confocal images were acquired using a laser scanning confocal microscope (Zeiss LSM 800, Carl Zeiss AG, O berkochen, Germany) with $488 \mathrm{~nm}$ (A r) and $561 \mathrm{~nm}$ ( $\mathrm{He}-\mathrm{Ne}$ ) lasers for excitation and 1.4 NA 63x oil immersion objectives. Z-axis stacks of $2 \mathrm{D}$ images were generated with a step size of $0.6 \mu \mathrm{m}$ from comparable tonotopic regions. Images were processed using Image) (http://imagej.net/) and assembled for display in Adobe Illustrator software. For semiquantitative analysis of immunofluorescence, samples of A P180-KO and control/ unstimulated and stimulated probes were processed identically and in parallel throughout immunohistochemistry, confocal imaging, and analysis.

\section{High-PreSSURe FreEZING AND FreEZe SUbSTitution}

HPF and FS were essentially performed as described previously (Chakrabarti et al., 2018; Jung et al., 2015a; W ong et al., 2014). Briefly, for H PF, the apical cochlear turns from P15-P16 Wt and AP180$\mathrm{KO}$ mice were dissected in $\mathrm{Ca}^{2+}$-free $\mathrm{H}$ anks' balanced salt solution (HBSS) and carefully placed on aluminium specimen carriers in the $0.2 \mathrm{~mm}$ cavity (type $A, 0.1 \mathrm{~mm}$ and $0.2 \mathrm{~mm}$ cavity, Leica M icrosystems, W etzlar, Germany) filled with HBSS solution. A second specimen carrier (0.3 mm cavity, type B, Leica M icrosystems) was dipped in hexadecene and placed onto the first specimen carrier with the cavity upwards. An EM HPM 100 (Leica M icrosystems) high-pressure freezer was used for sample freezing. I mmediately afterwards, samples were transferred into liquid nitrogen and stored until freeze substitution was performed. For stimulation, organs of Corti were dissected in $\mathrm{Ca}^{2+}$-free HBSS solution and transferred into stimulation solution (identical with the solution used for immunohistochemistry experiments). Within the stimulation solution, samples were placed on specimen carriers and frozen exactly 5 min after the transfer.

Freezesubstitution was performed with an EM AFS2 (Leica M icrosystems) freeze substitution device. Organs of Corti were transferred into the AFS2 precooled to $-90^{\circ} \mathrm{C}$. After $4 \mathrm{~d}$ incubation in $0.1 \%$ tannic acid in acetone, samples were washed threetimes for $1 \mathrm{~h}$ each time in acetone before $2 \%(\mathrm{w} / \mathrm{v})$ osmium tetroxide in acetone was applied at $-90^{\circ} \mathrm{C}$. The temperature was increased from $-90^{\circ} \mathrm{C}$ to $-20^{\circ} \mathrm{C}\left(5^{\circ} \mathrm{C} / \mathrm{h}\right)$, maintained at $-20^{\circ} \mathrm{C}$ for $17 \mathrm{~h}$ and was further increased from $-20^{\circ} \mathrm{C}$ to $+4^{\circ} \mathrm{C}$ 
$\left(10^{\circ} \mathrm{C} / \mathrm{h}\right) .0$ smium tetroxide was removed at $4^{\circ} \mathrm{C}$ and samples were washed three times for $1 \mathrm{~h}$ each time with acetone. Subsequently, samples were slowly warmed to room temperature. Finally, organs of Corti were infiltrated with Epon resin (acetone/Epon 1:1 (v/v) for 2h, 100\% Epon overnight), placed into embedding molds and polymerized for $48 \mathrm{~h}$ at $70^{\circ} \mathrm{C}$.

\section{ELECTRON TOMOGRAPHY}

Electron tomography of ribbon synapses was performed as described previously (Jung et al., 2015b; Strenzke et al., 2016). $250 \mathrm{~nm}$ sections of the embedded samples were obtained approaching from the anterior edge using an ultramicrotome (UC6, Leica M icrosystems, Wetzlar, Germany) with a $35^{\circ}$ diamond knife (Diatome, Nidau, Switzerland) and applied to formvar-coated copper 75-mesh grids. Sections were post-stained with uranyl acetate replacement (Science services, M unich, Germany) for $40 \mathrm{~min}$ and lead citrate for $1 \mathrm{~min}$ following standard protocols. To both sides of the grids, $10 \mathrm{~nm}$ gold beads (British Bio Cell, Crumlin, UK) were applied as fiducial markers. U sing Serial-EM software, single tilt series from $-60^{\circ}$ to $+60^{\circ}$ (increment $1^{\circ}$ ) were acquired with a JEM 2100 (JEOL, Freising, Germany) electron microscope at $200 \mathrm{kV}$ and 10,000x magnification. For tomogram generation, the IM OD package etomo was used, models were generated with 3dmod (bio3d.colorado.edu/imod/).

\section{DATA ANALYSIS}

Immunohistochemistry. The intensities of AP180, otoferlin and synapsin1/2 fluorescence signals along the longitudinal axis of IHCs (Fig. 3-1) were measured via line profiles using I mage) software. Igor Pro 6.3 software was used to generate average intensity profiles. The distribution of AP180 in the cytosol and in membrane proximity was assessed from intensity line scans reaching from the basal plasma membrane towards the cytosol along the longitudinal axis of IHCs (Fig. 3-2C- $\mathrm{C}_{\mathrm{ii}}$ ) and from a high-intensity fluorescence cluster analysis (Fig. 3-2D-Di ): Using Image software, middle planes of IH Cs were selected and cell borders were defined via otoferlin co-staining. In the otoferlin channel, lines from the cell membrane towards the nucleus were drawn and fluorescence intensity was measured in the AP180 channel (pixel intensity values ranging from 0 to 255) to avoid bias. I gor Pro 6.3 software was used to generate average intensity profiles of the individual cells. Separated clusters of high AP180 immunofluorescence intensity were defined by application of an intensity threshold (the threshold was adjusted once for clusters in a stimulated example image and then used for all images) using ImageJ software. Within each IHC (as defined by otoferlin co-staining), the 
average size and mean intensity of all clusters were calculated using the ImageJ tool "analyze particles". Only clusters with a size of $\geq 0.04 \mu \mathrm{m}^{2}$ were included because of the resolution limit of confocal microscopy (approx. 200-250 nm for the here used fluorophores). IHC ribbon synapses were manually counted in z-projections of confocal sections from RIBEYE/CtBP2 and Homer1 immunolabeled organs of Corti using Imagel software. Otoferlin and Vglut3 levels of IHCs were semi-quantitatively assessed as immunofluorescence intensity values and analyzed using Imaris (Bitplane) and M atlab (M athworks), as described previously (Strenzkeet al., 2016). Intensities of each cell are normalized to the average intensity of the Wt group for otoferlin or for $\mathrm{V}$ glut3.

Electron tomography. Only tomograms with a single ribbon synapse clearly anchored to the AZ were selected for quantification using the IMOD package 3dmod (bio3d.colorado.edu/imod/) and as described previously (Chakrabarti et al., 2018; Kroll et al., 2019). Small, clear vesicles were classified as synaptic vesicles (SV s) if they appeared round and if their outer diameter (defined by fitting a circle to the borders at the maximum projection of the respective SV) was smaller than $70 \mathrm{~nm}$. SV s were categorized as belonging to one of three groups: (i) ribbon-associated SV S (RA-SV s), if they were located in the first row around thesynaptic ribbon and with a max. distance of $80 \mathrm{~nm}$ from the ribbon, (ii) membrane-proximal SV s (M P-SVs) if the distance between SV and plasma membrane was less than $50 \mathrm{~nm}$ and if the distance between SV and presynaptic density was less than $100 \mathrm{~nm}$, and (iii) cytosolic SV s if the distance between SV and ribbon was less than $500 \mathrm{~nm}$ thereby excluding RA-SV s and MP-SV s (all criteria being valid at maximum projection of the respective SV). M P-SV s were further subdivided into four groups depending on their connection to the AZ plasma membrane as described in a previous study (Chakrabarti et al., 2018): SV s were counted as docked, if the distance between SV outer membrane and AZ membrane was 0-2 nm. Single-tethered SV s were defined as all SV s with only one tether reaching from theSV to theA Z membrane. M ulti-tethered SV swere defined as all SV s with more than one tether from the SV to the AZ membrane. SV s were categorized as untethered if no filament was observed between SV and plasma membrane (thereby ignoring possible filamentous connections between SV s, as well as between SV s and presynaptic density or between SV and ribbon). Furthermore, all structures containing a clathrin-coat were counted as coated structures if the minimal distance between organelle and ribbon was less than $500 \mathrm{~nm}$. This quantification includes structures with a coat around the entire clathrin-coated SV (independent of its size), coated pits budding from the plasma membrane, and endosome-like vacuoles (ELVs) with at least one budding coated pit (in this case, theELV with pit was only counted onceand not included 
in the additional ELV quantification). All membranous organelles were defined as ELV s and counted, if their max. diameter was larger than $70 \mathrm{~nm}$ and/or if they were tubular, and if the minimal distance between ELV and ribbon was less than $500 \mathrm{~nm}$. The volume of each ELV was assessed from a modelled mesh calculated from contours drawn in every virtual section of the ELV in 3dmod.

Statistical analysis. Sample sizes were chosen according to typical observation numbers used on each respective field and can be found in the respective figures and/or corresponding figure legends. For quantification, the following software was used: $M$ atlab (M athworks), Excel (M icrosoft), I gor Pro 6 (W avemetrics), Origin 9.0 (M icrocal Software) and GraphPad Prism (GraphPad Software). A verages are expressed as mean \pm SEM , boxes in box plots show the 25 - 75 quartiles with the median, whiskers extend from the $10^{\text {th }}-90^{\text {th }}$ percentile; the mean is indicated by a square or cross. Data sets were tested for normal distribution (D’Agostino \& Pearson omnibus normality test with GraphPad Prism software) and equality of variances (F-test). Statistical significance was calculated using unpaired, two-tailed student's t-test for normally distributed data, Mann-W hitney $U$ test for non-normally distributed data and Kolmogorov-Smirnov test to compare data distribution. For Fig. 3-5G, one-way ANOVA followed by Tukey's post-hoc test was used to compare multiple groups. Significant differences are reported as ${ }^{*} p<0.05,{ }^{* *} p<0.01$ and ${ }^{* * *} p<0.001$.

\subsubsection{RESULTS}

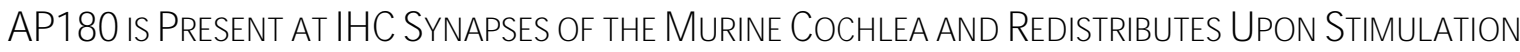

First, we aimed to identify if the adaptor protein AP180 is present in the murine organ of Corti, and, more specifically, in IHCs. Therefore, we performed immunohistochemistry in apical cochlear turns ( 2-12 kHz) of wildtype mice (Wt) acutely explanted after the onset of hearing, as well as of agematched AP180-KO mice (used as a control for antibody specificity). We immunostained for AP180 in combination with markers for efferent synapses (synapsin1/2; note that IHCS do not contain synapsins 1 and 2), the synaptic ribbon (RIBEYE/CtBP2), and hair cells (otoferlin). Immunofluorescence (Fig. 3-1A left panel) revealed the presence of AP180 in IHCS and outer hair cells (OHCs), as well as in efferent synapses underneath the hair cells and possibly in spiral ganglion neurons (see their somata in the inset to Fig. 3-1A). A lack of A P180 staining in organs of Corti from AP180-KO mice confirmed the high specificity of the AP180 antibody (Fig. 3-2A). 


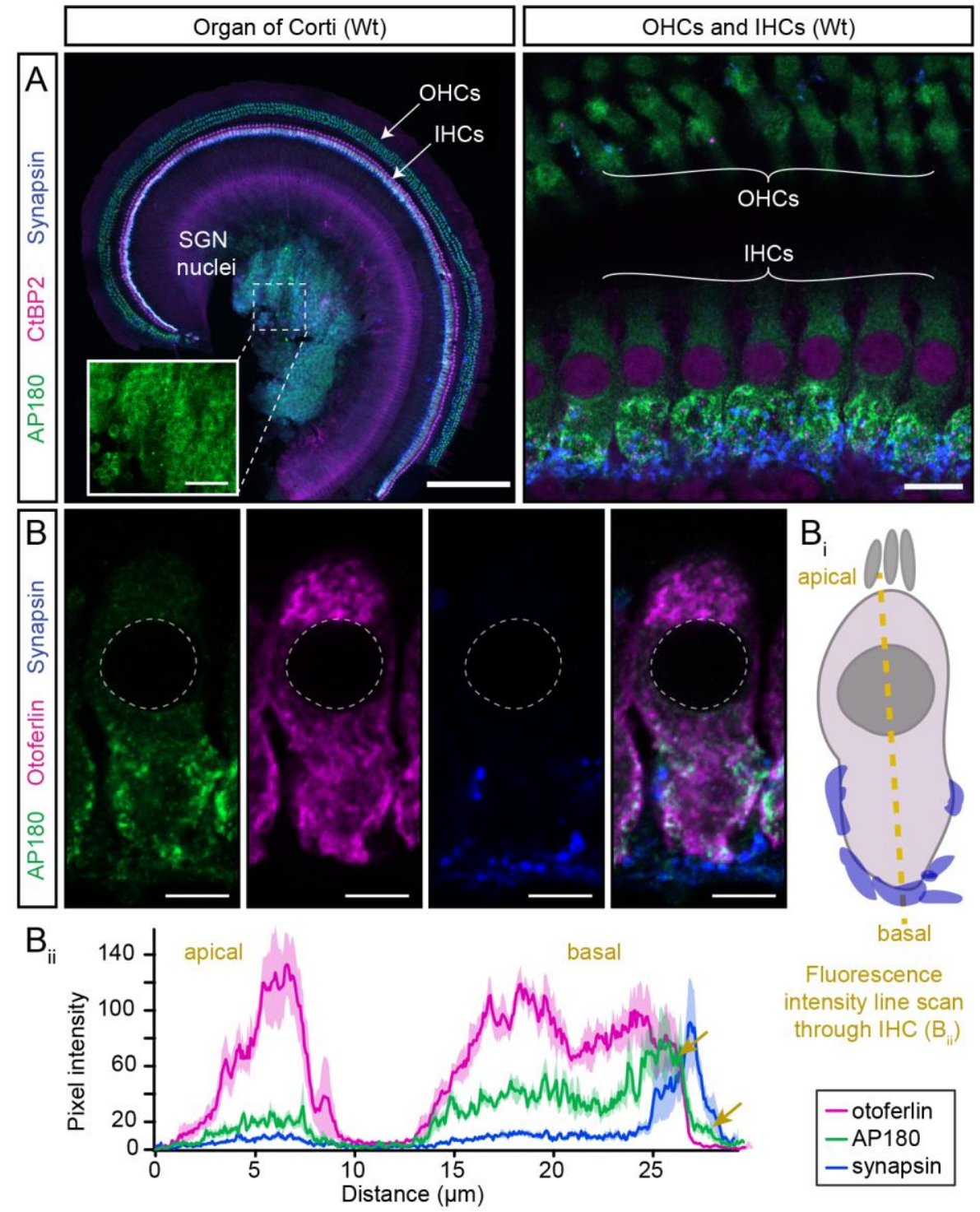

Fig. 3-1: AP180 is present in the murine organ of Corti. (A) Maximum intensity z-projection of confocal sections of an apical cochlear turn (left) and higher magnification of IHCs and OHCs (right) from a P15 Wt animal immunolabeled for AP180, RIBEYE/CtBP2 (staining ribbons and nuclei) and synapsin 1/2 (found in the nerve terminals of lateral olivocochlear efferents but not in IHCs). Scale bars: $200 \mu \mathrm{m}$ (left); $50 \mu \mathrm{m}$ (inset left); $10 \mu \mathrm{m}$ (right). The inset in (A) represents a magnification into the spiral ganglion area and shows AP180 immunofluorescence in the somata of spiral ganglion neurons. (B) Single confocal sections from a synaptic pole of an IHC (Wt, P15) using markers for AP180, otoferlin and synapsin 1/2. Scale bars: $5 \mu \mathrm{m}$. ( $\left.\mathbf{B}_{\mathbf{i}}, \mathbf{B}_{\mathbf{i i}}\right)$ Average fluorescence intensity line profiles through the longitudinal axis of seven IHCs from apical to basal. AP180 fluorescence is strongest in proximity to the IHC basal membrane, as indicated by yellow arrows (thereby overlapping with the otoferlin signal), but can also be detected in efferent nerve terminals (overlapping with the synapsin $1 / 2$ signal) and potentially in the postsynaptic boutons of spiral ganglion neurons. 
Higher magnifications of maximum intensity z-projections (Fig. 3-1A right panel) revealed that AP180 is most prevalent in the basal half of IHCS where the ribbon synapses are located ('synaptic pole'). Single xy-sections through the synaptic pole of immunolabeled IHCs show an intracellular as well as plasma membrane distribution of AP180. Line profiles through the longitudinal axis of IHCS (Fig. 3-1B $\mathrm{Bii}_{\mathrm{i}}$ ) revealed that the highest fluorescence intensity levels were at the IHC plasma membrane, followed by the IHC lumen and efferent presynaptic terminals.

The distribution of AP180 immunofluorescence was altered upon high- $\mathrm{K}^{+}$depolarization (65 mM for 1 min, Fig. 3-2B). Compared to the resting condition, the average intensity of AP180 fluorescence was significantly reduced in a distance of $2 \mu \mathrm{m}$ from the plasma membrane (Fig. 3-2C $\mathrm{ii} ; \mathrm{p}=0.0003$, unpaired student's t-test) after high- $\mathrm{K}^{+}$stimulation, whereas the staining near the membrane was preserved (Fig. 3-2C $C_{i} ; p=0.5468$, unpaired student's t-test). We further observed that high-intensity AP180 immunofluorescence clusters were al ready present in the proximity of the plasma membrane at the synaptic pole in the resting condition, but appeared even more intense after stimulation (Fig. $\left.3-2 \mathrm{~B}_{\mathrm{i}}\right)$. While the size of the clusters did not differ after $1 \mathrm{~min}$ of high- $\mathrm{K}^{+}$stimulation compared to the resting condition (Fig. 3-2D; $p=0.105, M$ ann-W hitney $U$ test), we found a higher mean intensity of AP180 immunofluorescence clusters after stimulation (Fig. 3-2D;; $p<0.0001$, M ann-W hitney U test). These data indicate a stimulation-induced recruitment of AP180 to the plasma membrane, pointing towards a relevance of AP180 for IHC synaptic transmission.

\section{AP180 AbSENCE Does not Alter OtOFERLIN LEVELS BUt VGLUT3 FLUORESCENCE}

In IHCs of mice with a knockout of the AP-2 subunit AP-2 $\mu$ (also known as AP2M 1), otoferlin levels are known to be strongly reduced, with the reduction being less pronounced at the plasma membrane, suggesting impai red sorting of otoferlin (Jung et al., 2015b). Therefore, wetested by semiquantitative analysis of otoferlin immunofluorescence if deletion of AP180 likewise changes otoferlin levels and/or distribution (Fig. 3-3A and $A_{i}$ ). Yet, neither otoferlin distribution, nor total fluorescence intensity, were altered in IHCs of AP180-KO mice compared to Wt ( $p=0.17$, unpaired student's ttest). However, we noted that the immunofluorescence intensity of the vesicular glutamate transporter of IHCs, V glut3 (also known as SLC17A 8), was reduced by $17.1 \pm 3.2 \%$ throughout the IHC in AP180-KO mice compared to Wt (Fig. 3-3B and $B_{i} ; p<0.0001$, unpaired student's t-test). Notably, co-staining for RIBEYE/CtBP2 and Homer1 (a marker for the postsynaptic density; Fig. 3$3 C$ ) indicated an unaltered ribbon anchorage to the $A Z$ as well as a normal number of ribbon 
synapses (Fig. 3-3C ${ }_{i}$; ribbon synapses per IH C for W t: $14.5 \pm 0.4$, for AP 180-K O: $14.5 \pm 0.5$ synapses per IHC; $p=0.96$, unpaired student's t-test) in AP180-KO mice.
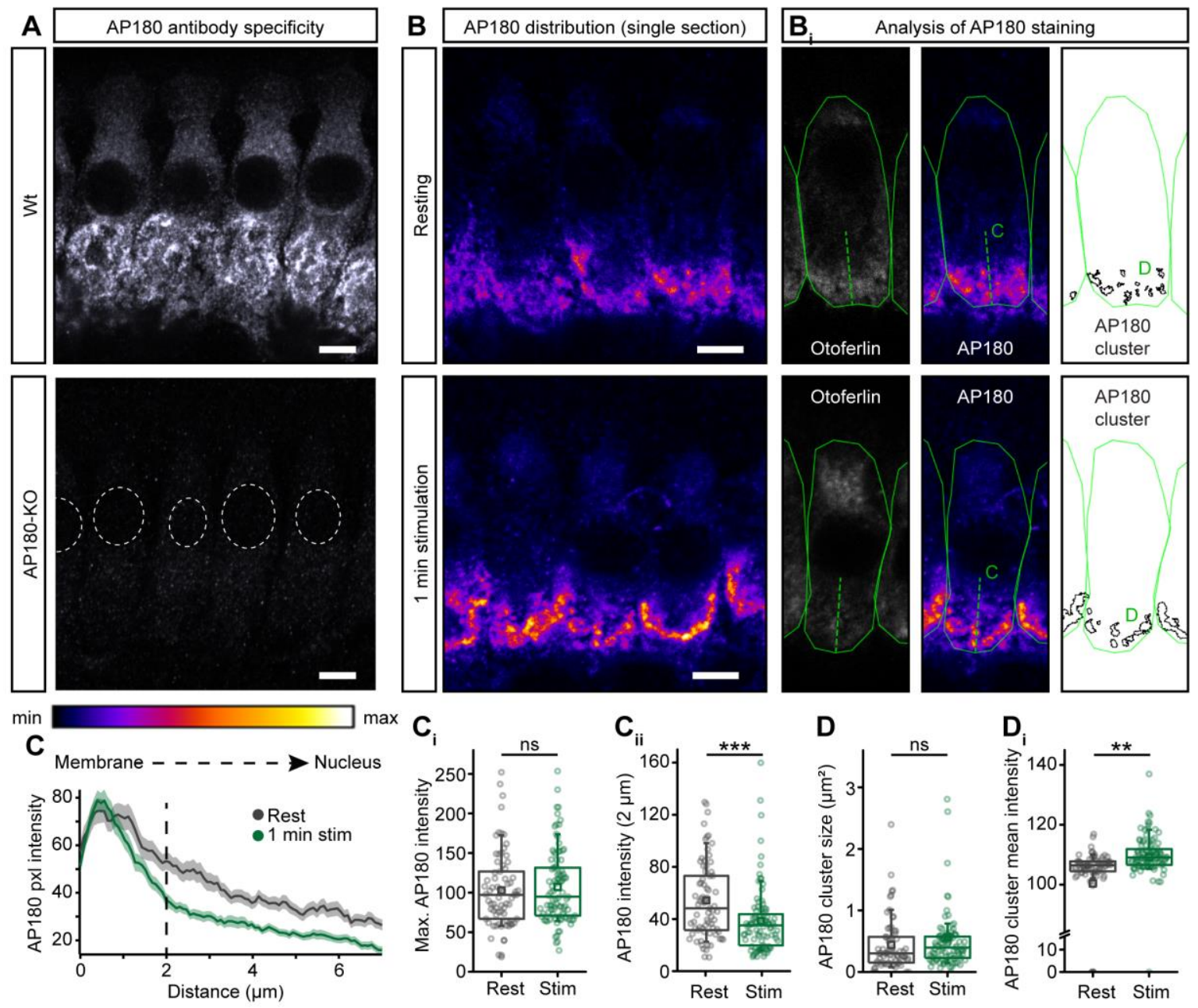

Fig. 3-2: Subcellular distribution of AP180 changes after stimulation. (A) AP180 immunofluorescence in maximum intensity z-projections of confocal sections of apical cochlear turns from Wt and AP180-KO mice (both P15). Scale bar: $5 \mu \mathrm{m}$. (B) Exemplary single confocal sections from synaptic pole of IHCs from Wt P15 mice after 1 min high $\mathrm{K}^{+}$stimulation compared to IHCs after 1 min in low $\mathrm{K}^{+}$control solution. Scale bars: $5 \mu \mathrm{m}$. ( $\left.\mathbf{B}_{\mathbf{i}}\right)$ Visualization of analysis approaches to determine AP180 immunofluorescence. Left: cell borders and a vertical line from the basal IHC pole towards the cytosol were defined according to otoferlin costaining. Middle: in the AP180 channel, immunofluorescence intensities along the line scans were measured. Right: High-intensity AP180 immunofluorescence clusters were defined using an intensity threshold. (C) Average AP180 intensity line scans from confocal sections measured from the basal IHC membrane towards the nucleus reveal unchanged max. intensities in membrane proximity $\left(\mathbf{C}_{\mathbf{i}}\right)$ and reduced AP180 intracellular levels in $2 \mu \mathrm{m}$ distance from the membrane $\left(\boldsymbol{C}_{\mathrm{ii}}\right)$ after stimulation $(\mathrm{n}=93 \mathrm{IHCS}$ from $N=3$ mice) compared to resting condition ( $n=73 \mathrm{IHCs}$ from $N=3$ mice). (D) The average size of highintensity AP180 clusters did not differ between resting and stimulated condition, whereas stimulation led to an increase in average cluster fluorescence intensity $\left(\mathbf{D}_{\mathbf{i}}\right) . * * p<0.01 ; * * *<0.001$. 
In summary, deletion of AP-180 did not alter otoferlin levels, synapse number or ribbon anchorage, but it reduced V glut3 protein levels, potentially via diminishing SV numbers.

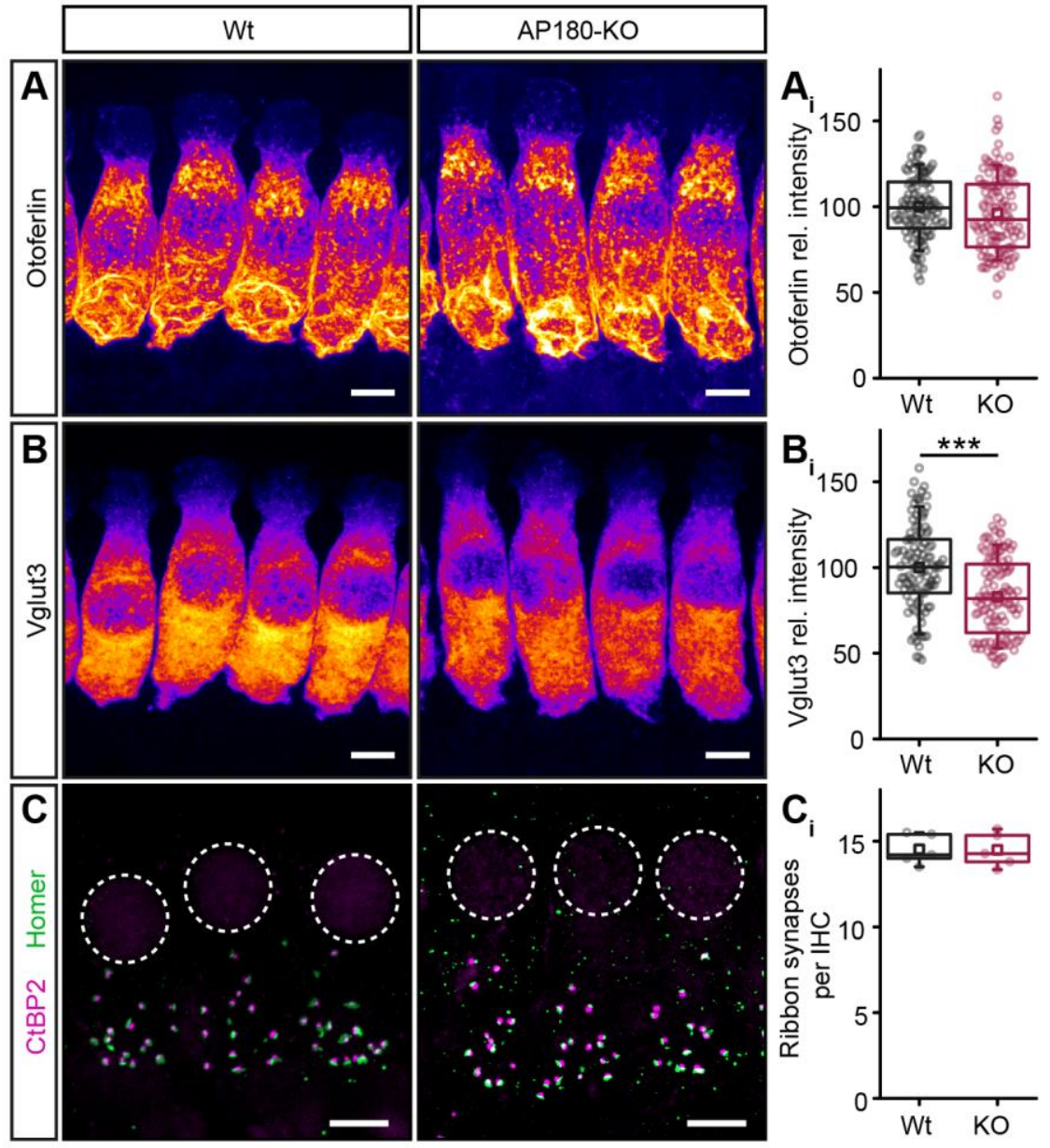

Fig. 3-3: Reduced Vglut3 levels, but unaltered otoferlin levels in AP180-deficient IHCs. (A) Maximum intensity z-projections of otoferlin-stained apical cochlear turns from AP180-KO and Wt littermate controls. $\left(\mathbf{A}_{i}\right)$ Unaltered otoferlin levels in AP180-KO $(n=114 \mathrm{IHCs}$ from $\mathrm{N}=4$ mice) compared to Wt ( $n=107 \mathrm{IHCS}$ from $N=4$ mice). (B) Maximum intensity z-projections of confocal sections of Wt and AP180-KO IHCS stained for Vglut3. $\left(\mathbf{B}_{\mathbf{i}}\right.$ ) Intracellular Vglut3 levels were reduced by $\sim 17 \%$ in AP180-KO IHCs ( $n=114 \mathrm{IHCS}$ from 4 mice) compared to Wt ( $n=107 \mathrm{IHCs}$ from $\mathrm{N}=4$ mice). (C) Exemplary confocal sections of RIBEYE/CtBP2 (magenta) labeling the synaptic ribbon and Homer1 (green) staining labeling the postsynaptic density. Dashed circles highlight the nuclei. $\left(\mathbf{C}_{\mathbf{i}}\right)$ Equal numbers of ribbon synapses in Wt 6 images from 3 animals, approx. 10 cells per image) and in AP180-KO (6 images from 3 animals, approx. 10 cells per image). ${ }^{* * *} p<0.001$. Scale bars: $5 \mu \mathrm{m}$.

\section{Numbers of SVs And Clathrin-COATEd StruCtuRES ARe ReduCEd In IHCS OF AP180-KO MICE}

N ext, we performed high-pressure freezing followed by freeze substitution and electron tomography of ribbon synapses from P15-P16 mice (shortly after the onset of hearing) to assess the synaptic 
ultrastructure. Exemplary virtual sections (Fig. 3-4A), obtained from semi-thin sections via tomogram generation, as well as 3D models of reconstructed ribbon synapses from Wt and AP180KO IHCs (Fig. 3-4B) revealed reduced numbers of SV s not only in direct vicinity to the ribbon, but also in the cytoplasm. We investigated the following three morphological pools of SV s, as defined previously (Kroll et al., 2019) and indicated in the schematic drawing (Fig. 3-4D): (i) ribbonassociated SV s (RA-SV s) in a maximum distance of $80 \mathrm{~nm}$ to the ribbon, (ii) membrane-proximal SV s (M P-SV s) that are within a $50 \mathrm{~nm}$ distance from the plasma membrane and $100 \mathrm{~nm}$ laterally from the presynaptic density, and (iii) cytosolic SV s (operationally defined as all uncoated vesicles with a maximum outer diameter of $70 \mathrm{~nm}$ and a maximum distance of $500 \mathrm{~nm}$ to the ribbon, excluding the aforementioned SV pools). In all three pools, numbers of SV s were reduced in IHCs of A P180-KO mice compared to $\mathrm{Wt}$. The strongest reduction by $\sim 60 \%$ was observed for the pool of cytosolic SV s (Fig. 3-4I; $p<0.0001$, unpaired student's t-test). Numbers of RA-SV s (Fig. 3-4E; $p=$ 0.0062, unpaired student's t-test) and of M P-SV s (Fig. 3-4F; $p=0.0328$, unpaired student's t-test) were reduced by approximately $24 \%$ and $22 \%$, respectively. The reduction of SV s, found in electron tomography, exceeded the expectation based on V glut3 immunofluorescence (reduction by $\sim 17 \%$ ). The outer diameter of the cytosolic SV s, on the other hand, was increased on average (Fig. 3-4); $p<$ 0.0001, unpaired student's t-test) and more variable in IHCs of AP180-KO mice compared to Wt ( $p$ $<0.0001$, Kolmogorov-Smirnov test), which may account for the modest reduction of V glut3 fluorescence intensity. The observed increase of theSV diameter is consistent with results of previous studies on mice and invertebrates missing A P180 or its homolog U NC-11 (Koo et al., 2015; N onet et al., 1999; V anlandingham et al., 2014; Zhang et al., 1998). Interestingly, the average distance of M PSV s to the AZ plasma membrane was slightly increased in IH C s from AP180-KO mice compared to Wt (Fig. 3-4G; $p=0.0288, M$ ann-Whitney $U$ test).

We further quantified numbers of clathrin-coated structures in proximity to the ribbon, including coated vesicles and coated pits budding from theplasma membrane or from ELVs, examples of which can be seen in Fig. 3-4C. The numbers of coated structures in total (Fig. 3-4H; $p=0.0002$, unpaired student's t-test), and of coated vesicles in particular ( $p<0.0001, M$ ann-W hitney $U$ test, please also see Appendix Table S16 for further information on statistical analyses), were strongly reduced in AP180-KO mice compared to Wt. This suggests an involvement of AP180 in the early steps of clathrin-mediated membrane retrieval. While numbers of ELV s were not significantly increased in tomograms of AP180-KO mice (Fig. 3-4K; $p=0.7645$, unpaired student's t-test), we found a strong 
increase in their average volume (Fig. 3-4L; $p=0.0043$, $M$ ann-W hitney $U$ test). Therefore, it is likely that the reduced numbers and increased size of SVs are a consequence of impaired clathrin- and AP180-dependent SV reformation from ELVs.

Deletion of AP180 Impairs CME ANd CLATHRIN-DEPENDENT SV ReFORMATION IN IHCS

Given the role of AP180 in CME and clathrin-dependent SV reformation at conventional synapses (Koo et al., 2015; Zhang et al., 1998), we investigated the morphology of I H Cs from AP180-KO mice after stimulation. For that, we stimulated freshly dissected P15-P16 organs of Corti for 5 min using high- $\mathrm{K}^{+}$followed by high-pressure freezing, freeze substitution and electron tomography (Fig. 3-5).
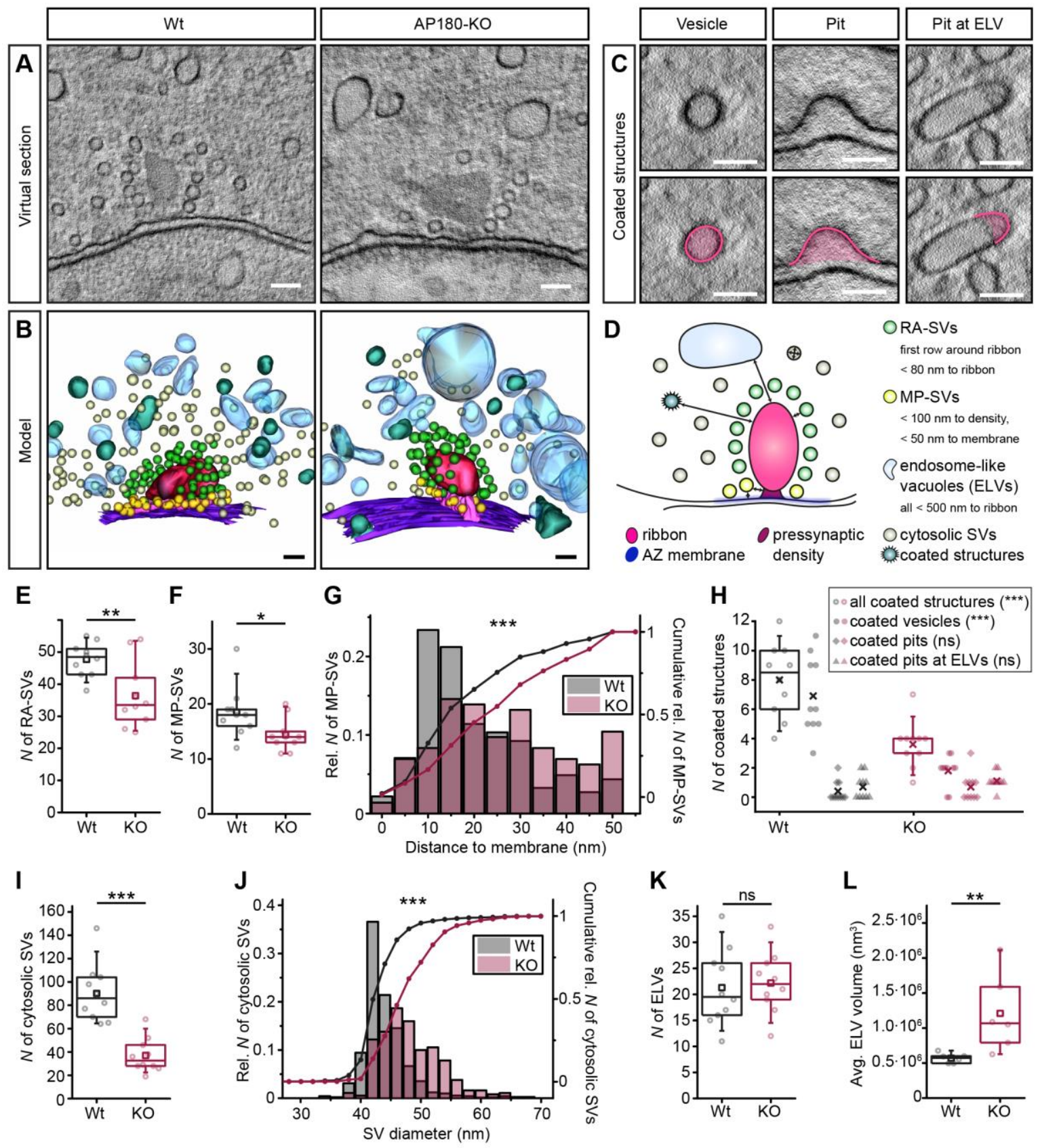
Fig. 3-4: Ultrastructural changes of IHC ribbon synapses in AP180-KO mice. (A) Representative virtual sections of tomograms from P15 Wt and AP180-KO ribbon synapses. Scale bars: $100 \mathrm{~nm}$. (B) Reconstructed models of the area around the ribbon from P15 Wt and AP180-KO IHCs. Scale bars: $200 \mathrm{~nm}$. (C) Exemplary coated structures observed at the IHC base. Scale bars: $100 \mathrm{~nm}$. (D) Schematic drawing illustrating parameters for the quantifications in (E-L); $n$ for all quantifications $=10$ tomograms from 2 different animals in each group. (E) Numbers (N) of RA-SVs were reduced by $\sim 24 \%$ in AP180-KO mice. (F) The number of MPSVs was likewise reduced, and (G) the distance of MP-SVs to the AZ plasma membrane was increased. (H) Numbers of coated structures were decreased with the number of coated vesicles being most strongly affected. (I) In the cytosol, numbers of small uncoated vesicles were drastically ( $>60 \%)$ reduced in absence of AP180. (J) The average diameter of cytosolic SVs was increased. Generally, SV diameters were more heterogeneous in absence of AP180. (K) Numbers of ELVs were unaltered, (L) while the average volume per ELV was strongly increased in AP180-KO mice. $* p<0.05$; $* *<0.01$; $* * p<0.001$; ns, not significant.

Similar to what is found in resting conditions, we observed a lower total number of coated structures in IHCS of AP180-KO mice (Fig. 3-5I, p = 0.002, M ann-W hitney $U$ test). We detected several ELV s with multiple budding CCPs in proximity to ribbon synapses in $\mathrm{Wt}$, indicative of active SV reformation following bulk endocytosis (an example is shown in the virtual section and in the 3D model in Fig. 3-5C). However, in AP180-KO mice, we did not observe ELV s with more than one or two budding CCPs. M oreover, the number of ELV s with one or more budding CCPs was reduced in AP180-KO IHCs compared to Wt (Fig. 3-5I; $p=0.006$, M ann-W hitney $U$ test, also see Appendix Table S17). As seen at rest, the total number of ELV s was unaltered in AP180-K O IHCs compared to Wt ( $p=0.113$, student's t-test, Appendix Table S17) whereas the average ELV volume was again increased (Fig. 3-5H; $p=0.0355$, M ann-W hitney $U$ test).

The number of cytosolic SV s was decreased by more than 40\% in AP180-KO IHCs (Fig. 3-5F; $p=$ 0.0026, Mann-Whitney $U$ test), and, hence, did not go beyond the reduction found at resting conditions. Interestingly, we neither observed a difference in the number of RA-SV s (Fig. 3-5D; $p=$ 0.445, $M$ ann-W hitney $U$ test) nor in the number of M P-SV s (Fig. 3-5E; $p=0.721$, M ann-W hitney $U$ test) between Wt and AP180-KO mice after stimulation. These data indicate that AP180- and clathrin-dependent reformation of SV s is required for maintaining high numbers of cytosolic SV s in the synaptic pole of the IHC. Still, even in absence of A P180, endocytic SV recycling was sufficient to provide enough SV s for refilling the pools of RA-SV s and M P-SV s. 


\section{After Stimulation, SVs Remain in a Multi-tethered State at the AZ of AP180-KO IHCS}

We examined the M P-SV pool further by electron tomography (Fig. 3-5A and 3-5B). As mentioned above (Fig. 3-4G), the distance of M P-SV s to the AZ membrane was increased in AP180-KO IHCS under resting conditions. After stimulation, we did not observe significant differences in the average distance to the AZ membrane for M P-SV s between Wt and AP180-KO IHCs (A ppendix Table S17; $p=0.451$, unpaired student's t-test). Since SV tethering to the AZ membrane has been suggested to influence the distance between M P-SV s and AZ membrane (e.g. Chakrabarti et al., 2018; FernándezBusnadiego et al., 2013), we quantified the numbers of tethers to the AZ membrane under resting conditions and after stimulation. For that purpose, we defined the following SV groups: (i) SV s without such tethers, (ii) SV s with a single tether, (iii) SV s with multiple tethers, and (iv) docked SV s, where the distance between SV outer membrane and AZ membrane was 0-2 nm in electron tomography virtual sections, as previously described for IH Cs and neurons (Chakrabarti et al., 2018; Imig et al., 2014).

In resting conditions, we did not observe any significant differences in the tethering of M P-SV s in AP180-KO IHCs compared to the Wt situation (Fig. 3-5G, one-way ANOVA followed by Tukey's post-hoc test; for individual p-values see A ppendix Table S18). In line with a previous study of IH Cs, high- $\mathrm{K}^{+}$stimulation resulted in relatively more tethered SV s (Chakrabarti et al., 2018). This was the case in both, Wt and AP180-KO IHCs (Fig. 3-5G; for the fraction of untethered SV s, resting Wt vs. stimulated Wt $p=0.001$, resting Wt vs. stimulated AP180-KO $p=0.001$, one-way A N OVA followed by Tukey's post-hoc test). However, we noticed a shift towards an increase in multi-tethered SV s in A P180-KO IHCs after stimulation, whereas most SV s in stimulated Wt IHCs remained connected via a single tether ( $p=0.008$ for the fraction of multi-tethered MP-SV s in stimulated Wt vs. stimulated AP180-KO IHCS). Furthermore, we observed significantly more docked SVs in stimulated AP180-KO IHCs compared to resting conditions (Wt resting condition vs. AP180-KO stimulated condition: $p=0.004$, one-way ANOVA followed by Tukey's post-hoc test; AP180-KO rest. vs. AP180-KO stim: $p=0.002$, one-way ANOVA followed by Tukey's post-hoc test), as well as compared to stimulated Wt ( $p=0.037$, one-way ANOVA followed by Tukey's post-hoc test). Together, these observations indicate that M P-SV s in IH Cs of A P180-KO mice remain in the multitethered or docked state prior to fusion, which has previously been designated as a morphological correlate of impaired release site clearance in IHCs (Chakrabarti et al., 2018). 

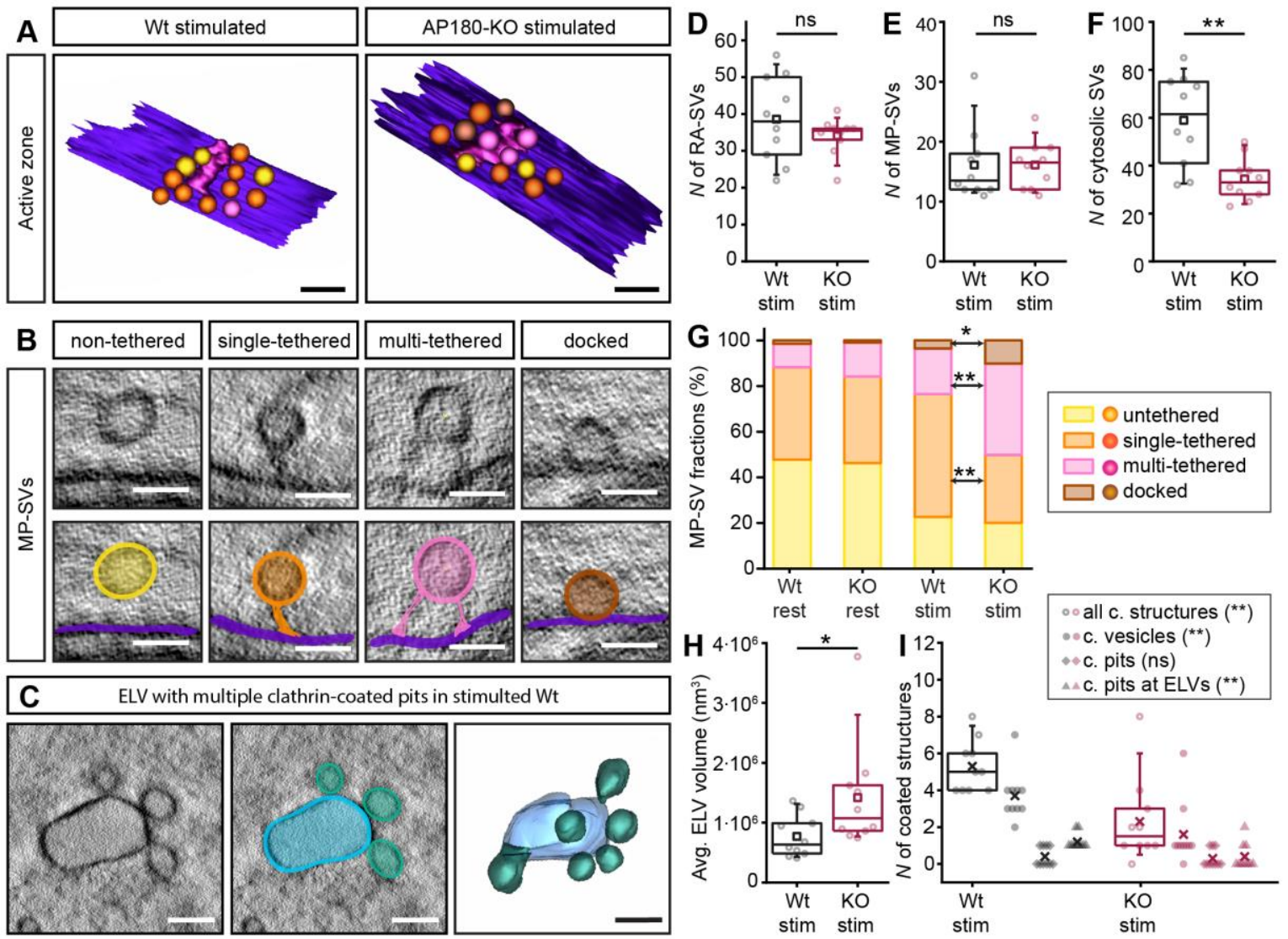

Fig. 3-5: SV-tethering to the AZ and SV reformation are altered in stimulated IHCs from AP180-KO

mice. (A) Reconstructed models of the AZ from P15 Wt and AP180-KO IHCs after 5 min $\mathrm{K}^{+}$stimulation showing the presynaptic density and MP-SVs. Colors of different classes of MP-SVs are as highlighted in (B). Scale bars: $200 \mathrm{~nm}$. (B) Exemplary virtual sections of MP-SVs in untethered, tethered or docked state. Scale bars: $50 \mathrm{~nm}$. (C) Virtual section and 3D model of an ELV containing multiple CCPs, as exclusively observed in Wt. Scale bar: $100 \mathrm{~nm}$. (D) Numbers (N) of RA-SVs were unaltered in P15 AP180-KO mice compared to Wt ( $\mathrm{n}$ for D-I = 10 tomograms from 2 different animals per group). (E) The number of MP-SVs was likewise unaltered in absence of AP180 compared to Wt, whereas numbers of small uncoated vesicles were strongly reduced $(\mathbf{F})$. (G) Examining numbers of tethers, a significantly higher proportion of multi-tethered and docked MP-SVs have been observed combined with a relatively lower number of single-tethered SVs in absence of AP180 after stimulation compared to stimulated Wt as well as compared to resting condition in Wt and AP180-KO. (H) The volume of ELVs was increased in the AP180-KO mice. (I) Numbers of coated structures were decreased with numbers of coated vesicles and coated pits at ELVs being most strongly affected. $* p<0.05 ; * *<0.01 ; n s$, not significant. 


\subsubsection{DISCUSSION}

In the present study, we addressed a role of the endocytic adaptor protein AP180 in synaptic sound encoding in the cochlea. There, IHC ribbon synapses must sustain high rates of SV releasefor reliable transmission of auditory information. When investigating IHC ribbon synapses of AP180-KO mice by performing a combination of immunohistochemistry and electron tomography, we found evidence for a dual function of AP180. Firstly, AP180 is required for clathrin-dependent SV reformation from ELV s and aids in the early steps of classical CME. Secondly, AP180 plays a role in efficient replenishment of SV s to the release site, likely via promoting the clearance of the release site from proteins and lipids added by preceding SV fusion events.

AP180 IS REQUIRED FOR CLATHRIN-DEPENDENT ENDOCYTOSIS AND SV REFORMATION FolLOWING BuLK RetRIEVAL IN IHCS

The reduction of clathrin-coated SV s in resting and stimulated AP180-KO IHCs support a role of AP180 in CME. Beyond that, we observed less budding of CCPs from enlarged ELV s in AP180-KO IHCs after high-K+ $\mathrm{K}^{+}$stimulation, suggesting that SV reformation from ELV s following bulk retrieval operates in an AP180-dependent manner. Clathrin-dependent SV reformation from ELV s was also impaired in A P-2 KO IHCs (Jung et al., 2015b). Therefore, like in hippocampal neurons (Kononenko et al., 2014; K oo et al., 2015), both adaptor proteins seem to be required for the recruitment of clathrin triskelia not only to the plasma membrane, but also to ELVs. Notably, SV numbers were more robustly reduced in AP180-deficient IHCS $(\sim 60 \%)$ than in AP180-deficient inhibitory CNS neurons ( 33\%; note that no SV reduction was found in excitatory neurons, Koo et al., 2015). This underlines the importance of clathrin- und A P180-dependent SV reformation from endocytic intermediates for the restoration of SV pools particularly in cells with a high SV turnover rate like the IHCS.

Nonetheless, CM E still occurred. M oreover, prolonged stimulation by high- $\mathrm{K}^{+}$left the RA- and M PSV s pools unchanged and did not further reduce the number of cytosolic SV s in AP180-KO IHCS. Therefore, AP180-independent forms of membrane retrieval must exist in IHCs, and other adaptor proteins liketheA P180 homolog CA LM , AP-2 or A P-3 may contribute to the recruitment of clathrin. The precise interplay of adapter proteins and membrane retrieval mechanisms in IHCS requires further investigation. Also, future studies might address the question whether accumulation of exocytosed proteins and lipids at the plasma membrane, or increased membrane tension, shift membrane retrieval towards clathrin-independent forms ( $M$ aritzen and H aucke, 2018). 


\section{A Role of AP180 in Release Site Clearance at IHC Active Zones}

N otwithstanding the SV reformation deficit, the number of M P-SV s at the AZ was not significantly reduced in AP180 KO IHCs during strong stimulation. Still, the measured rate of SV replenishment during trains of brief depolarizations was reduced in AP180-KO IHCs, while $\mathrm{Ca}^{2+}$ triggered fusion of the RRP of SV s seemed intact (data not shown in this chapter). These findings suggest that slowed SV replenishment of the release sites was primarily due to impaired AZ clearance rather than to insufficient SV resupply to the AZ. Effective release site clearance has previously been discussed as a determinant of sustained exocytosis in hair cells (Chakrabarti et al., 2018; Cho et al., 2011; Jung et al., 2015b; Kroll et al., 2019; Pangršič et al., 2010). Further support for this hypothesis comes from the observation of an increased number of multi-tethered and docked SV s in the AP180-KO IHCS. Similarly, in IHCs of the otoferlin mouse mutant pachanga, which has been shown to be profoundly deaf with a reduced rate of SV replenishment to the RRP (Pangršič et al., 2010), the number of multitethered SV s was significantly higher than in W t after stimulation (Chakrabarti et al., 2018). M ultitethered SV s in neurons were also shown to be closer to the AZ membrane compared to singletethered or untethered SV S and probably display a prerequisite for SV docking and priming (Fernández-Busnadiego et al., 2013). As SV fusion was intact in AP180 KO IHCS, we take the higher prevalence of multi-tethered SV s as a potential morphological correlate of unproductive release sites due to impaired clearance.

In a previous study, absence of RIM - binding protein 2 (RIM -BP2) likewise resulted in an increased distance of MP-SV s to the AZ-membrane in IHCs at resting conditions, which has been attributed to disturbances in the composition of the cytomatrix of the AZ (Krinner et al., 2017). Changes in the cytomatrix or in the AZ plasma membrane itself may also serve as an explanation for the increased distance of MP-SVs in absence of AP180. More precisely, the absence of AP180 could lead to an accumulation of proteins or lipids at the membrane of the release sites, which possibly causes an energetically unfavorable environment, ultimately slowing down exocytic processes after prolonged stimulation.

AP180 has previously been identified as a key sorting factor for VAMP2. Consequently, AP180 deficiency causes an accumulation of VAM P2 at the plasma membrane of neurons (Koo et al., 2015). Even though IHCs seem to operate without VAM P2 and other neuronal SNARE proteins (N ouvian et al., 2011), A P180 could be required for the sorting of other members of the VA M P family that were 
previously identified as additional interaction partners of A P180/CALM (M aritzen et al., 2012; M iller et al., 2011; Sahlender et al., 2013). Alternatively, an as-yet unknown exocytic IHC protein may interact with AP180 and accumulate at the plasma membrane in the absence of AP180. I mportantly, unaltered otoferlin levels suggest that otoferlin sorting does not depend on AP180 and consequently allow us to attribute the IHC phenotype to AP180 deficiency itself rather than to the concomitant loss of otoferlin. 


\section{DISCUSSION}


Discussion | Manuscript 


\subsection{NEW INSIGHTS INTO SV RECYCLING IN IHCS}

Over the last two decades, IHCs have not only been investigated to identify causes for hearing impairments and to examine deafness genes. The extraordinarily high rates of SV turnover and the unique features of ribbon synapses made IHCs also become a model system for synaptic transmission. Previous studies have shown that exocytosis in IHCs is specialized, meaning that SV release seems to operate without classical SN ARE proteins (N ouvian et al., 2011) and neuronal $\mathrm{Ca}^{2+}$ sensors (Beurg et al., 2010; Reisinger et al., 2011; Safieddine and W enthold, 1999). Instead, otoferlin and other yet to be identified proteins mediate exocytosis (Pangršič et al., 2010; Roux et al., 2006). M oreover, otoferlin was shown to play a role in the coupling of exo- and endocytosis, in release site clearance, and potentially also directly in endocytosis (Chakrabarti et al., 2018; Duncker et al., 2013; Jung et al., 2015b; Pangršič et al., 2010). SV recycling may therefore likewise be adapted to the very high demands of IHCs. A part from roles of dynamin and clathrin in CME and from the importance of AP-2 in SV reformation following bulk-like membrane retrieval, little has been known about the peculiarities of IHC endocytosis. Therefore, I focused my studies on two proteins that were characterized in depth as key players in neuronal endocytosis: W hile AP180 is involved in the early steps of CM E and clathrin-dependent SV reformation (Lindner and U ngewickell, 1992; M orris et al., 1993), endophilin was shown to beessential for fission and uncoating (M ilosevic et al., 2011; Ringstad et al., 1997; Schuske et al., 2003; Verstreken et al., 2002). M oreover, roles of endophilin in clathrinindependent forms of endocytosis have been described not only in neurons (W atanabe et al., 2018) but also in non-neuronal cells (Boucrot et al., 2015; Renard et al., 2015). Studying the functions of both proteins, I could not only assess their putative roles in IHCs endocytosis. I further gained information on the prevalence of different endocytic mechanisms in IHCs, which I will address in the first part of my discussion. In addition, I could show together with the co-authors of the here presented studies that both proteins, AP180 and endophilin, are involved in processes beyond classical endocytosis, that partially differ from their functions in neurons. In the second part of my discussion, I will elaborate the putative roles of AP180 and endophilin in the coupling of exo- and endocytosis, in release site clearance and in SV resupply in more detail. 


\subsubsection{ENDOPHILIN AND AP180 ARE INVOLVED IN CLATHRIN-MEDIATED ENDOCYTOSIS IN IHCS}

Previous studies have shown that the slow mode of endocytosis observed via electrophysiological recordings in IH Cs is clathrin- and dynamin-dependent (M oser and Beutner, 2000; N eef et al., 2014). In the here presented two studies, I could show together with my collaborators, that the formation of endocytic intermediates is also affected by the absence of endophilin or AP180. In the first part, we examined a number of mutants missing different combinations of the three endophilin-A genes. Electron microscopy of conventionally embedded organs of Corti revealed higher numbers of typical CM E intermediates: Particularly in DKOs of endophilin A 1 and A2 (1/2-DKOs) and of endophilin A 1 and A3 (1/3-DKOs), I observed accumulations of CCV s and coated vacuoles (larger organelles with a diameter of more than $70 \mathrm{~nm}$ and entirely covered by a clathrin coat) as well as CCPs not only at the plasma membrane but also at ELVs. Furthermore, I found a greater area covered by ELV s in both DKOs and a reduced number of cytosolic vesicles in 1/2-DKOs. The number of RA-SV s and M P-SV s was lower in both DKO s with the RA-SV s of the distal half of the ribbon being morestrongly affected than those of the proximal half. The findings regarding endocytic intermediates and SV numbers are in-line with previous studies, in which accumulations of CCV s and ELVs, paired with reduced numbers of SVs, have been described for neurons of endophilin 1/2-DKOs and TKOs (M ilosevic et al., 2011). Despite the significant ultrastructural changes in the endophilin mutants, patch-clamp recordings of endocytosis revealed an only mildly decreased rate of the linear component that likely reflects CM E. Furthermore, amplitude and time constant of the exponential phase, likely reflecting bulk-like retrieval, were slightly affected.

In AP180-KO mice, electron tomography following HPF and freeze substitution revealed a significantly lower number of coated structures in proximity to the ribbon. Particularly the number of CCVs was reduced whereas we could not detect strong effects on the number of CCPs. Even though the number of ELVs was unchanged in AP180-KOs, we detected a significantly increased average ELV volume. Furthermore, the number of vesicles was drastically reduced in the cytosol (by roughly $60 \%$ ) and slightly reduced around the ribbon (by approx. 20\%). These data are partially in agreement with previous studies of neurons from AP180-KO mice (Koo et al., 2015): here, excitatory neurons exhibited unchanged SV numbers while inhibitory neurons showed a slight reduction. H owever, numbers of coated structures were likewise decreased whereas the total ELV volume per 
nerve terminal was elevated. $\Delta \mathrm{C}_{\mathrm{m}}$ recordings from mouse IHCs following $20 \mathrm{~ms}$ or $200 \mathrm{~ms}$ long stimulation indicated that the exponential phase of endocytosis is not at all, and the linear phase of endocytosis is only slightly affected by AP180 absence (experiments performed by Özge Demet Özçete, Institute for A uditory N euroscience, Göttingen).

\subsubsection{AP180 HAS A ROLE IN CLATHRIN RECRUITMENT IN IHCS}

It is widely accepted that the adaptor protein-mediated recruitment of clathrin represents the early phase of CME (Haucke and Kozlov, 2018; Saheki and Camilli, 2012). Through binding sites for clathrin, $\mathrm{PI}(4,5) \mathrm{P}_{2}$ and for each other, AP180 and AP-2 are supposed to bring clathrin triskelia to the nucleation sites, at which CCPs are formed in neurons (Ford et al., 2001; Hao et al., 1999). The depletion of AP-2 was shown to cause reductions in numbers of coated structures in neurons (M CM ahon and Boucrot, 2011) as well as in IHCs (Jung et al., 2015b), comparable to what was observed in neurons lacking AP180 (Koo et al., 2015). I could show that absence of AP180 in IHCs results in a similar phenotype. Given that AP-2 (Jung et al., 2015b and recent observations of Dr. Christian V ogl, Institutefor A uditory N euroscience, Göttingen, seeA ppendix Fig. S1) and AP180 (see Fig. 3-1) are highly prevalent in the basal part of IHCs, I suggest that these proteins together are involved in the recruitment of clathrin also in IHCs. Of note, AP-2 and AP180 seem to benefit from the presence of the respective other protein, meaning that they do not only work in similar directions but probably boost each other ( $\mathrm{H}$ ao et al., 1999). Thus, AP-2 or A P180 alone may be less efficient, resulting in lower numbers of CCV sin IHCs as well as in neurons. In neurons from A P180-KO mice, even the concomitantly seen upregulation of the ubiquitously expressed AP180-homolog CALM was not sufficient to restore clathrin-dependent processes (K oo et al., 2015). In contrast to A P180, which harbors more than ten clathrin binding sites within the $\mathrm{C}$-terminal unstructured domain, only one to two clathrin binding motifs have been identified in CALM (see Fig. 1-5), possibly resulting in a lower clathrin binding affinity of CALM (Meyerholz et al., 2005; Morgan et al., 2000; M oshkanbaryans et al., 2014; Zhuo et al., 2010). Even though the expression of CALM was not part of my examinations, it is unlikely that an upregulation of this protein could sufficiently overtake all functions of AP180 in IHCS.

Prior or in parallel to the recruitment of clathrin triskelia, CALM is also involved in membrane bending (Haucke and Kozlov, 2018; Miller et al., 2015). In HeLa cells with depleted CALM expression, CCPs were flattened and CCV diameters were enlarged (M eyerholz et al., 2005; M iller et 
al., 2015). In vitro studies revealed that the ANTH domain, which is much more highly conserved between CALM and AP180 than the unstructured C-terminus, is crucial for membrane bending (M iller et al., 2015). An additional role of A P180 in membrane bending would explain the stronger heterogeneity of SV diameters and the overall increased size of SV s in absence of AP180 not only in neurons (Koo et al., 2015) but also at IHC ribbon synapses (see Fig. 3-4), as well as at Drosophila neuromuscular junctions missing the A P180 homolog LAP (Zhang et al., 1998). H owever, apart from CALM, other proteins like epsin (Chen et al., 1998; Ford et al., 2002) and BAR domain proteins (Gallop et al., 2006; Henne et al., 2010) were shown to act in membrane bending at different time points during pit formation. It is therefore unlikely that a potential role of AP180 in membrane bending and not the AP180-dependent recruitment of clathrin would be rate-limiting for the formation of CCPS.

\subsubsection{ENDOPHILIN IS REQUIRED FOR FISSION AND UNCOATING IN IHCS}

Whether the membrane bending abilities of endophilin, which have been characterized in depth in vitro (Gallop et al., 2006; M im et al., 2012), indeed play a role in vivo is still under debate (Saheki and Camilli, 2012). Studies in mouse neurons revealed a rate-limiting role of endophilin in the uncoating step (M ilosevic et al., 2011). Even though CCP numbers were not elevated in endophilin 1/2-DKOs and TKOS, endophilin has also been detected at CCPs in neurons before fission, where it is supposed to recruit dynamin (Ferguson et al., 2009; M ilosevic et al., 2011; Perera et al., 2006). Studies in

C. elegans and Drosophila likewise point towards key roles of endophilin in fission and uncoating (Schuske et al., 2003; V erstreken et al., 2002; Verstreken et al., 2003). H owever, another study has substantiated an important role of endophilin in membrane bending in Drosophila (Bai et al., 2010), suggesting that endophilin may be involved in different steps of CME depending on species and cell type.

In IHCs of endophilin DKOs, I did not only observe more coated structures in total, but more specifically accumulations of CCPs at the plasma membrane (see Fig. 2-7). I therefore propose that endophilin is not only required for uncoating, e.g. via recruitment of synaptojanin and/ or other uncoating factors, but has also a key role in membranefission in IHCs. Whether dynamin, which has already been shown to be involved in IHC endocytosis (Neef et al., 2014), and endophilin act in concert, e.g. through endophilin-mediated dynamin recruitment, remains to be examined. Furthermore, I observed a shift towards larger coated structures in mutants missing two endophilin 
genes. In endophilin 1-SKOs, I observed significantly more CCV s that however had a comparable size to CCV s in Wt IHCs. In contrast, both DKOs harbored more coated vacuoles but unchanged numbers of CCVs compared to Wt. Thus, the uncoating of the CCVs seems already impaired in absence of oneendophilin gene, whereas depletion of two endophilins is required to generate changes in the size of the coated structures. Whether the increased occurrence of coated vacuoles originates from disturbances in the fission process or from impaired membrane bending, comparable to what has been observed in absence of CALM, cannot fully be elucidated at this point. Accumulations of coated structures especially in both examined endophilin DKOs speak against a rate-limiting role of endophilin in membrane bending prior to the formation of the CCPs. However, it cannot be ruled out that more severe impairments of fission and uncoating mask potential defects in the stabilization of membrane curvature in the endophilin mutants in IHCS.

\subsubsection{BULK-LIKE MEMBRANE RetRIEVAL}

Several studies have shown that IHC s are capable of performing bulk-like endocytosis, meaning that ELV s are formed through invaginations of the cell membrane after stimulation (Chakrabarti et al., 2018; Kamin et al., 2014; N eef et al., 2014; Revelo et al., 2014). Electrophysiological recordings revealed that strong stimulation induces an exponential $\mathrm{C}_{\mathrm{m}}$ reduction that has been associated with bulk endocytosis (M oser and Beutner, 2000; N eef et al., 2014). M oreover, after several minutes of $\mathrm{K}^{+}$ stimulation (Fig. 2-9), the number of ELVs, which can be heterogenous in size and partially contain budding CCPs, was shown to be increased drastically (Jung et al., 2015b; N eef et al., 2014). Even though 15 min of continuous stimulation appear harsh, IHCs did not show depletions of SV pools in particular at the ribbon. It seems as if IHCs are prepared for these continuous stimulation conditions. Physiologically, the bulk-like membrane retrieval may be required for mammals to repetitively perceive sound stimuli of the same frequency over a course of several seconds or even minutes. $N$ ot only the faster kinetics compared to CM E, but also the greater volume of the internalized membrane, as well as the better accessibility of internalized membranes for cytosolic proteins involved in SV reformation, may display advantages of bulk-like membrane retrieval (see 4.1.4 Do IHCs Need Clathrin-mediated Endocytosis?). 


\subsubsection{Bulk Retrieval May Sufficiently ReCover Membranes In AbSenCE Of AP180}

In AP- $2 \mu \mathrm{KO}$ mice, increased numbers of ELV s have been observed after stimulation (Jung et al., 2015b). The authors argued that increased membrane tension and/ or accumulations of stranded proteins led to elevated levels of bulk-like membrane retrieval. In contrast to these findings, I did not observe more ELV s in AP180-KO; however, the ELV volume was on average increased (see Fig. 3-4). Not only different mechanisms or differing relevance of the two adaptor proteins for IHC endocytosis, but also the different technical approaches may have caused the discrepancy regarding the ELV numbers between thetwo studies. I performed electron tomography to accurately determine ELV numbers and volumes in absence of AP180, whereas in the AP-2 study, only numbers of ELVs were counted. It is therefore possible, that ELV s were also enlarged in AP- $2 \mu \mathrm{KO}$ mice. Larger ELV s may even have influenced the counts of these structures, as the probability of cutting through an ELV increases with the size of the respective ELV. A direct comparison of ELV numbers may therefore not be meaningful at this point. However, what can surely be concluded, is, that after $5 \mathrm{~min}$ of continuous stimulation, defects in A P180-mediated clathrin recruitment did not elevate the number of bulk internalization events significantly compared to stimulated Wt (see Fig. 3-5). It is likely that the increased ELV volume results from a prominent role of AP180 in SV reformation, as I will more comprehensively discuss in the chapter 4.1.3 Endophilin and AP180 are Involved in SV reformation.

\subsubsection{EndoPhilin May HaVea Role In CLATHRIN-INDEPENDENT ENDOCYTOSIS IN IHCS}

In random sections from endophilin mutants, I observed that a larger area of the section within $1 \mu \mathrm{m}$ around the synaptic ribbon is covered by ELVs. This mainly resulted from larger ELVs, but also from a higher number of ELV s at least in 1/2-DKO (see A ppendix Fig. S2). On the one side, the larger ELV area observe in both, AP180 and endophilin mutants, could be explained by disturbed clathrindependent SV reformation, as discussed in more detail in the following section. On the other side, endophilin may play a regulatory role in the fission of bulk-like membrane invaginations. Previous studies have shown that endophilin, dynamin and actin jointly act in the fission of membranous tubular invaginations in non-neuronal mammalian cells (Boucrot et al., 2015; Renard et al., 2015). And also in neuronal ADBE, dynamin and actin-nucleating formins were shown to play an essential role (Soykan et al., 2017). During UFE in neurons, endophilin likewise mediates the recruitment of dynamin to the neck of endocytic vesicles with a volume equal to several SV s (W atanabe et al., 2018). In this process, absence of endophilin does not completely block fission but only affects the kinetics 
of dynamin-mediated fission of endocytic vesicles. Apart from the dynamin-mediated fission processes described in neuronal and non-neuronal clathrin-independent endocytic pathways, a recent study has examined a dynamin-independent mechanism (Simunovic et al., 2017): here, NBAR domain proteins like endophilin, together with cytoskeletal and/ or motor proteins, enable the scission of membrane tubes. Thus, also in IHCS, endophilin may be involved in the scission of membranous invaginations, either via directly mediating the scission process, or via recruitment of dynamins. In the here presented study, I observed changes especially in the size of ELV s, that can not only be attributed to impaired SV reformation, but also to defects in the constriction of membrane invaginations. Although the fission of the narrow neck of such a membrane invagination is probably not visible during patch-clamp recordings ( $\mathrm{N}$ eef et al., 2014), we further observed a strong trend towards a slower $\mathrm{C}_{\mathrm{m}}$ decline in the exponential phase of endocytosis in both endophilin DKOs. This reduction may display a general slowdown of the internalization process prior to fission. It is therefore possible, that endophilin acts as a wider-span regulator of bulk-like membrane retrieval in IHCs including e.g. the fission process and/ or preceding steps. H owever, the effects of knocking out two endophilin genes on the exponential $\Delta \mathrm{C}_{m}$ after $200 \mathrm{~ms}$ stimulation weremild. This indicates that one remaining endophilin gene could still be sufficient. Alternatively or in addition, endophilinindependent forms of bulk endocytosis may exist in IHCs.

To date, little is known about the mechanisms of bulk-like retrieval in IHCS, except that it appears to be activity-dependent ( $N$ eef et al., 2014) and that bulk-like membrane retrieval can probably compensate very high rates of SV exocytosis. Even if endophilin is involved in the formation and/or fission of membranous invaginations, we do not know if the mechanisms in IHCs are similar to $A D B E$, to neuronal UFE or to mechanisms in non-neuronal cells. Furthermore, different combinations of the afore-mentioned mechanisms are conceivable. Future studies will be required to identify further key players in bulk-like membrane retrieval in IHCs. Due to their co-actions with endophilin, actin and actin-modifying proteins like formins, as well as dynamins, serve as interesting candidate proteins (Boucrot et al., 2015; Renard et al., 2015; Soykan et al., 2017). M oreover, patchclamp experiments at physiological temperature will be required, as UFE could not be detected at room temperature in neurons (W atanabe et al., 2018).

Additionally, determining the molecular composition of ELVs in the IHC base, where the ribbon synapses are located, may be a promising approach. M arkers for early endosomes (e.g. EEA 1) as well as for late endosomes (VAM P7) or lysosomes (Lamp1) have exclusively been detected in the apical, 
ribbon-free half of the IHC (Revelo et al., 2014; own observations). W hether ELV s seen in the base of IHCs all derive from bulk-like membrane retrieval or whether a fraction of those ELV s is made up by bona fide endosomes is not clear to date. N either has been investigated yet in detail if phagosomes, which may also appear as round- to oval-shaped cytosolic membranous vacuoles in EM sections (de Chastellier, 2008), can be detected in the IHC base. A possible (alternative) explanation for the slight increase in the number of ELV s in the endophilin 1/2-DKOs, but not in 1/3-DKOs or 1-SKOs, and neither in AP180-KOs, could be that protein homeostasis is more severely disturbed in those mutants. A recent study revealed that endophilin is involved in autophagy, whereby absence of endophilins results in cell death and neurodegeneration (M urdoch et al., 2016). The authors could show that apoptosis rates were elevated in the motor cortex and hippocampus of TKOs short after birth and in 1/2-DKOs at P18, but only at 18 months in 1/3-DKOs. Furthermore, 1/2-DKOs suffer from a general growth delay, major neurological and motor impairments as well as from epileptic seizures and ataxia. Even though synaptic function was not severely impaired in those 1/2-DKOs at P15-P18, one may speculate that first signs of disturbed protein homeostasis have also arisen in IHCS at that age. More precisely, membranous structures involved in protein degradation may have formed, that are morphologically indistinguishable from ELVs. However, these assumptions are highly speculative and future studies including membrane markers like mCLING as well as mRNA/ protein analyses of autophagy markers will be required to test if protein homeostasis indeed starts to be disturbed in IHCs from two weeks old 1/2-DKOs.

\subsubsection{AP180 AND ENDOPHILIN ARE INVOLVED IN SV REFORMATION}

A part from the changes in number and/ or size of coated structures and the size of ELVs, also numbers of SV s have been affected by the absence of endophilins or AP180. Numbers of SV s in ribbon proximity in endophilin 1/2-DKOs were approx. 34\% reduced (analysis from random sections), whereas SV numbers in AP180-KOs were more than $60 \%$ lower when compared to Wt (examined from tomograms). Electron tomography following $5 \mathrm{~min}$ high- $\mathrm{K}^{+}$stimulation revealed that multiple CCPs can bud from a single ELV in IHCs. Similar observations have previously been made after 2 min $\mathrm{K}^{+}$stimulation at physiological temperature followed by conventional embedding and analysis of random sections ( $\mathrm{N}$ eef et al., 2014). In endophilin-KOs, the number of coated pits at ELV s was even higher than in Wt. In contrast, the number of ELVs with budding pits was much lower in AP180-KOs. Furthermore, if an ELV in an AP180-KO IHC contained CCPs, then usually one, and in few cases two. It is therefore likely that both proteins are required for clathrin-dependent 
SV reformation following bulk-like membrane retrieval, whereby AP180 recruits clathrin to the ELV and endophilin mediates fission and probably also uncoating.

The strong reduction of SV numbers in AP180-KOs on the one side and the (almost) unaffected kinetics of slow endocytosis likely representing CME on the other side indicate that clathrindependent processes are more important for the reformation of SV $s$ than for the restoration of cell membranes after exocytosis. The adaptor protein-mediated recruitment of clathrin seems to depend on the exocytic proteins but not on thetargeted membrane itself (either the cell membraneor ELVs), which indicates that mechanisms of CME and of clathrin-dependent SV reformation may be overlapping or even identical. Especially during strong, continuous stimulation, bulk-like retrieval seems more effective in clearing release sites from exocytic proteins (Gimber et al., 2015; N eher, 2010) and may thus be favored in IHCs. Indeed, we observed morphological alterations regarding ELV size and numbers of CCPs at ELV s in absence of AP180 and also AP-2, whereas physiological recordings revealed unchanged kinetics of the slow mode of endocytosis likely reflecting CME. Consequently, both adaptor proteins seem to be essential for clathrin-dependent SV reformation following bulklike membrane retrieval in IHCS, as well as after ADBE or UFE in neurons (Jung et al., 2015b; Kononenko et al., 2014; Koo et al., 2015; N eef et al., 2014; W atanabe et al., 2014). H owever, also yet to be identified clathrin-independent mechanisms of SV reformation following e.g. bulk endocytosis may be active not only in neurons (for review see M ilosevic, 2018) but also in IHCS.

\subsubsection{Do IHCs NeEd CLATHRIN-MediATEd ENDOCYTOSIS?}

Given that IHCs show very high rates of continuous SV turnover (Pangršič et al., 2010), one may hypothesize that CME, which is theslowest known mode of endocytosis in cells with synaptic activity, plays a minor role in IHC membrane retrieval. In neurons, the role of CME has likewise been questioned: several studies have instead identified UFE followed by subsequent clathrin-dependent SV reformation - and not CME - as the predominant path of SV recycling at least after mild stimulation (D elvendahl et al., 2016; Kononenko et al., 2014; W atanabe et al., 2013; W atanabe et al., 2014).

Still, CCPs at the plasma membrane of IH C s have been observed previously (Frank et al., 2010; Jung et al., 2015b; N eef et al., 2014; Sendin et al., 2007; Siegel and Brownell, 1986) and also in the studies presented here. These pits serve as a strong indicator for the capability of IHCs to inherently perform CME. Yet, the number of CCPs was per se low compared to the number of CCV s and not much 
elevated in Wt mice after 5 min (for AP180, in combination with H PF) or 15 min (for endophilin, in combination with chemical fixation) of $\mathrm{K}^{+}$stimulation compared to resting condition. In endophilin DKO mutants, the number of CCPs at the plasma membrane was slightly higher compared to $\mathrm{Wt}$ in resting condition but not additionally increased upon stimulation despite the deficits in fission. In A P180-KO mice, I did not observe any significant changes in the number of CCPs compared to $\mathrm{Wt}$ in the resting condition and after stimulation. M oreover, patch-clamp recordings revealed unaltered endocytosis rates in AP-2 $\mu \mathrm{KO}$ s (J ung et al., 2015b) or only slight disturbances in either the return to baseline or theslope of the linear component in endophilin and fitful dynamin-KO mice as well as in IHCs treated with the clathrin blocker pitstop 2 ( $N$ eef et al., 2014).

So, how physiologically relevant is CM E for IHCs? Primarily, unaltered endocytosis rates in AP- $2 \mu$ $\mathrm{KO}$ mice and unchanged numbers of CCPs in AP180-KO mice point towards very low levels of CM E that can be compensated if just one of the adaptor proteins is present (in contrast to the AP-2 and A P180-dependent reformation of SVs), or by a redundancy of CM E in membrane retrieval in IHCS. One may argue that exocytosis-induced increased membrane tension and/ or membrane-stranded proteins, that are recycled via CME in Wt, slightly increase bulk-likemembrane retrieval of uncoated membrane compartments, and thus stabilize endocytosis rates in absence of AP-2 or AP180. H owever, since the number of ELV s in AP180-KO mice was neither increased in resting condition nor after stimulation, the rates of such a compensatory mechanism, and hence the impact of CME, would probably be low.

N otably, endophilin and dynamin become active when a coated pit is already formed, whereas AP-2 and AP180 are required for the early steps of CME. O ne may argue that the formation of CCPs helps to decrease membrane tension via small invaginations that are still connected to the plasma membrane. This way, bulk-like endocytosis would less likely be induced in absence of endophilin compared to AP- $2 \mu$ and A P180-KO mice, albeit slow endocytosis appears impaired. H owever, slower $\mathrm{C}_{m}$ reductions after application of pitstop2 speak against this hypothesis ( $\mathrm{N}$ eef et al., 2014). M oreover, previous studies have shown that endophilin and/ or dynamin are involved in clathrin-independent forms of endocytosis like bulk retrieval and UFE in neurons as well as in non-neuronal mammalian cells (Boucrot et al., 2015; Renard et al., 2015; Simunovic et al., 2017; Soykan et al., 2017; W atanabe et al., 2018). In consideration of the above-noted observations, one may presume that endophilin, and possibly also dynamin, are also involved in clathrin-independent forms of endocytosis in IHCS (see 4.1.2.2 Endophilin M ay Havea Rolein Clathrin-independent Endocytosis in IHCs). H owever, this 
form of endophilin-dependent bulk-like membrane retrieval is probably either of limited capacity or can easily be compensated, since a trend towards only mild impairment of the exponential phase of endocytosis has been observed in absence of endophilin. Notably, recent studies have questioned the specificity of the clathrin blocker pitstop 2 (Dutta et al., 2012; W illox et al., 2014), indicating that the slight impairments in the slow mode of endocytosis seen in N eef et al., 2014 may be ambiguous. These findings indicate, that the deficits in the slow mode of endocytosis, observed in absence of endophilins and dynamin, as well as after pitstop 2 application, cannot with certainty be related to deficits in CME only, but may also result from deficits in clathrin-independent forms of endocytosis.

Together with the observations made in AP- $2 \mu$ and AP180-KO mice, one may therefore conclude that CME has a minor relevance for membrane retrieval following exocytosis at least under our experimental conditions. After moderate to strong stimulation, bulk-like membrane-retrieval followed by clathrin-dependent SV reformation seems to be the preferred pathway. However, recordings of IHC endocytosis have so far been performed at room temperature. Not only the detectability of UFE but also the kinetics of clathrin-dependent steps seem to dependent highly on the temperature (Delvendahl et al., 2016; N ouvian, 2007; Renden and von Gersdorff, 2007; Soykan et al., 2017; Watanabe et al., 2013). Therefore, it is necessary to perform studies at physiological temperature to comprehensively re evaluate the role of $\mathrm{CME}$ in IHC endocytosis. Even though the physiological relevance of CME for IHC endocytosis may at least be questioned, this mechanism could still be required for the long-term restoration of cell membranes in IHCs. M ore precisely, CME could be essential for the slow recycling of cargo stranded at the plasma membrane after exocytosis, as previously reported for neurons (Gimber et al., 2015; Soykan et al., 2017). Here, binding motifs at adaptor proteins for clathrin and $\mathrm{PI}(4,5) \mathrm{P}_{2}$ on the one side and for exocytic proteins on the other side seem to facilitate release site clearance and clathrin-dependent protein sorting (see 4.2.1 AP180 M ay Play A Role Downstream of Docking in IHCS). 


\subsection{AP180 AND ENDOPHILIN ACT BEYOND SV RECYCLING}

Both here presented studies indicate that endophilin and AP180, both originally described as key players in different steps of CME in neurons (Lindner and U ngewickell, 1992; M ilosevic et al., 2011; M orris et al., 1993; Ringstad et al., 1997; Schuske et al., 2003; Verstreken et al., 2002), may act in processes beyond classical endocytosis. To our surprise, we detected a mild, but significant impairment of sustained exocytosis in IHCs of endophilin 1/3-DKOs, that could not entirely be explained by the small concomitant decrease in $\mathrm{Ca}^{2+}$-currents. It is unlikely that the smaller changes in $C_{m}$ after 200 ms stimulation resulted exclusively from impaired endocytic capabilities, as e.g. the number of cytosolic SV s in endophilin 1/3-DKO s has been unchanged compared to Wt. M oreover, even after UFE, the reformation of SV s requires approx. 20 s (W atanabe et al., 2013). Consequently, defects in endocytosis and/ or SV reformation alone cannot explain the slight deficits in exocytosis already after $200 \mathrm{~ms}$. Previous studies have shown that problems in SV replenishment, in scaffolding of $\mathrm{CAZ}$ proteins and $\mathrm{Ca}^{2+}$-channels, as well as in release site clearance can lead to impaired sustained exocytosis in IHCs (Jung et al., 2015b; Krinner et al., 2017; Pangršič et al., 2010; Vogl et al., 2015). In endophilin 1-SKOs as well as in 1/3-DKOs, $\mathrm{Ca}^{2+}$-influx was impaired. STED microscopy of Cav 1.3 channels revealed a reduction of the area of $\mathrm{Ca}^{2+}$-channel clusters beneath the ribbon in those two mutants that nicely matches the reduced $\mathrm{Ca}^{2+}$-influx. H owever, the decrease in sustained exocytosis was beyond what would be expected from the impaired Ca+influx in the 1/3-DKO mutants. Additionally, I observed morphological changes in AP180-KOs that cannot be explained by disturbed endocytosis alone. The high structural preservation that we received from H PF and freeze substitution combined with electron tomography allowed me to analyze tethers connecting M P-SV s to the plasma membrane. After 5 min of $\mathrm{K}^{+}$stimulation, I detected a higher number of multi-tethered SV s and docked SV s in AP180-KOs compared to Wt (Fig. 3-5). Comparable changes in tether formation have previously been brought into connection with deficits in sustained exocytosis in IHCs of otoferlin mutant mice (Chakrabarti et al., 2018).

Interestingly, endophilin- and AP180-KOs differed in the distribution of SVs in the different morphological SV pools: 1/3-DKOs possessed a relatively lower number of RA-SV s than cytosolic SV s compared to the respectiveSV pools in W $\mathrm{t}$. In AP180-K O mice it has been theother way around: Here, the number of SV s in the cytosol has been drastically reduced in resting condition as well as after stimulation. RA-SV s and M P-SV s were only slightly (20\%) lower compared to Wt in resting 
condition and unchanged after stimulation. It is therefore likely that different factors have led to the deficits in sustained exocytosis in endophilin mutants and to morphological observations suggesting deficits in exocytosis in AP180-KO mice.

\subsubsection{AP180 MAY PLAY A ROLE DOWNSTREAM OF DOCKING IN IHCS}

In inhibitory neurons, which show a more tonic release than excitatory neurons, three mechanisms were found to be affected by AP180 absence, ultimately leading to impaired exocytosis (Koo et al., 2015): (i) The binding of AP180 to VAM P2 and to other members of the VAM P family was shown to be required for protein sorting after SV fusion (Koo et al., 2011; Koo et al., 2015; M iller et al., 2011). Elevated levels of surface-stranded VAMP2 in neurons lacking AP180 indicated that the adaptor protein has a physiological role in release site clearance (Koo et al., 2015). (ii) Via its ANTH domain, A P180 specifically binds to the SNARE domain of VAM P2 after the NSF-mediated disassembly of the SNARE complex (see Fig. 1-5; Koo et al., 2011; M iller et al., 2011). This way, AP180 prevents VAM P2 from the unwanted re-binding of other SNARE proteins post-fusion and enables efficient SV priming. (iii) In neurons of AP180-KO mice, impaired protein sorting led to reduced copy numbers of VAM P2 at SV s (K oo et al., 2015). In neurons of W t mice, SV s contain roughly 70 VA M P2 molecules (Takamori et al., 2006; Wilhelm et al., 2014), although only few SN A RE complexes were shown to be required for SV fusion (M ohrmann et al., 2010; Sinha et al., 2011). A recent study has suggested that the high number of SNARE proteins is required to stabilize the formation and thesize of the fusion pore at least in vitro (Bao et al., 2018).

All these potential defects in priming, fusion and release site clearance are a result of the interaction of AP180 with VAM P2. Although IH C s seem to operate without VAM P2 and other neuronal SNARE proteins (N ouvian et al., 2011), I observed morphological changes in IH Cs from AP180-KO mice that may likewise be attributed to deficits downstream of SV docking. The moderate reduction of SV numbers around the ribbon compared to the strong reduction in the cytosol indicates that SV s can be recruited to the release sites in absence of A P180, but they cannot fuse as easily as in Wt. M oreover, I observed a significantly higher number of multi-tethered MP-SVs and a trend towards more morphologically docked SV s in theKO, whereas the number of single-tethered M P-SV s was reduced. It has recently been suggested that the distance between AZ membrane and SV s is on average shorter within the group of multi-tethered SV s than within single-tethered or untethered SV s (Chakrabarti et al., 2018; Fernández-Busnadiego et al., 2013), indicating that the formation of multiple tethers 
between SV s and AZ membrane is a prerequisite for fusion. If one or more steps downstream of docking are impaired, SV s consequently accumulate in a preceding state, i.e. one in which they are brought close to the AZ membrane via multiple tethers. Chakrabarti et al., 2018 recently examined filamentous tethers in pachanga mutants which harbor a point mutation in the $\mathrm{C}_{2} \mathrm{~F}$-domain of otoferlin and show impairments particularly in the sustained phase of exocytosis (Pangršič et al., 2010). Comparable to my observations in AP180-KO mice, they detected increased numbers of multi-tethered and docked SV s in IH C s of pachanga mutants, which have been explained by potential deficits in priming or release site clearance (Chakrabarti et al., 2018).

It is therefore tempting to speculate that AP180 also plays a role in the sorting of a yet unknown exocytic protein in IHCS. In this scenario, absence of AP180 would lead to accumulations of this exocytic protein at the AZ membrane, comparable to the obersved changed distribution of otoferlin in IHCs in absence of AP-2 (Jung et al., 2015b). Protein agglomerations at the AZ membrane could form a barrier for SV s to come close to the AZ membrane, which would then lead to the observed increased average distance of M P-SV s to the AZ membrane in AP180-KO mice in resting condition (see Fig. 3-4). Less efficient sorting could further reduce the before-mentioned stabilization of the fusion pore, leading to higher numbers of M P-SV s in stages preceding SV fusion. One point that speaks for the presence of yet to be identified exocytic proteins, which may even be related to neuronal SNARE proteins, is the capability of otoferlin to bind to t-SNAREs in a Ca $\mathrm{C}^{2+}$-dependent manner (H ams et al., 2017; Ramakrishnan et al., 2009; Roux et al., 2006).

For the moment, the here presented speculations cannot further be substantiated. Exocytic recordings following different stimulation durations, as well as with lower (or higher) $\mathrm{Ca}^{2+}-$ concentrations than used in standard protocols, and ideally in combination with postsynaptic recordings, may help to detect potential changes in fusion, also including fusion pore flickering. A thorough characterization of the hearing phenotype, including not only ABR but also single-unit recordings, may be required to elucidate the physiological relevance of the morphological changes observed in theIHCs from AP180-KO mice. Confocal microscopy and biochemical studies may lead to the identification of a potential exocytic protein sorted by AP180 in IHCS. 


\subsubsection{ENDOPHILIN REGULATES THE SIZE OF CAv1.3 CLUSTERS AND INTERACTS WITH OTOFERLIN}

We detected slight reductions in $\mathrm{Ca}^{2+}$-influx in endophilin 1-SK Os as well as in 1/3-DKOs. However, only in 1/3-DKOs, sustained exocytosis was concomitantly reduced. Ratios of $\mathrm{Ca}^{2+}$-influx and $\Delta \mathrm{C}_{\mathrm{m}}$ revealed that the observed reduction in exocytosis after 200 ms of stimulation was stronger than what would be expected from the reduced $\mathrm{Ca}^{2+}$-influx. Therefore, the reduced $\mathrm{Ca}^{2+}$-influx alone cannot explain the observed deficit in sustained exocytosis. Still, this finding is remarkable: We found out, that the $\mathrm{Ca}^{2+}$ phenotype results from smaller $\mathrm{Ca}^{2+}$-channel clusters in those mutants, as examined using STED microscopy (Fig. 2-3). So far, the interaction of endophilins and voltage-gated $\mathrm{Ca}^{2+}$ channels has been ascertained only via biochemical studies (Chen et al., 2003). W e could now for the first time to our knowledge substantiate that endophilins regulate the formation of $\mathrm{Ca}^{2+}$-channel clusters in vivo. M oreover, the inactivation of $\mathrm{Ca}^{2+}$-channels was increased in TKO $\mathrm{s}$ in organotypic cultures, indicating that endophilin may have an indirect or even a direct role in the functional modulation of Cav1.3 channels.

In addition to the interaction with $\mathrm{Ca}^{2+}$-channels, we substantiated an interaction of endophilin-A 1 and otoferlin, paired with a mild reduction of otoferlin levels (approx. 30\%) and unchanged otoferlin distribution in absence of endophilin A1 and A3 (Fig. 2-5). A bsence of the endocytic adaptor protein AP-2 has previously been shown to likewise result in impaired sustained exocytosis, paired with a strong reduction of otoferlin levels (Jung et al., 2015b). In a rescue experiment, AP-2 levels were restored, while otoferlin levels were elevated to $50 \%$ of W t otoferlin levels (Jung et al., 2015b). These experiments showed that al ready those relatively low otoferlin levels were sufficient for maintaining normal exocytosis rates (Jung et al., 2015b). It is therefore unlikely that the observed slight otoferlin reductions in the endophilin 1/3-DKOs could explain the observed impairments in sustained exocytosis.

Instead, interactions of $\mathrm{Ca}^{2+}$-channels and endophilin as well as of otoferlin and endophilin could be relevant for the coupling of exo- and endocytosis. Otoferlin could for example not only recruit AP-2 to the plasma membrane after fusion (Jung et al., 2015b) but also endophilin. Beyond that, $\mathrm{Ca}^{2+}-$ channels, otoferlin and endophilin could form a network within the CAZ, that is required for the tight balancing of the different steps within the SV cycle. Indeed, $\mathrm{Ca}^{2+}$ could directly or indirectly induce endophilin-dependent endocytosis in IHCS, since previous studies have shown that $\mathrm{Ca}^{2+}$ is 
involved in the coupling of exo-and endocytosis not only in IHCs but also at other ribbon and CNS synapses (Beutner et al., 2001; Hosoi et al., 2009; Neves et al., 2001; Wu et al., 2009). The slightly reduced rates in the exponential phase of endocytosis in endophilin DKOs could serve as another indicator for the impaired coupling of exo- and endocytosis. H owever, more studies will be required to examine such a potential interplay of endophilin, otoferlin and $\mathrm{Ca}^{2+}$-channels as well as the $\mathrm{Ca}^{2+}$ dependence of endophilin-dependent endocytosis in IHCs.

\subsubsection{ENDOPHILIN MAY ACT IN SV RESUPPLY}

Not only defects in release site clearance, but also disturbed SV replenishment, and more generally, the recruitment of SV s to the ribbon and/ or the AZ, serveas alternative explanations for theimpaired sustained exocytosis observed in endophilin 1/3-DKOs (J ean et al., 2018; Jung et al., 2015a; Jung et al., 2015b; Pangršič et al., 2010; Strenzke et al., 2016). In this DKO, the number of SV s was reduced at the ribbon but not in the cytoplasm, indicating that the recruitment of SV s to the release sites is more likely affected than docking, priming or fusion (as elucidated for AP180). Interestingly, we made similar observations in chromaffin cells from adrenal gland tissue of endophilin TKOs (Gowrisankaran et al., unpublished) and, though less severely, in 1/3-DKOs. In those mutants, the number of morphologically docked LDCVs was unchanged, whereas fewer LDCV s were present between 5 and $10 \mathrm{~nm}$ away from the plasma membrane. Like in IHCs, neurotransmitter release was impaired in chromaffin cells of TKOs, while exocytic proteins like VAM P2, SNA P-25 and M unc-18 were unaffected. Therefore, reasons for impaired SV recruitment in IHCs and chromaffin cells of endophilin mutants could be problems in the maturation of vesicles, including loading of neurotransmitters and equipment of cargo proteins, or impairments in the transport of vesicles to the release sites. In IHCS, distribution and levels of the glutamate transporter $\mathrm{V}$ glut3 were unaltered in 1/3-DKOs, as qualitatively assessed from immunohistochemical stainings. Thus, at least in IHCS, we did not find any obvious indications for deficits in the maturation of SV s.

In central synapses, actin plays a key role not only in multiple steps of exo- and endocytosis, but also in the recruitment of SV s to release sites (M orales et al., 2000; Sakaba and N eher, 2003; V itale et al., 1995). Likewise, F-actin was shown to be required for (sustained) exocytosis in IHCs and in chromaffin cells (Guillet et al., 2016; Trifaró et al., 1992; Vincent et al., 2015). In IH Cs, F-actin forms a meshwork (so-called "cages") around ribbon synapses that probably stabilizes the coordinated recruitment of SV s to the ribbon and regulates exocytosis (Guillet et al., 2016; Vincent et al., 2015). 
In different neuronal and non-neuronal cells, endophilin was shown to either interact with cytoskeletal proteins like actin or to recruit actin-modifying proteins (Ferguson et al., 2009; Simunovic et al., 2017; Soda et al., 2012; Vehlow et al., 2013; Yang et al., 2015). Although these interactions were interpreted as steps in endocytosis, an involvement of endophilin and actin in SV recruitment cannot be excluded. Therefore, endophilin may, either directly or indirectly through interaction with actin-mediating proteins, be required for the organization of F-actin in IHCs and potentially also in chromaffin cells. This way, actin-dependent vesicle recruitment in the different cell types may at least in parts require endophilin.

Involvement of endophilin in this step may further explain the discrepancies in numbers of cytosolic SV s in the different endophilin mutants (Fig. 2-7 and 2-8): In endophilin 1-SKOs, the number of SVs in ribbon proximity may have been increased because SV recruitment has already been slightly impaired whereas endocytosis was mostly unaltered. A recent study has shown that SV s may stay in the cytosol for days or even weeks before they are degraded (Truckenbrodt et al., 2018). This way, SV s that could not be delivered to the ribbon may have accumulated in the 1-SKOs and led to the observed increase in SV numbers. In endophilin 1/2-DKOs, the deficits in endocytosis and/ or SV reformation may have been way more severe than potential deficits in SV recruitment, ultimately leading to reduced numbers of SV s. In 1/3-DKOs, contrary effects of impaired SV reformation and impaired SV recruitment may have together resulted in overall unchanged SV numbers, whereby SV s have been redistributed between cytosol and ribbon.

Taken together, the here discussed studies on the roles of AP180 and endophilin in synaptic transmission revealed that both proteins are involved in endocytosis and/ or in SV reformation. M oreover, slight disturbances in exocytic mechanisms have been observed in AP180 mutants as well as in certain endophilin mutants, however with different steps being affected. AP180 seems to be relevant for release site clearance, possibly via the sorting of an as-yet to be identified IHC protein. Endophilin may beinvolved in therecruitment of SV sto the ribbon, even though a function in release site clearance cannot be ruled out. M oreover, endophilin seems to mediate the coupling of exo- and endocytosis through interactions with otoferlin and possibly also with voltage-gated $\mathrm{Ca}^{2+}$-channels. This way, both proteins have complementary, multi-faceted roles in the synaptic transmission of IHCS. 


\subsection{A Putative Model fOR Synaptic TRANSMISSION In IHCS}

Based on my findings as well as on the results of previous studies, I propose the following model for synaptic transmission in IHCS:
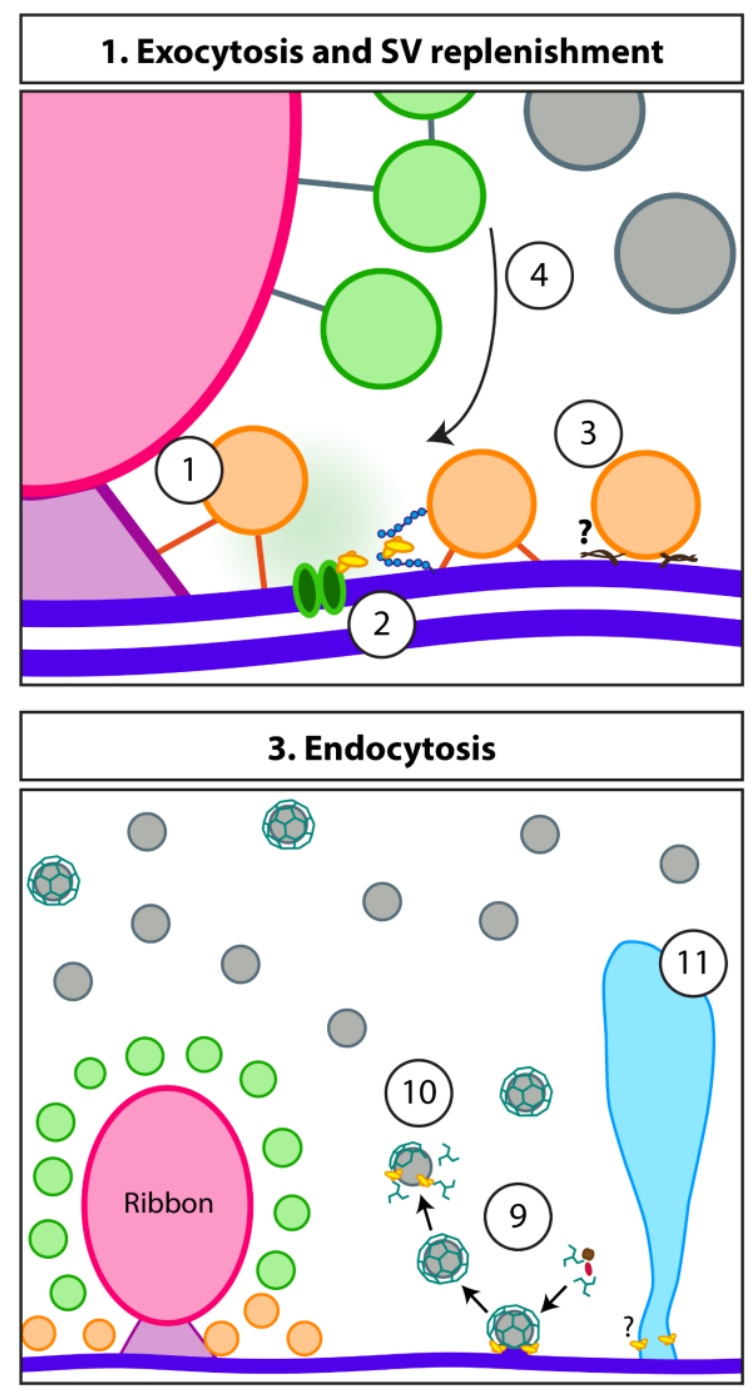

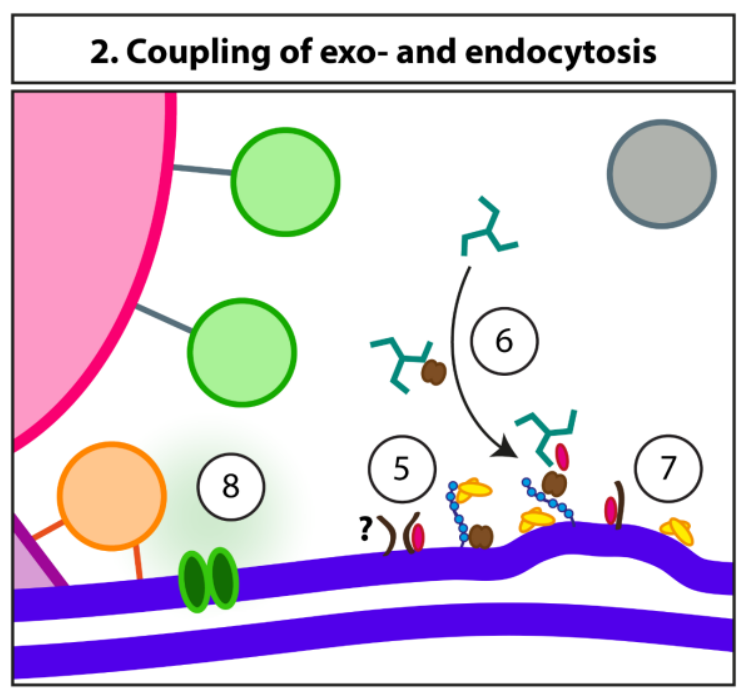

4. SV reformation and recruitment

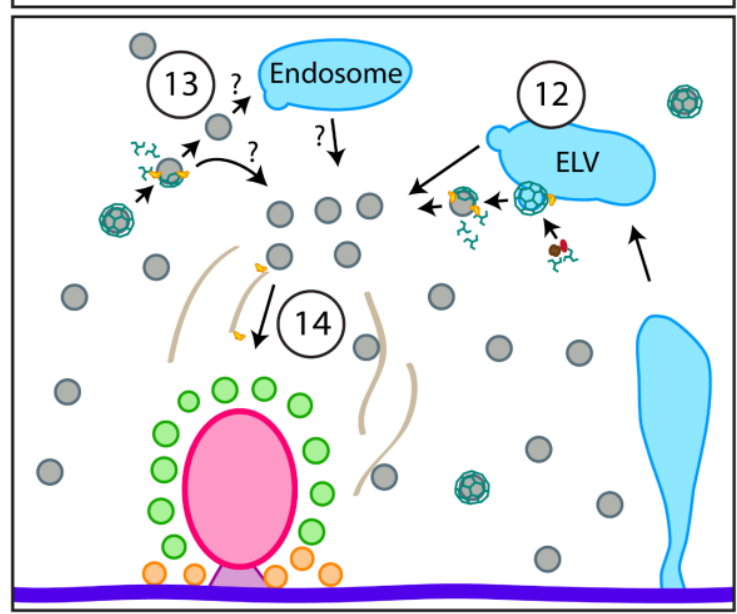

RA-SV cytosolic SV

$00 \mathrm{Ca}_{\mathrm{v}} 1.3$ 80000 Otoferlin

AP-2 X SNARE-related proteins

MP-SV $\sim$ Presynaptic density

* Clathrin \& Endophilin-A

AP180 cytoskeletal proteins like F-actin

(1) Stimulation induces the tethering of MP-SV s to the presynaptic density and to the AZ membrane, which is followed by docking, (priming) and fusion.

(2) $\mathrm{Ca}^{2+}$ enters the cell through $\mathrm{Cav} 1.3$ channels; the formation of $\mathrm{Ca}^{2+}$-channel clusters is promoted by endophilin and CAZ proteins. The tight network of CAZ, otoferlin, endophilin, Cav1.3 and other exo- and endocytic proteins may be required for the efficient coupling of exo- and endocytosis. 
(3) SV fusion is potentially mediated by so far unknown SNARE-related proteins, of which at least one may bind to AP180. Otoferlin may act as $\mathrm{Ca}^{2+}$-sensor and mediates SV fusion.

(4) Sustained release requires replenishment of the RRP, whereby RA-SV s are probably used first and cytosolic SV s later.

(5) After exocytosis, otoferlin and possibly also so far unknown SNARE-related proteins, are prevalent at the plasma membrane.

(6) The coupling of exo- and endocytosis is mediated by adaptor proteins, of which AP-2 binds to otoferlin and AP180 may target a hitherto unknown protein. Concomitantly recruited clathrin is required for the early steps of CME.

(7) Endophilin may further be required for the coupling of exo- and endocytosis, likely via binding to otoferlin. M embrane-bending proteins, potentially including endophilin, induce membrane curvature.

(8) $\mathrm{Ca}^{2+}$ likewise induces endocytosis.

(9) Endophilin is involved in the fission of CCPs from the plasma membrane, possibly together with dynamin. In general, CME may particularly be important for the recycling of SV cargo.

(10) Endophilin mediates the uncoating of CCV s, possibly through the recruitment of uncoating factors like synaptojanin.

(11) Bulk-like endocytosis is prevalent in IHCs and may display a key mechanism especially after moderate to strong stimulation. Endophilin (e.g. together with dynamin and/ or actin) may be involved in the formation and/ or fission of large membrane invaginations.

(12) In a separate step, CCPs are formed at ELV s, which is likely required for SV reformation. Like in CME, AP-2 and AP180 probably recruit clathrin, whereas endophilin is involved in fission and uncoating. Clathrin-independent forms of SV reformation may co-exist in IHCS.

(13) It is not clear what happens after the uncoating of CCV s. N ewly formed small vesicles may either be directly re-loaded and used for exocytosis or fuse with bona fide endosomes. Fusion with endosomes may either be required for protein sorting or for the degradation of defective vesicles. It is neither clear how and if new SV s are later on reformed from endosomes.

(14) Endophilin is potentially involved in the recruitment of SV s from the cytosol to the ribbon. This step may require actin and/ or other cytoskeletal proteins. 


\subsection{FUTURE PERSPECTIVES}

Even though the here presented studies helped to get new insights into the roles of endophilins and AP180 in synaptic transmission, we could not yet prove the suggested roles of those two proteins/ protein families in detail. In the endophilin study, we al ready examined four different combinations of $\mathrm{KO}$. H owever, the fact that three endophilin genes are present in mammals goes along with many more theoretical combinations of endophilin-KOs not all of which we could examine. It would e.g. be interesting to study morphological and physiological changes in endophilin 2- and 3-SKOs, as they would probably allow us to more precisely analyze the specialized or overlapping functions of each endophilin gene.

As already noted in the previous sections, open questions remain regarding endophilin's role in SV recycling. Here, it will be exciting to find out if endophilin can indeed regulate the kinetics of the fission step in clathrin-independent endocytosis. 3D information gathered from either electron tomography or from focused ion beam milling combined with scanning electron microscopy (FIB-SEM ) may help to examine the connectivity of ELV s to the plasma membrane. A part from that, the combination of millisecond-resolution optogenetic stimulation at physiological temperature and HPF may reveal new insights into prevalent modes of endocytosis under different stimulation conditions. This way, the question may be answered if UFE or relatable mechanisms are al so present in IHCS. Moreover, the development of techniques to label membranes and to analyze their path ultrastructurally, either via correlative light and electron microscopy or through electron-dense membrane tags, may help to shed light on the relevance of different endocytic mechanisms in IHCs. They may further help to answer the question of what happens with newly formed vesicles after the uncoating step and how they are transported within the cytosol. This way, one may also be able to further study the potential role of endophilin in SV recruitment to the ribbon.

My studies nicely showed that AP180 is involved in SV reformation following bulk-like membrane retrieval. H owever, we just started to examine the potential role of A P180 in release site clearance. It will be most thrilling to determine the identity of an as-yet unknown potentially exocytic protein targeted by AP180. Since the ANTH domain of AP180 specifically targets a small, conserved helical segment within the SNARE domain of e.g. VAMP2 and VAMP8, the next steps will be to test for potential interactions of AP180 and VAM Ps in IHCs, whereby VAM P1-3 and VAM P7 have already been excluded. Given that the motif of the sequence targeted by AP180 is already known (and 
potentially conserved in IHCs), one may perform single cell mRNA sequencing and screen for proteins containing this motif. Moreover, pull-downs using AP180 protein and a homogenate of organ of Corti tissue combined with mass spectrometry may help to identify the exocytic protein. These experiments may display the first steps in the identification of the hitherto unknown release machinery in IHCS.

I al ready pointed out that endophilin mutants show disturbances in protein homeostasis due to the involvement of endophilin in autophagy. Endophilin 1/2-DKO s may be an interesting candidate to study protein homeostasis in IHCs, as the earliest onset of cellular dysregulations may be expected in those mutants (except from TKOS). A part from the very high rates of SV turnover, I H Cs have to deal with the continuous regeneration of cytomatrix proteins like actin and tubulin, that are not only required for SV transport but also for the maintenance of cell tension and cell polarity, as well as for the stabilization of stereocilia. Therefore, it is likely that protein formation and degradation systems are likewise adapted, which makes I HCs an interesting model to study autophagy. 
Discussion | Future Perspectives 


\section{REFERENCES}

Alés, E., Tabares, L., Poyato, J. M ., V alero, V., Lindau, M . and Alvarez de Toledo, G. (1999). High calcium concentrations shift the mode of exocytosis to the kiss-and-run mechanism. Nat. Cell Biol. 1, 40-44.

Anggono, V. and Robinson, P. J. (2007). Syndapin I and endophilin I bind overlapping proline-rich regions of dynamin I: role in synaptic vesicle endocytosis. Journal of N eurochemistry 102, 931-943.

Assad, J. A., Shepherd, G. M . and Corey, D. P. (1991). Tip-link integrity and mechanical transduction in vertebrate hair cells. Neuron 7, 985-994.

Bai, J., Hu, Z., Dittman, J. S., Pym, E. C. G. and Kaplan, J. M. (2010). Endophilin Functions as a M embrane-Bending M olecule and Is Delivered to Endocytic Zones by Exocytosis. Cell 143, 430-441.

Bao, H., Das, D., Courtney, N. A ., Jiang, Y., Briguglio, J. S., Lou, X., Roston, D., Cui, Q., Chanda, B. and Chapman, E. R. (2018). Dynamics and number of trans-SN ARE complexes determinenascent fusion pore properties. Nature 554, 260-263.

Becker, L., Schnee, M. E., Niwa, M ., Sun, W., M axeiner, S., Talaei, S., Kachar, B., Rutherford, M . A. and Ricci, A. J. (2018). The presynaptic ribbon maintains vesicle populations at the hair cell afferent fiber synapse. eLife Sciences 7 , e30241.

Betz, A., Thakur, P., Junge, H. J., A shery, U., Rhee, J.-S., Scheuss, V., Rosenmund, C., Rettig, J. and Brose, N. (2001). Functional Interaction of the Active Zone Proteins M unc13-1 and RI M 1 in Synaptic V esiclePriming. N euron 30, 183-196.

Beurg, M., Evans, M. G., Hackney, C. M. and Fettiplace, R. (2006). A Large-Conductance Calcium-Selective M echanotransducer Channel in Mammalian Cochlear Hair Cells. Journal of Neuroscience 26, 10992-11000.

Beurg, M ., M ichalski, N ., Safieddine, S., Bouleau, Y., Schneggenburger, R., Chapman, E. R., Petit, C. and Dulon, D. (2010). Control of exocytosis by synaptotagmins and otoferlin in auditory hair cells. J. N eurosci 30, 13281-13290.

Beutner, D., V oets, T., N eher, E. and M oser, T . (2001). Calcium dependence of exocytosis and endocytosis at the cochlear inner hair cell afferent synapse. Neuron 29,681-690.

Boucrot, E., Ferreira, A. P. A., Almeida-Souza, L., Debard, S., Vallis, Y., Howard, G., Bertot, L., Sauvonnet, N. and M cM ahon, H. T. (2015). Endophilin marks and controls a clathrin-independent endocytic pathway. Nature 517, 460-465.

Boumil, R. M., Letts, V. A., Roberts, M. C., Lenz, C., M ahaffey, C. L., Zhang, Z., M oser, T. and Frankel, W . N. (2010). A $M$ issense Mutation in a Highly Conserved Alternate Exon of Dynamin-1 Causes Epilepsy in Fitful Mice. PLoS Genet 6, el001046.

Brandt, A., Striessnig, J. and M oser, T. (2003). CaV 1. 3 channels are essential for development and presynaptic activity of cochlear inner hair cells. J N eurosci 23, 10832-10840.

Brandt, A., Khimich, D. and M oser, T. (2005). Few CaV 1. 3 channels regulate the exocytosis of a synaptic vesicle at the hair cell ribbon synapse. J Neurosci 25, 11577.

Brose, N. (2008). For better or for worse: complexins regulate SN ARE function and vesicle fusion. Traffic 9, 1403-1413.

Butola, T., W ichmann, C. and M oser, T. (2017). Piccolo promotes vesicle replenishment at a fast central auditory synapse. Front. Synaptic Neurosci. 9.

Cao, M ., M ilosevic, I., Giovedi, S. and De Camilli, P. (2014). U pregulation of Parkin in endophilin mutant mice. J. N eurosci. 34, 16544-16549.

Chakrabarti, R., M ichanski, S. and W ichmann, C. (2018). V esicle sub-pool organization at inner hair cell ribbon synapses. EM BO reports e44937.

Chapochnikov, N. M., Takago, H., Huang, C.-H., Pangršič, T., Khimich, D., Neef, J., Auge, E., Göttfert, F., Hell, S. W., Wichmann, C., et al. (2014). Uniquantal Release through a Dynamic Fusion Pore Is a Candidate M echanism of Hair Cell Exocytosis. Neuron 17, 1389-1403.

Chen, H., Fre, S., Slepnev, V. I., Capua, M . R., Takei, K., Butler, M. H., Di Fiore, P. P. and De Camilli, P. (1998). Epsin is an EH-domain-binding protein implicated in clathrin-mediated endocytosis. Nature 394, 793-797.

Chen, X., Tomchick, D. R., Kovrigin, E., Araç, D., M achius, M., Südhof, T. C. and Rizo, J. (2002). Three-Dimensional Structure of the Complexin/SNA RE Complex. Neuron 33, 397-409.

Chen, Y., Deng, L., M aeno-Hikichi, Y., Lai, M., Chang, S., Chen, G. and Zhang, J. (2003). Formation of an EndophilinCa2+Channel Complex Is Critical for Clathrin-M ediated Synaptic V esicle Endocytosis. Cell 115, 37-48.

Cho, L., Li, G. L., von Gersdorff, H . (2011). Recovery from short-term depression and facilitation is ultrafast and Ca2+dependent at auditory hair cells. J Neurosci 31, 5682-5692.

Chow, R. H., von Rüden, L. and Neher, E. (1992). Delay in vesicle fusion revealed by electrochemical monitoring of single secretory events in adrenal chromaffin cells. Nature 356, 60-63. 
Clary, D. O., Griff, I. C. and Rothman, J. E. (1990). SNAPs, a family of N SF attachment proteins involved in intracellular membrane fusion in animals and yeast. Cell 61, 709-721.

Clayton, E. L. and Cousin, M. A. (2009). The molecular physiology of activity-dependent bulk endocytosis of synaptic vesicles. Journal of N eurochemistry 111, 901-914.

Clayton, E. L., Evans, G. J. O. and Cousin, M. A . (2008). Bulk Synaptic Vesicle Endocytosis Is Rapidly Triggered during Strong Stimulation. J. Neurosci. 28, 6627-6632.

Cole, A. A., Chen, X. and Reese, T. S. (2016). A N etwork of Three Types of Filaments Organizes Synaptic Vesicles for Storage, M obilization, and Docking. Journal of N euroscience 36, 3222-3230.

Corey, D. P. and Hudspeth, A. J. (1979). I onic basis of the receptor potential in a vertebrate hair cell. Nature 281, 675-677.

Cremona, O., Di Paolo, G., W enk, M. R., Lüthi, A., Kim, W. T., Takei, K., Daniell, L., N emoto, Y., Shears, S. B., Flavell, R. A., et al. (1999). Essential role of phosphoinositide metabolism in synaptic vesicle recycling. Cell 99, 179-188.

DeCamilli, P. and Jahn, R. (1990). Pathways to regulated exocytosis in neurons. Annu. Rev. Physiol. 52, 625-645.

de Chastellier, C. (2008). EM analysis of phagosomes. M ethods M ol. Biol. 445, 261-285.

Delvendahl, I., Vyleta, N. P., von Gersdorff, H. and Hallermann, S. (2016). Fast, Temperature-Sensitive and ClathrinIndependent Endocytosis at Central Synapses. Neuron 90, 492-498.

Denker, A . and Rizzoli, S. O. (2010). Synaptic V esicle Pools: An U pdate. Front Synaptic N eurosci 2,

Denker, A., Bethani, I., Kröhnert, K., Körber, C., Horstmann, H., W ilhelm, B. G., Barysch, S. V., Kuner, T., N eher, E. and Rizzoli, S. O. (2011). A small pool of vesicles maintains synaptic activity in vivo. Proceedings of the National A cademy of Sciences 108, 17177-17182.

Dick, O., tom Dieck, S., Altrock, W . D., Ammermüller, J., W eiler, R., Garner, C. C., Gundelfinger, E. D. and Brandstätter, J. H. (2003). The Presynaptic Active Zone Protein Bassoon Is Essential for Photoreceptor Ribbon Synapse Formation in the Retina. Neuron 37, 775-786.

Dickman, D. K., Horne, J. A., M einertzhagen, I. A. and Schwarz, T. L. (2005). A Slowed Classical Pathway Rather Than Kiss-and-Run M ediates Endocytosis at Synapses Lacking Synaptojanin and Endophilin. Cell 123, 521-533.

Dittman, J. and Ryan, T. A. (2009). M olecular circuitry of endocytosis at nerve terminals. Annu. Rev. Cell Dev. Biol. 25, 133-160.

Dowling, J. E. (1968). Synaptic organization of the frog retina: an electron microscopic analysis comparing the retinas of frogs and primates. Proc. R. Soc. Lond., B, Biol. Sci. 170, 205-228.

Duncker, S. V., Franz, C., Kuhn, S., Schulte, U., Campanelli, D., Brandt, N., Hirt, B., Fakler, B., Blin, N., Ruth, P., et al. (2013). Otoferlin Couples to Clathrin-M ediated Endocytosis in M ature Cochlear Inner Hair Cells. J. Neurosci. 33, 9508-9519.

Dutta, D., Williamson, C. D., Cole, N. B. and Donaldson, J. G. (2012). Pitstop 2 Is a Potent Inhibitor of ClathrinIndependent Endocytosis. PLoS One 7,.

Edeling, M. A., Smith, C. and Owen, D. (2006). Life of a clathrin coat: insights from clathrin and AP structures. Nat Rev Mol Cell Biol 7, 32-44.

Edmonds, B. W . (2004). Evidence that fast exocytosis can be predominantly mediated by vesicles not docked at activezones in frog saccular hair cells. J Physiol 560, 439-450.

Elmqvist, D. and Quastel, D. M. (1965). A quantitative study of end-plate potentials in isolated human muscle. J Physiol $178,505-529$.

Farsad, K., Ringstad, N ., Takei, K., Floyd, S. R., Rose, K. and Camilli, P. D. (2001). Generation of high curvaturemembranes mediated by direct endophilin bilayer interactions. J Cell Biol 155, 193-200.

Fenster, S. D., Chung, W. J., Zhai, R., Cases-Langhoff, C., V oss, B., Garner, A . M ., Kaempf, U., Kindler, S., Gundelfinger, E. D. and Garner, C. C. (2000). Piccolo, a Presynaptic Zinc Finger Protein Structurally Related to Bassoon. Neuron 25, 203-214.

Ferguson, S. M. and De Camilli, P. (2012). Dynamin, a membrane-remodelling GTPase. Nature Reviews M olecular Cell Biology 13, 75-88.

Ferguson, S. M ., Brasnjo, G., Hayashi, M ., W olfel, M., Collesi, C., Giovedi, S., Raimondi, A., Gong, L.-W ., A riel, P., Paradise, S., et al. (2007). A Selective Activity-Dependent Requirement for Dynamin 1 in Synaptic Vesicle Endocytosis. Science 316, 570-574.

Ferguson, S., Raimondi, A ., Paradise, S., Shen, H ., M esaki, K., Ferguson, A., Destaing, O., Ko, G., Takasaki, J., Cremona, O., et al. (2009). Coordinated Actions of Actin and BAR Proteins Upstream of Dynamin at Endocytic ClathrinCoated Pits. Developmental Cell 17, 811-822.

Fernández-Alfonso, T. and Ryan, T. A . (2004). The kinetics of synaptic vesicle pool depletion at CNS synaptic terminals. Neuron 41, 943-953.

Fernández-Busnadiego, R., Zuber, B., M aurer, U. E., Cyrklaff, M., Baumeister, W . and Lučić, V. (2010). Quantitative analysis of the native presynaptic cytomatrix by cryoelectron tomography. J Cell Biol 188, 145-156. 
Fernández-Busnadiego, R., A sano, S., O prisoreanu, A.-M ., Sakata, E., D oengi, M., Kochovski, Z., Zürner, M ., Stein, V., Schoch, S., Baumeister, W., et al. (2013). Cryo-electron tomography reveals a critical role of RIM 1a in synaptic vesicle tethering. J. Cell Biol. 201, 725-740.

Fettiplace, R. (2017). Hair Cell Transduction, Tuning, and Synaptic Transmission in the Mammalian Cochlea. Compr Physiol 7, 1197-1227.

Flock, A . and Duvall, A. J. (1965). The ultrastructure of the kinocilium of the sensory cells in the inner ear and lateral line organs. J. Cell Biol. 25, 1-8.

Ford, M. G. ., Pearse, B. M . F., H iggins, M. K., V allis, Y., Owen, D. J., Gibson, A., H opkins, C. R., Evans, P. R. and M cM ahon, H. T. (2001). Simultaneous Binding of Ptdl ns (4,5)P2 and Clathrin by AP180 in the N ucleation of Clathrin Lattices on M embranes. Science 291, 1051-1055.

Ford, M. G. J., M ills, I. G., Peter, B. J., V allis, Y., Praefcke, G. J. K., Evans, P. R. and M cM ahon, H. T. (2002). Curvature of clathrin-coated pits driven by epsin. Nature 419, 361-366.

Frank, T., Khimich, D., Neef, A. and Moser, T. (2009). Mechanisms contributing to synaptic Ca2+ signals and their heterogeneity in hair cells. Proc. Natl. A cad. Sci. U.S.A. 106, 4483-4488.

Frank, T., Rutherford, M. A., Strenzke, N., Neef, A., Pangršič, T., Khimich, D., Fejtova, A., Gundelfinger, E. D., Liberman, M. C., H arke, B., et al. (2010). Bassoon and the synaptic ribbon organize $\mathrm{Ca}^{2}+$ channels and vesicles to add release sites and promote refilling. N euron 68, 724-738.

Fuchs, P. A. (2005). Time and intensity coding at the hair cell's ribbon synapse. The Journal of Physiology 566, 7-12.

Gad, H., Ringstad, N., Löw, P., Kjaerulff, O., Gustafsson, J., W enk, M., Di Paolo, G., N emoto, Y., Crum, J., Ellisman, M . H., et al. (2000). Fission and Uncoating of Synaptic Clathrin-Coated Vesicles Are Perturbed by Disruption of Interactions with the SH 3 D omain of Endophilin. N euron 27, 301-312.

Gallop, J. L., Jao, C. C., Kent, H. M., Butler, P. J. G., Evans, P. R., Langen, R. and M cM ahon, H. T. (2006). M echanism of endophilin N-BAR domain-mediated membrane curvature. The EM BO journal 25, 2898-2910.

Gan, Q. and W atanabe, S. (2018). Synaptic V esicle Endocytosis in Different M odel Systems. Front Cell Neurosci 12.

Giachino, C., Lantelme, E., Lanzetti, L., Saccone, S., Bella Valle, G. and M igone, N. (1997). A novel SH 3-containing human gene family preferentially expressed in the central nervous system. Genomics 41, 427-434.

Gimber, N ., Tadeus, G., M aritzen, T., Schmoranzer, J. and H aucke, V. (2015). Diffusional spread and confinement of newly exocytosed synaptic vesicle proteins. Nat Commun 6, 8392.

Glowatzki, E. and Fuchs, P. A. (2002). Transmitter release at the hair cell ribbon synapse. Nat Neurosci 5, 147-154.

Graham, A. and Shimeld, S. M . (2013). The origin and evolution of the ectodermal placodes. J. A nat. 222, 32-40.

Granseth, B., Odermatt, B., Royle, S. J. and Lagnado, L. (2006). Clathrin-M ediated Endocytosis I s the Dominant M echanism of V esicle Retrieval at Hippocampal Synapses. N euron 51, 773-786.

Gray, E. G. and Pease, H. L. (1971). On understanding the organisation of the retinal receptor synapses. Brain Res. 35, 115.

Graydon, C. W ., Cho, S., Li, G.-L., Kachar, B. and Gersdorff, H. von (2011). Sharp Ca2+ N anodomains beneath the Ribbon Promote Highly Synchronous M ultivesicular Release at Hair Cell Synapses. J. N eurosci. 31, 16637-16650.

Graydon, C. W., Zhang, J., Oesch, N. W., Sousa, A. A., Leapman, R. D. and Diamond, J. S. (2014). Passive Diffusion as a M echanism Underlying Ribbon Synapse V esicle Release and Resupply. J. N eurosci. 34, 8948-8962.

Gu, M., Schuske, K., W atanabe, S., Liu, Q., Baum, P., Garriga, G. and Jorgensen, E. M . (2008). M u2 adaptin facilitates but is not essential for synaptic vesicle recycling in Caenorhabditis elegans. J. Cell Biol. 183, 881-892.

Guan, R., H an, D., H arrison, S. C. and Kirchhausen, T. (2010). Structure of the PTEN-like Region of A uxilin, a Detector of Clathrin-Coated V esicle Budding. Structure 18, 1191-1198.

Guillet, M ., Sendin, G., Bourien, J., Puel, J.-L. and N ouvian, R. (2016). Actin Filaments Regulate Exocytosis at the Hair Cell Ribbon Synapse. J. Neurosci. 36, 649-654.

H allermann, S. and Silver, R. A . (2013). Sustaining rapid vesicular release at activezones: potential roles for vesi cletethering. Trends N eurosci 36, 185-194.

Hams, N., Padmanarayana, M., Qiu, W. and Johnson, C. P. (2017). Otoferlin is a multivalent calcium-sensitive scaffold linking SN A REs and calcium channels. Proc. Natl. A cad. Sci. U.S.A. 114, 8023-8028.

Hao, W., Luo, Z., Zheng, L., Prasad, K. and Lafer, E. M. (1999). AP180 and AP-2 interact directly in a complex that cooperatively assembles clathrin. J. Biol. Chem. 274, 22785-22794.

H arata, N., Pyle, J. L., A ravanis, A. M ., M ozhayeva, M., Kavalali, E. T. and Tsien, R. W . (2001). Limited numbers of recycling vesicles in small CNS nerve terminals: implications for neural signaling and vesicular cycling. Trends in Neurosciences 24, 637-643.

H ata, Y., Slaughter, C. A. and Südhof, T. C. (1993). Synaptic vesicle fusion complex contains unc-18 homologue bound to syntaxin. Nature 366, 347-351.

H aucke, V. and Kozlov, M. M . (2018). M embrane remodeling in clathrin-mediated endocytosis. J. Cell. Sci. 131. 
H aucke, V., Wenk, M. R., Chapman, E. R., Farsad, K. and De Camilli, P. (2000). Dual interaction of synaptotagmin with mu2- and alpha-adaptin facilitates clathrin-coated pit nucleation. EM BO J. 19, 6011-6019.

H aucke, V., N eher, E. and Sigrist, S. J. (2011). Protein scaffolds in the coupling of synaptic exocytosis and endocytosis. Nature Reviews N euroscience 12, 127-138.

Helfmann, S., N eumann, P., Tittmann, K., M oser, T., Ficner, R. and Reisinger, E. (2011). The crystal structure of the $\mathrm{C}_{2} \mathrm{~A}$ domain of otoferlin reveals an unconventional top loop region. J. M ol. Biol 406, 479-490.

Henne, W. M ., Boucrot, E., M einecke, M., Evergren, E., Vallis, Y., M ittal, R. and M cM ahon, H. T. (2010). FCH o proteins are nucleators of clathrin-mediated endocytosis. Science 328, 1281-1284.

H euser, J. E. and Reese, T. S. (1973). Evidence for recycling of synaptic vesicle membrane during transmitter release at the frog neuromuscular junction. J. Cell Biol. 57, 315-344.

Hinshaw, J. E. (2000). Dynamin and Its Role in M embrane Fission 1. Annual review of cell and developmental biology 16, 483-519.

H ohendahl, A., Talledge, N ., Galli, V., Shen, P. S., Humbert, F., De Camilli, P., Frost, A. and Roux, A. (2017). Structural inhibition of dynamin-mediated membrane fission by endophilin. Elife 6,.

Hosoi, N., Holt, M . and Sakaba, T. (2009). Calcium Dependence of Exo- and Endocytotic Coupling at a Glutamatergic Synapse. Neuron 63, 216-229.

Howard, J. and Hudspeth, A. J. (1988). Compliance of the hair bundle associated with gating of mechanoelectrical transduction channels in the bullfrog's saccular hair cell. Neuron 1, 189-199.

Imig, C., M in, S.-W ., Krinner, S., A rancillo, M ., Rosenmund, C., Südhof, T. C., Rhee, J., Brose, N . and Cooper, B. H. (2014). The morphological and molecular nature of synaptic vesicle priming at presynaptic activezones. N euron 84, 416431.

Jahn, R. and Fasshauer, D. (2012). M olecular machines governing exocytosis of synaptic vesicles. Nature 490, 201-207.

Jähne, S., Rizzoli, S. O. and Helm, M. S. (2015). The structure and function of presynaptic endosomes. Exp. Cell Res. 335, 172-179.

Jean, P., Lopez dela M orena, D., M ichanski, S., J aime Tobón, L. M ., Chakrabarti, R., Picher, M . M ., N eef, J., Jung, S., Gültas, M ., M axeiner, S., et al. (2018). The synaptic ribbon is critical for sound encoding at high rates and with temporal precision. Elife 7.

Jing, Z. Z., Rutherford, M . A., Takago, H ., Frank, T ., Fejtova, A., Khimich, D., M oser, T. and Strenzke, N. (2013). Disruption of the Presynaptic Cytomatrix Protein Bassoon Degrades Ribbon Anchorage, M ultiquantal Release, and Sound Encoding at the $\mathrm{H}$ air Cell Afferent Synapse. J Neurosci 33, 4456-4467.

Johnson, C. P. and Chapman, E. R. (2010). O toferlin is a calcium sensor that directly regulates SN A RE-mediated membrane fusion. J. Cell Biol 191, 187-197.

Jung, S., O shima-Takago, T., Chakrabarti, R., W ong, A. B., Jing, Z., Yamanbaeva, G., Picher, M. M ., W ojcik, S. M., Göttfert, F., Predoehl, F., et al. (2015a). Rab3-interacting molecules $2 \alpha$ and $2 \beta$ promote the abundance of voltage-gated CaV 1.3 Ca2+ channels at hair cell active zones. Proc. Natl. A cad. Sci. U.S.A. 112, E3141-E3149.

Jung, S., M aritzen, T., W ichmann, C., Jing, Z., N eef, A., Revelo, N. H ., Al-M oyed, H., M eese, S., W ojcik, S. M ., Panou, I., et al. (2015b). Disruption of adaptor protein $2 \mu(A P-2 \mu)$ in cochlear hair cells impairs vesicle reloading of synaptic release sites and hearing. EM BO J. 34, 2686-2702.

Kaempf, N. and Maritzen, T. (2017). Safeguards of Neurotransmission: Endocytic Adaptors as Regulators of Synaptic Vesicle Composition and Function. Front Cell Neurosci 11, 320.

Kamin, D., Revelo, N. H . and Rizzoli, S. O. (2014). FM Dye Photo-O xidation as a Tool for M onitoring M embrane Recycling in Inner Hair Cells. PLOS ONE 9, e88353.

Kantardzhieva, A., Liberman, M. C. and Sewell, W . F. (2013). Quantitative analysis of ribbons, vesicles, and cisterns at the cat inner hair cell synapse: correlations with spontaneous rate. J. Comp. Neurol. 521, 3260-3271.

Khimich, D., N ouvian, R., Pujol, R., tom Dieck, S., Egner, A., Gundelfinger, E. D. and M oser, T. (2005). H air cell synaptic ribbons are essential for synchronous auditory signalling. Nature 434, 889-894.

Kirchhausen, T. (2000). Clathrin. Annual Review of Biochemistry 69, 699-727.

Kittel, R. J., W ichmann, C., Rasse, T. M ., Fouquet, W., Schmidt, M., Schmid, A., W agh, D. A., Pawlu, C., Kellner, R. R., Willig, K. I., et al. (2006). Bruchpilot promotes active zone assembly, Ca2+ channel clustering, and vesicle release. Science 312, 1051-1054.

Klingauf, J., Kavalali, E. T. and T sien, R. W . (1998). Kinetics and regulation of fast endocytosis at hippocampal synapses. Nature 394, 581-585.

Kokotos, A. C. and Cousin, M. A. (2015). Synaptic vesicle generation from central nerve terminal endosomes. Traffic 16, 229-240.

Kononenko, N . L. and H aucke, V. (2015). M olecular M echanisms of Presynaptic M embrane Retrieval and Synaptic Vesicle Reformation. Neuron 85, 484-496. 
Kononenko, N. L., Diril, M. K., Puchkov, D., Kintscher, M., Koo, S. J., Pfuhl, G., Winter, Y., W ienisch, M., Klingauf, J., Breustedt, J., et al. (2013). Compromised fidelity of endocytic synaptic vesicle protein sorting in the absence of stonin 2. Proceedings of the National Academy of Sciences 110, E526-E535.

Kononenko, N. L., Puchkov, D., Classen, G. A., W alter, A. M ., Pechstein, A., Sawade, L., Kaempf, N ., Trimbuch, T., Lorenz, D., Rosenmund, C., et al. (2014). Clathrin/AP-2 mediate synaptic vesicle reformation from endosome-like vacuoles but are not essential for membrane retrieval at central synapses. Neuron 82, 981-988.

Koo, S. J., M arkovic, S., Puchkov, D., M ahrenholz, C. C., Beceren-Braun, F., Maritzen, T., Dernedde, J., Volkmer, R., Oschkinat, H. and H aucke, V. (2011). SN ARE motif-mediated sorting of synaptobrevin by the endocytic adaptors clathrin assembly lymphoid myeloid leukemia (CALM) and AP180 at synapses. Proceedings of the National A cademy of Sciences 108, 13540-13545.

Koo, S. J., Kochlamazashvili, G., Rost, B., Puchkov, D., Gimber, N ., Lehmann, M ., Tadeus, G., Schmoranzer, J., Rosenmund, C., Haucke, V., et al. (2015). Vesicular Synaptobrevin/VAM P2 Levels Guarded by AP180 Control Efficient Neurotransmission. Neuron 88, 330-344.

Krinner, S., Butola, T., Jung, S., W ichmann, C. and M oser, T. (2017). RIM - binding protein 2 promotes a large number of $\mathrm{CaV} 1.3 \mathrm{Ca2+-channels} \mathrm{and} \mathrm{contributes} \mathrm{to} \mathrm{fast} \mathrm{synaptic} \mathrm{vesicle} \mathrm{replenishment} \mathrm{at} \mathrm{hair} \mathrm{cell} \mathrm{active} \mathrm{zones.} \mathrm{Front} \mathrm{Cell}$ Neurosci.

Lange, R. P. J. de, Roos, A. D. G. de and Borst, J. G. G. (2003). Two M odes of Vesicle Recycling in the Rat Calyx of H eld. J. Neurosci. 23, 10164-10173.

Lenzi, D. and von Gersdorff, H. (2001). Structure suggests function: the case for synaptic ribbons as exocytotic nanomachines. Bioessays 23, 831-840.

Lenzi, D., Runyeon, J. W., Crum, J., Ellisman, M. H . and Roberts, W. M. (1999). Synaptic Vesicle Populations in Saccular H air Cells Reconstructed by Electron Tomography. J. Neurosci. 19, 119-132.

Lenzi, D., Crum, J., Ellisman, M. H . and Roberts, W . M . (2002). Depolarization redistributes synaptic membraneand creates a gradient of vesicles on the synaptic body at a ribbon synapse. N euron 36, 649-659.

Li, G.-L., Keen, E., Andor-Ardo, D., Hudspeth, A. J. and von Gersdorff, H. (2009). The Unitary Event Underlying Multiquantal EPSCs at a Hair Cell's Ribbon Synapse. Journal of N euroscience 29, 7558-7568.

Liberman, M. C. (1978). A uditory-nerve response from cats raised in a low-noise chamber. J. Acoust. Soc. Am 63, 442-455.

Liberman, M. C. (1982a). The cochlear frequency map for the cat: Labeling auditory-nerve fibers of known characteristic frequency. The Journal of the A coustical Society of America 72, 1441-1449.

Liberman, M. (1982b). Single-neuron labeling in the cat auditory nerve. Science 216, 1239-1241.

Liley, A. W. and North, K. A. (1953). An electrical investigation of effects of repetitive stimulation on mammalian neuromuscular junction. J. Neurophysiol. 16, 509-527.

Limbach, C., Laue, M. M., W ang, X., Hu, B., Thiede, N., Hultqvist, G. and Kilimann, M. W. (2011). M olecular in situ topology of A czonin/Piccolo and associated proteins at themammalian neurotransmitter release site. Proceedings of the National A cademy of Sciences 108, E392-E401.

Lindau, M. and Neher, E. (1988). Patch-clamp techniques for time-resolved capacitance measurements in single cells. Pflügers A rchiv European Journal of Physiology 411, 137-146.

Lindner, R. and Ungewickell, E. (1992). Clathrin-associated proteins of bovine brain coated vesicles. An analysis of their number and assembly-promoting activity. J. Biol. Chem. 267, 16567-16573.

M aritzen, T. and H aucke, V. (2018). Coupling of exocytosis and endocytosis at the presynaptic active zone. Neurosci. Res. $127,45-52$.

Maritzen, T., Koo, S. J. and Haucke, V. (2012). Turning CALM into excitement: AP180 and CALM in endocytosis and disease. Biol. Cell 104, 588-602.

M asuda, M., Takeda, S., Sone, M., Ohki, T., M ori, H., Kamioka, Y. and M ochizuki, N. (2006). Endophilin BAR domain drives membrane curvature by two newly identified structure-based mechanisms. EM BO J. 25, 2889-2897.

M atthews, G. and Fuchs, P. (2010). The diverse roles of ribbon synapses in sensory neurotransmission. Nat Rev Neurosci $11,812-822$.

M axeiner, S., Luo, F., Tan, A., Schmitz, F. and Südhof, T. C. (2016). How to make a synaptic ribbon: RIBEYE deletion abolishes ribbons in retinal synapses and disrupts neurotransmitter release. TheEM BO Journal 35, 1098-1114.

McMahon, H. T. and Boucrot, E. (2011). Molecular mechanism and physiological functions of clathrin-mediated endocytosis. Nat. Rev. Mol. Cell Biol. 12, 517-533.

M elcher, J. R., Guinan Jr, J. H., Knudson, I. M. and Kiang, N. Y. S. (1996). Generators of the brainstem auditory evoked potential in cat II. Correlating lesion sites with waveform changes. H earing research 93, 28-51.

M endoza Schulz, A., Jing, Z., Sánchez Caro, J. M., W etzel, F., Dresbach, T., Strenzke, N., Wichmann, C. and M oser, T. (2014). Bassoon-disruption slows vesicle replenishment and induces homeostatic plasticity at a CNS synapse. EM BO J. 33, 512-527. 
Merchan-Perez, A . and Liberman, M . C. (1996). Ultrastructural differences among afferent synapses on cochlear hair cells: correlations with spontaneous discharge rate. J. Comp. Neurol 371, 208-221.

M eyer, A. C., Frank, T., Khimich, D., H och, G., Riedel, D., Chapochnikov, N. M ., Yarin, Y. M ., Harke, B., H ell, S. W ., Egner, A., et al. (2009). Tuning of synapse number, structure and function in the cochlea. Nat Neurosci 12, 444-453.

M eyerholz, A., Hinrichsen, L., Groos, S., Esk, P.-C., Brandes, G. and Ungewickell, E. J. (2005). Effect of clathrin assembly lymphoid myeloid leukemia protein depletion on clathrin coat formation. Traffic 6, 1225-1234.

Michalski, N., Goutman, J. D., A uclair, S. M ., M onvel, J. B. de, Tertrais, M ., Emptoz, A., Parrin, A., N ouaille, S., Guillon, M., Sachse, M ., et al. (2017). Otoferlin acts as a Ca2+ sensor for vesicle fusion and vesicle pool replenishment at auditory hair cell ribbon synapses. elife Sciences 6, e31013.

Mikaelian, D. and Ruben, R. J. (1965). Development of Hearing in the Normal Cba-J M ouse: Correlation of Physiological Observations with Behavioral Responses and with Cochlear Anatomy. Acta Oto-laryngologica 59, 451-461.

Miller, S. E., Sahlender, D. A., Graham, S. C., Höning, S., Robinson, M. S., Peden, A. A. and Owen, D. J. (2011). The molecular basis for the endocytosis of small R-SNAREs by the clathrin adaptor CALM . Cell 147, 1118-1131.

M iller, S. E., M athiasen, S., Bright, N . A., Pierre, F., Kelly, B. T., Kladt, N., Schauss, A., M errifield, C. J., Stamou, D., Höning, S., et al. (2015). CALM regulates clathrin-coated vesicle size and maturation by directly sensing and driving membrane curvature. Dev. Cell 33, 163-175.

M ilosevic, I. (2018). Revisiting the Role of Clathrin-M ediated Endoytosis in Synaptic V esicle Recycling. Front Cell N eurosci $12,27$.

Milosevic, I., Giovedi, S., Lou, X., Raimondi, A., Collesi, C., Shen, H., Paradise, S., O’Toole, E., Ferguson, S., Cremona, O., et al. (2011). Recruitment of Endophilin to Clathrin-Coated Pit N ecks Is Required for Efficient V esicleU ncoating after Fission. Neuron 72, 587-601.

M im, C., Cui, H., Gawronski-Salerno, J. A., Frost, A., Lyman, E., Voth, G. A. and Unger, V. M. (2012). Structural basis of membrane bending by the N-BAR protein endophilin. Cell 149, 137-145.

M ohrmann, R., de W it, H., Verhage, M., N eher, E. and Sørensen, J. B. (2010). Fast vesicle fusion in living cells requires at least three SN ARE complexes. Science 330, 502-505.

Morales, M., Colicos, M. A. and Goda, Y. (2000). Actin-dependent regulation of neurotransmitter release at central synapses. Neuron 27, 539-550.

M organ, J. R., Prasad, K., H ao, W ., Augustine, G. J and Lafer, E. M . (2000). A Conserved Clathrin Assembly M otif Essential for Synaptic V esicle Endocytosis. J. N eurosci. 20, 8667-8676.

M orris, S. A., Schröder, S., Plessmann, U., W eber, K. and U ngewickell, E. (1993). Clathrin assembly protein A P180: primary structure, domain organization and identification of a clathrin binding site. EM BO J. 12, 667-675.

M oser, T. and Beutner, D. (2000). Kinetics of exocytosis and endocytosis at the cochlear inner hair cell afferent synapse of the mouse. Proc Natl Acad Sci U SA 97, 883-888.

M oser, T. and Vogl, C. (2016). N ew insights into cochlear sound encoding. F1000Research 5, 2081.

M oshkanbaryans, L., Chan, L.-S. and Graham, M. E. (2014). The Biochemical Properties and Functions of CALM and AP180 in Clathrin M ediated Endocytosis. M embranes (Basel) 4, 388-413.

M oshkanbaryans, L., Xue, J., W ark, J. R., Robinson, P. J. and Graham, M . E. (2016). A N ovel Sequence in A P180 and CA LM Promotes Efficient Clathrin Binding and Assembly. PLoS ONE 11, e0162050.

M urdoch, J. D., Rostosky, C. M., Gowrisankaran, S., Arora, A. S., Soukup, S.-F., Vidal, R., Capece, V., Freytag, S., Fischer, A ., Verstreken, P., et al. (2016). Endophilin-A Deficiency Induces the Foxo3a-Fbxo32 Network in the Brain and Causes Dysregulation of A utophagy and the U biquitin-Proteasome System. Cell Reports 17, 1071-1086.

N eef, J., Jung, S., W ong, A. B., Reuter, K., Pangršič, T., Chakrabarti, R., Kugler, S., Lenz, C., N ouvian, R., Boumil, R. M., et al. (2014). M odes and Regulation of Endocytic M embrane Retrieval in M ouse Auditory Hair Cells. J. Neurosci. 34, 705-716.

N eef, J., U rban, N. T., O hn, T.-L., Frank, T., Jean, P., H ell, S. W ., Willig, K. I. and M oser, T. (2018). Q uantitative optical nanophysiology of $\mathrm{Ca} 2+$ signaling at inner hair cell active zones. Nature Communications 9, 290.

Neher, E. (1998). Vesicle pools and Ca2+ microdomains: new tools for understanding their roles in neurotransmitter release. Neuron 20, 389-399.

Neher, E. (2006). A comparison between exocytic control mechanisms in adrenal chromaffin cells and a glutamatergic synapse. Pflügers A rchiv - European Journal of Physiology 453, 261-268.

Neher, E. (2010). W hat is rate-limiting during sustained synaptic activity: vesicle supply or the availability of release sites. Front. Syn. Neurosci. 2, 10.

Neves, G., Gomis, A. and Lagnado, L. (2001). Calcium influx selects the fast mode of endocytosis in the synaptic terminal of retinal bipolar cells. Proc. Natl. A cad. Sci. U.S.A. 98, 15282-15287.

Nouvian, R. (2007). Temperature enhances exocytosis efficiency at the mouse inner hair cell ribbon synapse. The Journal of Physiology 584, 535-542. 
N ouvian, R., N eef, J., Bulankina, A. V., Reisinger, E., Pangršič, T., Frank, T., Sikorra, S., Brose, N., Binz, T. and M oser, T. (2011). Exocytosis at the hair cell ribbon synapse apparently operates without neuronal SNARE proteins. Nat Neurosci 14, 411-413.

Oheim, M., Kirchhoff, F. and Stühmer, W. (2006). Calcium microdomains in regulated exocytosis. Cell Calcium 40, 423439.

Ohn, T.-L., Rutherford, M. A., Jing, Z., J ung, S., Duque-A fonso, C. J., Hoch, G., Picher, M. M ., Scharinger, A ., Strenzke, N . and Moser, T. (2016). H air cells use active zones with different voltage dependence of Ca2+ influx to decompose sounds into complementary neural codes. PNAS 113, 201605737.

Padmanarayana, M., H ams, N., Speight, L. C., Petersson, E. J., M ehl, R. A. and Johnson, C. P. (2014). Characterization of the lipid binding properties of Otoferlin reveals specific interactions between $\mathrm{PI}(4,5) \mathrm{P} 2$ and the $\mathrm{C} 2 \mathrm{C}$ and $\mathrm{C} 2 \mathrm{~F}$ domains. Biochemistry 53, 5023-5033.

Paillart, C., Li, J., M atthews, G. and Sterling, P. (2003). Endocytosis and vesicle recycling at a ribbon synapse. J. Neurosci. 23, 4092-4099.

Pangršič, T. and Vogl, C. (2018). Balancing presynaptic release and endocytic membrane retrieval at hair cell ribbon synapses. FEBS Lett.

Pangršič, T., Lasarow, L., Reuter, K., Takago, H., Schwander, M., Riedel, D., Frank, T., Tarantino, L. M., Bailey, J. S., Strenzke, N ., et al. (2010). Hearing requires otoferlin-dependent efficient replenishment of synaptic vesicles in hair cells. Nat. Neurosci. 13, 869-876.

Pangršič, T., Reisinger, E. and Moser, T. (2012). Otoferlin: a multi-C-2 domain protein essential for hearing. Trends Neurosci 35, 671-680.

Parsons, T. D., Lenzi, D., Almers, W. and Roberts, W. M. (1994). Calcium-triggered exocytosis and endocytosis in an isolated presynaptic cell: capacitance measurements in saccular hair cells. Neuron 13, 875-883.

Pechstein, A., Gerth, F., Milosevic, I., Jäpel, M., Eichhorn-Grünig, M., Vorontsova, O., Bacetic, J., Maritzen, T., Shupliakov, O., Freund, C., et al. (2015). Vesicle uncoating regulated by SH 3-SH3 domain-mediated complex formation between endophilin and intersectin at synapses. EM BO reports 16, 232-239.

Perera, R. M., Zoncu, R., Lucast, L., Camilli, P. D. and Toomre, D. (2006). Two synaptojanin 1 isoforms are recruited to clathrin-coated pits at different stages. PNAS 103, 19332-19337.

Picher, M. M., Oprişoreanu, A.-M ., Jung, S., M ichel, K., Schoch, S. and M oser, T. (2017). Rab Interacting M olecules 2 and 3 Directly Interact with the Pore-Forming CaV1.3 Ca2+ Channel Subunit and Promote Its Membrane Expression. Front Cell Neurosci 11.

Platzer, J., Engel, J., Schrott-Fischer, A., Stephan, K., Bova, S., Chen, H., Zheng, H. and Striessnig, J. (2000). Congenital deafness and sinoatrial node dysfunction in mice lacking class D L-type Ca2+channels. Cell 102, 89-97.

Puchkov, D. and H aucke, V. (2013). Greasing the synaptic vesicle cycle by membranelipids. Trends in Cell Biology 23, 493503.

Ramakrishnan, N. A., Drescher, M. J . and Drescher, D. G. (2009). Direct interaction of otoferlin with syntaxin 1A, SNAP25, and the L-type voltage-gated calcium channel Cav1.3. J. Biol. Chem 284, 1364-1372.

Regus-Leidig, H., Ott, C., Löhner, M., A torf, J., Fuchs, M., Sedmak, T., Kremers, J., Fejtová, A., Gundelfinger, E. D. and Brandstätter, J. H. (2013). I dentification and Immunocytochemical Characterization of Piccolino, a N ovel Piccolo Splice V ariant Selectively Expressed at Sensory Ribbon Synapses of the Eye and Ear. PLoS ONE 8, e70373.

Regus-Leidig, H., Fuchs, M., Löhner, M., Leist, S. R., Leal-Ortiz, S., Chiodo, V. A., H auswirth, W. W., Garner, C. C. and Brandstätter, J. H. (2014). In vivo knockdown of Piccolino disrupts presynaptic ribbon morphology in mouse photoreceptor synapses. Front Cell Neurosci 8, 259.

Reisinger, E., Bresee, C., N eef, J., N air, R., Reuter, K., Bulankina, A., N ouvian, R., Koch, M ., Bückers, J., Kastrup, L., et al. (2011). Probing the functional equivalence of otoferlin and synaptotagmin 1 in exocytosis. J. Neurosci. 31, 48864895.

Renard, H.-F., Simunovic, M ., Lemière, J., Boucrot, E., Garcia-Castillo, M . D., A rumugam, S., Chambon, V., Lamaze, C., Wunder, C., Kenworthy, A. K., et al. (2015). Endophilin-A2 functions in membrane scission in clathrinindependent endocytosis. Nature 517, 493-496.

Renden, R. and von Gersdorff, H. (2007). Synaptic Vesicle Endocytosis at a CNS Nerve Terminal: Faster Kinetics at Physiological Temperatures and Increased Endocytotic Capacity During Maturation. J Neurophysiol 98, 3349_ 3359.

Revelo, N. H., Kamin, D., Truckenbrodt, S., W ong, A . B., Reuter-Jessen, K., Reisinger, E., M oser, T. and Rizzoli, S. O. (2014). A new probe for super-resolution imaging of membranes elucidates trafficking pathways. J. Cell Biol. 205, 591606.

Ringstad, N., Nemoto, Y. and De Camilli, P. (1997). The SH 3p4/Sh3p8/SH 3p13 protein family: binding partners for synaptojanin and dynamin via a Grb2-likeSrc homology 3 domain. Proc. Natl. Acad. Sci. U.S.A. 94, 8569-8574. 
Ringstad, N., Gad, H., Löw, P., Paolo, G. D., Brodin, L., Shupliakov, O. and Camilli, P. D. (1999). Endophilin/SH 3p4 Is Required for the Transition from Early to Late Stages in Clathrin-M ediated Synaptic V esicle Endocytosis. Neuron 24, 143-154.

Rizzoli, S. O. and Betz, W. J. (2005). Synaptic vesicle pools. Nat. Rev. Neurosci. 6, 57-69.

Rizzoli, S. O. and Jahn, R. (2007). Kiss-and-run, Collapse and 'Readily Retrievable' Vesicles. Traffic 8, 1137-1144.

Rizzoli, S. O., Bethani, I., Zwilling, D., Wenzel, D., Siddiqui, T. J., Brandhorst, D. and Jahn, R. (2006). Evidence for early endosome-like fusion of recently endocytosed synaptic vesicles. Traffic 7, 1163-1176.

Roberts, W . M., Jacobs, R. A . and Hudspeth, A. J. (1990). Colocalization of ion channels involved in frequency selectivity and synaptic transmission at presynaptic active zones of hair cells. J Neurosci 10, 3664-3684.

Rosenmund, C. and Stevens, C. F. (1996). Definition of the readily releasable pool of vesicles at hippocampal synapses. Neuron 16, 1197-1207.

Rothman, J. E. and Schmid, S. L. (1986). Enzymatic recycling of clathrin from coated vesicles. Cell 46, 5-9.

Roux, I., Safieddine, S., N ouvian, R., Grati, M ., Simmler, M .-C., Bahloul, A ., Perfettini, I., Le Gall, M ., Rostaing, P., H amard, G., et al. (2006). Otoferlin, defective in a human deafness form, is essential for exocytosis at the auditory ribbon synapse. Cell 127, 277-289.

Roux, I., H osie, S., Johnson, S. L., Bahloul, A., Cayet, N., N ouaille, S., Kros, C. J., Petit, C. and Safieddine, S. (2009). M yosin $\mathrm{VI}$ is required for the proper maturation and function of inner hair cell ribbon synapses. Hum. M ol. Genet. 18, 4615-4628.

Rutherford, M. A . and Moser, T. (2016). The Ribbon Synapse Between Type I Spiral Ganglion N eurons and Inner Hair Cells. In The Primary A uditory N eurons of the M ammalian Cochlea (ed. Dabdoub, A.), Fritzsch, B.), Popper, A. N.), and Fay, R. R.), pp. 117-156. Springer N ew York.

Rutherford, M. A. and Roberts, W. M. (2006). Frequency selectivity of synaptic exocytosis in frog saccular hair cells. Proc Natl A cad Sci U SA 103, 2898.

Safieddine, S. and W enthold, R. J. (1999). SN ARE complex at the ribbon synapses of cochlear hair cells: analysis of synaptic vesicle and synaptic membrane-associated proteins. European Journal of Neuroscience 11, 803-812.

Safieddine, S., El-A mraoui, A . and Petit, C. (2012). The auditory hair cell ribbon synapse: from assembly to function. A nnu Rev Neurosci 35, 509-528.

Saheki, Y . and Camilli, P. D. (2012). Synaptic Vesicle Endocytosis. Cold Spring H arb Perspect Biol 4,.

Sakaba, T. and N eher, E. (2003). Involvement of A ctin Polymerization in V esicle Recruitment at the Calyx of H eld Synapse. The Journal of Neuroscience 23, 837-846.

Sanchez del Rio, M. and Pareschi, G. (2001). Global optimization and reflectivity data fitting for x-ray multilayer mirrors by mean s of genetic algorithms. SPIE Proceedings 88-96.

Sato, K., Ernstrom, G. G., W atanabe, S., W eimer, R. M ., Chen, C.-H ., Sato, M ., Siddiqui, A., Jorgensen, E. M . and Grant, B. D. (2009). Differential requirements for clathrin in receptor-mediated endocytosis and maintenance of synaptic vesicle pools. Proc. Natl. A cad. Sci. U.S.A. 106, 1139-1144.

Schikorski, T. and Stevens, C. F. (2001). Morphological correlates of functionally defined synaptic vesicle populations. Nature Neuroscience 4, 391-395.

Schmitz, F., Königstorfer, A. and Südhof, T. C. (2000). RIBEYE, a component of synaptic ribbons: a protein's journey through evolution provides insight into synaptic ribbon function. Neuron 28, 857-872.

Schnee, M . E., Lawton, D. M ., Furness, D. N ., Benke, T. A . and Ricci, A. J. (2005). A uditory hair cell-afferent fiber synapses are specialized to operate at their best frequencies. Neuron 47, 243-254.

Schneggenburger, R., Meyer, A. C. and Neher, E. (1999). Released Fraction and Total Size of a Pool of Immediately A vailable Transmitter Q uanta at a Calyx Synapse. Neuron 23, 399-409.

Schuske, K. R., Richmond, J. E., M atthies, D. S., Davis, W. S., Runz, S., Rube, D. A., van der Bliek, A. M . and Jorgensen, E. M . (2003). Endophilin Is Required for Synaptic VesicleEndocytosis by Localizing Synaptojanin. Neuron 40, 749_ 762.

Schwarz, K., N atarajan, S., Kassas, N ., V itale, N . and Schmitz, F. (2011). The Synaptic Ribbon Is a Site of Phosphatidic A cid Generation in Ribbon Synapses. J. Neurosci. 31, 15996-16011.

Sendin, G., Bulankina, A. V., Riedel, D. and Moser, T. (2007). Maturation of ribbon synapses in hair cells is driven by thyroid hormone. J Neurosci 27, 3163-3173.

Sheets, L., Trapani, J. G., M o, W ., Obholzer, N . and N icolson, T. (2011). Ribeye is required for presynaptic CaV 1.3a channel localization and afferent innervation of sensory hair cells. Development 138, 1309-1319.

Shnerson, A. and Pujol, R. (1981). Age-related changes in the C57BL/6J mouse cochlea. I. Physiological findings. Developmental Brain Research 2, 65-75.

Siegel, J. H. and Brownell, W. E. (1986). Synaptic and Golgi membrane recycling in cochlear hair cells. J. Neurocytol 15, 311-328. 
Siksou, L., Rostaing, P., Lechaire, J.-P., Boudier, T., O htsuka, T., Fejtová, A., Kao, H .-T., Greengard, P., Gundelfinger, E. D., Triller, A., et al. (2007). Three-dimensional architecture of presynaptic terminal cytomatrix. J N eurosci 27, 68686877.

Simunovic, M ., M anneville, J.-B., Renard, H .-F., Evergren, E., Raghunathan, K., Bhatia, D., Kenworthy, A. K., V oth, G. A., Prost, J., M cM ahon, H. T., et al. (2017). Friction M ediates Scission of Tubular M embranes Scaffolded by BAR Proteins. Cell 170, 172-184.el1.

Sinha, R., A hmed, S., Jahn, R. and Klingauf, J. (2011). Two synaptobrevin molecules are sufficient for vesicle fusion in central nervous system synapses. Proc. Natl. Acad. Sci. U S.A. 108, 14318-14323.

Sjostrand, F. S. (1958). Ultrastructure of retinal rod synapses of the guinea pig eye as revealed by three-dimensional reconstructions from serial sections. J. Ultrastruct. Res. 2, 122-170.

Slepecky, N. and Chamberlain, S. C. (1985). Immunoelectron microscopic and immunofluorescent localization of cytoskeletal and muscle-like contractile proteins in inner ear sensory hair cells. Hear. Res. 20, 245-260.

Smith, C. A. and Sjöstrand, F. S. (1961). A synaptic structure in the hair cells of the guinea pig cochlea. Journal of Ultrastructure Research 5, 184-192.

Snellman, J., M ehta, B., Babai, N ., Bartoletti, T. M ., A kmentin, W ., Francis, A., M atthews, G., Thoreson, W . and Zenisek, D. (2011). A cute destruction of the synaptic ribbon reveals a role for the ribbon in vesicle priming. $N$ at N eurosci 14, 1135-1141.

Sobkowicz, H. M., Rose, J. E., Scott, G. E. and Slapnick, S. M . (1982). Ribbon synapses in the developing intact and cultured organ of Corti in the mouse. J. Neurosci. 2, 942-957.

Sobkowicz, H. M., Rose, J. E., Scott, G. L. and Levenick, C. V. (1986). Distribution of synaptic ribbons in the developing organ of Corti. J. Neurocytol 15, 693-714.

Soda, K., Balkin, D. M ., Ferguson, S. M ., Paradise, S., M ilosevic, I., Giovedi, S., Volpicelli-Daley, L., Tian, X., W u, Y., M a, H., et al. (2012). Role of dynamin, synaptojanin, and endophilin in podocyte foot processes. J. Clin. Invest. 122, $4401-4411$.

Söllner, T., W hiteheart, S. W ., Brunner, M., Erdjument-Bromage, H., Geromanos, S., Tempst, P. and Rothman, J. E. (1993). SNAP receptors implicated in vesicle targeting and fusion. , Published online: $25 \mathrm{M}$ arch 1993; । doi:10.1038/362318a0 362, 318-324.

Soukup, S.-F., Kuenen, S., V anhauwaert, R., M anetsberger, J., Hernández-Díaz, S., Swerts, J., Schoovaerts, N., Vilain, S., Gounko, N. V., Vints, K., et al. (2016). A LRRK2-Dependent EndophilinA Phosphoswitch Is Critical for $M$ acroautophagy at Presynaptic Terminals. Neuron 92, 829-844.

Soykan, T., Kaempf, N., Sakaba, T., Vollweiter, D., Goerdeler, F., Puchkov, D., Kononenko, N. L. and H aucke, V. (2017). Synaptic Vesicle Endocytosis Occurs on Multiple Timescales and Is Mediated by Formin-Dependent Actin Assembly. Neuron 93, 854-866.e4.

Strenzke, N ., Chanda, S., Kopp-Scheinpflug, C., Khimich, D ., Reim, K., Bulankina, A. V., N eef, A., W olf, F., Brose, N ., XuFriedman, M. A., et al. (2009). Complexin-I Is Required for High-Fidelity Transmission at the Endbulb of Held Auditory Synapse. J. N eurosci. 29, 7991-8004.

Strenzke, N ., Chakrabarti, R., Al-M oyed, H ., M üller, A., H och, G., Pangršič, T., Y amanbaeva, G., Lenz, C., Pan, K.-T., A uge, E., et al. (2016). Hair cell synaptic dysfunction, auditory fatigue and thermal sensitivity in otoferlin Ile515Thr mutants. EM BO J. 35, 2519-2535.

Südhof, T. C. (2012). The presynaptic active zone. Neuron 75, 11-25.

Südhof, T. C. and Rothman, J. E. (2009). M embrane Fusion: Grappling with SN ARE and SM Proteins. Science 323, 474477.

Sundborger, A., Soderblom, C., V orontsova, O., Evergren, E., Hinshaw, J. E. and Shupliakov, O. (2011). An endophilindynamin complex promotes budding of clathrin-coated vesicles during synaptic vesicle recycling. J Cell Sci 124, 133-143.

Sutton, R. B., Fasshauer, D., Jahn, R. and Brunger, A. T. (1998). Crystal structure of a SN ARE complex involved in synaptic exocytosis at 2.4 A resolution. Nature 395, 347-353.

Takamori, S., H olt, M ., Stenius, K., Lemke, E. A., Grønborg, M ., Riedel, D., U rlaub, H ., Schenck, S., Brügger, B. and Ringler, P. (2006). M olecular A natomy of a Trafficking Organelle. Cell 127, 831-846.

Takei, K. (1996). The synaptic vesicle cycle: a single vesicle budding step involving clathrin and dynamin. The Journal of Cell Biology 133, 1237-1250.

tom Dieck, S., Sanmartí-Vila, L., Langnaese, K., Richter, K., Kindler, S., Soyke, A., W ex, H., Smalla, K. H., Kämpf, U., Fränzer, J. T., et al. (1998). Bassoon, a novel zinc-finger CAG/glutamine repeat protein selectively localized at the active zone of presynaptic nerve terminals. J Cell Biol 142, 499-509.

tom Dieck, S., Altrock, W. D., Kessels, M. M ., Qualmann, B., Regus, H., Brauner, D., Fejtová, A., Bracko, O., Gundelfinger, E. D. and Brandstätter, J. H. (2005). Molecular dissection of the photoreceptor ribbon synapse: physical interaction of Bassoon and RIBEYE is essential for the assembly of the ribbon complex. J Cell Biol 168, 825-836. 
Trapani, J. G., Obholzer, N., M o, W., Brockerhoff, S. E. and Nicolson, T. (2009). synaptojanin1 Is Required for Temporal Fidelity of Synaptic Transmission in Hair Cells. PLoS Genetics 5, el000480.

Trifaró, J. M., Rodríguez del Castillo, A. and Vitale, M. L. (1992). Dynamic changes in chromaffin cell cytoskeleton as prelude to exocytosis. M ol. Neurobiol. 6, 339-358.

Truckenbrodt, S., Viplav, A., Jähne, S., Vogts, A., Denker, A., Wildhagen, H., Fornasiero, E. F. and Rizzoli, S. O. (2018). N ewly produced synaptic vesicle proteins are preferentially used in synaptic transmission. EM BO J. 37.

van den Bogaart, G., H olt, M. G., Bunt, G., Riedel, D., W outers, F. S. and Jahn, R. (2010). One SN A RE complex is sufficient for membrane fusion. Nat. Struct. M ol. Biol. 17, 358-364.

Vehlow, A., Soong, D., Vizcay-Barrena, G., Bodo, C., Law, A.-L., Perera, U. and Krause, M. (2013). Endophilin, Lamellipodin, and M ena cooperate to regulate F-actin-dependent EGF-receptor endocytosis. EM BO J. 32, 27222734.

Verhage, M. and Sørensen, J. B. (2008). V esicle Docking in Regulated Exocytosis. Traffic 9, 1414-1424.

Verstreken, P., Kjaerulff, O., Lloyd, T. E., Atkinson, R., Zhou, Y., M einertzhagen, I. A. and Bellen, H. J. (2002). Endophilin M utations Block Clathrin-M ediated Endocytosis but N ot N eurotransmitter Release. Cell 109, 101-112.

Verstreken, P., Koh, T.-W., Schulze, K. L., Zhai, R. G., Hiesinger, P. R., Zhou, Y., M ehta, S. Q ., Cao, Y., Roos, J. and Bellen, H. J. (2003). Synaptojanin Is Recruited by Endophilin to Promote Synaptic Vesicle Uncoating. Neuron 40, 733748.

Vincent, P. F., Bouleau, Y., Petit, C. and Dulon, D. (2015). A synaptic F-actin network controls otoferlin-dependent exocytosis in auditory inner hair cells. eLife 4,.

Vitale, M. L., Seward, E. P. and Trifaró, J. M. (1995). Chromaffin cell cortical actin network dynamics control the size of the release ready vesicle pool and the initial rate of exocytosis. Neuron 14, 353-363.

Vogl, C., Cooper, B. H., N eef, J., W ojcik, S. M., Reim, K., Reisinger, E., Brose, N., Rhee, J.-S., M oser, T. and Wichmann, C. (2015). Unconventional molecular regulation of synaptic vesiclereplenishment in cochlear inner hair cells. J. Cell. Sci. 128, 638-644.

Vogl, C., Panou, I., Yamanbaeva, G., Wichmann, C., M angosing, S. J., Vilardi, F., Indzhykulian, A. A., Pangršič, T., Santarelli, R., Rodriguez-Ballesteros, M ., et al. (2016). Tryptophan-rich basic protein (WRB) mediates insertion of the tail-anchored protein otoferlin and is required for hair cell exocytosis and hearing. EM BO J. e201593565.

Vogl, C., Butola, T., Haag, N., H ausrat, T. J., Leitner, M. G., M outschen, M., Lefèbvre, P. P., Speckmann, C., Garrett, L., Becker, L., et al. (2017). The BEACH protein LRBA is required for hair bundle maintenance in cochlear hair cells and for hearing. EM BO Rep. 18, 2015-2029.

Wagh, D. A., Rasse, T. M., Asan, E., H ofbauer, A., Schwenkert, I., Dürrbeck, H., Buchner, S., Dabauvalle, M . C., Schmidt, M., Qin, G., et al. (2006). Bruchpilot, a protein with homology to ELKS/CAST, is required for structural integrity and function of synaptic active zones in Drosophila. Neuron 49, 833-844.

Wahl, S., Katiyar, R. and Schmitz, F. (2013). A Local, Periactive Zone Endocytic Machinery at Photoreceptor Synapses in Close Vicinity to Synaptic Ribbons. J. Neurosci. 33, 10278-10300.

Watanabe, S. and Boucrot, E. (2017). Fast and ultrafast endocytosis. Curr. Opin. Cell Biol. 47, 64-71.

Watanabe, S., Rost, B. R., Camacho-Pérez, M., Davis, M. W., Söhl-Kielczynski, B., Rosenmund, C. and Jorgensen, E. M. (2013). U Itrafast endocytosis at mouse hippocampal synapses. Nature 504, 242-247.

W atanabe, S., Trimbuch, T., Camacho-Pérez, M., Rost, B. R., Brokowski, B., Söhl-Kielczynski, B., Felies, A., Davis, M. W ., Rosenmund, C. and Jorgensen, E. M. (2014). Clathrin regenerates synaptic vesicles from endosomes. Nature 515, 228-233.

Watanabe, S., Mamer, L. E., Raychaudhuri, S., Luvsanjav, D., Eisen, J., Trimbuch, T., Söhl-Kielczynski, B., Fenske, P., Milosevic, I., Rosenmund, C., et al. (2018). Synaptojanin and Endophilin Mediate Neck Formation during Ultrafast Endocytosis. Neuron 98, 1184-1197.e6.

W eiler, S., Krinner, S., Wong, A. B., Moser, T. and Pangršič, T. (2014). ATP Hydrolysis Is Critically Required for Function of CaV 1.3 Channels in Cochlear Inner H air Cells via Fueling Ca2+Clearance. J Neurosci 34, 6843-6848.

Wichmann, C. and M oser, T. (2015). Relating structure and function of inner hair cell ribbon synapses. Cell Tissue Res.

Wiederhold, K. and Fasshauer, D. (2009). Is A ssembly of the SN ARE Complex Enough to Fuel M embrane Fusion? J. Biol. Chem. 284, 13143-13152.

Wilhelm, B. G., M andad, S., Truckenbrodt, S., Kröhnert, K., Schäfer, C., Rammner, B., Koo, S. J., Claßen, G. A., Krauss, M .,

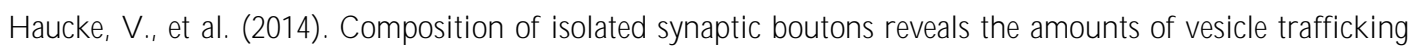
proteins. Science 344, 1023-1028.

Willox, A. K., Sahraoui, Y. M. E. and Royle, S. J. (2014). Non-specificity of Pitstop 2 in clathrin-mediated endocytosis. Biol Open 3, 326-331.

Wong, A. B., Rutherford, M. A., Gabrielaitis, M., Pangršič, T., Gottfert, F., Frank, T., M ichanski, S., Hell, S., W olf, F., W ichmann, C., et al. (2014). Developmental refinement of hair cell synapses tightens the coupling of Ca2+ influx to exocytosis. Embo J 33, 247-264. 
Wu, W . and Wu, L.-G. (2007). Rapid bulk endocytosis and its kinetics of fission pore closure at a central synapse. Proc. Natl. Acad. Sci. U.S.A. 104, 10234-10239.

Wu, X.-S., M cN eil, B. D., Xu, J., Fan, J., Xue, L., M elicoff, E., A dachi, R., Bai, L. and W u, L.-G. (2009). Ca2+ and calmodulin initiate all forms of endocytosis during depolarization at a nerve terminal. Nat Neurosci 12, 1003-1010.

Wu, Y., O’Toole, E. T., Girard, M., Ritter, B., Messa, M., Liu, X., McPherson, P. S., Ferguson, S. M. and De Camilli, P. (2014). A dynamin 1-, dynamin 3- and clathrin-independent pathway of synaptic vesicle recycling mediated by bulk endocytosis. Elife 3, e01621.

Xing, Y., Böcking, T., W olf, M ., Grigorieff, N., Kirchhausen, T. and Harrison, S. C. (2010). Structure of clathrin coat with bound H sc70 and auxilin: mechanism of Hsc70-facilitated disassembly. The EM BO Journal 29, 655-665.

Yang, Y., W ei, M ., Xiong, Y., Du, X., Zhu, S., Yang, L., Zhang, C. and Liu, J.-J. (2015). Endophilin A 1 regulates dendritic spine morphogenesis and stability through interaction with p140Cap. Cell Res. 25, 496-516.

Zampini, V., Johnson, S. L., Franz, C., Knipper, M., H olley, M. C., M agistretti, J., M asetto, S. and M arcotti, W . (2013). Burst activity and ultrafast activation kinetics of $\mathrm{CaV} 1.3 \mathrm{Ca2}+$ channels support presynaptic activity in adult gerbil hair cell ribbon synapses. J Physiol 591, 3811-3820.

Zhai, R. G. and Bellen, H. J. (2004). The Architecture of the A ctive Zone in the Presynaptic Nerve Terminal. Physiology 19, 262-270.

Zhang, B., Koh, Y. H., Beckstead, R. B., Budnik, V., Ganetzky, B. and Bellen, H. J. (1998). Synaptic Vesicle Sizeand N umber Are Regulated by a Clathrin Adaptor Protein Required for Endocytosis. Neuron 21, 1465-1475.

Zhou, Z., M isler, S. and Chow, R. H. (1996). Rapid fluctuations in transmitter release from single vesicles in bovine adrenal chromaffin cells. Biophys. J. 70, 1543-1552.

Zhuo, Y., Ilangovan, U., Schirf, V., Demeler, B., Sousa, R., Hinck, A. P. and Lafer, E. M. (2010). Dynamic Interactions between Clathrin and Locally Structured Elements in a Disordered Protein M ediate Clathrin Lattice Assembly. Journal of M olecular Biology 404, 274-290. 
References 


\section{APPENDIX}

\section{a) AdDitional Material FOR THE ENDOPHILIN-A StUdY}

For Appendix Tables S1-S8 (related to figures created by the coauthors) please see the Supplemental Information for Kroll et al., 2019.

Appendix Table S9. Statistics for quantifications of structures in the ribbon proximity in IHCs from p15p17 Wt and endophilin-deficient mice (related to Fig. 2-7).

\begin{tabular}{|c|c|c|c|c|}
\hline N cytosolic SVs & \multicolumn{2}{|c|}{ Kruskal-W allis } & KWS $=130$ & $p<0.0001$ \\
\hline Post-hoc Dunn's & Wt & 1-SKO & 1/2-DKO & 1/3-DKO \\
\hline $\mathrm{Wt}$ & & $<0.0001$ & 0.0008 & 0.6240 \\
\hline $\mathrm{N}$ of ELVs & \multicolumn{2}{|c|}{ Kruskal-Wallis } & KWS $=70.17$ & $p<0.0001$ \\
\hline Post-hoc Dunn's & Wt & 1-SKO & 1/2-DKO & 1/3-DKO \\
\hline Wt & & $>0.9999$ & $<0.0001$ & 0.0027 \\
\hline $\begin{array}{l}\text { All coated } \\
\text { structures }\end{array}$ & \multicolumn{2}{|c|}{ Kruskal-Wallis } & KWS $=66.1$ & $p<0.0001$ \\
\hline Post-hoc Dunn's & Wt & 1-SKO & 1/2-DKO & 1/3-DKO \\
\hline Wt & & 0.0252 & $<0.0001$ & 0.0005 \\
\hline Coated vesicles & \multicolumn{2}{|c|}{ Kruskal-Wallis } & KWS $=27.13$ & $p<0.0001$ \\
\hline Post-hoc Dunn's & Wt & 1-SKO & $1 / 2$-DKO & 1/3-DKO \\
\hline Wt & & $<0.0001$ & 0.2076 & $>0.9999$ \\
\hline Coated vacuoles & \multicolumn{2}{|c|}{ Kruskal-Wallis } & $K W S=33$ & $p<0.0001$ \\
\hline Post-hoc Dunn's & $W t$ & 1-SKO & 1/2-DKO & 1/3-DKO \\
\hline $\mathrm{Wt}$ & & $>0.9999$ & $<0.0001$ & 0.0628 \\
\hline Coated pits & \multicolumn{2}{|c|}{ Kruskal-Wallis } & KWS $=79.67$ & $p<0.0001$ \\
\hline Post-hoc Dunn's & Wt & 1-SKO & 1/2-DKO & 1/3-DKO \\
\hline Wt & & $>0.9999$ & $<0.0001$ & 0.0007 \\
\hline C. pits at ELVs & \multicolumn{2}{|c|}{ Kruskal-Wallis } & KWS $=6.503$ & $p=0.0895$ \\
\hline
\end{tabular}

Appendix Table S10. Statistics for quantifications of structures in the ribbon proximity with comparisons of all genotypes with each other in IHCs from p15-p17 Wt and endophilin-deficient mice (related to Fig. 2-7).

\begin{tabular}{|c|c|c|c|c|}
\hline N cytosolic SVs & \multicolumn{2}{|c|}{ Kruskal-Wallis } & KWS $=130$ & $\mathrm{p}<0.0001$ \\
\hline Post-hoc Dunn's & Wt & 1-SKO & $1 / 2-D K O$ & $1 / 3-D K O$ \\
\hline Wt & & $<0.0001$ & 0.0016 & $>0.9999$ \\
\hline 1-SKO & & & $<0.0001$ & $<0.0001$ \\
\hline 1/2-DKO & \multicolumn{2}{|c|}{ Kruskal-Wallis } & KWS $=70.17$ & $\mathrm{p}<0.00001$ \\
\hline N of ELVs & Wt & 1-SKO & $1 / 2-D K 0$ & $1 / 3-D K 0$ \\
\hline Post-hoc Dunn's & & $>0.9999$ & $<0.0001$ & 0.0053 \\
\hline Wt & & & $<0.0001$ & 0.0026 \\
\hline 1-SKO & & & & $<0.0001$ \\
\hline 1/2-DKO & & &
\end{tabular}




\begin{tabular}{|c|c|c|c|c|}
\hline $\begin{array}{l}\text { All coated } \\
\text { structures }\end{array}$ & \multicolumn{2}{|c|}{ Kruskal-Wallis } & $K W S=66.1$ & $p<0.0001$ \\
\hline Post-hoc Dunn's & Wt & $1-S K O$ & 1/2-DKO & 1/3-DKO \\
\hline Wt & & 0.0505 & $<0.0001$ & 0.0009 \\
\hline 1-SKO & & & $<0.0001$ & $>0.9999$ \\
\hline 1/2-DKO & & & & $<0.0001$ \\
\hline Coated vesicles & \multicolumn{2}{|c|}{ Kruskal-W allis } & $K W S=27.13$ & $p<0.0001$ \\
\hline Post-hoc Dunn's & Wt & $1-S K O$ & $1 / 2-\mathrm{DKO}$ & 1/3-DKO \\
\hline Wt & & 0.0002 & 0.4152 & $>0.9999$ \\
\hline 1-SKO & & & 0.0256 & $<0.0001$ \\
\hline 1/2-DKO & & & & 0.4251 \\
\hline Coated vacuoles & \multicolumn{2}{|c|}{ Kruskal-W allis } & KWS = 33 & $p<0,0001$ \\
\hline Post-hoc Dunn's & $\mathrm{Wt}$ & 1-SKO & 1/2-DKO & 1/3-DKO \\
\hline $\mathrm{Wt}$ & & $>0.9999$ & $<0.0001$ & 0.1256 \\
\hline 1-SKO & & & $<0.0001$ & 0.3183 \\
\hline 1/2-DKO & & & & 0.0053 \\
\hline Coated pits & \multicolumn{2}{|c|}{ Kruskal-Wallis } & $K W S=79.67$ & $p<0.0001$ \\
\hline Post-hoc Dunn's & $\mathrm{Wt}$ & 1-SKO & $1 / 2-\mathrm{DKO}$ & 1/3-DKO \\
\hline $\mathrm{Wt}$ & & $>0.9999$ & $<0.0001$ & 0.0014 \\
\hline 1-SKO & & & $<0.0001$ & $<0.0001$ \\
\hline 1/2-DKO & & & & 0.0004 \\
\hline C. pits at ELVs & \multicolumn{2}{|c|}{ Kruskal-Wallis } & KWS $=6.503$ & $p=0.0895$ \\
\hline
\end{tabular}

Appendix Table S11. Statistics for synaptic vesicles around the synaptic ribbon in IHCs from p15-p17 Wt and endophilin-deficient mice (related to Fig. 2-8).

\begin{tabular}{|c|c|c|c|c|}
\hline $\begin{array}{c}\mathrm{N} \text { of RA-SVs } \\
\text { (random sections) }\end{array}$ & \multicolumn{2}{|c|}{ one-way ANOVA } & $F(3,417)=38.4$ & $p<0.0001$ \\
\hline Post-hoc Tukey & Wt & 1-SKO & 1/2-DKO & 1/3-DKO \\
\hline Wt & & 0.2315 & 0.0001 & 0.0012 \\
\hline $\begin{array}{c}\mathrm{N} \text { of MP-SV s } \\
\text { (random sections) }\end{array}$ & \multicolumn{2}{|c|}{ one-way ANOVA } & $F(3,425)=17.6$ & $p<0.0001$ \\
\hline Post-hoc Tukey & Wt & 1-SKO & 1/2-DKO & 1/3-DKO \\
\hline Wt & & 0.1959 & $<0.0001$ & $<0.0001$ \\
\hline $\begin{array}{l}\text { Distal:proximal } \\
\text { (random sections) }\end{array}$ & \multicolumn{2}{|c|}{ Kruskal-Wallis } & KWS = 82.4 & $p<0.0001$ \\
\hline Post-hoc D unn's & Wt & 1-SKO & 1/2-DKO & 1/3-DKO \\
\hline Wt & & $>0.9999$ & $<0.0001$ & $<0.0001$ \\
\hline
\end{tabular}


Appendix Table S12. Statistics for quantifications of synaptic vesicles around the ribbon with comparisons of all genotypes with each other in IHCs from p15-p17 Wt and endophilin-deficient mice (related to Fig. 2-8).

\begin{tabular}{|c|c|c|c|c|}
\hline $\begin{array}{c}\mathrm{N} \text { of RA-SVs } \\
\text { (random sections) }\end{array}$ & \multicolumn{2}{|c|}{ one-way ANOVA } & $F(3,417)=38.4$ & $p<0.0001$ \\
\hline Post-hoc Tukey & Wt & 1-SKO & 1/2-DKO & 1/3-DKO \\
\hline Wt & & 0.3466 & $<0.0001$ & 0.0022 \\
\hline 1-SKO & & & $<0.0001$ & $<0.0001$ \\
\hline 1/2-DKO & & & & $<0.0001$ \\
\hline $\begin{array}{l}\text { RA-SV s distance } \\
\text { (random sections) }\end{array}$ & \multicolumn{2}{|c|}{ Kruskal-Wallis } & KWS $=15.3$ & $p=0.0015$ \\
\hline Post-hoc D unn's & Wt & $1-S K O$ & 1/2-DKO & 1/3-DKO \\
\hline Wt & & $>0.9999$ & 0.5220 & 0.0746 \\
\hline 1-SKO & & & 0.0548 & 0.0027 \\
\hline 1/2-DKO & & & & $>0.9999$ \\
\hline $\begin{array}{c}\mathrm{N} \text { of MP-SVs } \\
\text { (random sections) }\end{array}$ & \multicolumn{2}{|c|}{ one-way ANOVA } & $F(3,425)=17.6$ & $p<0.0001$ \\
\hline Post-hoc Tukey & $\mathrm{Wt}$ & 1-SKO & 1/2-DKO & 1/3-DKO \\
\hline Wt & & 0.1959 & $<0.0001$ & $<0.0001$ \\
\hline 1-SKO & & & $<0.0001$ & 0.0075 \\
\hline 1/2-DKO & & & & 0.0132 \\
\hline $\begin{array}{l}\text { MP-SV s distance } \\
\text { (random sections) }\end{array}$ & \multicolumn{2}{|c|}{ one-way A NOVA } & $F(3,423)=3.31$ & $p=0.02$ \\
\hline Post-hoc Tukey & $\mathrm{Wt}$ & 1-SKO & 1/2-DKO & 1/3-DKO \\
\hline Wt & & $>0.9999$ & 0.2293 & 0.8930 \\
\hline 1-SKO & & & 0.2334 & 0.8753 \\
\hline 1/2-DKO & & & & 0.0111 \\
\hline $\begin{array}{c}\text { apical-basal } \\
\text { (random sections) }\end{array}$ & \multicolumn{2}{|c|}{ Kruskal-Wallis } & KWS = 82.4 & $p<0.0001$ \\
\hline Post-hoc D unn's & Wt & 1-SKO & 1/2-DKO & 1/3-DKO \\
\hline Wt & & $>0.9999$ & $<0.0001$ & 0.0002 \\
\hline 1-SKO & & & $<0.0001$ & $<0.0001$ \\
\hline 1/2-DKO & & & & 0.0019 \\
\hline
\end{tabular}

Appendix Table S13. Statistics for quantifications of EM samples after stimulation in IHCs from p15-p16 Wt and endophilin-deficient mice (related to Fig. 2-9).

\begin{tabular}{|c|c|c|c|}
\hline N of RA-SVs & Mann-Whitney test & $\mathbf{U}=\mathbf{3 6 8}$ & $\mathrm{p}<0.0001$ \\
\hline N of MP-SVs & Mann-Whitney test & $\mathbf{U}=\mathbf{7 8 3}$ & $\mathrm{p}=0.0438$ \\
\hline cytosolic SVs & unpaired t test & $\mathbf{t}=\mathbf{4 . 6 7}$ & $\mathrm{p}<0.0001$ \\
\hline ELVs & Mann-Whitney test & $\mathbf{U}=\mathbf{2 6 4}$ & $\mathrm{p}<0.0001$ \\
\hline coated structures & Mann-Whitney test & $\mathbf{U}=\mathbf{3 1 2}$ & $\mathrm{p}<0.0001$ \\
\hline Coated vesicles & Mann-Whitney test & $\mathbf{U}=\mathbf{3 2 7}$ & $\mathrm{p}<0.0001$ \\
\hline Coated vacuoles & Mann-Whitney test & $\mathbf{U}=\mathbf{8 8 2}$ & $\mathbf{p}=\mathbf{0 . 3 7 0 7}$ \\
\hline Coated pits & Mann-Whitney test & $\mathbf{U}=\mathbf{7 6 6}$ & $\mathbf{p}=\mathbf{0 . 0 6 1 8}$ \\
\hline C. pits at ELVs & Mann-Whitney test & $\mathbf{U}=\mathbf{6 2 3}$ & $\mathrm{p}=0.001$ \\
\hline
\end{tabular}




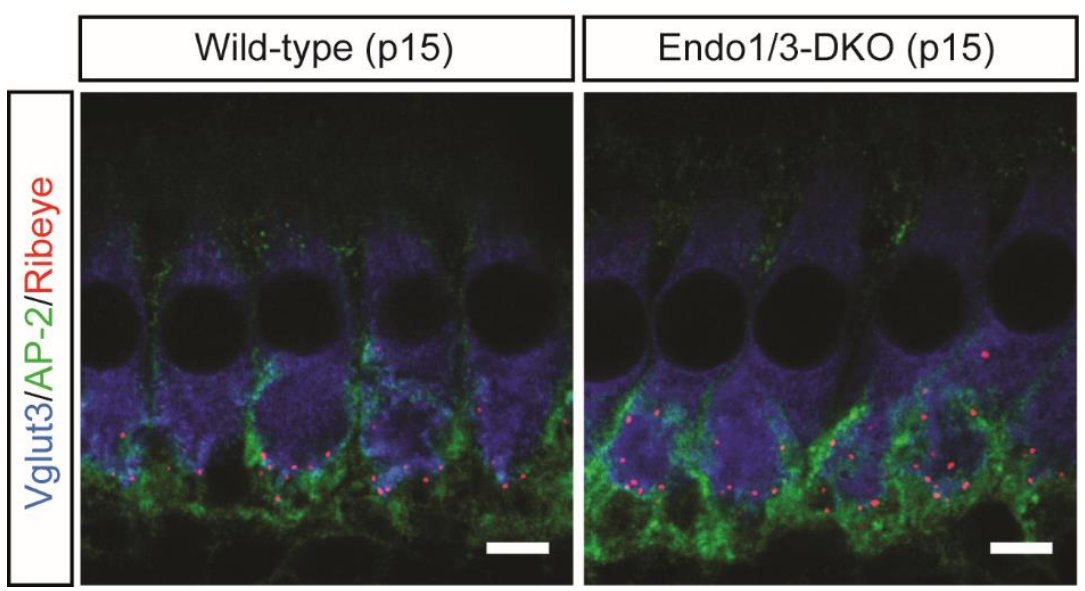

Appendix Fig. S1: AP-2 distribution appears unaltered in Endophilin1/3-DKO IHCs. Combined loss of endophilin $A 1$ and $A 3$ does not appear to majorly affect AP-2 expression in P15 IHCs or afferent auditory nerve fibers. All samples were processed in parallel and imaged with identical settings. Scale bar: $5 \mu \mathrm{m}$.

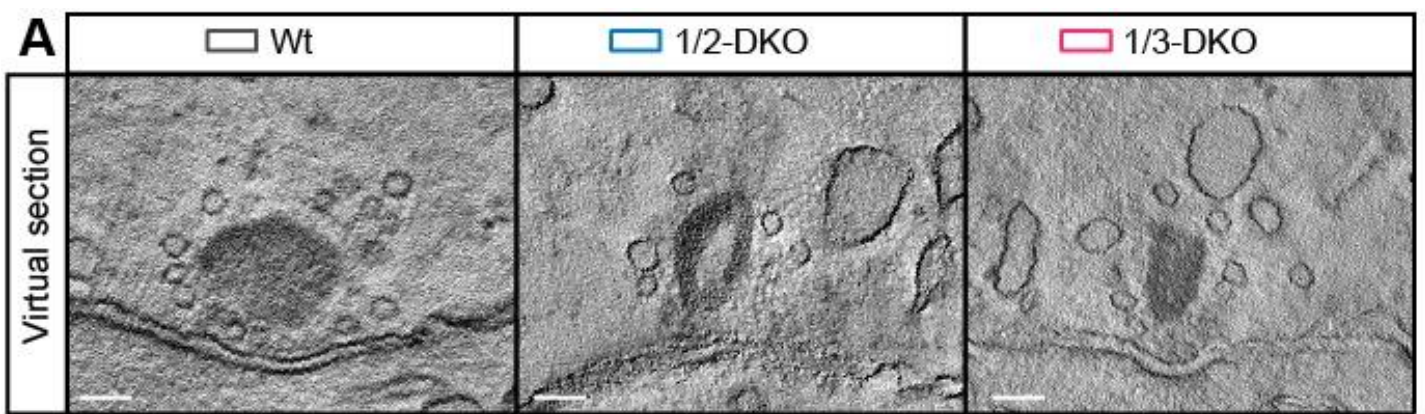

B

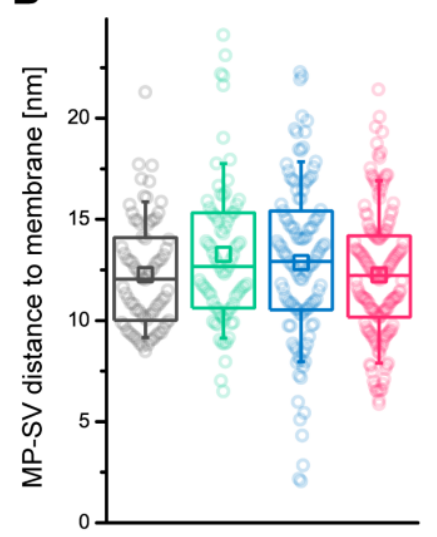

C

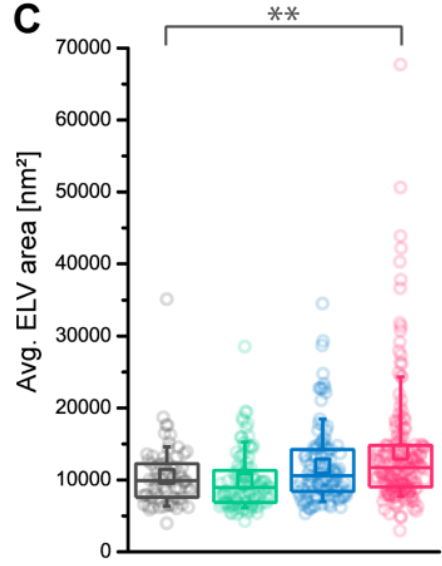

D

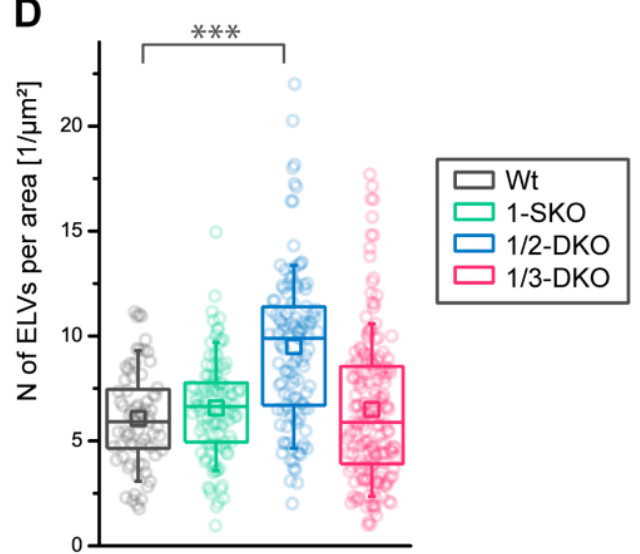

Appendix Fig. S2: Additional data from electron microscopy experiments. (A) Electron tomography virtual sections from Wt, 1/2-DKO and 1/3-DKO. Scale bar $100 \mathrm{~nm}$. (B) Quantification of distances between MP-SV and AZ membrane revealed no significant difference. Wt $\mathrm{n}=78$ sections from $\mathrm{N}=2$ animals; 1 -SKO $n=95, N=3 ; 1 / 2-D K O n=135, N=3 ; 1 / 3-D K O n=176, N=6$. one-way ANOVA followed by Tukey's post-hoc test. (C, D) The average area of ELVs was significantly increased in 1/3-DKOs (C), the number of ELVs per area of cytosol was higher in 1/2-DKOs compared to Wt, both being valid for all ELVs within $1 \mu \mathrm{m}$ from the ribbon. Wt $n=72 / N=2 ; 1-S K O n=106 / N=3 ; 1 / 2-D K O n=129 / N=3 ; 1 / 3-D K O n=172 / N=6$; Kruskal-Wallis test followed by Dunn's; $* * p<0.01 ; * * *<<0.001$. 


\section{b) ADDITIONAL MATERIAL FOR THE AP180 STUDY}

Appendix Table S14. Statistics for quantification of the subcellular distribution of AP180 after stimulation (related to Fig. 3-2B).

\begin{tabular}{|l|l|l|l|l|l|}
\hline Data & Wt rest & Wt stim & Statistical test & $\mathbf{t} / \mathbf{U}$ & p-value \\
\hline $\mathbf{3 - 2}_{\mathbf{i}}:$ : M embrane & $103 \pm 5.7$ & $107 \pm 4.8$ & Student's t-test & $\mathrm{t}=0.604$ & $\mathrm{p}=0.5468$ \\
\hline $\mathbf{3 - 2}_{\mathbf{i i}}:$ : Cytosol $(2 \mu \mathrm{m})$ & $53.3 \pm 3.6$ & $37 \pm 2.7$ & Student's $\mathrm{t}$-test & $\mathrm{t}=3.69$ & $\mathrm{p}=0.0003$ \\
\hline
\end{tabular}

Appendix Table S15. Statistics for Vglut3 and otoferlin fluorescence levels and for numbers of ribbon synapses in Wt and AP180-KO mice (related to Fig. 3-3).

\begin{tabular}{|l|l|l|l|l|l|}
\hline Data & Avg. $\mathbf{W t}$ & Avg. $\mathbf{K O}$ & Statistical test & $\mathbf{t} \mathbf{U}$ & $\mathbf{p}$-value \\
\hline $\mathbf{3 A}_{\mathrm{i}}$ : O toferlin & $100 \pm 4.6$ & $95.7 \pm 6.0$ & Student's t-test & $\mathrm{t}=1.37$ & $\mathrm{p}=0.17$ \\
\hline $\mathbf{3 B}_{\mathrm{i}}$ : V glut3 & $100 \pm 6.6$ & $82.5 \pm 6.1$ & Student's t-test & $\mathrm{t}=5.34$ & $\mathrm{p}<0.0001$ \\
\hline $\mathbf{3 C}_{\mathrm{i}}:$ N ribbon synapses & $14.5 \pm 0.4$ & $14.5 \pm 0.4$ & Student's t-test & $\mathrm{t}=0.054$ & $\mathrm{p}=0.96$ \\
\hline
\end{tabular}

Appendix Table S16. Statistics for quantifications of EM tomograms from ribbon synapses of Wt and AP180-KO mice (related to Fig. 3-4).

\begin{tabular}{|c|c|c|c|c|c|}
\hline Data & Avg. Wt & Avg. KO & Statistical test & $\mathbf{t} / \mathbf{U}$ & p-value \\
\hline 4E: N of RA-SVs & $47.7 \pm 1.7$ & $36.4 \pm 3.2$ & Student's t-test & $t=3.1$ & $p=0.0062$ \\
\hline 4F: N of MP-SV s & $18.5 \pm 1.5$ & $14.4 \pm 0.9$ & Student's t-test & $t=2.31$ & $p=0.0328$ \\
\hline $\begin{array}{l}\text { 4G: Distance to membrane } \\
\text { (MP-SVs) [nm] }\end{array}$ & - & - & $\begin{array}{l}\text { Kolmogorov- } \\
\text { Smirnov test }\end{array}$ & $D=0.239$ & $p=0.0002$ \\
\hline $\begin{array}{l}\text { 4G: Avg. }{ }^{*} \text { distance to } \\
\text { membrane(M P-SVs) }[n m]\end{array}$ & $20.1 \pm 0.7$ & $26.7 \pm 2.1$ & $\begin{array}{l}\text { M ann-W hitney U } \\
\text { test }\end{array}$ & $U=21$ & $p=0.0288$ \\
\hline $\mathbf{4 H}$ : $\mathrm{N}$ of coated structures & $8 \pm 0.7$ & $3.6 \pm 0.5$ & Student's t-test & $t=4.71$ & $p=0.0002$ \\
\hline $\mathbf{4 H}$ : N of coated vesicles & $6.9 \pm 0.8$ & $1.8 \pm 0.3$ & $\begin{array}{l}\text { M ann-W hitney U } \\
\text { test }\end{array}$ & $U=1$ & $p<0.0001$ \\
\hline 4H: $\mathrm{N}$ of coated pits & $0.4 \pm 0.2$ & $0.7 \pm 0.3$ & $\begin{array}{l}\text { M ann-W hitney U } \\
\text { test }\end{array}$ & $U=40.5$ & $p=0.4699$ \\
\hline $\begin{array}{l}\text { 4H: } N \text { of coated pits at } \\
\text { ELVs }\end{array}$ & $0.7 \pm 0.3$ & $1.1 \pm 0.2$ & $\begin{array}{l}\text { Mann-W hitney U } \\
\text { test }\end{array}$ & $U=34$ & $p=0.1626$ \\
\hline 4I: N of cytosolic SV s & $90.2 \pm 7.9$ & $37 \pm 4.6$ & Student's t-test & $t=5.82$ & $p<0.0001$ \\
\hline $\begin{array}{l}\text { 4J: Diameter of cytosolic } \\
\text { SV s [nm] }\end{array}$ & - & - & $\begin{array}{l}\text { Kolmogorov- } \\
\text { Smirnov test }\end{array}$ & $\mathrm{D}=0.44$ & $p<0.0001$ \\
\hline $\begin{array}{l}\text { 4J: Avg.\# diameter of } \\
\text { cytosolic SV s [nm] }\end{array}$ & $44.1 \pm 0.4$ & $48.1 \pm 0.6$ & Student's t-test & $t=5.56$ & $p<0.0001$ \\
\hline 4K: $N$ of ELVS & $21.3 \pm 2.3$ & $22.2 \pm 1.8$ & Student's t-test & $\mathrm{t}=0.304$ & $p=0.7645$ \\
\hline $\begin{array}{l}\text { 4L: Avg.\# ELV volume } \\
{\left[\mathrm{nm}^{3}\right]}\end{array}$ & $\begin{array}{ll}569,333 \quad \pm \\
28,301\end{array}$ & $\begin{array}{l}1,210,064 \pm \\
225,248\end{array}$ & $\begin{array}{l}\text { Mann-W hitney U } \\
\text { test }\end{array}$ & $U=1$ & $p=0.0043$ \\
\hline
\end{tabular}

\#. averages of each tomogram used for statistical analysis 
Appendix Table S17. Statistics for quantifications of EM tomograms from ribbon synapses of Wt and AP180-KO mice after stimulation (related to Fig. 3-5).

\begin{tabular}{|c|c|c|c|c|c|}
\hline Data & $\begin{array}{l}\text { Avg. Wt } \\
\text { stim }\end{array}$ & $\begin{array}{l}\text { Avg. KO } \\
\text { stim }\end{array}$ & Statistical test & $t / U$ & p-value \\
\hline 7D: $N$ of RA-SVs & $38.6 \pm 3.6$ & $34.1 \pm 1.6$ & $\begin{array}{l}\text { Mann-W hitney U } \\
\text { test }\end{array}$ & $U=39.5$ & $p=0.445$ \\
\hline 7E: N of M P-SVs & $16.1 \pm 1.9$ & $16.1 \pm 1.3$ & $\begin{array}{l}\text { Mann-W hitney U } \\
\text { test }\end{array}$ & $U=45$ & $p=0.7207$ \\
\hline $\begin{array}{l}\text { Avg. }{ }^{*} \text { distance } \quad \text { to } \\
\text { membrane(M P-SVs) }[\mathrm{nm}]\end{array}$ & $22.3 \pm 2.3$ & $24.4 \pm 1.4$ & Student's t-test & $t=0.77$ & $p=0.4505$ \\
\hline 7F: N of cytosolic SV s & $58.9 \pm 6.1$ & $34.4 \pm 2.8$ & $\begin{array}{l}\text { Mann-W hitney U } \\
\text { test }\end{array}$ & $U=12$ & $p=0.0026$ \\
\hline $\mathrm{N}$ of ELVs & $17.7 \pm 3.0$ & $24.0 \pm 2.5$ & Student's t-test & $t=1.6194$ & $p=0.1127$ \\
\hline $\begin{array}{l}\text { 7H: Avg.\# ELV volume } \\
{\left[\mathrm{nm}^{3}\right]}\end{array}$ & $\begin{array}{ll}770,287 & \pm \\
110,267 & \end{array}$ & $\begin{array}{l}1,418,517 \pm \\
288,188\end{array}$ & $\begin{array}{l}\text { M ann-W hitney U } \\
\text { test }\end{array}$ & $U=22$ & $p=0.0355$ \\
\hline 7I: N of coated structures & $5.3 \pm 0.4$ & $2.3 \pm 0.7$ & $\begin{array}{l}\text { Mann-W hitney U } \\
\text { test }\end{array}$ & $U=11.5$ & $p=0.0022$ \\
\hline 7I: $N$ of coated vesicles & $3.7 \pm 0.4$ & $1.6 \pm 0.5$ & $\begin{array}{l}\text { Mann-W hitney U } \\
\text { test }\end{array}$ & $U=12$ & $p=0.002$ \\
\hline 7I: $N$ of coated pits & $0.4 \pm 0.2$ & $0.3 \pm 0.2$ & $\begin{array}{l}\text { Mann-W hitney U } \\
\text { test }\end{array}$ & $U=45$ & $p=>0.999$ \\
\hline 7I: $N$ of coated pits at ELVs & $1.2 \pm 0.1$ & $0.5 \pm 0.2$ & $\begin{array}{l}\text { M ann-W hitney U } \\
\text { test }\end{array}$ & $U=32$ & $p=0.006$ \\
\hline
\end{tabular}

\#. averages of each tomogram used for statistical analysis 
Appendix Table S18. Statistics for tethering analysis in Wt and AP180-KO mice (related to Fig. 3-5G).

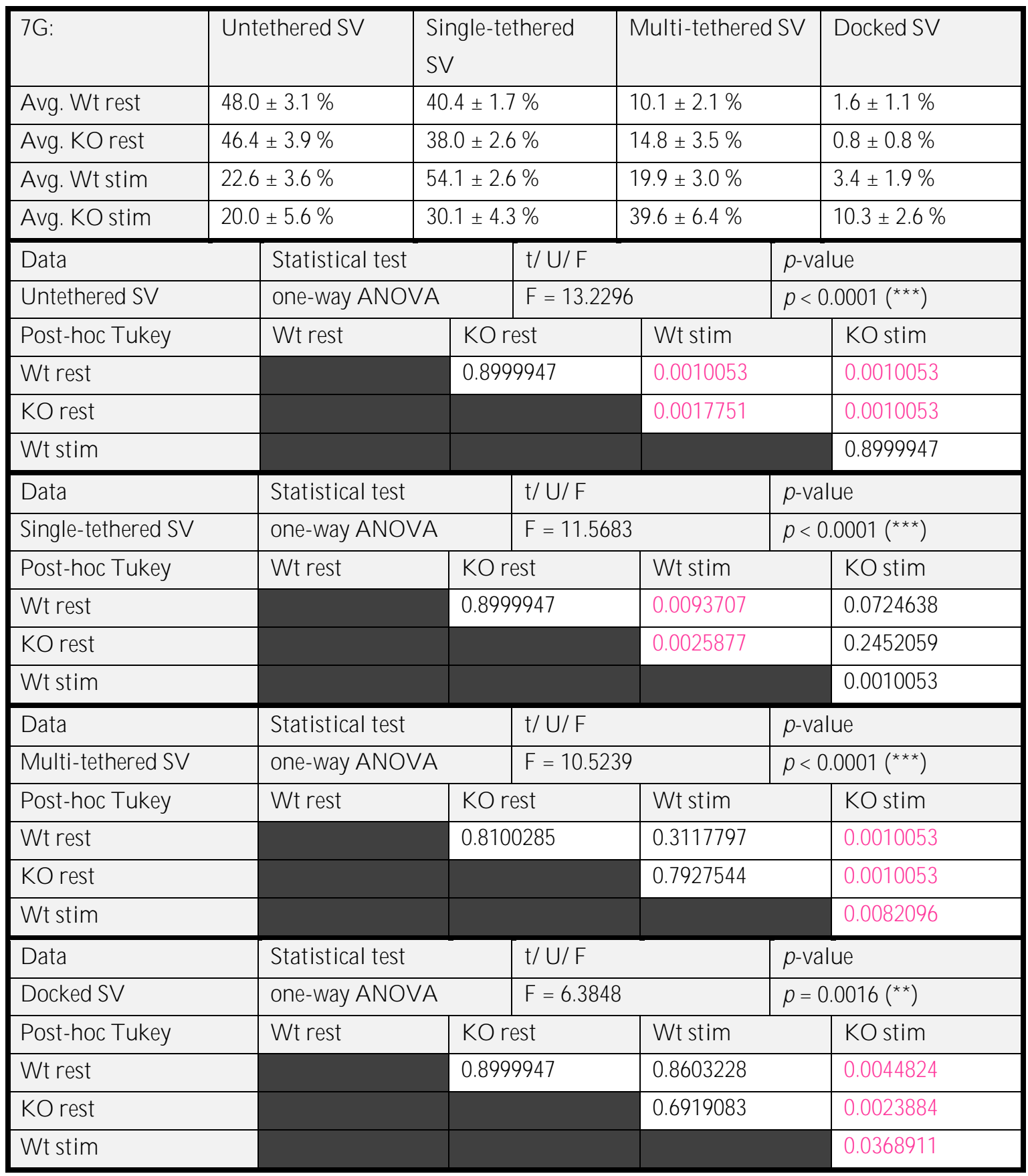




\section{ABBREVIATIONS}

\begin{tabular}{|c|c|c|c|}
\hline ADBE & Activity-dependent bulk endocytosis & LRRK2 & Leucine-rich repeat kinase 2 \\
\hline AMPA & $\begin{array}{l}\text { a-amino-3-hydroxy-5-methyl-4- } \\
\text { isoxazolepropionic acid receptor }\end{array}$ & MP-SV & M embrane-proximal synaptic vesicle \\
\hline ANTH & AP180 N-terminal homology & NSF & $\mathrm{N}$-ethylmal eimide-sensitive factor \\
\hline AP-2 & Adaptor protein 2 & $\mathrm{OHC}$ & Outer hair cell \\
\hline AP180 & Assembly protein 180 & $\mathrm{PI}(4,5) \mathrm{P}_{2}$ & Phosphatidylinositol 4,5-bisphosphate \\
\hline$A Z$ & Activezone & RA-SV & Ribbon-associated synaptic vesicle \\
\hline BAR & Bin-Amphiphysin-Rvs & RIM & Rab3-interacting molecule \\
\hline CALM & $\begin{array}{l}\text { Clathrin-assembly lymphoid myeloid } \\
\text { leukaemia protein }\end{array}$ & RIM-BP & RIM -binding protein \\
\hline CAZ & Cytomatrix of the active zone & RRP & Readily-releasable pool \\
\hline CCP & Clathrin-coated pit & SGN & Spiralganglion neuron \\
\hline $\mathrm{CCV}$ & Clathrin-coated vesicle & SH 3 & SRC H omology 3 \\
\hline CME & Clathrin-mediated endocytosis & SKO & Single-knockout \\
\hline CtBP2 & C-terminal-binding protein 2 & SNAP-25 & $\begin{array}{l}\text { Synaptosomal nerve-associated protein } \\
25\end{array}$ \\
\hline DKO & Double-knockout & SNARE & $\begin{array}{l}\text { SNAP (Soluble N SF Attachment } \\
\text { Protein) Receptor }\end{array}$ \\
\hline EEA 1 & Early endosomal antibody 1 & SRP & Slowly-releasable pool \\
\hline ELV & Endosome-like vacuole & SV & Synaptic vesicle \\
\hline EPSC & Excitatory postsynaptic current & TKO & Triple-knockout \\
\hline HPF & High-pressure freezing & VAMP & V esicle associated membrane protein \\
\hline IHC & Inner hair cell & Vglut3 & V esicular glutamate transporter 3 \\
\hline KO & Knockout & UFE & Ultrafast endocytosis \\
\hline Lamp-1 & $\begin{array}{l}\text { Lysosomal-associated } \\
\text { protein } 1\end{array}$ & Wt & W ildtype \\
\hline LDCV & Large dense core vesicle & & \\
\hline
\end{tabular}




\section{LIST OF FIGURES}

\begin{tabular}{|c|c|c|}
\hline FIGURE & TITLE & PAGE \\
\hline \multicolumn{3}{|c|}{ ○ INTRODUCTION } \\
\hline FIG. 1-1 & The anatomy of the mammalian inner ear. & 10 \\
\hline FIG. 1-2 & M orphological characteristics of IH Cs and ribbon synapses. & 13 \\
\hline FIG. 1-3 & Endocytic mechanisms at synapses. & 24 \\
\hline FIG. 1-4 & A bsence of endophilin-A results in disturbed SV recycling. & 31 \\
\hline FIG. 1-5 & AP180 is involved in neuronal SV reformation and release site clearance. & 34 \\
\hline \multicolumn{3}{|c|}{ O CHAPTER 1} \\
\hline FIG. 2-1 & Endophilin-A expression in the cochlea. & $46(3 / 22)$ \\
\hline FIG. 2-2 & $\begin{array}{l}\text { Reduced presynaptic } \mathrm{Ca}^{2+} \text { currents and exocytosis in endophilin-deficient } \\
\text { IHCs maintained in organotypic culture. }\end{array}$ & $47(4 / 22)$ \\
\hline FIG. 2-3 & Disruption of endophilins reduces $\mathrm{Ca}^{2+}$ influx of IHCs after hearing onset. & $48(5 / 22)$ \\
\hline FIG. 2-4 & Sustained exocytosis is impaired in endophilin-deficient IHCs. & $50(7 / 22)$ \\
\hline FIG. 2-5 & Disruption of endophilins causes a reduction of IHC otoferlin levels. & $51(8 / 22)$ \\
\hline FIG. 2-6 & Slowed endocytic membrane retrieval in endophilin-deficient IHCs. & $52(9 / 22)$ \\
\hline FIG. 2-7 & $\begin{array}{l}\text { Decreased number of small vesicles, accumulations of coated structures and } \\
\text { ELVs at IHC synapses of endophilin mutants. }\end{array}$ & $53(10 / 22)$ \\
\hline FIG. 2-8 & $\begin{array}{l}\text { Absence of endophilins causes changes in SV number and distribution at IH C } \\
\text { ribbon synapses. }\end{array}$ & $54(11 / 22)$ \\
\hline FIG. 2-9 & $\begin{array}{l}\text { High } \mathrm{K}^{+} \text {stimulation leads to accumulations of ELVs and an increased } \\
\text { occurrence of coated pits at ELVs in endophilin 1/2-DKOs. }\end{array}$ & $56(13 / 22)$ \\
\hline FIG. 2-10 & Putative functions of endophilins in IHCs. & $57(14 / 22)$ \\
\hline FIG. 2-EV 1 & $\begin{array}{l}\mathrm{Ca}^{2+} \text { inactivation remains unaffected in endophilindeficient IHCs after } \\
\text { hearing onset. }\end{array}$ & $66(1 / 3)$ \\
\hline FIG. 2-EV2 & Normal auditory brainstemresponses (ABRs) in $1-S K O$ and $1 / 3-D K O$ mice. & $66(1 / 3)$ \\
\hline FIG. 2-EV3 & M yosin 6 protein levels are unaltered in the absence of endophilins. & $67(2 / 3)$ \\
\hline FIG. 2-EV 4 & $\begin{array}{l}\text { M ethods to correct for the depolarization-independent decrease of } \mathrm{Cm} \text { in } \\
\text { endocytosis measurements. }\end{array}$ & $68(3 / 3)$ \\
\hline \multicolumn{3}{|c|}{$\circ \quad$ CHAPTER 2} \\
\hline FIG. 3-1 & AP180 is present in the murine organ of Corti. & 81 \\
\hline FIG. 3-2 & Subcellular distribution of A P180 changes after stimulation. & 83 \\
\hline FIG. 3-3 & Reduced V glut3 levels, but unaltered otoferlin levels in AP180-deficient IHCs. & 84 \\
\hline FIG. 3-4 & Ultrastructural changes of IHC ribbon synapses in AP180-KO mice. & 86 \\
\hline FIG. 3-5 & $\begin{array}{l}\text { SV -tethering to the AZ and SV reformation are altered in stimulated IHCS } \\
\text { from AP180-KO mice. }\end{array}$ & 89 \\
\hline \multicolumn{3}{|c|}{$\circ$ DISCUSSION } \\
\hline FIG. 4 & A putative model for synaptic transmission in IHCs. & 112 \\
\hline
\end{tabular}




\section{DECLARATION OF INDEPENDENT WORK}

This dissertation entitled "M orphological studies of synaptic vesicle recycling at the inner hair cell ribbon synapse" was written independently and with no other sources or aids than quoted.

Jana Kroll

Göttingen, December 2018 


\section{ACKNOWLEDGEMENTS}

During the past four years, I did not only get the chance to work in a stirring, motivating scientific environment - I also received a lot of support and made some precious friendships. I am grateful to all members of theSynaptic V esicle Dynamics Group, to the Institute for A uditory N euroscience and to the InnerEarLab. It has been a pleasure working with you.

I would like to especially thank the following people:

Ira M ilosevic: thank you for having me as a PhD student and for letting me work on this exciting project. Thank you for your support and for giving me the freedom to work so independently.

Tobias M oser: thank you for letting me work in your institute, for the fruitful cooperation on the endophilin project and for giving me the chance to work on the AP180 project. Beyond that, I would like to thank you for all your support and for proof-reading my thesis.

Carolin Wichmann: thank you for being my co-supervisor, for letting me work in your laboratory and for teaching me high-pressure freezing and electron tomography. Thank you for all your feedback, for all your advice and support. Thank you for always having an "open ear".

Nils Brose: thank you for being part of my thesis committee and for giving me helpful advice whenever needed.

Thomas Dresbach, Ellen Reisinger and Ralf Heinrich: thank you for being part of my examination board.

Jakob Neef: thank you for all your help and feedback, and for answering even the most stupid questions. Thank you for teaching me STED microscopy and Igor. And thank you for intensively proof-reading my PhD thesis.

Christian Vogl: thank you for always being honest and critical - all the feedback and discussions helped me a lot to also improve my own standards.

Lina Jaime: thank you for discussing the endophilin project with me again and again, thank you for all your feedback and for always motivating me.

Gerhard Hoch and Hanan AI-M oyed: thank you for helping me with Imaris/M atlab.

Andreia Cepeda: thank you for proof-reading my thesis. 
The BIN girls: thank you for the enjoyable working atmosphere, for interesting lunch conversations and for all your help. Ritu, thank you in particular for helping me with the freezing and the AFS.

Patricia Räke-Kügler, Susann M üller, Christiane Becker, SynnöveBeckh and the GGN B Office: thank you for the excellent administrative organization throughout my PhD.

I would like to thank all the other not yet mentioned collaborators of my PhD projects: Ilona Kondratiuk, thank you for providing the interaction studies. Nicola Strenzke: thank you for taking care of the systems physiology. Özge Demet Özçete: thank you for performing patch-clamp recordings for the AP180 project. Sangyong Jung: thank you for initiating the AP180 project. Tanja Maritzen: thank you for sending us the AP180 mice and for your editorial help with the AP180 manuscript.

I am extremely grateful to all the technicians who made my life in the lab so much easier. Anna Goldak, Christiane Senger-Freitag, Sandra Gerke, M elanie König and Dirk Schwitters: thank you for your excellent technical support and for all the genotyping. Anna: thank you especially for helping me with the HPM . Christiane: thank you for putting so much effort into the daily weight checks of the A P180 mice. N adine Dietrich and Stefan Thom: thank you for measuring A BRs.

Last but not least, I want to thank my family (yes, Markus, you are a part of my family) and my friends: Thank you for all your support, help and encouragement. 